\title{
Damage \\ in Laser Materials
}

U.S. ARTMENT OF MMMERCE National Bureau of Standards 

UNITED STA'TES DEPARTMENT OF COMMERCE • Maurice H. Stans, Secretary

NATIONAL BUREAU OF STANDARDS - Lewis M. Branscomb, Director

\section{Damage in Laser Materials}

Proceedings of a Symposium Sponsored by the American Society for Testing and Materials

and by the National Bureau of Standards

June 24-25, 1970, NBS, Boulder, Colorado

Edited by

Alexander J. Glass

Wayne State University

Detroit, Michigan 48202

and

Arthur H. Guenther

Air Force Weapons Laboratory

Kirtland AFB, New Mexico 87117

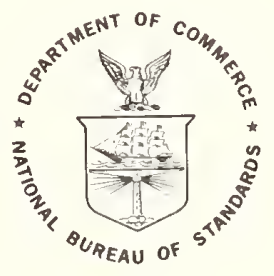

National Bureau of Standards Special Publication 341

Nat. Bur. Stand. (U.S.), Spec. Publ. 341, 126 pages (Dec. 1970)

CODEN: XNBSA

Issued December 1970

For sale by the Superintendent of Documents, U.S. Government Printing Office, Washington, D.C. 20402 (Order by SD Catalog No. C13.10:341), Price $\$ 1.25$. 


\section{Foreword}

These proceedings report in detail the formal papers and discussions presented at the 2 nd Annul Symposium on Damage in Laser Materials held at the National Bureau of Standards, Boulder, Colorado on June 24 and 25, 1970. This meeting was jointly sponsored by the National Bureau of Standards and the American Society of Testing and Materials. The major topics covered were Diagnostic Development, Damage Testing and Assessment, Damage Theory, and Physical Characteristics of Optical Media of importance in controlling damage.

The co-chairmen, Dr. Alexander J. Glass of Wayne State University, Detroit, Michigan, and Dr. Arthur H. Guenther of the Air Force Weapons Laboratory, Kirtland AFB, New Mexico, take full responsibility for synopsis of comments following each paper as well as the summary and conclusions of the meeting.

It is suggested that individuals interested in the subject obtain publication NMAB-271, "Report of the Committee on the Fundamentals of Damage in Laser Glass" which is available for sale îrom the National Technica?. Ir rortation Service, Springfield, Virginja 2215]. at a cost of $\$ 3.00$. Proceedings of the 1969 ASTM Symposium were published in December 1969 as "Damage in Laser Glass", ASTM Special Technical Publication No. 469 "Library of Congress Catalog Card No. 74-102757".

It is our intention to convene another symposium next year in Boulder during June to update and document the state of the art in Damage in Laser Materials at that time. This meeting will cover the subject historically presented at these symposia as well as attempt to attack new areas such as thin film coating damage, newer non-linear optical materials and crystalline laser materials (such as YAG, FAP, SOAP, in addition to ruby). Of particular importance will be discussions on damage thresholds in picosecond times. We wish to encourage the reader to contact us on matters pertinent to the intent of these conferences.

A. H. Guenther

Library of Congress Catalog Card Number: 73-609162 


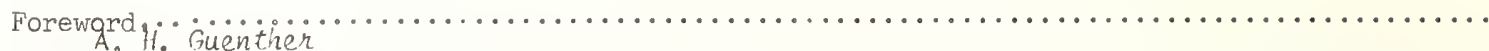

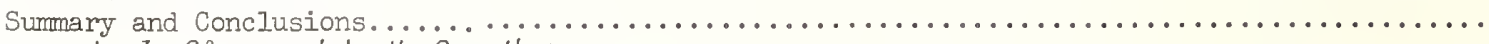

A. J. Glass and A. H. Suentizer

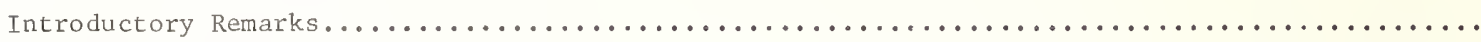
A. J. Glass

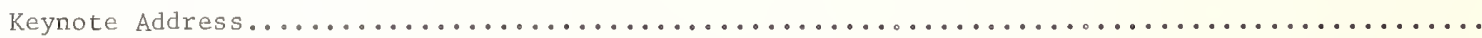
J. D. Myers

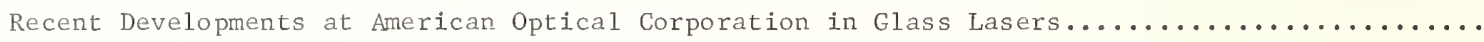
E. Snitzer

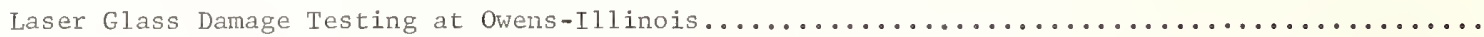
R. W. Beck

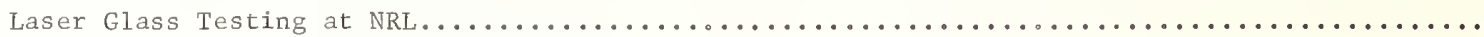
T. H. DeRieux and J. M. McMahon

Experimental Investigation of the Behavior of Neodymium-Glass Laser Amplifiers............ C. R. Jones, P. V. Avizonis, and P. Sivgals

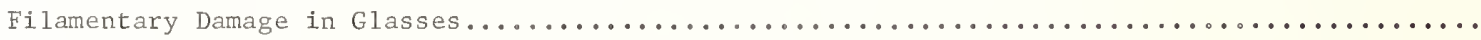
J. Davit

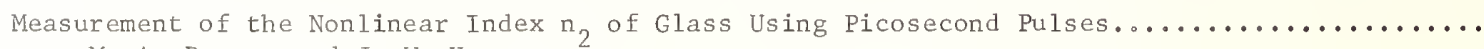
M. A. Duguay and $J$. W. Hansen

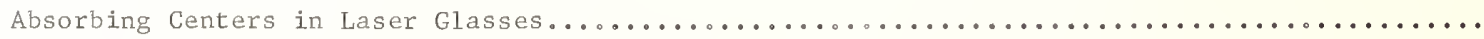
H. S. Bennett

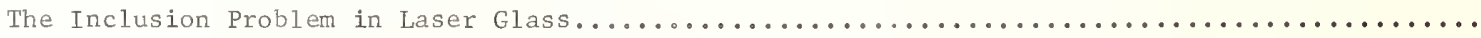
R. W. Hopper, C. Lee and D. R. Uhlmann

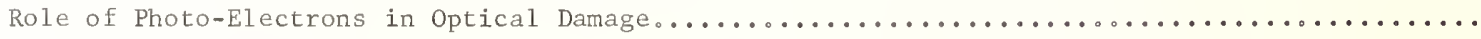
R. W. Hellwarth

Damage Threshold Studies in Ruby and Sapphire. C. R. Giuliano and L. D. Hess

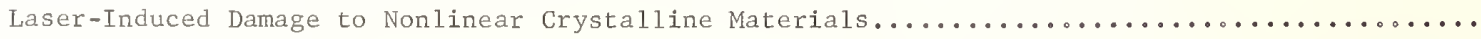
M. Bass

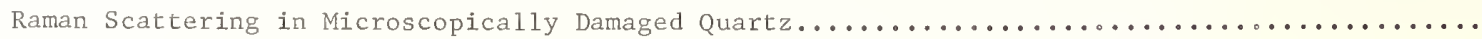
David F. Edwards, C. Y. She, J. D. Masso, Y. D. Harker and H. C. Schade

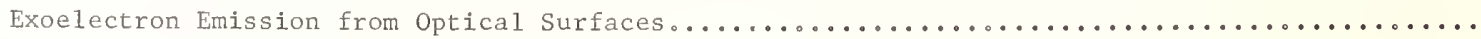
P. Bråunlich

Appendices

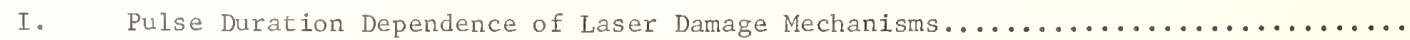
E. S. Bliss

II. Participants. 
The second ASTM Symposium on Damage in Laser Materials was held at the National Bureau of Standards at Boulder, Colorado on June 24th and 25th, 1970. About 150 attendees heard papers by fourteen speakers, representing the major manufacturers of laser glass, government, industrial and university laboratories. Although the primary emphasis was on Nd:glass as a laser material, damage mechanisms and morphology in ruby, sapphire, and nonlinear optic materials were also considered. It was clear that substantial advances have been made in the last year in the production of damageresistant laser glass for use in conventional Q-switched lasers. New diagnostic techniques have been developed, and in some cases, adopted as quality control procedures by the glass manufacturers. Theoretical understanding of the fundamental damage mechanisms is beginning to emerge, and material properties relevant to these damage mechanisms are beginning to be measured. It was equally clear that a great deal of effort remains to be expended to develop techniques suitable for nondestructive testing of laser materials, and that many properties of laser materials at high energy density are presently poorly understood.

Key words: Ferroelectric materials; glass; laser damage; lasers.

\section{Summary and Conclusions \\ 1. Principal Conclusions}

In the course of the two days of discussion, certain points emerged which might be termed "highlights" of the meeting. It was dramatically clear that both major domestic manufacturers of laser glass have improved the damage resistance of their product significantly in the past few years. Laser glass is presently commercially available from both the American Optical Company and 0wens-Illinois which will withstand from 20 to 30 joules $/ \mathrm{cm}^{2}$ in a $30 \mathrm{nsec}$ pulse. Selected samples are capable of operating at higher flux densities. Facilities now exist at these companies, and at the Naval Research Laboratories, for testing large sized samples of laser glass at energy densities of 40 to 50 joules $/ \mathrm{cm}^{2}$, unfocused, in 30 to $50 \mathrm{nsec}$ pulses. In addition, new quality control procedures have instituted by the manufacturers to select glass of better homogeneity, free of striae, bubbles, stones, and other imperfections.

A point which was continually emphasized throughout the symposium was the importance of carrying out damage tests with mode-controlled lasers. In both ruby and nonlinear materials, damage thresholds under illumination by single-mode lasers were from 10 to 100 times greater than those reported under multimode illumination where average energy densities are computed. The scatter in the values obtained for damage thresholds was also greatly reduced by using well controlled and reproducible lasers thus eliminating scatter due to flucuations in the irradiating source.

Two speakers reported theoretical analyses of the stress induced in an otherwise homogeneous medium by the presence of an absorbing inclusion under intense illumination. Although there were points of disagreement between the two analyses, certain conclusions were obtained from both. The most damaging particles range in size from $10^{-5}$ to $10^{-4} \mathrm{~cm}$, and it is precisely in this range that the non destructive detection of particulate inclusions is most difficult. Larger particles do not get heated to a temperature sufficiently high to cause damaging stress concentrations, while smaller particles do not intercept enough of the incident energy to cause damage. Increasing the thermal conductivity of the medium increases the damage threshold, as might be expected. It is significant that detailed modeling of this most fundamental damage process has only been carried out recently, although the role of particulate inclusions in the damage process has been known for many years. The models, though approximate, show trends and parametric dependence which are useful to guide experimental research and device development.

An apparently new phenomenon which was reported at this symposium was the presence of a so-called "gain anomaly" in high energy, glass amplifier systems. At high pump energies, the gain measured in power amplifiers was up to $20 \%$ less than that expected from theory. This anomalous gain reduction could not be attributed to increased superradiant losses, spectral shift of the flashlamps, or the existence of any broad, pump-induced absorption in the laser medium. It appears in all laser glasses.

New diagnostic techniques were proposed for nondestructive tests of laser materials, including holographic identification of large inclusions, changes in the Raman spectrum as evidence of fatigue or residual stresses, and exoelectron emission as a tool for studying surface states. All these techniques are currently under further investigation. 


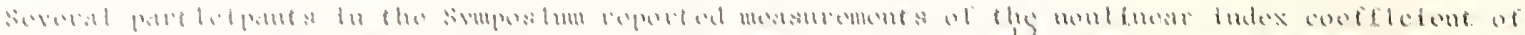

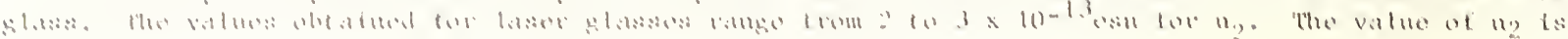
แ11

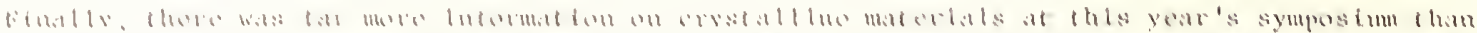

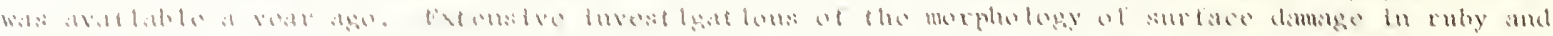

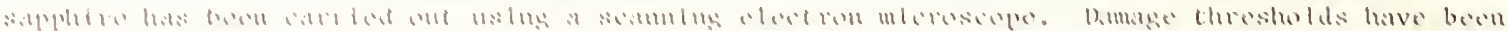

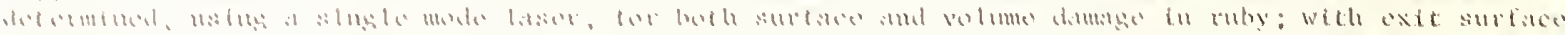

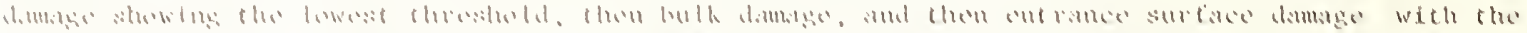

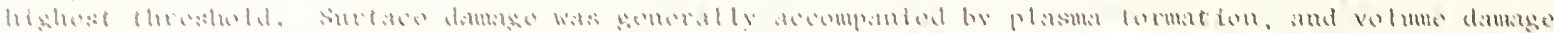

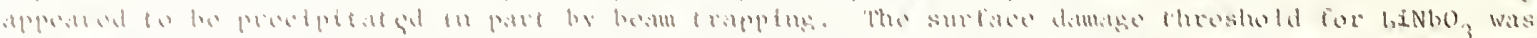

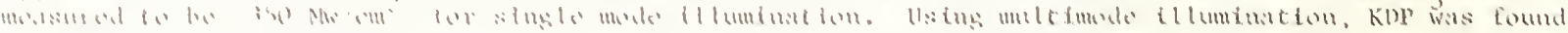

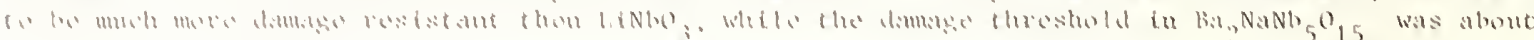

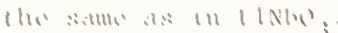

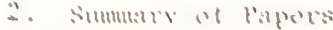

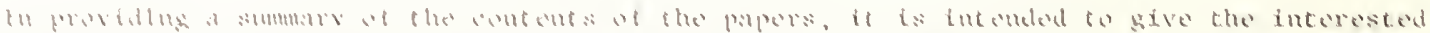

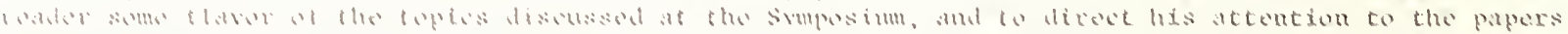

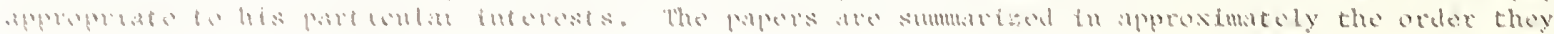

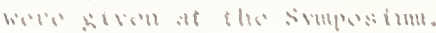

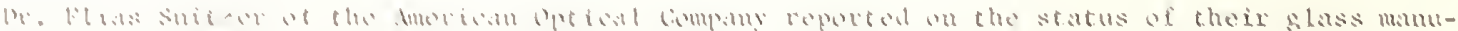

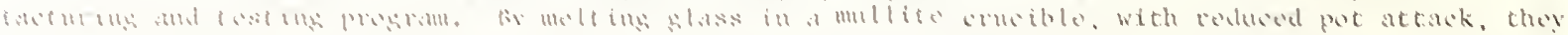

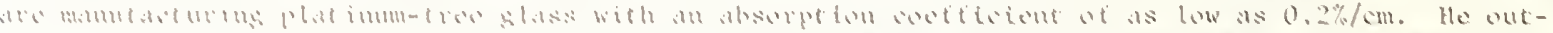

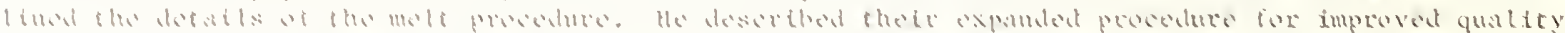

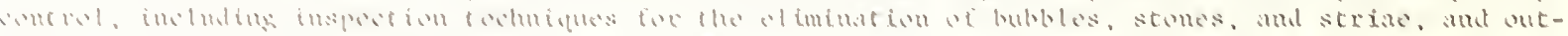

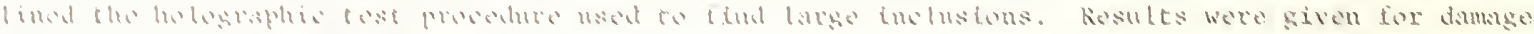
10:2

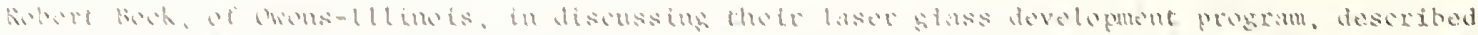

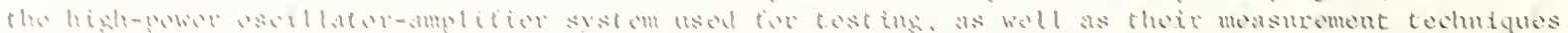

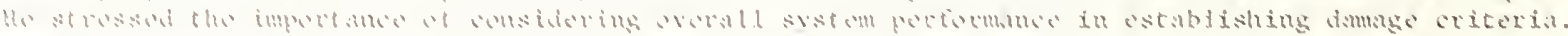

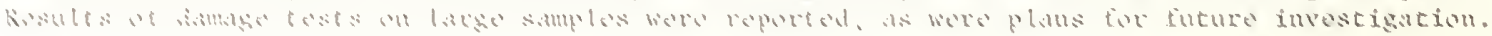

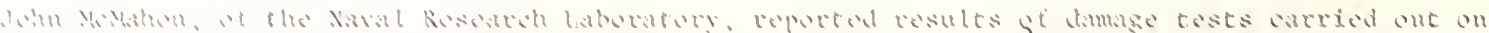

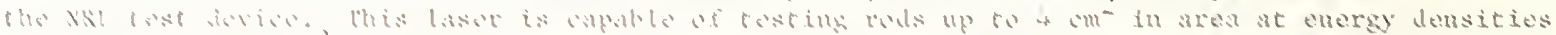

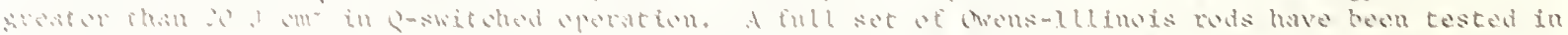

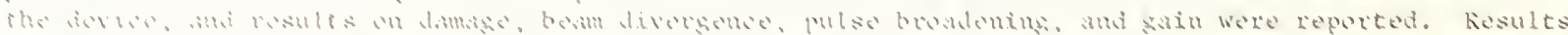
w口:

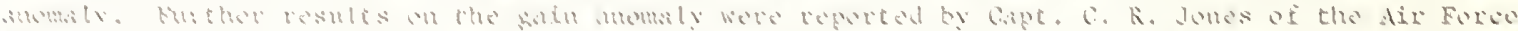

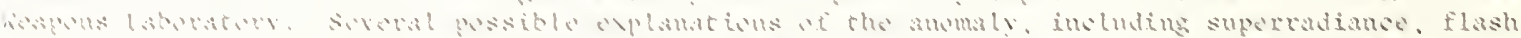

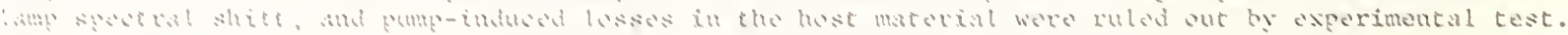
Aيst הin!

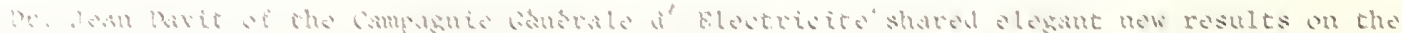

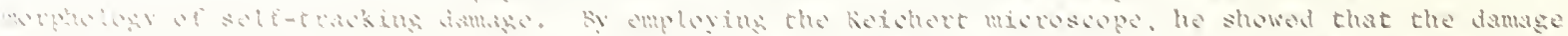

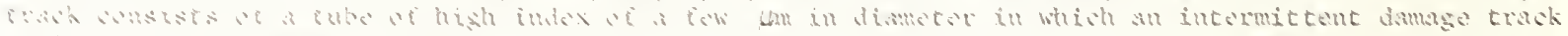

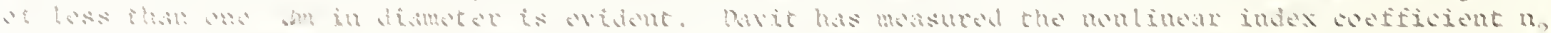

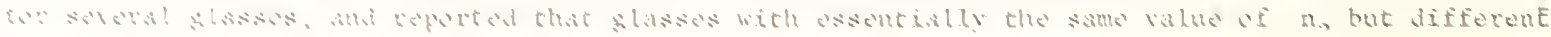

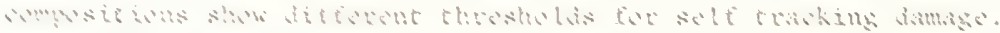

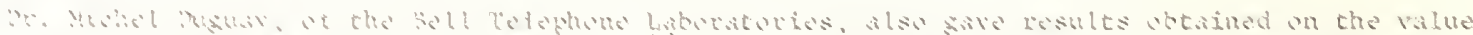

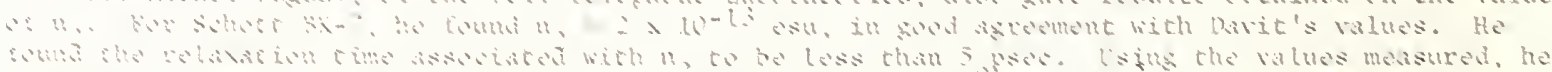

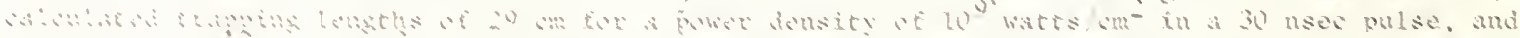

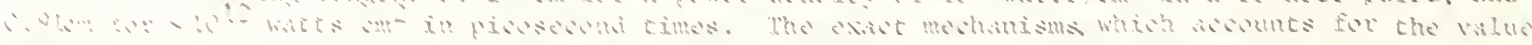

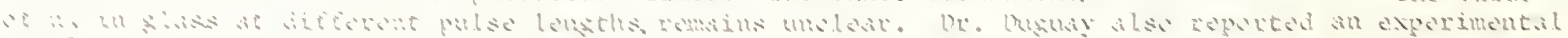

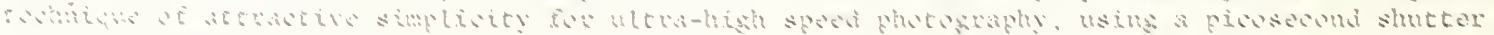

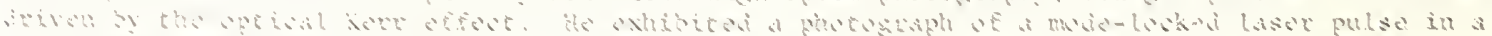

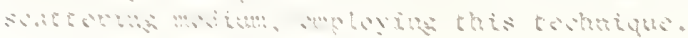

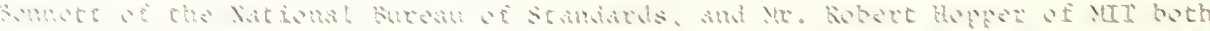

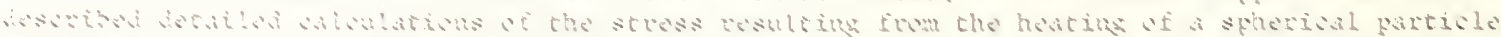

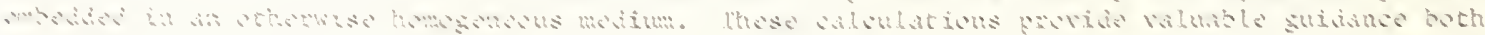

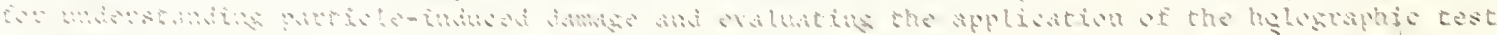

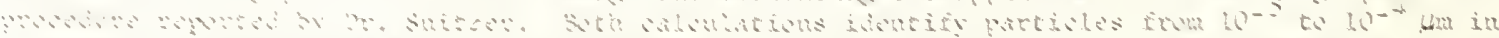

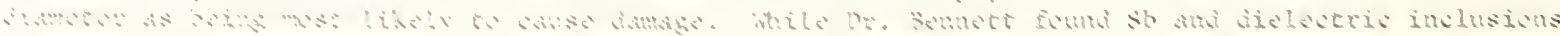
ㄴ. 
dielectrics. Dr. Bennett concluded that the damage process was more sensitive to the thermal conductivity of the host medium, compared to the thermal expansivity. He also concluded that "strengthening" the host by increasing its Young's modulus did not in itself ensure greater resistance to damage. Mr. Hopper concluded that great care had to be exercised in extrapolating damage results obtained with $\mu$ sec pulses to the nsec pulse regime. He also commented on the effect of non-sphericity of the particles on the computed damage threshold.

Dr. Robert Hellwarth of the Hughes Research Laboratory and the University of Southern California discussed a theoretical investigation of the heating of electrons by intense 1ight pulses, in polar crystals. Using the Fröhlich-Hamiltonian to describe the electron-phonon interaction, he concluded that there would not be significant production of "hot" electrons, but that the electrons would rapidly transfer the absorbed energy to the phonons. He estimated that the intensity of the resultant acoustical waves could be sufficient to cause damage. He also found that the presence of cold electrons could greatly enhance the excitation of impurity levels, and that an added multi-photon absorbtion by impurity centers could result. These results were in disagreement with those previously reported in the literature. Dr. Hellwarth also commented on the influence of conduction electrons on selftrapping, and identified the lack of experimental data on the photoconductivity of ruby and sapphire over an extensive range of light intensities as a serious deficiency.

Dr. Concetto Guiliano, of the Hughes Research Laboratory, showed scanning electron microscope pictures of surface damage in ruby samples. He found evidence of exit surface damage, accompanied by plasma formation, at a lower threshold than that observed for volume damage, which could be attributed to self-trapping. At still higher power levels, entrance surface damage, with plasma formation, was observed. Electron micrographs showed evidence of both crazing of the surface and of melting after repeated exposures. Dr. Guiliano conducted his tests with a mode-controlled ruby laser, which he described in detail. He also reported preliminary results for damage in optically pumped samples. There was no discernable difference in the damage threshold under these two conditions or when the samples were chemically treated. There was, however, a marked difference in the morphology of damage as exhibited in ruby and sapphire. Finally, in direct opposition to the findings of Nath and Walda, the addition of the suggested amounts of $\mathrm{TiO}_{2}$ to ruby did not improve the damage threshold and in fact, a decrease was observed.

Dr. Michael Bass, of Raytheon, discussed the question of damage testing in nonlinear optical materials. He emphasized the importance of detailed characterization of the material tested, and of careful control and measurement of the intensity profile of the irradiating laser. Evidence of the importance of laser mode-control was given by the fact that in samples of Lithium Niobate which were tested, the threshold for surface damage was 350 Megawatts $/ \mathrm{cm}^{2}$ in a single mode beam, but only 6 Megawatts $/ \mathrm{cm}^{2}$ in a multi-mode beam. These figures refer to average power density at the focus. In these experiments, surface damage was detected by the presence of a visible spark, and the subsequent microscopic detection of a damage pit on the surface. Other materials investigated include KDP, ADP, and Barium Sodium Niobate. No dependence of damage threshold on temperature or on the atmosphere to which the sample was exposed was observed. Dr. Bass pointed out that in most Q-Switched lasers or frequency doubled systems, the nonlinear crystal is the Achilles heel of the system, and that damage considerations often force one to the use of $\mathrm{KDP}$, which has a higher damage threshold, in place of the niobates, which exhibit greater nonlinear coefficients.

Three short papers concluded the symposium. Dr. David Edwards, of Colorado State University, discussed the use of Raman scattering as a tool for detecting local stress in crystalline materials. Stress accumulation is a precursor to catastrophic damage. In crystalline quartz, he has seen line broadening, line shifts, intensity changes, and even the occurence of new lines in stressed samples. Observations on laser-damaged quartz correlate with those made on samples subjected to thermal and uniaxial stress.

Dr. Peter Bräunlich, of the Bendix Company, reported on the use of exoelectron emission as a diagnostic tool for the study of surface states. By recording the emission of electrons from a surface as a sample is heated from low temperature, information can be obtained on the presence of electrons in shallow traps and defects near the surface. Using a Channeltron imaging device, a spatial image can be made of the variation of the work function on the surface. Results were reported for ED-2 glass.

Capt. Erlan Bliss, of the Air Force Cambridge Research Laboratory, discussed the influence of the various mechanisms of nonlinear index change on laser damage, as a function of the time duration of the laser pulse. An expanded version of his comments is included as an appendix to the symposium proceedings. 


\section{Recommendations for further work}

It is appropriate to end this summary of the conference with some observations on those features of damage in laser media not fully resolved, in which more examination is suggested. Obviously additional effort is necessary in the development of nondestructive diagnostic techniques for quality control purposes. It was pointed out on numerous occasions that as yet there is no convenient method to determine the presence of sub-micron sized inclusions in laser glass, which are the lowest threshold sites for the onset of inclusion damage under short pulse (i.e. $<30$ ns) conditions.

In regard to bulk damage, due to nonlinear effects such as self focussing, no clear evidence has been advanced which delineate the temporal domain over which a certain mechanism (e.g. A. C. Kerr Effect, electrostriction, etc.) is dominant as to its influence on the nonlinear refractive index n, Only the generally accepted thesis as to which is obviously the principal cause at either very short or very long times, leaving unclear the mechanism effective for many materials in the time regime in which most Q spoiled high power lasers operate.

With the rapid growth of applications for mode locked pico-second lasers, damage thresholds at these short times should be assessed to determine how the damage mechanism is modified by enhanced nonlinear optical behavior. This information is most crucial to the scientist interested in plasmas produced by lasers.

To people interested in high total energy, high peak power application, the new well documented evidence of a decrease in extraction efficiency under hard pumping conditions is most disconcerting. One must elucidate the cause of this anomalous behavior before one can determine whether he has to live with it or can, in some manner, correct it.

Finally, while it was reported that much higher damage thresholds are obtained when employing well-controlled single mode systems as compared to multimode lasers, the full danger of extrapolation of small area or small volume damage investigations to usable dimensions has not yet been conclusively demonstrated. This is particularly important in crystalline media which are less reproducible in the growth process than glass melting and fabrication.

A. J. Glass

A. H. Guenther 
Introductory Remarks

\author{
Alexander J. Glass \\ Wayne State University \\ Detroit, Mich. 48202
}

Welcome to the 2nd ASTM Symposium on Damage in Laser Materials. The ASTM Subcommittee II on Iasers and Laser Materials is charged with the responsibility of formulating standards for laser materials, components, and devices. It is essential that these standards be realistic, realizable in the laboratory, and wherever possible, be based on a full understanding of the fundamental processes involved in device performance and material properties.

In high power lasers, the damage level under various operating conditions is the material property of paramount interest. But before damage standards can be enunciated, substantial advances are required in the understanding of damage phenomena, and in the techniques of testing laser materials at high power. It is the concern of this symposium to inform the participants of the latest developments in laser materials and test procedures, and to reach a consensus on the state of knowledge regarding damage phenomena.

During the past few years, the level of understanding of the damage mechanisms in glass lasers, operating in a Q-switched pulse of 10 to $50 \mathrm{nsec}$ duration, has greatly increased. Three principal mechanisms of damage have been identified, damage to particular inclusions, damage to surfaces, and failure of the material in bulk due to the intense electric fields generated in self-trapped optical filaments. The principal source of particulate inclusions has been identified as platinum coming into the melt in the form of platinum oxide. A few different manufacturing techniques have been developed to circumvent this problem, either by preventing the formation of damaging inclusions of platinum, or eliminating platinum from the manufacturing process altogether.

As evidence of the success of these techniques, laser glass is now commercially available capable of withstanding light fluxes in excess of $20 \mathrm{~J} / \mathrm{cm}^{2}$ in $30 \mathrm{nsec}$ pulses, which represents an increase in damage level of a factor of 10 over the laser glass of a few years ago.

There have also been significant advances in the understanding of surface damage. There is general agreement that surface damage arises from the formation of a micro plasma at or within the surface. A somewhat empirical approach to treating the surface, to inhibit this plasma production, has proved successful in raising surface damage thresholds in excess of $100 \mathrm{~J} / \mathrm{cm}^{2}$ in $30 \mathrm{nsec}$ pulses.

The phenomenon of self-trapping is inherently complex, depending on the nonlinear index coefficient of the glass, the beam profile, and the length of path available. The underlying mechanism is a combination of effects, including electronic polarizability, molecular libration, Kerr effect and electrostriction. Basic measurements of the effective non-linear index as a function of pulse length are just emerging, and will be reported at this meeting.

There still remains a great deal of work to do on damage mechanisms. There is a scarcity of data at pulse lengths on one nanosecond or less. The question of surface damage as a function of surface condition and treatment is still somewhat open. There is still hope of further improving the damage level in glasses by 
reducing the size and frequency of occurence of inclusions. Test procedures must be developed if uniform standards are ever to emerge. When we turn our attention away from glass to crystalline materials, less is available in the way of systematic data. These materials, ruby, sapphire, and the materials of non-linear optics, are less homogeneous than glass, and reproducibility and materials specification are important problems. We shall hear two papers on damage in crystalline materials at this meeting.

The idea of this symposium is to bring us up to date on the subjects discussed. I want to thank all the speakers who have contributed papers, and all the attendees who will contribute to the discussion. We are grateful to the National Bureau of Standards for their hospitality. Our particular thanks go to Mrs. Pauline Smith and Dr. Harold Boyne of the National Bureau of Standards for their help and advice in the organization of this symposium, and their efforts in the preparation of the symposium proceedings. 
The American Society for Testing and Materials is sponsoring another Laser Damage Symposium for a number of reasons, most of which are intimately connected with the interests of the membership.

The primary reason, however, is simply in response to the rhetorical question, "How can we prepare standard methods for measuring the damage threshold if we are not really sure of what we are talking about?" On the other hand, one might well ask, "Why do anything at all?"

The situation which motivates most of us to action is, in this case, typical of new technologies. This situation can best be described as one of incomplete communication. What usually transpires is that some company makes a claim about one of its products. Other companies make counter-claims based upon their particular bent, until the air is filled with claim, counter-claim, and countercounter-claim...all using the same words, but within entirely different context. We all remember when the output of a laser was not so much dependent upon the electronics involved as whose calorimeter was used.

To elaborate further - one can imagine the following one-sided telephone conversation between a laser material user and the Ersatz Company who has just made a claim that its laser material has a damage threshold of .3 BTU/in ${ }^{2}$...

"Hello, Ersatz Company, I want to talk to one of your technical people about your laser material. Dr. Schwartz? That, will be fine. Hello, Bernie, how are you? What is this I hear about indestructible laser material? How did you run the test? Uh-huh...long focal length lens. What was the pulse width?...127 nanodays? How did you manage that? I see, it just came out that way. What would be an equivalent damage threshold at 2 picoseconds? You haven't the faintest idea? Well, in any case, it must be pretty good stuff...I imagine you tested quite a few samples. How big was the sample...microscope slide? Well, this is the same material you sell isn't it....almost? Can I buy some to try in my system? Problems...? What kind of problems? Man who made the stuff quit... to start a chicken farm?"

And, so it goes...

I am sure everyone here is familiar with similar episodes which have resulted in warped perspectives and agonizing frustration for all concerned. It seems clear that what is required to alleviate these misunderstandings is simply a clear presentation of the purpose for which the claim. is made, along with the context within which the claim applies. And, this is precisely what AstM attempts to accomplish. True, we do add a bit. We not only explain the purpose and scope, but also suggest a pre-tested measurement method.

So far, our efforts in this area have resulted in the following specifications which are now undergoing review and preparation for inclusion in the ASTM standards.

1) "MEASURIng the OUtPut OF A HE-NE LASER OPERAting AT $6328 \AA . "$

2) "INSPECTION PROCEDURE FOR SURFACE FINISH OF POLISHED CRYSTAL OPTICAL ELEMENTS."

3) "METHOD OF TEST OF BEAM DiVERGENCE FOR OPTICALly PUMPED PULSED LASERS."

4) "DETERMINATION OF PARALLELISM OF SURFACES OF TRANSPARENT MATERIALS."

5) "METHOD OF TEST FOR EFFECTIVE FLUORSCENT LIFETIME OF NEODYMIUM AND ERBIUM LASER MATER IALS."

6) "METHOD OF TEST OF SLOPE EFFICIENCY AND LASING THRESHOLD FOR RUBY LASER RODS."

7) "METHOD OF TEST OF LASING THRESHOLd CURRENT AND SLOP EFFICIENCIES FOR DISC LASERS."

Plus more to come... 
These standards have been prepared by the various sections of the subcommittee. The sections are: Optically Pumped Section, chaired by Arthur H. Guenther, U. S. Air Force Weapons Laboratory; the Electron Impact Section, chaired by Sheldon Minkowitz, The Perkin-E1mer Corporation, the Diode Section, co-chaired by Jack Ludman, Air Force Cambridge Research Laboratory and Robert J. Mahler, National Bureau of Standards, Boulder; and the Modulator Section, chaired by Allan E. Carlson, Gould, Inc.

We approach these subjects in a rather pragmatic manner. For example, if two solutions to a problem exist, rather than choose between them we suggest them both. Our mission is to foster understanding within and promote the well being of the laser industry as a whole, rather than restrict or regulate. All of us are volunteers, (although I suppose some are drafted) who feel the job needs doing and really can't find anyone else to do it.

WELCOME, on behalf of ASTM, to the second Laser Damage Symposium. 
Recent Developments

at American optical Corporation

in Glass Lasers

E. Snitzer 1

Central Research Laboratory

American Optical Corporation

Southbridge, Massachusetts 01550

A description will be given of the quality control procedures used to obtain laser glass from an all ceramic melt facility which is suitable for use at $20 \mathrm{~J} / \mathrm{cm}^{2}$ in a $30 \mathrm{~ns}$ pulse. A non-destructive holographic method for the identification of damage sites will be presented. Finally, some results will be given on recently prepared experimental glass which appears to be entirely free of damaging inclusions and which has an absorption coefficient at $1 \mu$ of $0.2 \% / \mathrm{cm}$.

Key Words: Glass damage, glass lasers, holography, inclusions, lasers.

\section{Introduction}

A review of the work done on laser induced damage in glass at American Optical corporation up to last year was given at the First conference on Damage in Laser Glass held here in Boulder a year ago [1]. Since that time, our efforts have been directed primarily towards the problem of damaging inclusions in neodymium laser glass. In this report, the quality control procedures that are used for obtaining glass suitable for operation up to $20 \mathrm{~J} / \mathrm{cm}^{2}$ in a $30 \mathrm{~ns}$ pulse without damage are described. Since damaging inclusions within glass are essentially a statistical problem, some data is given on the density of damage sites as a function of incident energy density. The second topic to be covered is some preliminary results on a nondestructive method for identifying potentially damaging inclusions by the use of holographic interferometry. Finally, some results on recently melted experimental glasses are given in which there appear to be no damaging inclusions.

\section{Quality Control and Damage Test Results}

Laser glass suitable for efficient operation in large oscillator-amplifier systems should not only be free of damaging inclusions but should have a low loss coefficient at $1.06 \mu$ and be of sufficient $1_{Y}$ good optical quality to permit output beams approaching diffraction limited performance. To obtain a low loss coefficient, the batch from which the glass is reacted contained less than $5 \mathrm{ppm}$ of iron and copper. The ceramic melter was made of high purity mulite, which is sufficiently insoluble in the glass so that the pot attack is low enough to permit effective homogenization of the glass. The glass was produced by a batch process in a mullite crucible with a bottom orifice [2]. Eight liters of glass are reacted, fined and homogenized in a ten liter crucible. Typically only five or six liters of finished glass is extruded at one time. The 
remaining glass in the crucible serves to plug the bottom orifice for the next fill.

The billets are cast in a graphite mold $7.5 \mathrm{~cm}$ in diameter and approximately a meter in length. After the ends are ground and polished for preinspection and the billet found to be reasonably free of bubbles and striae, the billets are placed in a transparent rectangular chamber filled with index matching immersion oil. A photographic record of the striae in the glass is then taken in the form of three shadowgraphs, one through the length of the billet and the other two photographic records perpendicular to the axis of the billet. The preinspection yields 60 to $80 \%$ of the billets produced. An average of 10 billets can be made with one crucible. Of these, 60 to $80 \%$ are sufficiently free of striae and bubbles to warrant further inspection in the liquid immersion tank. The glass coming from the tank typically contains no more than one bubble per two liters of glass with the bubble size less than $0.5 \mathrm{~mm}$ in diameter. The density of stones is of the order of one per ten liters. The yield from both the preinspection and the striaegraph test in the liquid immersion tank is between 30 and $40 \%$. The higher yields are for low neodymium content glass, that is, glass with either 0.8 or $1.5 \mathrm{wt} \% \mathrm{Nd}_{2} \mathrm{O}_{3}$. The specification on the loss coefficient at the laser wavelength of $1.06 \mu$ is that it not exceed $0.35 \% / \mathrm{cm}$; however, most of the glass has a loss coefficient of about $0.2 \% / \mathrm{cm}$. The glass of good optical quality is then damage tested.

The damage tester was described in detail by Young and Woodcock [1]. By use of pulse forming networks and a Kerr cell, the peak portion of the spontaneous emission from a meter length rod can be admitted to the subsequent amplifying stages with a total gain of $150 \mathrm{~dB}$. A schematic representation of the device is shown in Fig. 1. Three different pulse durations have been worked with as shown in the table in Fig. 1. Most of the damage testing was done with an energy per pulse of between 80 and 100 joules with a total pulse duration of $10 \mu \mathrm{s}$. Due to gain saturation, one half of the energy is contained in the first $4 \mu \mathrm{s}$. Gain saturation in the amplifier does not occur for the shorter pulse durations. Less energy was produced per pulse in the shorter times as shown in the table. The output diameter of the last amplifier was $38 \mathrm{~mm}$ and the full beam width at one half intensity was 1.8 milliradians. To obtain uniformity across the aperture only the central $30 \mathrm{~mm}$ was focused into the billet to be tested. The lens for focusing the energy into the billet depended on the energy generated in the pulse. For the three time domains, focal lengths of 4, 1.3 and 0.5 meters were used to test the glass. The most extensive tests were carried out with the $10 \mu \mathrm{s}$ pulse. A 4 meter focal length lens has sufficient depth of focus to sweep the entire length of the billet in one shot.

The test results on ten billets with $80 \mathrm{~J} / \mathrm{cm}^{2}$ in $10 \mu \mathrm{s}$ gave between two and six damage sites per billet. The sites appeared to be randomly distributed. The average density of damaging inclusions was one per liter. The glass which survived this test was then subjected to the next shorter pulse time. The density of damage sites as a function of energy density in the pulse is shown in Fig. 2. Curves A and B are for two different glasses and represent the typical spread in the average density of damage sites for various glasses. Glass which survived $80 \mathrm{~J} / \mathrm{cm}^{2}$ in $10 \mathrm{~ns}$ did not damage at $30 \mathrm{~J} / \mathrm{cm}$ for pulses between 0.5 and $1 \mu \mathrm{s}$ in duration. However, for energy densities above $30 \mathrm{~J} / \mathrm{cm}^{2}$ in this shorter time domain, the density of damage sites rose abruptly. The glass was then tested at $25 \mathrm{~J} / \mathrm{cm}^{2}$ in a $100 \mathrm{~ns}$ pulse and no damage was produced. Finally, tests at $20 \mathrm{~J} / \mathrm{cm}^{2}$ in $30 \mathrm{~ns}$ produced no additional damage sites. The damage sites which were produced in any of these time domains grew larger on subsequent irradiation at the same energy density, but no new damage sites appeared, which indicated that fatigue effects were not present.

A density of one damage site per liter is sufficiently low to readily permit fabrication of rods $30 \mathrm{~cm}$ or more in length, but a higher density of damage sites restricts the rod sizes that could be obtained. Hence, a practical restriction on the laser glass commercially available from $A O$ at the present time is that it be used in systems operated up to $20 \mathrm{~J} / \mathrm{cm}^{2}$ in a $30 \mathrm{~ns}$ pulse. At higher energy densities in this time domain, the glass may damage.

The damage produced as a function of pulse duration and energy density can be used to infer the approximate particle sizes for inclusions. In the paper to be 
presented at this conference by Hopper and Uhlmann, they calculate the temperature of a metallic inclusion as a function of energy density, pulse duration and particle size. From the temperature distribution, they obtain the stresses in the glass which can lead to fracture. Their analysis indicates that the damaging particles are about $1 \mu s$ in radius.

\section{Holographic Testing}

Although recently, some experimental melts of glass have been prepared which are entirely free of inclusions, the ability to identify the composition of the inclusion would be of great help for the production of large volumes of damage free glass. At present, by testing the glass with $80 \mathrm{~J} / \mathrm{cm}^{2}$ in $10 \mu \mathrm{s}$ when a damage site is identified by fracture of the glass, it is not possible to then determine the composition of the inclusion. This is because when damage occurs, a large surface area is presented over which the material in the inclusion is spread. Attempts have been made on damaged glass both with an electron beam probe and a laser spectroscopic microprobe to determine the composition of the material at the damage site. These attempts have been unsuccessful, probably because of the aforementioned problem of dispersal of the inclusion over a much larger volume.

By a holographic interferometric technique, it should be possible to identify the positions of the damaging inclusions within the glass without producing damage. Even though the particle size is of the order of $1 \mu \mathrm{s}$, if enough energy is absorbed from an incident beam of relatively long pulse duration, the heated glass in the vicinity of the particle could constitute a sufficiently large phase object to be seen optically. With care it is possible to see phase objects of less than $\lambda / 10$ [3]. Energy densities of thousands of joules per $\mathrm{cm}^{2}$ in several milliseconds may be required.

In the holographic interferometric technique, two holograms are superposed on the same film. The first exposure is taken just before irradiation with a high energy density and the other shortly after irradiation. In the reconstruction of the hologram, the fringe pattern in the vicinity of the particle would indicate the presence of an absorbing inclusion. An inclusion which is nondamaging, such as a small piece of refractory or a bubble, would not give a fringe pattern.

A feasibility experiment was conducted with the damage tester operated with a 10us pulse. The glass for this experiment was known to contain particles whose diameter is of the order of 10 to $15 \mu$. The apparatus used for the experiment is shown schematically in Fig. 3. A Spectra-Physics Model 125 He-Ne laser with a power output of 20 milliwatts at $0.628 \mu$ made the holograms. The first hologram was made with the removable mirror and the removable light absorber out of the beam. These two elements were then replaced and the billet irradiated with the output of the damage tester. The second hologram was then quickly taken with the mirror and the light absorber again removed. The film used was AGFA-GEVAERT 8E70 with a resolution of 1500 lines/ $\mathrm{cm}$ and a sensitivity of $200 \mathrm{ergs} / \mathrm{cm}^{2}$. To avoid excessive noise from the nonlinear response of the film, by attenuation before the beam splitter an exposure of $3 \mu \mathrm{J} / \mathrm{cm}^{2}$ was used such as to give a $60 \%$ modulation on the film. The length of each of the exposures to the He-Ne light which was $1 \mathrm{~ms}$ and the time between irradiation with the $1.06 \mu$ beam and the second hologram was $40 \mathrm{~ms}$.

In Fig. 4 are shown a series of pictures taken of the reconstructed image in the neighborhood of an absorbing inclusion which was irradiated with $40 \mathrm{~J} / \mathrm{cm}^{2}$. Figure 4 is a series taken with the plane of the focal region of the microscope moved successively by $1 \mathrm{~mm}$ increments. In Frame $A$, the focal plane is $1 \mathrm{~mm}$ in front of the inclusion, in $\mathrm{B}$ in the plane of the inclusion, in C one millimeter behind the plane of the inclusion, and in D two millimeters behind the inclusion. In the plane of the best focus on the particle, two fringes are present which is indicative of absorption by the inclusion. The evidence in Fig. 4 was much more clearly indicated in the direct observation of the reconstructed image, because it was possible for the observer to focus through the plane of the particle and in this way clearly distinguish between particles with fringes and those without them. 
To identify particles of the order of 1 to $2 \mu$ in diameter would require much higher energies. Longer pulse times could be used so as to develop observable phase delays in the surrounding glass without damage.

\section{Recent Experimental Glass}

Three experimental melts have been made of low loss glass which appeared to be entirely free of inclusions. The largest melt was a two liter volume in which only about $1 / 3$ of the glass was sufficiently free of striae to be useful. This volume was swept with 100 to $120 \mathrm{~J} / \mathrm{cm}^{2}$ in $100 \mathrm{~ns}$ and no damage produced. In ten additional shots in which two CC's of glass are irradiated at up to $500 \mathrm{~J} / \mathrm{cm}^{2}$ in $100 \mathrm{~ns}$, no damage resulted. Self-tracking damage occurred at $500 \mathrm{~J} / \mathrm{cm}^{2}$ in an $80 \mathrm{~ns}$ pulse. The measured value of the loss coefficient was $0.2 \% / \mathrm{cm}$. This glass will not be commercially available until reasonably large volumes of striae free glass can be fabricated.

\section{Acknowledgement}

The author gratefully acknowledges the work done by C. Padula in testing the glass and the holographic work by $\mathrm{R}$. van Ligten, $J$. Levitt and $K$. Lawton. R. Hopper and D. Uhlmann are acknowledged for helpful discussions on the damage mechanisms due to inclusions. The cooperation of R. Woodcock, G. Young and E. Deeg is gratefully noted.

\section{References}

[1] Young, C. G. and Woodcock, R. F., "Laser Induced Damage in Glass," Damage in Laser Glass, A. J. Glass and A. H. Guenther, Eds., ASTM STP 469, American Society for Testing and Materials, 1969, pp. $84-99$.

[2] Deeg, E. W. and Silverberg, C. G. , "Melter for Optical Glasses," J. of Glass Manufacturing, April 1970.

\section{$1.8 \mathrm{mrad}$}

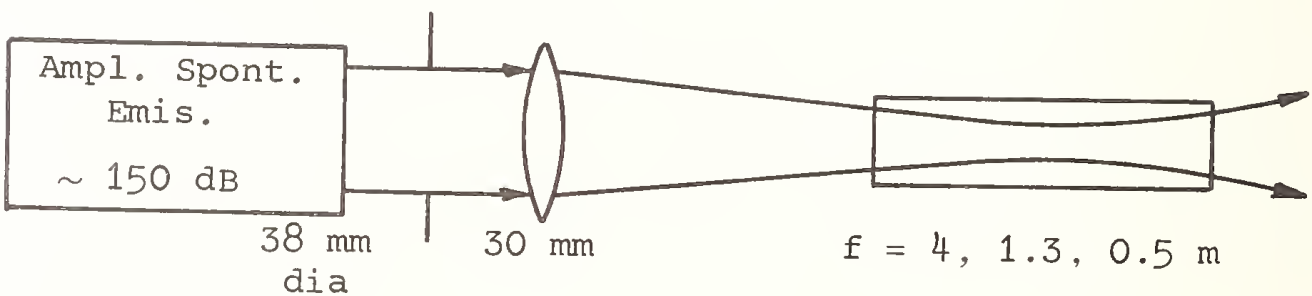

\begin{tabular}{|c|c|}
\hline PULSE DURATION & TOTAL ENERGY/PULSE \\
\hline $4-10 \mu \mathrm{S}$ & $80-100 \mathrm{~J}$ \\
$0.5-1.0 \mu \mathrm{s}$ & $55-80 \mathrm{~J}$ \\
$100 \mathrm{~ns}$ & $15-25 \mathrm{~J}$ \\
\hline
\end{tabular}

Fig. 1 Schematic of amplified spontaneous emission device used to test glass billets for inclusion damage. The total energy available for the various time domains are indicated in the table. The focal length for the lens used to focus into the billet decreased with decreasing energy in order to provide the necessary energy density
within the glass. 


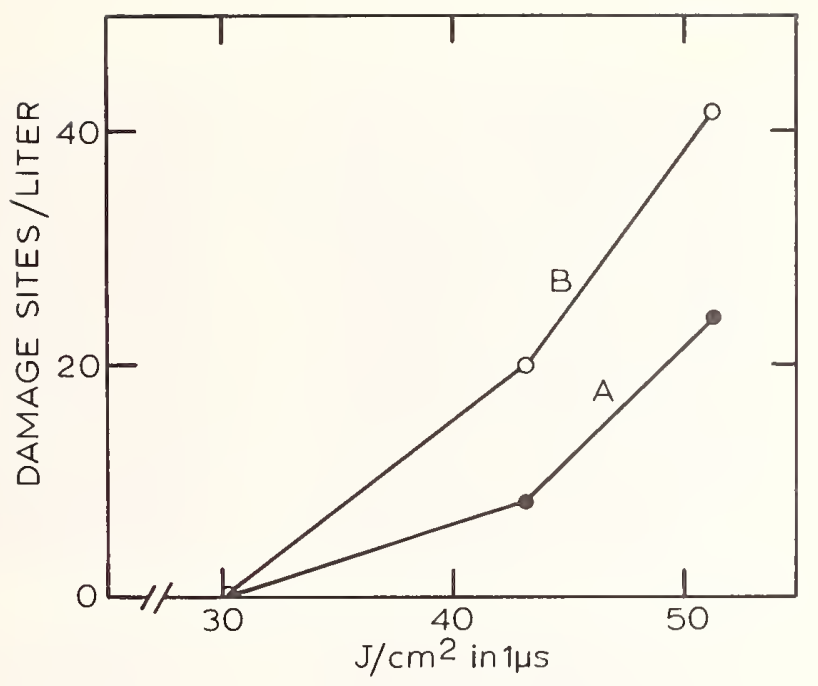

Fig. 2 The density of damage sites produced in currently available AO commercial laser glass. The glass that was tested had previously survived $80 \mathrm{~J} / \mathrm{cm}^{2}$ in $10 \mu \mathrm{s}$.

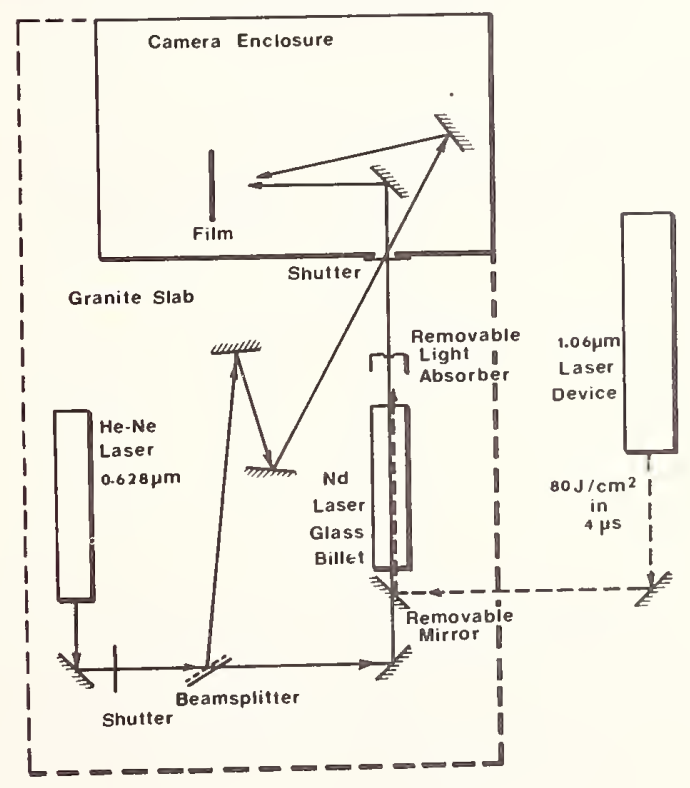

Fig. 3

Schematic of holographic apparatus for nondestructive identification of the positions of damaging inclusions. 

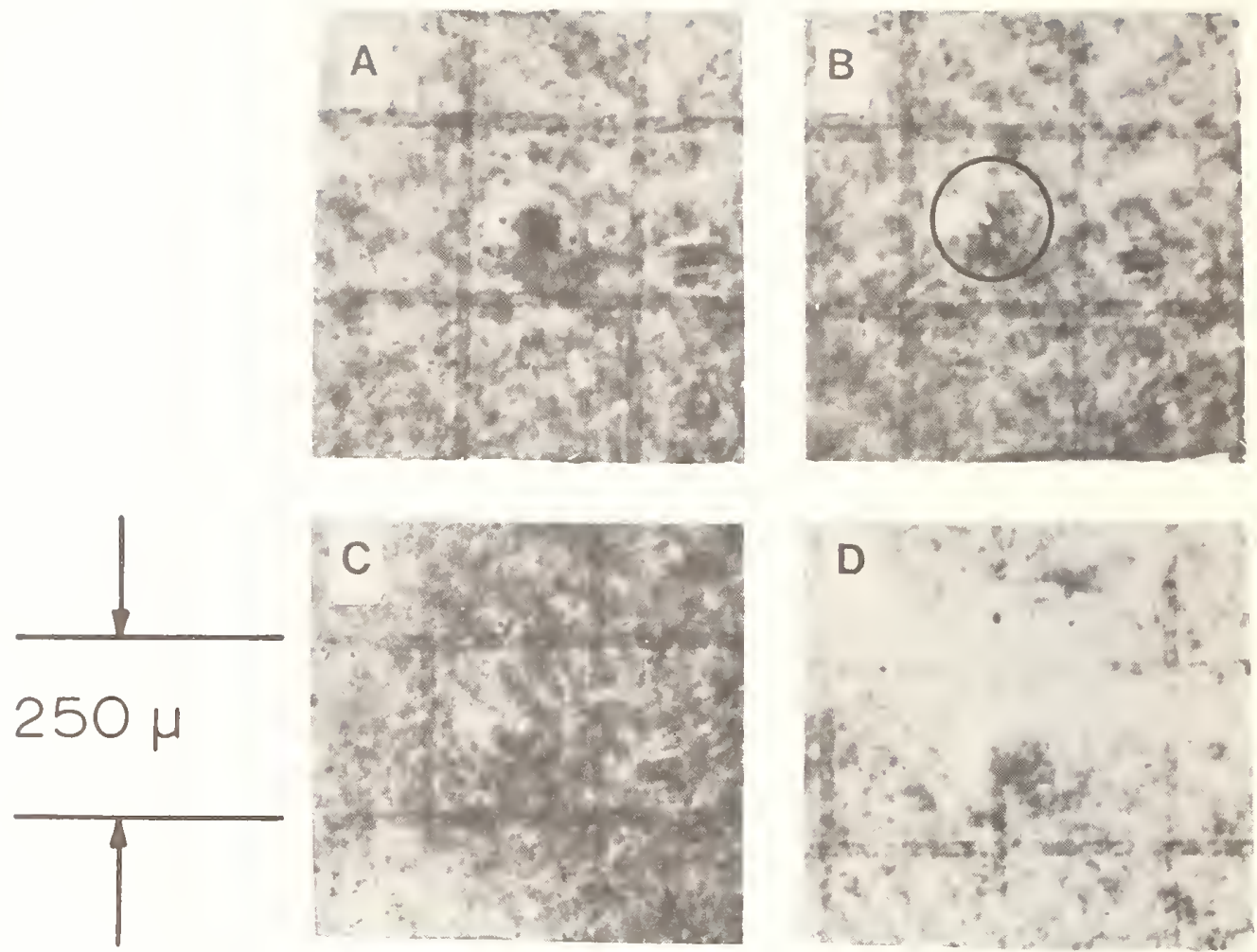

Fig. 4 Photographs of the reconstructed image of a hologram when focusing in various focal planes in the vicinity of an absorbing inclusion. Frame $A$ is $1 \mathrm{~mm}$ in front of the inclusion, $B$ at the inclusion, C $1 \mathrm{~mm}$ behind the inclusion and D $2 \mathrm{~mm}$ behind the inclusion.

COMMENTS ON PAPER BY ELIAS SNITZER

The measurements reported here were preliminary, and were designed to demonstrate the feasibility of using the holographic method to locate absorbing particles in the glass. At present, particles of diameters as small as 10 to $15 \mathrm{um}$ can be detected. These particles yield a distortion pattern of 2 fringes when irradiated ar a level of 40 joules $/ \mathrm{cm}^{2}$. To see particles of one $\mu m$ size, with the same sensitivity, would require 100 times as great an energy density. It is possible, with care, to increase the sensitivity of the present apparatus to detect a shift of a fraction of a fringe. The holographe exposure is taken 40 to $50 \mathrm{msec}$ after the heating pulse. The recording exposure duration is about one msec. It was felt that negligible cooling occurs on this time scale. Tests were run on unpumped samples only.

No fatigue effects were observed in ceramic-made glass, although older samples of glass made in platinum under a controlled atmosphere did exhibit fatigue effects. In all cases, the damage site grows with each subsequent exposure. 
Laser Glass Damage Testing

At Owens-Illinois

R. W. Beck

Owens-Illinois, Ine.

Toledo, Ohio

owens-Illinois is continuing to test a variety of glass samples to determine their damage threshold to laser irradiation.

Active damage threshold tests in the third amplifier stage of the owensIllinois oscillator-amplifier system indicate that selected samples may withstand peak energy densities of 50 joules/ $\mathrm{cm}^{2}$, for 50 nano-sec pulses. The increase in the peak energy densities for damage result from improved melting processes in the period from February of 1969 to February of 1970. The effect, thus far, is an $84 \%$ increase in the laser glass damage threshold.

Active life tests in the third amplifier stage at peak energy densities of 36 joules $/ \mathrm{cm}^{2}$ indicate that no degradation of the lasing properties, notably, efficiency, beam profile, pulse duration, and beam divergence occur for a 200 shot sequence. These results imply that for current state-of-the-art laser glass, system output degradation characteristics should at least be a part of any criterion for laser glass damage.

Passive damage tests on samples approximately $0.5 \mathrm{~cm}$ thick by $2 \mathrm{~cm}$ square indicate that micron to sub-micron inclusions are most easily damaged. A sample, which contained no visible inclusions at looX magnification with side lighting, did not damage at a peak energy density of 70 joules $/ \mathrm{cm}^{2}$.

Self-trapping and surface damage measurements on a $91 \mathrm{~cm}$ long unpumped sample resulted in no damage with an unfocused beam of 73 joules/ $\mathrm{cm}^{2}$ average energy density. With the sample placed ahead of the focus of a 2 meter lens, orange peel damage appeared on the output face at an average energy density of 136 joules $/ \mathrm{cm}^{2}$, pitting damage appeared at an average energy density of 144 joules/ $\mathrm{cm}^{2}$, and self-trapping damage occurred at an average energy density of 128 joules $/ \mathrm{cm}^{2}$. No input face damage was noted for a maximum average incident energy density of 140 joules $/ \mathrm{cm}^{2}$.

Key Words: Active damage threshold, inclusion damage, life test, passive damage threshold, self-trapping damage, surface damage.

\section{Introduction}

Laser glass testing at owens-Illinois is directed toward the characterization of laser glass damage, first, to provide the users of laser glass with reliable damage threshold data and, second, to intermally utilize the information for further laser glass quality improvements.

There are several possible mechanisms responsible for the observed internal damage in laser glass, such as absorption and subsequent heating, plasma formation from multiphoton absorption and avalanche breakdown, stimulated Brillouin scattering, and others. Each process exhibits its own characteristic dependence on several critical parameters, such as pulse duration, wavelength, beam profile, and the degree of focusing, and each process is in turn enhanced by self-trapping. This then presents a rather complex experimental environment and any valid testing program must define and control the many experimental conditions which prevail during damage testing. In this vain, the experimental configuration with which the damage measurements at owens-Illinois are performed and the techniques used to determine the experimental conditions are first defined. As the discussion proceeds, the experimental conditions which prevail during each damage measurement are indicated, noting such parameters as the lasing energy, pulse duration, beam profile, beam divergence, whether the beam is focused or unfocused, and whether the test sample is pumped or unpumped, which is referred to as active or passive testing. 


\section{Experimental Configuration and Measurement Techniques}

The principal test facility for the damage measurements, shown in Figure I, is basically a Qswitched, neodymium glass, laser oscillator-trebble amplifier system. The system is Brewstered and all rod diameters are $19 \mathrm{~mm}$. The rods are pumped with helical flashlamps and each lamp delivers a maximum of 4060 joules at $6.0 \mathrm{KV}$. The rotating resonant reflector, Q-switched, oscillator will deliver a maximum of from 10 to 12 joules, full aperture, in a 40 nano-sec pulse. To enhance the beam uniformity, an $11 \mathrm{~mm}$ major axis elliptical aperture is placed after the oscillator output mirror. About 5 joules passes through this aperture. The beam is then expanded and recollimated by a $1.5 \mathrm{X}$ telescope after which it passes through the three amplification stages. Elliptical apertures with $16 \mathrm{~mm}$ major axes are placed on the input faces of amplifiers one and two to lessen the probability of output face damage and to keep the beam away from the rod sidewalls.

The average energy density within the system is sampled both at the input and the output of the amplification stage using beam splitting components. The energy is monitored with TRG thermopiles and recorded on milli-volt strip chart recorders. The maximum average energy density wich the system will deliver is approximately 75 joules $/ \mathrm{cm}^{2}$.

The lasing pulse duration is monitored with a TRG 105B photodetector and a 519 Tektronix Oscilloscope and the system generally emits a 50 nano-sec pulse.

The system beam divergence is determined by burning exposed film placed loo cm behind a pinhole grid. The system typically has a slightly better beam divergence in the direction across the TIR roof edge than along the edge. For an oscillator cavity length of $135 \mathrm{~cm}$, the vertical and horizontal full angle beam divergences are 0.5 and 1.5 milli-radians for a 30 joule output, and 1.0 and 2.0 milliradians for a 60 joule output, and 1.0 and 2.0 milli-radians for a 90 joule output.

The device used to measure the lasing beam profile is a United Detector photodiode array consisting of 13 photodiodes in the form of a cross. The diodes are spaced on 3.8 mm centers and each diode is $2.0 \mathrm{~mm}$ in diameter. Each diode is part of a separate channel consisting of a light sensing circuit, an emitter follower transistor stage, and an RC storage network. The array is calibrated with uniform laser energy and the stored voltages are normalized to the center diode to provide relative profile measurements. As shown in Figure I, a wedged beam splitter samples the lasing output, a negative lens expands the beam to fill the array, and neutral density filters are inserted to control the beam intensity incident on the diodes. The distance from the output end of the third amplifier to the array is nominally $50 \mathrm{~cm}$. To convert voltage readings to energy densities in joules/ $\mathrm{cm}^{2}$, a computer program was written which integrates the volume contained by a surface generated from ll voltage measurements. This volume is then divided by $2 \mathrm{~cm}^{2}$, which represents the circular cross-section of the beam within the amplifier rods, and is equated to the measured average energy density. The resulting energy density equivalent to one stored volt is then applied to each measured capacitor voltage, completing the conversion to energy densities. Typically the profiles have peak to average energy density values of 1.3 , 1.2, and 1.1 for respective system energy outputs of 30, 60, and 90 joules. A typical profile for a system output of approximately 40 joules/ $\mathrm{cm}^{2}$ peak energy density is shown in Figure 2 .

In the damage statistics which follow, the damage thresholds will be given in both average joules/ $\mathrm{cm}^{2}$ and peak joules/ $\mathrm{cm}^{2}$, the conversion factors for which are obtained from profiles similar to the one shown, but recorded during the particular measurement.

Active damage thresholds are observed in samples pumped in the third amplifier stage. The occurrence of internal damage is noted by the presence of a visible inclusion with the sample illuminated with a microscope lamp, which easily detects $10 \mu$ size inclusions. Passive damage measurements are performed on samples placed external to laser system, either with or without the aid of focusing optics.

\section{Active Damage Tests}

During the course of the period from July of 1969 to the present, 18 third amplifier rods were actively damage threshold tested. The rods were selected equally from each of three melts between February of 1969 and February of 1970. Table I summarizes the damage results obtained for the three melts. The figure indicates both the average and the peak energy density at the threshold of damage for each rod tested. At the onset of damage no more than two damage sites were ever noted and these were generally in the order of from $100 \mu$ to $200 \mu$ in size. It is significant to note that the range of damage thresholds within a melt is of the same order as the melt average, indicating that the damage sites are not unifomly distributed throughout the melt either in size, location, or both. The highest damage thresholds obtained to date have been samples one and five of melt $\mathrm{C}$ which damaged at peak energy densities of 52.6 and 53.1 joules $/ \mathrm{cm}^{2}$ respectively. 
Table 1. Active Damage Summary

Melt "A" (February 1969)

\begin{tabular}{|c|c|c|}
\hline Sample No. & $\begin{array}{c}\text { Average Damage Threshold } \\
\text { (Joules }\left(\mathrm{cm}^{2} \text { ) }\right.\end{array}$ & $\begin{array}{c}\text { Peak Damage Threshold } \\
\text { (Joules }\left(\mathrm{cm}^{2} \text { ) }\right.\end{array}$ \\
\hline \multirow[t]{2}{*}{$\begin{array}{l}1 \\
2 \\
3 \\
4 \\
5 \\
6\end{array}$} & $\begin{array}{r}8.0 \\
10.8 \\
12.0 \\
6.8 \\
9.8 \\
23.9\end{array}$ & $\begin{array}{l}12.8 \\
17.3 \\
19.2 \\
10.9 \\
15.7 \\
31.1\end{array}$ \\
\hline & Average $=11.9$ & Average $=17.8$ \\
\hline \multirow{3}{*}{$\begin{array}{l}1 \\
2 \\
3 \\
4 \\
5 \\
6\end{array}$} & Melt "B" (october 1969) & \\
\hline & $\begin{array}{l}25.0 \\
15.0 \\
16.6 \\
18.8 \\
13.0 \\
16.4\end{array}$ & $\begin{array}{l}31.8 \\
20.3 \\
21.6 \\
25.9 \\
18.2 \\
25.7\end{array}$ \\
\hline & Average $=17.5$ & Average $=23.9$ \\
\hline \multirow[t]{2}{*}{$\begin{array}{l}1 \\
2 \\
3 \\
4 \\
5 \\
6\end{array}$} & $\begin{array}{c}\text { Melt "C" (February 1970) } \\
41.6 \\
13.8 \\
19.4 \\
26.1 \\
46.9 \\
13.8\end{array}$ & $\begin{array}{l}52.6 \\
16.6 \\
23.1 \\
33.1 \\
53.1 \\
18.6\end{array}$ \\
\hline & Average $=26.9$ & Average $=32.8$ \\
\hline
\end{tabular}

An analysis of the data also indicates that the average of the peak damage thresholds for melt $C$ is $84 \%$ better than the corresponding figure for melt A, indicating a significant improvement in melting capabilities in the time period February 1969 to February 1970. When the thermo-dynamic analysis of the melting environment is completed, it is hoped that the results will dictate further improvements in the ensuing months.

\section{Iife Test Results}

In the preceding measurements, laser glass damage is defined as the appearance of an inhomogeneous physical defect within the material resulting from the transmission of a laser pulse. An alternative definition is a degradation in the output performance of the laser system in terms of some system variable such as efficiency, beam profile, beam divergence, or pulse duration, and it is such a performance deterioration which is of most importance to the users of laser material.

To determine the significance of such a proposed degradation definition of laser glass damage, a rod from melt $B$ and a rod from melt $C$ were actively life-tested in the third amplifier stage for 200 shots each at a peak energy density of 36 joules/ $\mathrm{cm}^{2}$. No system deterioration in efficiency, beam profile, beam divergence, or pulse duration was noted in these tests. The rod from melt $B$ had a total of 11 inclusions at the end of the test, one having grown to $0.5 \mathrm{~mm}$, while the rod from melt $\mathrm{C}$ had only the original one inclusion at the end of the test, and the inclusion had not enlarged. These results strongly imply that for the current state-of-the-art of laser glass, output degradation characteristics should at least be a part of any criterion for laser glass damage.

\section{Passive Damage Measurements}

At the outset of the current laser damage program one of the primary goals was to generate inclusion size and type versus damage threshold data. To attain this goal small samples approximately 0.5 cm thick by $2 \mathrm{~cm}$ square were polished and mapped at $100 \mathrm{x}$ magnification with side lighting. The samples were then irradiated and remapped. The evaluation of many such samples concluded that such a technique is not practical. First, due to the small density of inclusions present in the material, large rolumes must be mapped to locate the desired inhomogeneities. Second, when inclusions are found they raxely damage when irradiated, rather, small micron to sub-micron inclusions which show up with side lighting produce the majority of the damage and wash out the original inclusion. Third, quite often damage 
results in areas where the glass was thought to be inclusion-free. Attempts are presently being made to locate the inclusions with holographic techniques, but if the lowest damaging inclusions are in fact those of a micron to sub-micron size, then even holography may not have the necessary resolving power

One test of the passive damage measurements worth noting was a sample which was visually inclusionfree at 100X magnification and subsequently showed no damage at a peak energy density of 70 joules/cm². This indicates that if the sample was indeed inclusion-free, then the damage threshold of homogenous glass is quite high,

\section{Self-Trapping And Surface Damage}

Preliminary self-trapping and surface damage measurements have been performed on a $91 \mathrm{~cm}$ long unpumped sample. For the first test, the sample was irradiated with the unforused output of the oscillator-amplifier system. With this configuration, a maximum average energy density of 73 joules/cm incident on the front surface of the sample produced neither surface or self-trapping damage. Assuming a worst case beam divergence of 2 milli-radians, this is equivalent to an energy density of 63 joules/cm? on the output face of the sample.

Having produced no damage with the unfocused beam, a 2 meter lens was placed in the system immediately after the third amplifier and the test sample was placed ahead of the focus with its front end $100 \mathrm{~cm}$ from the lens, as shown in Figure 3. As the output energy of the laser was increased, the first occurrence of damage was an orange peel effect on the output face of the test sample. The appearance of this type of damage is shown in Figures 4 and 5. In Figure 4, the surface is slightly out of focus to more effectively show the waviness of the surface while in Figure 5 the surface is in focus and both orange peel and pitting damage are apparent. Sampling the output surface at several locations showed that this type of damage occurs at an average energy density of 136 joules $/ \mathrm{cm}^{2}$.

As the output energy was further increased, pitting damage began to appear on the output face. The onset of such damage on the average occurred at an energy density of 144 joules/ $\mathrm{cm}^{2}$, somewhat above the level for the orange peel damage. The appearance of this type of damage at threshold was generally marked by the presence of crater type damage surrounded by a larger region of crazed glass. Several such sites are show in Figures 6, 7, and 8 as the damage progresses from threshold to several times threshold. No front surface damage was noted during these tests for a maximum average energy density of 140 joules $/ \mathrm{cm}^{2}$.

Throughout the surface damage testing, self-trapping damage was noted. The trapping damage occurred anywhere from the front end to the exit end of the billet and generally occurred only after exit surface damage was noted, such that the exit face damage does not appear to be trapping limited. The average trapping threshold was found to be 128 joules/ $\mathrm{cm}^{2}$. Figures 9 , 10 , and 11 are typical of the trapping damage noted. As shown, the bubble type damage characteristically has a diameter of a few tens of microns and the spacing between bubbles is generally not uniform. A summary of the selftrapping and surface damage results is show in Table 2. The table primarily indicates the rather

Table 2. Self-Trapping and Surface Damage Results

\begin{tabular}{|c|c|c|}
\hline $\begin{array}{c}\text { Orange Peel } \\
\text { (Average Joules/cm²) }\end{array}$ & $\begin{array}{c}\text { Surface Pitting } \\
\text { (Average Joules/cm } 2)\end{array}$ & $\begin{array}{c}\text { Trapping } \\
\text { (Average Joules/cm } 2)\end{array}$ \\
\hline 134 & 123 & 83 \\
171 & 154 & 63 \\
112 & 150 & 170 \\
113 & 150 & 194 \\
147 & & \\
\hline
\end{tabular}

broad variation in the damage results, especially those related to the trapping threshold which varied from 63 to 194 joules $/ \mathrm{cm}^{2}$. Because of these rather large variations, this data should be considered as tentative only. Further tests are presently being prepared and in the near future the range for the damage threshold values will hopefully be narrowed. It cannot be ignored, however, that these damage levels are generally higher than those previously reported by others.

\section{Future Plans}

The planned research program for the next twelve months can be conveniently broken into four main phases as shown in Table 3 . 
1. Inclusion damage in the 1 to 5 nano-sec time domain.

2. Thermodynamics of the melting of laser glass in platinum.

3. Kinetics of self-trapping induced stress patterns.

4. Surface damage characterization.

The inclusion type damage measurements in the 1 to 5 nano-sec time domain will follow the same general procedure as that previously described for the 50 nano-sec work. Active tests will be performed in the third amplifier stage, while passive tests will be performed on unpumped samples exterior to the oscillator-amplifier system. The attainment of accurate data in this time domain together with the 50 nano-sec results should yield experimental information as to the effect of the time parameter in laser glass inclusion damage.

In the thermodynamic study the activities for oxides in laser glass and metals in platinum will be determined to assess the feasibility of melting laser glass in platinum for various partial pressures of oxygen. The basic problem is to lower the Po2 as low as possible without reducing the laser melt oxides and allowing the metals to attack the platinum. The one shortcoming of this approach is that it does not include the kinetics of the reactions so that after the activities are determined, some experimental work will be necessary to isolate the optimum melting conditions.

The kinetics of self-trapping induced stress patterns will be observed using the techniques of interferometric holography. The hologram will be produced with a 10 to 15 nano-sec ruby pulse at different time intervals during a 100 to 150 nano-sec pulse from our neodymium oscillator-amplifier system. The laser induced stress patterns and their temporal evolution will be observed as a function of the laser energy and the sample physical parameters which could be relevant to self-trapping (such as density, Young's modulus, refractive index, and others). Possible correlations of the stress pattern formation and evolution to the physical parameters will be explored with a goal to delineate these parameters which are relevant to self-trapping. Since self-trapping appears to be the precursor of laser glass damage, such a characterization would appear to be a meaningful contribution.

The fourth and final phase of the program will be to investigate those factors which may be expected to alter laser glass surface damage. Based upon the ambiguity as to whether such damage is caused by themal or mechanical failure, the effects of such processes as etching, vacuum drying, and mechanical strengthening will be determined. In addition to quantifying the relationship of each process to the damage threshold, the long term benefits of each process will also be investigated.

\section{Acknowledgements}

The support of the Advanced Research Projects Agency of the Department of Defense is gratefully acknowledged for both the current and succeeding twelve month programs. I would also like to acknowledge many fruitful discussions with Haynes Lee and Alex Glass, and I am most grateful to Ted Phillips who spent many endless hours obtaining the data for this paper.

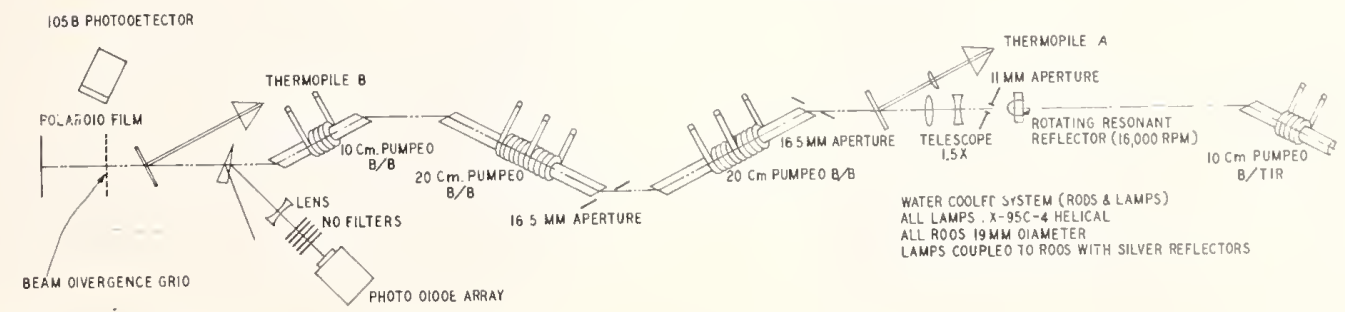



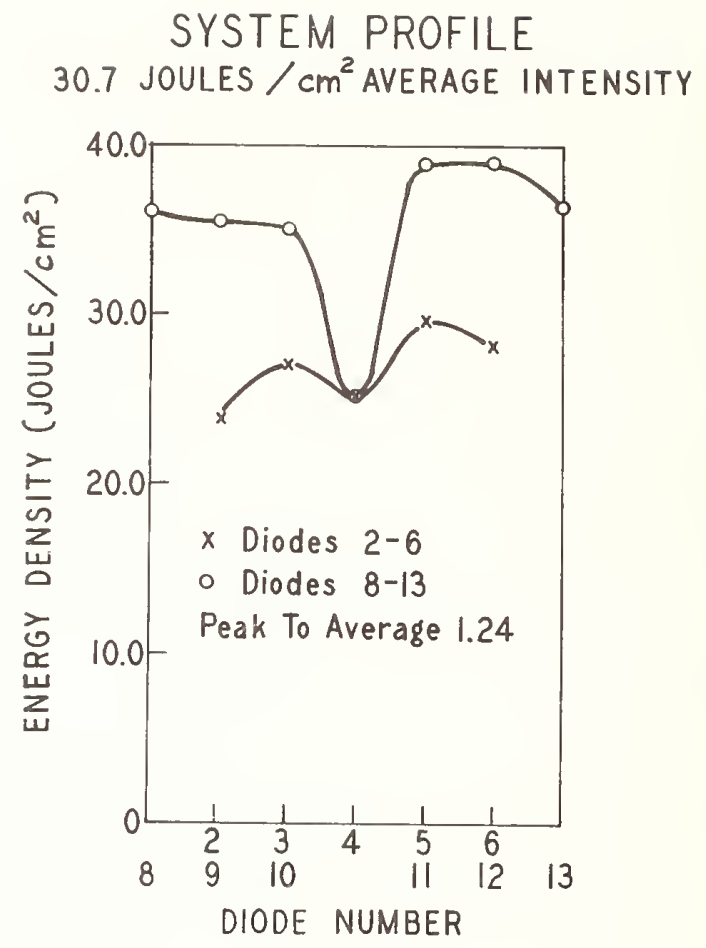

Fig. 2 System Profile - 30.7 Joules $\mathrm{cm}^{2}$ Average Intensity.

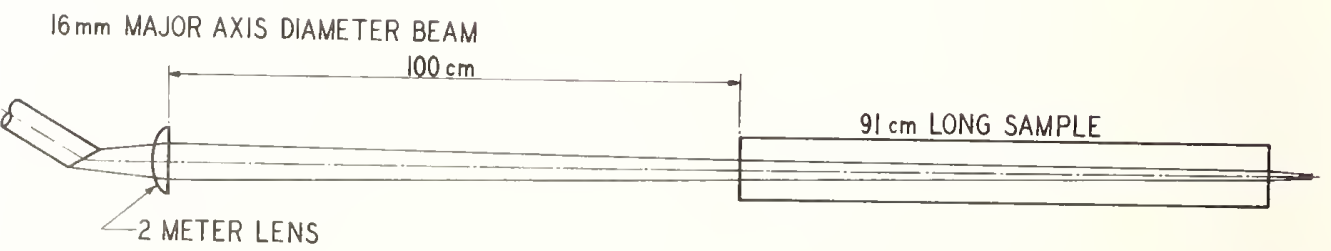

PULSE DURATION $50 \times 10^{-9}$ SECONDS

BEAM DIVERGENCE $2 \times 10^{-3}$ RADIANS

Fig. 3 Self-trapping and Surface Damage Experimental Arrangement 


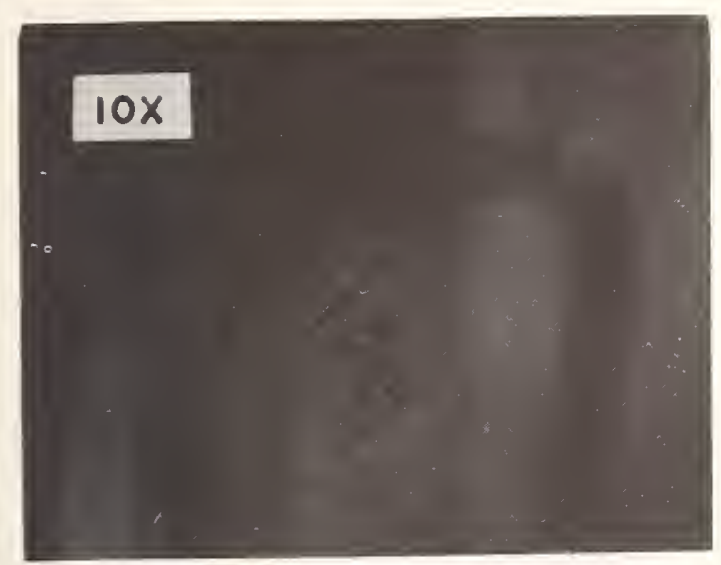

Fig. 4 Orange Peel Damage

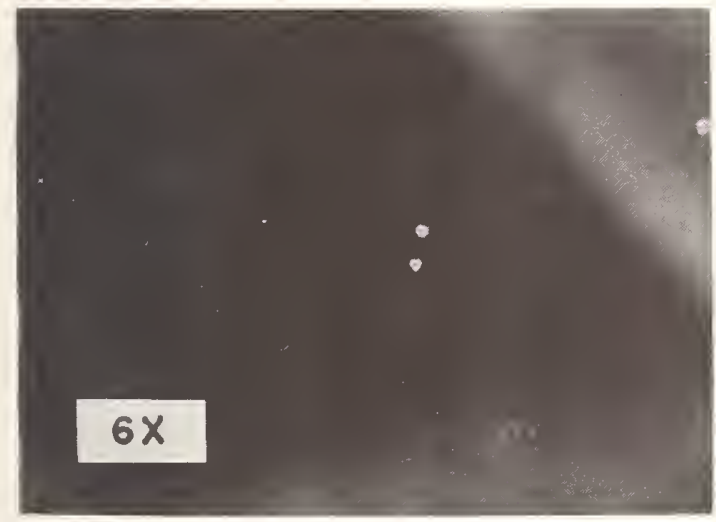

Fig. 6 Pitting Damage

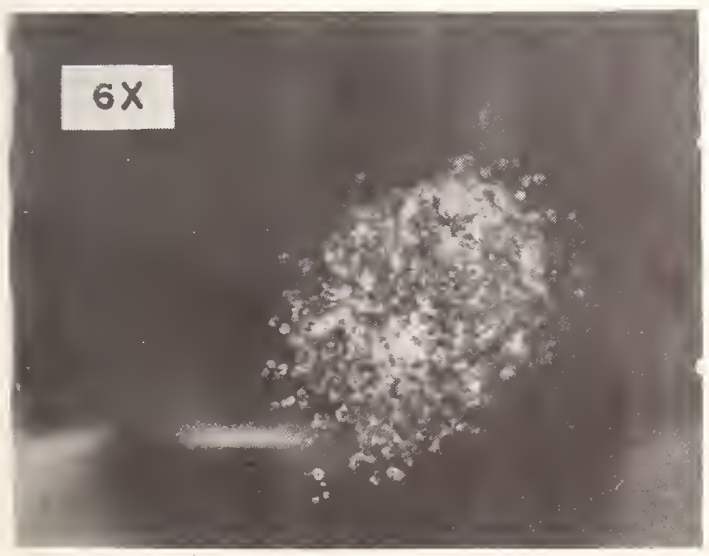

Fig. 8 Pitting Damage

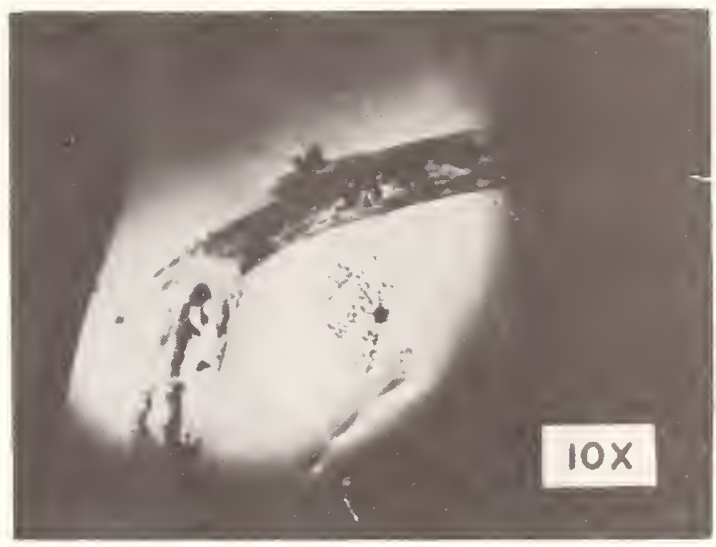

Fig. 5 Orange Peel Damage

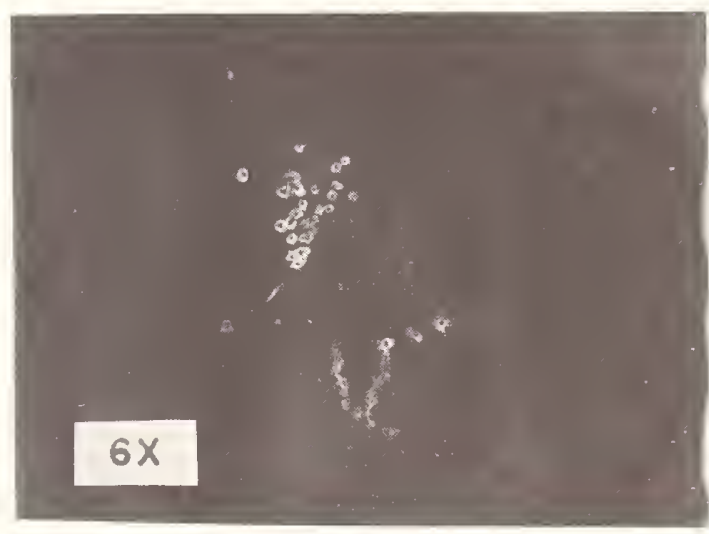

Fig. 7 Pitting Damage

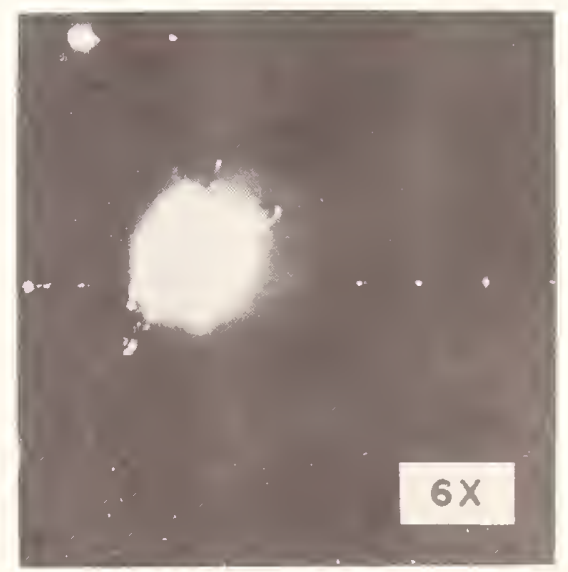

Fig. 9 Trapping Damage 


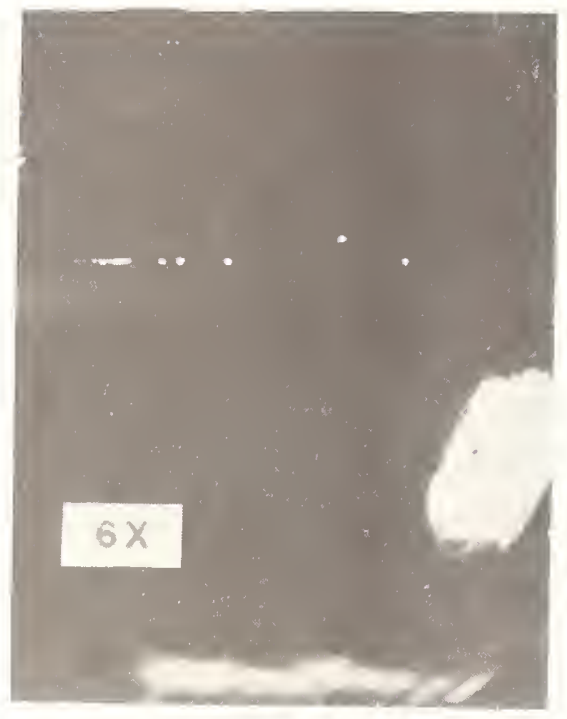

Fig. 10 Trapping Damage

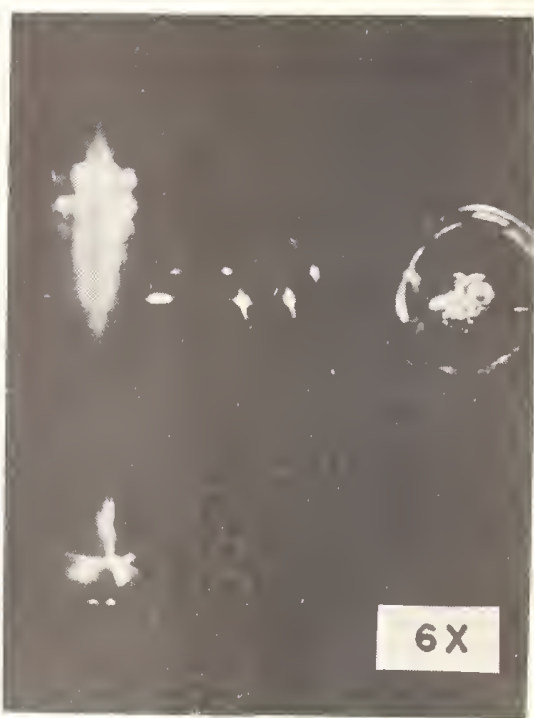

Fig. 11 Trapping Damage

COMMENTS ON PAPER BY ROBERT BECK

Due to the method used to measure the intensity profile, intensity data were taken oney at discrete points along perpendicular axes, rather than continously. No detailed fine structure was soivable b' this method. The ciiode array consisted of 8 diodes, each with a diameter of 80 thousandths of an inch, spaced 2 mo apart along each axis. Only the intense central portion of the beam was measured. The beam was elliptical due to the use of a Brewster-angle system, with a major diameter of $16 \mathrm{~mm}$. The intensity at the focal spot within the glass sample was computed from the recordea intensity profile, known focal length and measured total energy.

The $91 \mathrm{~cm}$ long sample was moved towards the focal spot, to increase the energy density within the sample. At a given sample position, the laser energy was increased until trapping danage was seen. Surface damage often occurred at the output face of the sample prior to the appearance of trapping danage. No surface danage was seen at the ertrance face of the sample at energy densities up to 140 joules $/ \mathrm{cm}^{2}$.

Cark Pitha of AFCRL reported seeing trapping damage in sapphire mirrors accompanied by "orangepeel" damage at the exit surface. Mr. Beck reported that in his tests, trapping near the exit face of the sample generakly was accompanied by pitting at the surface rather than "orange-peel" damage. 
Thomas H. DeRieux and John M. McMahon

Naval Research Laboratory

Washington, D. C. 20390

Damage thresholds exhibited by several different laser glasses are reported using the VD 320 system. The performance characteristics of these glasses when operated in the VD 320 laser is discussed. Of particular importance was the anomalous gain noted when very high population inversion was attempted as well as severe pulse broadening.

Key Words: Glass damage, laser amplifiers, ceramic hosts, optical gain, laser characteristics, pulse broadening.

\section{Introduction}

This report covers activities in laser glass evaluation during the past year. Test reports are included on test series on Owens-Illinois and American Optical laser glasses. During this period, the CGE VD 320 was modified by the addition of another amplifier to produce 115-joule, 30-nanosecond pulses. With this system $4 \mathrm{~cm}^{2}$ areas can be tested at fluxes in excess of 20 joules/cm ${ }^{2}$ Fairly complete tests on presently available oI laser glass were completed during this period. Some limited (and promising) tests were conducted on American Optical glass but the planned extensive test series has had to be postponed at American Optical's request.

No samples of Schott laser glass were tested during this period because an improved glass was supposed to be in the works. This glass has recently been announced and a rod is being purchased. The technical data supplied by Dr. Neuroth (Schott Jenaer Glaswerk, Mainz, DFR) shows this glass to be very similar to A00835 in its basic properties with a very low claimed loss coefficient $\left(\sim 1 \times 10^{-3} \mathrm{~cm}^{-1}\right)$.

Preliminary tests on a sintered ceramic neodymnium laser host, Yttralox from General Electric, are being reported separately.

The CGE VD320 laser system has in excess of four thousand shots at this point and has performed well throughout. No failures have occurred in the optical train although there have been a number of faults in the electronic system. These have in general been random component failures rather than a systematic degradation of the system. There have also been some failures due to poor original assembly techniques (e.g. HV connectors crimped but not soldered). The French high voltage leads have been replaced with Amphenol 21-412 cable to ensure long term alignment stability of the laser heads. The output face of the final amplifier was repolished after $\sim 3000$ shots as the surface had degraded somewhat, presumably because of residual scarring left by exploded dust particles. The degradation appeared to be rather slight and was only visible with strong illumination on the surface.

\section{Owens-Illinois Laser Glass}

Early in the fall a set of laser rods was received from Owens-Illinois for all three stages of the CGE laser. The sequence followed was to first tune up the oscillator then the first amplifier and finally the last amplifier.

It was expected that the oscillator would require less pump energy because of the larger induced emission coefficient and lower passive loss in the glass. In fact the oscillator could be tuned up to produce a 30-35 nanosecond pulse with about $65 \%$ of the pump energy required for the French glass. It was found necessary to aperture the rod to $14 \mathrm{~mm}$ to suppress scattering from the rod barrel. Even under these conditions the optimum beam divergence from the oscillator was approximately twice what it was with the French glass. In addition, the near field fringes lacked contrast and the far-field pattern was more Lorentzian than Gaussian in shape. This behavior is characteristic of a situation where there is excessive small angle scattering in the laser rod. [1] 
It should be noted that there was no selection procedure performed by Owens-Illinois on this laser rod which is in direct opposition to the procedure employed by CGE. The French fabricate a number of rods and test all of them in a standard oscillator to select those which perform at or better than their beam divergence specification (late in 1968, this represented less than $20 \%$ of the rods fabricated [2]). Homogeneity in an oscillator rod is especially important since it is a multipass situation and an active testing specification may be necessary for low divergence systems. It was decided to use the oscillator rod as the larger beam spread ( 0.5 mr) was still acceptable for the test series.

In setting up the first amplifier of the system consideration was given to the fact that the fluorescence lifetime $(\sim 300 \mathrm{\mu sec})$ of the Owens-I1linois was much shorter than the pump pulse duration $(750 \mathrm{\mu sec})$. For this reason the bank capacitance was varied to observe the effect of pump pulse width on the performance. The peak voltage ratings of the capacitors, $12 \mathrm{kV}, 1 \mathrm{imited}$ the energy at the shorter durations to $8 \mathrm{~kJ}$ at $360 \mu \mathrm{sec}$ and $12 \mathrm{~kJ}$ at $480 \mu \mathrm{sec}$, compared to the normal $20 \mathrm{~kJ}$ at $750 \mathrm{usec}$. Figure 1 shows the results of this exercise. The outputs at 12 and $20 \mathrm{~kJ}$ were identical and it appeared that $10 \mathrm{~kJ}$ at $360 \mathrm{\mu sec}$ would also have given the same output. Removing the potassium chromate ultraviolet cladding and using distilled water alone increased the output by 5-10\% in all cases at these levels although this was not true at higher levels. The curves all had very similar shapes. In terms of a model relating the energy storage efficiency to the ratio of fluorescence intensity to pulse duration including changes in the spectral distribution of flashlamp radiation at different loadings on the lamp, the results were somewhat more dependent on pulse width than was expected, in that somewhat more energy was required at the longer pulse widths than would be expected from the model. This may be due to amplified spontaneous emission at the fairly high energy densities stored in this $\operatorname{rod}\left(\sim 1 \mathrm{~J} / \mathrm{cm}^{3}\right)$. This amplifier rod appeared to be of excellent optical quality and did not noticably degrade the beam divergence.

The output of the device was run up to $13 \mathrm{~J} / \mathrm{cm}^{2}$ average and $20 \mathrm{~J} / \mathrm{cm}^{2}$ peak with no signs of damage.

Experiments were also performed at $1,15 \mu$ and $6328 \AA$ to determine whether or not there was any appreciable pump induced absorption at these wavelengths. The experiments to date indicate that any pump induced loss must be less than $0.002 \mathrm{~cm}^{-1}$ at $1.15 \mu$ and $6328 \AA$ at pumping 1 evels up to $100 \mathrm{~J} / \mathrm{cm}^{3}$ (lamp energy per cm of laser material).

It was realized from this and other data that the VD 320 did not have a high enough output energy density to function as a damage testing system for presently available laser glasses and an additional amplifier was fabricated to raise the total output to 120 joules. This amplifier was designed for $32 \mathrm{~mm} \times 250 \mathrm{~mm}$ long laser rods. The previous data on the effects of pulse width variation led us to choose a $500 \mu \mathrm{sec}$ duration pump pulse. Flashlamp design data [3,4] 1ed us to choose a maximum input of 7500 joules to each helical lamp at $17.3 \mathrm{kV}$. The French lamp life data gives an expected lamp life to 10,000 shots for this system.

The expanded system was then run with French glass in the first three stages and the 0-I rod in the final state. The amplifier was run both with and without the potassium chromate cladding solution. Figure 8 shows the measured small signal gain coefficients (on- $Y$ ) $\ell$ for both cases. Without the cladding $(\sigma n-\gamma) \ell$ was about $5 \%$ higher than with the 2.5 gm/liter solution. The measured output energies vs pump input are shown in Fig. 9. Here the total output was higher with the cladding by about 5 joules ( $115 \mathrm{~J}$ vs $110 \mathrm{~J}$ ). The system was also run with the intermediate expander (between 23 and $32 \mathrm{~mm}$ stages) removed and the output over $4 \mathrm{~cm}$ found to be slightly in excess of $21 \mathrm{~J} / \mathrm{cm}^{2}$. During all tests at and around this level no damage was noted which implied that the density of inclusions which would explode under $20 \mathrm{~J} / \mathrm{cm}^{2}$ was 1 ess than 1 per $50 \mathrm{~cm}^{3}$ of glass.

Figure 10 shows the input-output curves for this amplifier at 5, 10, and $15 \mathrm{~kJ}$ pump energies.

Several interesting facts were observed about the operation of this amplifier:

1. The energy storage efficiency (joules of inversion per joule of pump energy) decreased markedly at higher pump energies. It was $1.4 \%$ at $5 \mathrm{~kJ}, 1.1 \%$ at $10 \mathrm{~kJ}$ and $0.9 \%$ at $15 \mathrm{~kJ}$. From the model we would have expected $1.40 \%, 1.27 \%$, and $1.17 \%$ at the three input energies.

2. The fluroescence intensity at $30^{\circ}$ to the optical axis (Fig. 8) was nonlinear. It could be linearized by use of the small signal gain coefficient and an effective length. Figure 11 shows the linearized results for an effective length of $11 \mathrm{~cm}$. This is a longer length than the direct view of this detector (i.e. the light must have been scattered off the wall).

3. The differences in input-output curves and small signal gains with and without the potassium chromate ultraviolet cladding were suggestive of the original active loss hypothesis. 
4. With the beam expander removed the input to the last amplifier was $\sim 1.5$ times the saturation flux and the output was $\sim 3$ times the saturation flux. The pulse shapes and timing were measured using ITT FW photodiodes and the results are shown in Fig. 12. Not only does the pulse leading edge move forward in time with a "phase" velocity of $\sim 25$ c (which is an effect first noted by Basov and Zuev in ruby [6]) but the pulse also broadens by $\sim 70 \%$. The theoretical results of Franz and Nodvik [7] would predict a broadening of no greater than $30 \%$ for this situation.

Several approaches are being pursued to understand these results:

1. The model of Xenon lamps pumping neodymnium laser material is in the process of experimental validation to insure that it accurately represents the actual physical situation as part of another program.

2. The effect of axial and near axial superradiant coupling between the oscillator and the amplifiers has been investigated by placing saturable absorbers between stages of the laser. These experiments showed no difference with or without the dye cells. The results of a simplified calculation for fluroescence lifetime shortening due to superradiance indicate that for our case, this effect is negligible in the rod itself.

3. Experiments are underway to evaluate the role played by transverse superradiance and whispering modes in the pump cavity in limiting the peak inversion achievable. This will be done by placing a $1.06 \mu$ absorber between the flashlamps and laser rod and evaluating the change in small signal gain.

4. The active loss coefficient will be measured in a more favorable experiment with improved $\mathrm{s} / \mathrm{n}$ ratio. Mirrors have been obtained to run our spectra-Physics Model 125 at $1.06 \mu$, $1.08 \mu$, or $1.15 \mu$ for this experiment. In addition, low noise PIN diode detector-amplifier combinations have been designed and fabricated to give $\mathrm{s} / \mathrm{n}$ better than 5000:1 at $1 \mathrm{MHz}$ bandwidth.

5. Duston's results giving a lower laser level cross relaxation time of $\sim 11$ nsec for the OI glass [7] could affect both the extraction efficiency and the pulse shape. Our computer programs show that the measured pulse broadening is consistent with $\tau_{L}-10$ nsec but some additional active loss still appears to be present.

6. The result of Vance shows that in at least one laser glass excited state absorption from the upper laser level is an important factor in reducing the gain. [8] This effect needs to be evaluated for OI glass in that physically it exhibits appropriate behavior.

\section{American Optical Laser Glass}

In November, American Optical told us that they had several amplifier rods to test. Mr. Charles Padula accompanied the rods and assisted in the experiments. Two rods were brought to NRL and time permitted detailed measurements only upon one of these. One rod was a standard C 835 glass and the other was a new type, number 2378 doped with Cerium $(\sim 2$ wt. \%). Both rods had numerous bubles.

Figure 13 shows the results obtained with the new glass in the second amplifier. The Cerium addition seemed to help only at the low pumping level. No inclusion damage was noted in this case. Figure 13 corresponds to the case where French glass was used in the oscillator. The system was also run with an OI oscillator and in this case the small signal gain coefficient was only half as large implying the $\sigma(1.0621)=0.5 \sigma(1.057)$ for the AO glass. This is lower than Duston's measurement of the relative fluorescence intensity profile would imply.

The passive loss coefficient was measured after $\sim 50$ shots and found to be $\sim 1.9 \times 10^{-3}$ $\pm 2 \times 10^{-4} \mathrm{~cm}^{-1}$. C. Padula had measured the passive loss at AO before running the rods and found $Y \sim(1.3 \pm .2) \times 10^{-3} \mathrm{~cm}^{-1}$. After returning to American Optical he measured this value and found, on the same apparatus, it to be $(1.8 \pm .2) \times 10^{-3} \mathrm{~cm}^{-1} \cdot[9]$

It was hoped to run a further test series with $A O$ glass in the spring out several difficulties prevented this. AO found that their testing procedure was not relevant to operation at 30 nanoseconds. and, is, at present, understandably unwilling to submit any more glass for testing until they have established satisfactory testing procedures. Hopefully, they will be back in business by the end of the summer.

\section{Future Plans}

Several more amplifiers are being fabricated in-house to allow the testing of glass at levels up to $40 \mathrm{~J} / \mathrm{cm}^{2}$. These should be ready by the middle of the summer. 
The results to date on the Owens-Illinois ED-2 laser glass are that:

1. Large volume of glass can be fabricated with a damage threshold above $20 \mathrm{~J} / \mathrm{cm}^{2}$ for Q-switched pulses.

2. The absorption loss was typically $\sim 2 \times 10^{-3} \mathrm{~cm}^{-1}$ for the glass tested but there seem to be some problems in obtaining large rods with good optical quality.

3. The active (pumped) performance of this glass is still not completely understood.

The results to date on the American-optical laser glass are that:

1. The sample tested withstood fluxes of $15-20 \mathrm{~J} / \mathrm{cm}^{2}$ without damage.

2. The net optical quality left something to be desired in that there were numerous bubbles in the glass which might be a problem in a very high gain amplifier train. The total loss coefficient was of the same order as for the Owens-Illinois laser glass $\left(\sim 1.9 \times 10^{-3} \mathrm{~cm}^{-1}\right)$.

3. The overlap of the two glasses was investigated and it was found that the induced emission coefficient of the AO 2378 glass at 1.0621. where OI glass lasers was $\sim 50 \%$ of the peak value $\left(1.6 \times 10^{-20} \mathrm{~cm}^{2}\right) \cdot[10]$

It would therefore not be recommended to have an OI oscillator with AO amplifiers but our earlier result on the OI glass pumped by the French (which has the same peak fluorescence wavelength as the AO glass) indicates that the reverse setup; AO, French or Schott oscillator and OI amplifiers would work as well as.with an oI oscillator.[11]

\section{References}

[1] McMahon, J.M., Laser Mode Selection with a Slowly Opened Q-Switch, IEEE, JQE, QE-5, 10 , October 1.969 , pp. 489-495.

[2] Sturel, J. (CGE), private communication.

[3] Flash Pour Barreau \& 32 mm" Centre De Richarches, Companie' General d' Electricite', Division Technique Report JS/AL - No. 66-A-149.

[4] Emmett, J. L. and Markievicz, J. P., Design of Flashlamp Driving Circuits, IEEE JQE, Vol. QE-2, No. 11, November 1966, pp. 707 , 711 .

[5] Ambartsumyan, R. V., Basov, N. G., Zuev, V.S., Kryukov, P. G., and Letokhov, V. S., IEEE, JQE, Vol. QE-2, 9, September 1966, pp. 436-441.

[6] Franz, L. and Nodvik, J., Theory of Pulse Propagation in a Laser Amplifier, J. Appl . Phys. 342346 (1963).
[7] Duston, D. K., Factors Influencing the Gain in Neodymnium Glass, Ph.D. Thesis, 1969, Renassaleur Polytechnic Institute, p. 125.

[8] Vance, M. E., Saturation and Excited State Absorption in Neodymium Laser Glass, IEEE, $\mathrm{JQE}, \mathrm{QE}-6$, 5, May 1970, pp. 249-253.

[9] Duston, D. K., Ibid., Fig: 4-3.

[10] Padula, C., (American Optica1) private communication.

[11] Young, C. G., Kantorski, J. W., Padula, C. F. and La Marre, D. A., paper number 10-1, Conference on Laser Engineering and Applications, May 26-28, 1969, Washington, D. C.

[12] McMahon, J. M., Glass Laser Materials Testing at the Naval Research Laboratory, Damage in Laser Glass, ASTM STP 469, (1969) pp. 117127. 


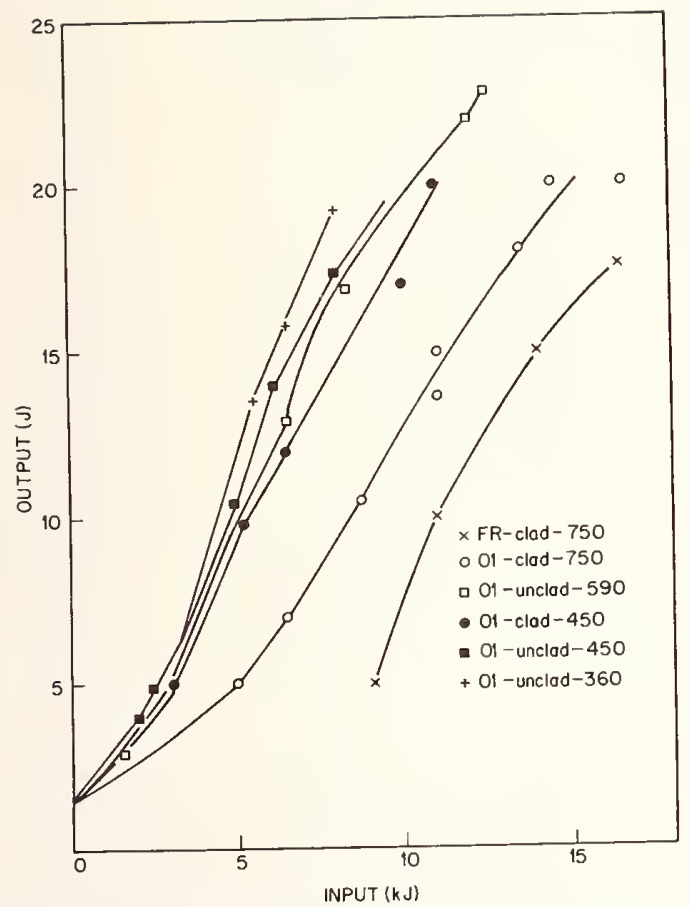

Fig. 1 Laser output of the intermediate amplifier $(23 \mathrm{~mm})$ of the $C G E$ laser with Owens-Illinois glass as a function of pump pulse energy with pump pulse with width varied. Also shown is the output with and without a ultraviolet absorber ( $2.5 \mathrm{gm} /$ liter of potassium chromate). Input was 2.5-joule, 30-nanosecond pulse.

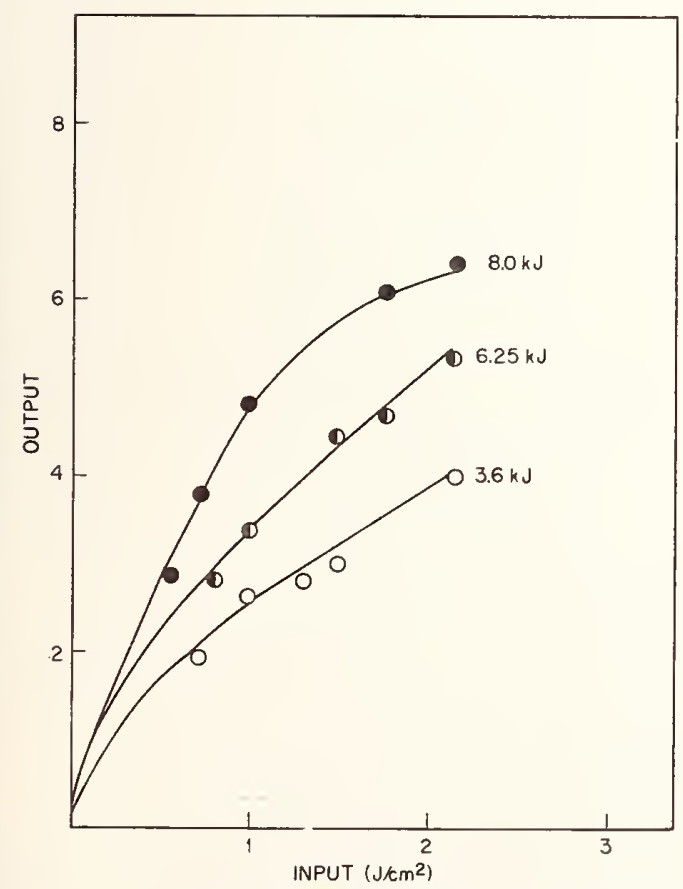

Fig. 3 Laser input vs laser output for the second amplifier with a $480 \mu \mathrm{sec}$ pump pulse at $3.6,6.25$, and $8.0 \mathrm{~kJ}$ pump energies.

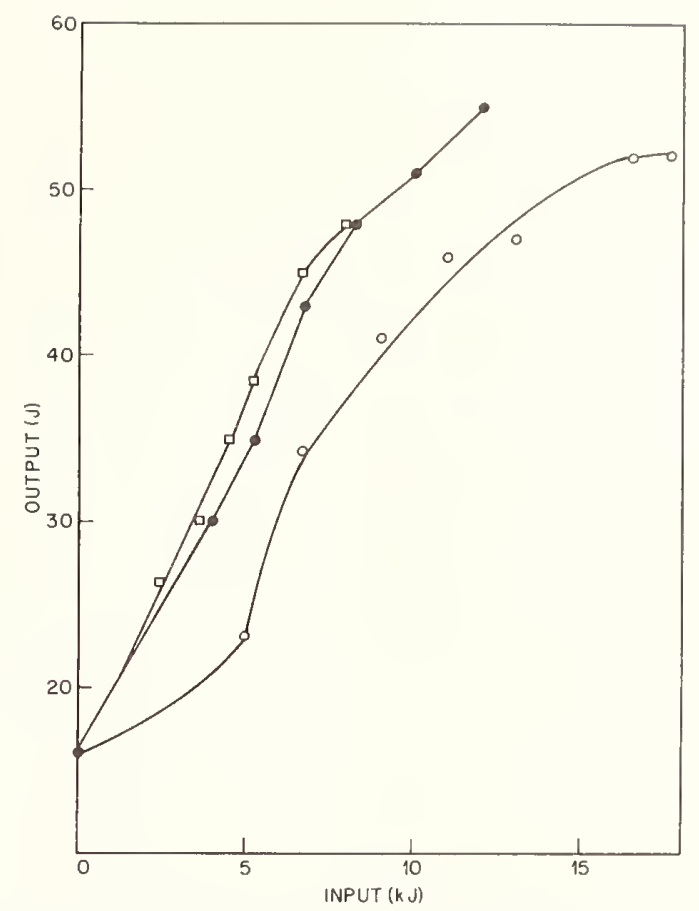

Fig. 2 Laser output of second amplifier (32 mm diameter) of CGE laser with Owens-Illinois glass vs pump energy for various pump pulse widths. Input was 19-joule, 30-nanosecond pulse.

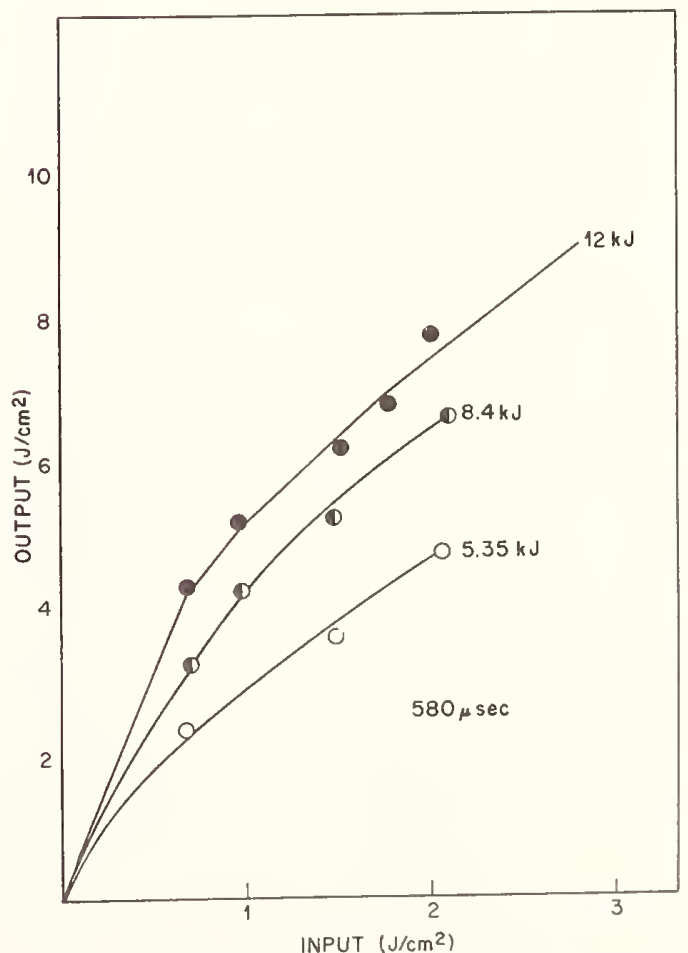

Fig. 4 Laser input vs laser output for the second amplifier with a $580 \mathrm{Hsec}$ pump pulse at $5.35,8.4$, and $12.0 \mathrm{~kJ}$ pump energies. 


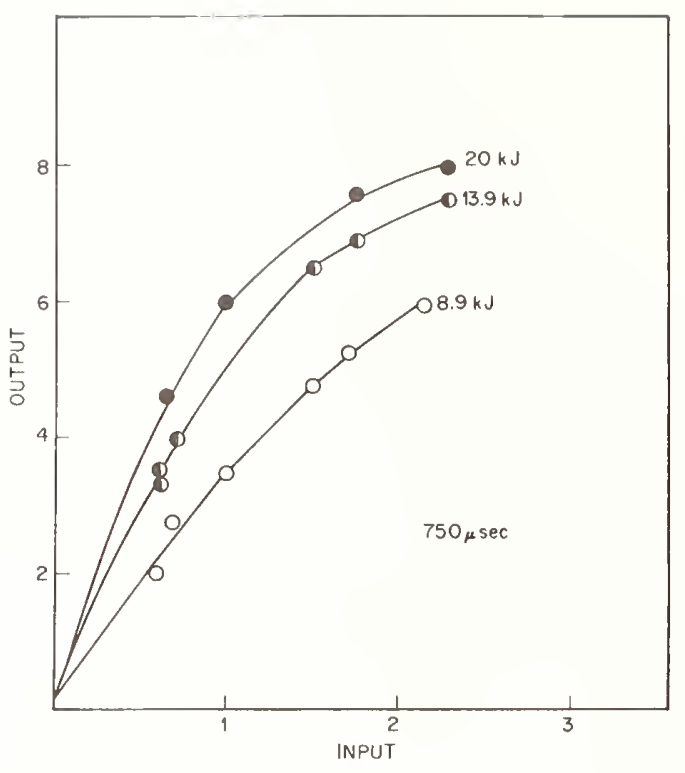

Fig. 5 Laser input vs laser output for the second amplifier with a $750 \mu \mathrm{sec}$ pump pulse at $8.9,13.9$, and $20 \mathrm{~kJ}$ pump energies.

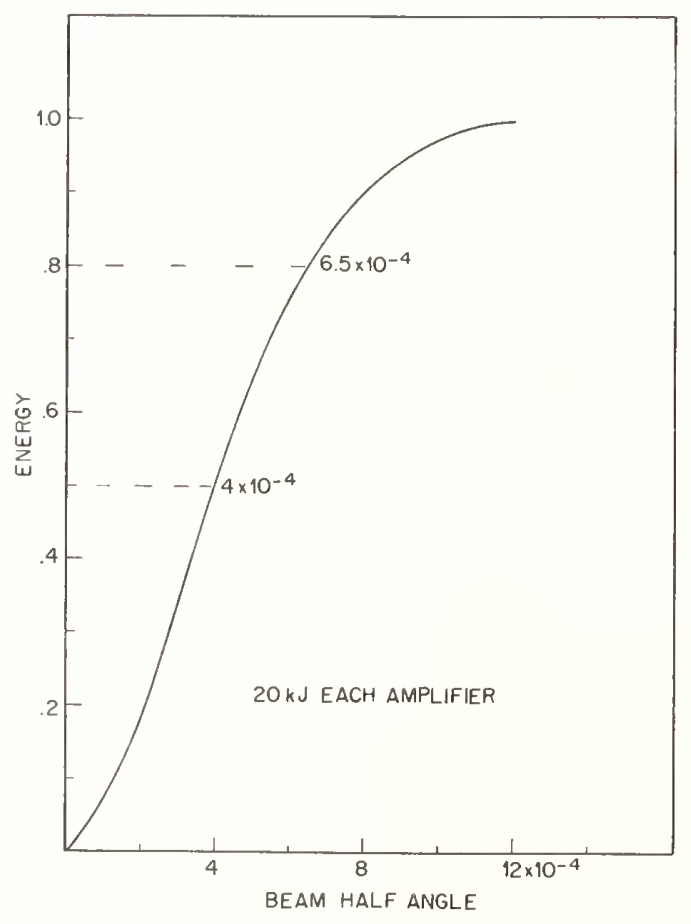

Fig. 7 Far-field energy distribution of entire system with $750 \mu$ sec pump pulse (20 kJ input).

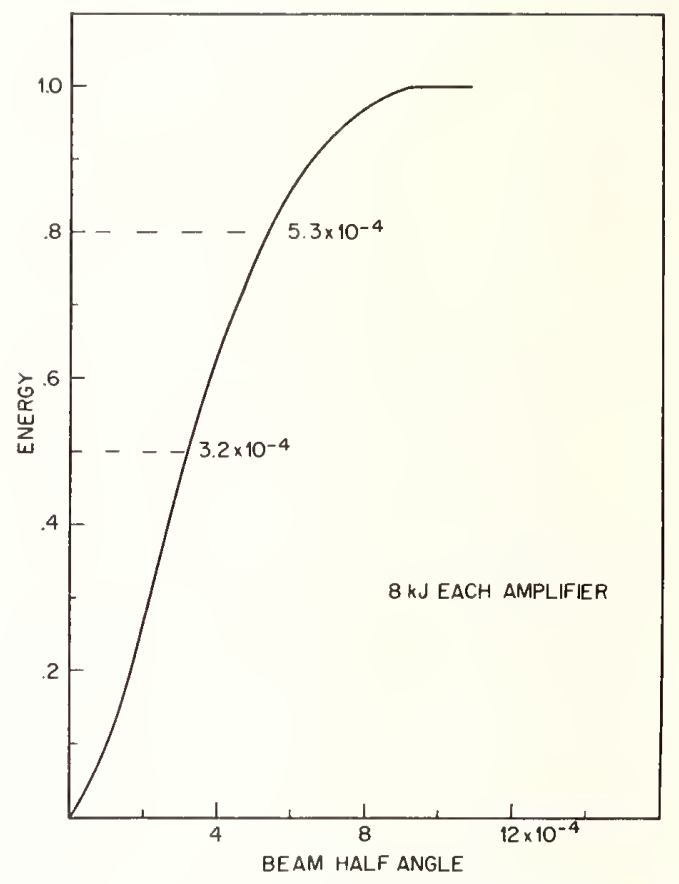

Fig. 6 Far-field energy distribution of entire system with $480 \mu \mathrm{sec}$ pump pulse to final amplifier ( $8 \mathrm{~kJ}$ input).

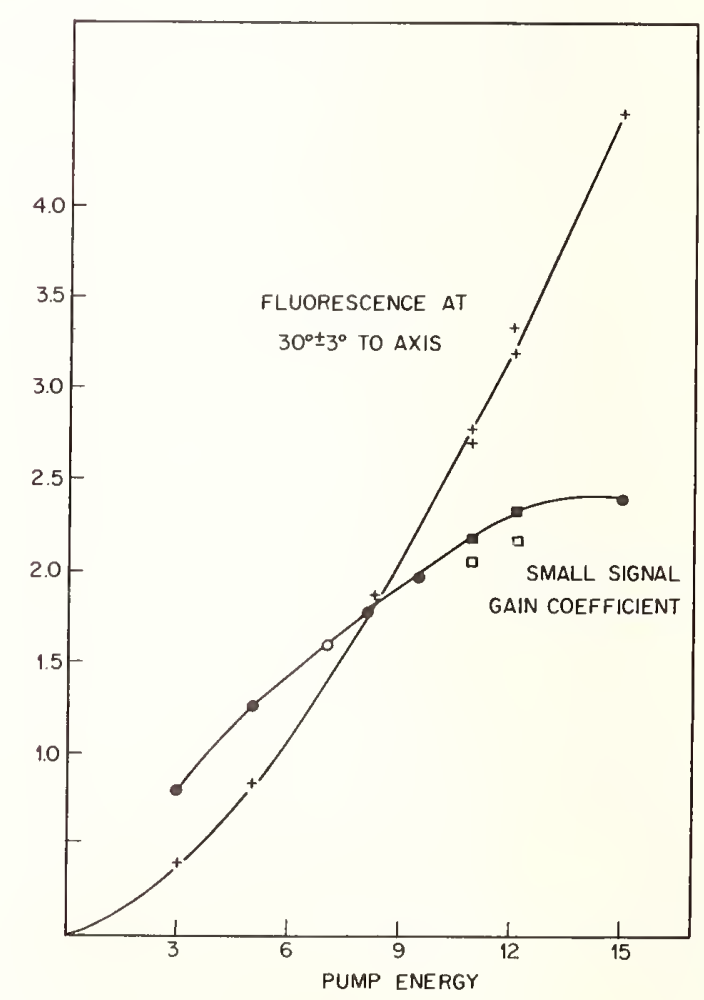

Fig. $8 \quad$ Sma11 signal gains coefficient vs input energy for third amplifier. The fluroescence intensity at $30^{\circ}$ to the optical axis is also shown. 


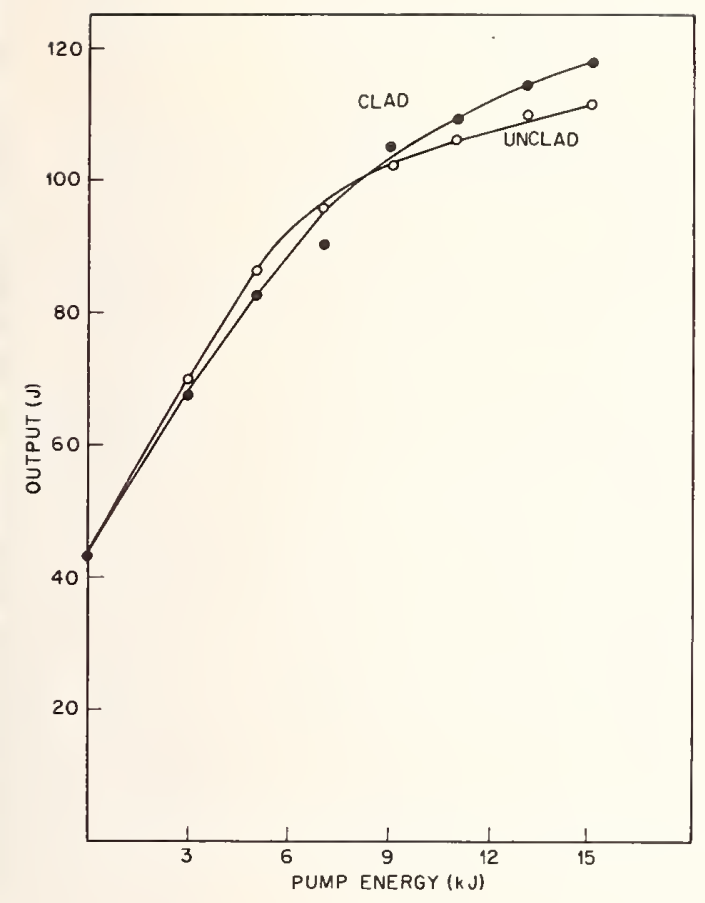

Fig. 9 Total output vs pump energy to final amplifier with and without cladding solution. Beam area was $7.0 \mathrm{~cm}^{2}$ and input pulse was 57 joules in 35 nanoseconds.

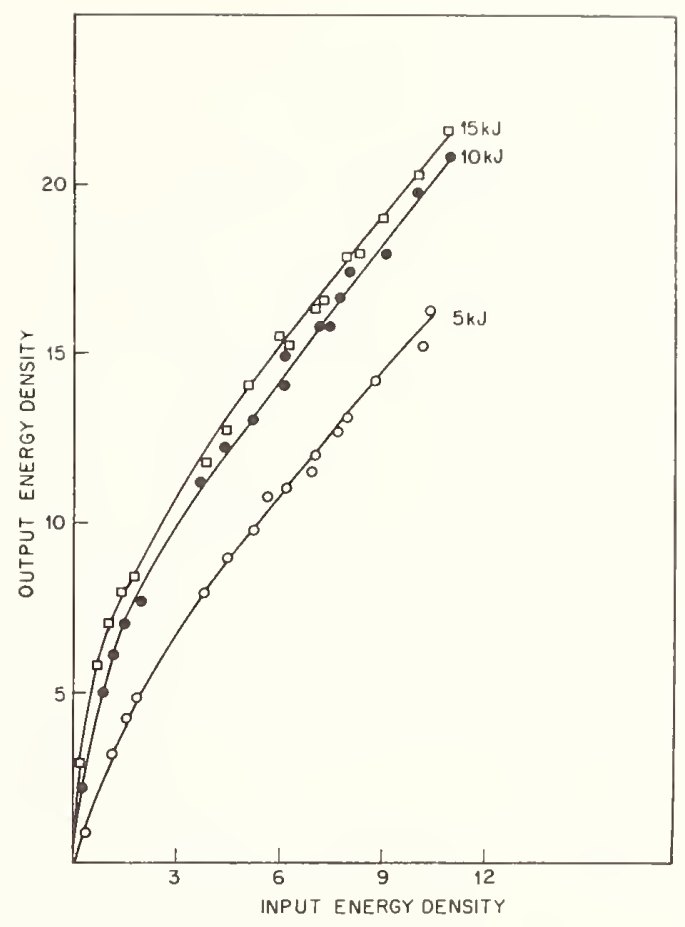

Fig. 10 Laser input vs laser output for third amplifier at 5, 10, and $15 \mathrm{~kJ}$ pump inputs.

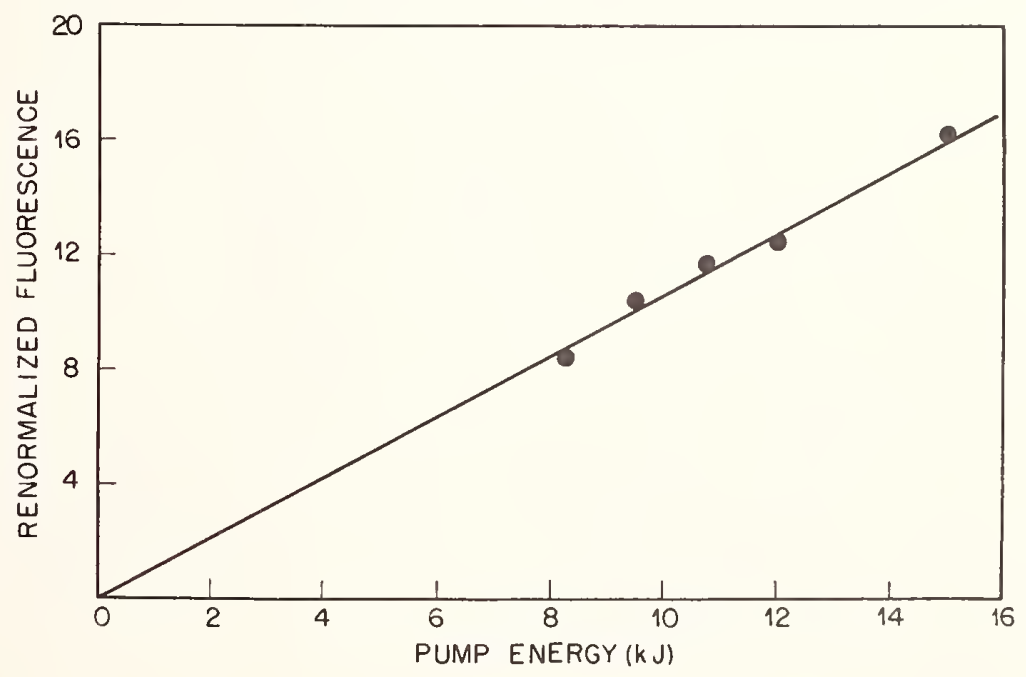

Fig. 11 Fluroescence data of Fig. 9 renormalized using small signal gain coefficients and $\ell$ eff $\sim$ $11 \mathrm{~cm}$. 


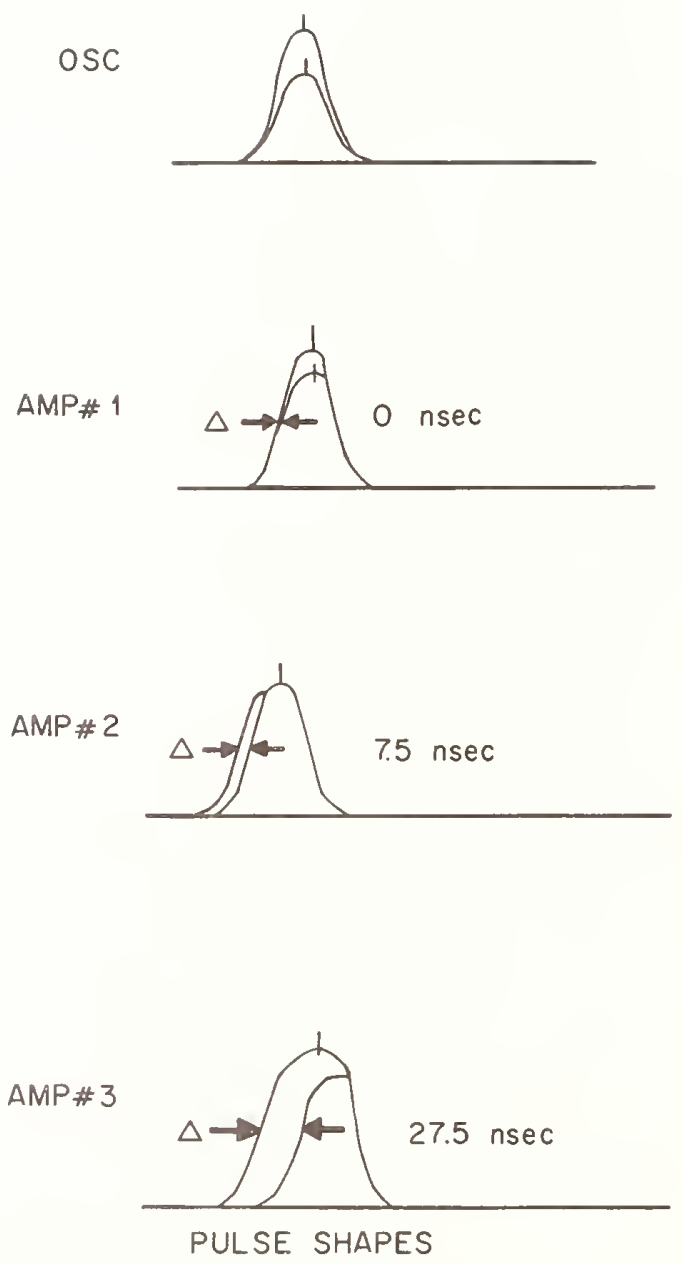

Fig. 12 Oscillator pulse shape and amplified pulse shape at various places in amplifier train. The pulse is not appreciably broadened in the first amplifier, is broadened $20 \%$ in the second amplifier and $\sim 50 \%$ in the third amplifier.

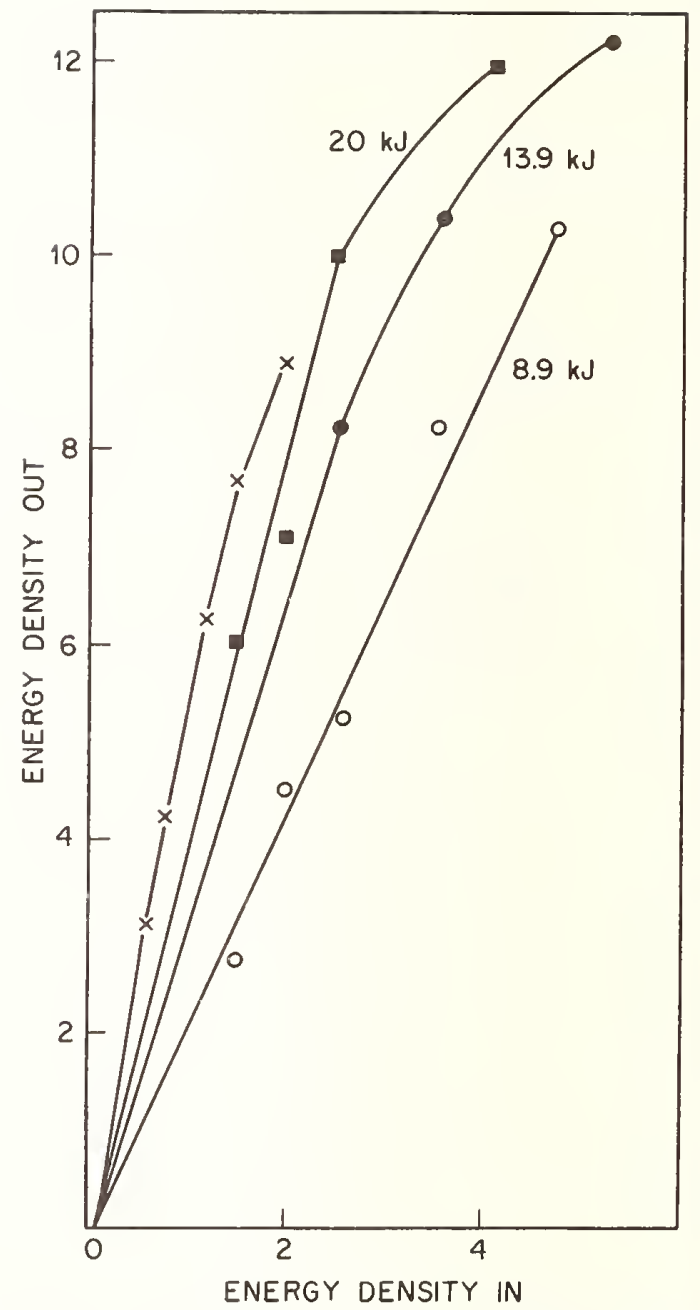

Fig. 13 Laser input vs laser output for AO 2378 laser glass at pump energies of $8.8,13.8$, and $20.0 \mathrm{~kJ}$ with and without ultraviolet filtering. 
The focal beam expanders considerably reduce the normal oscillator beam divergence of about 3 mrad, correcting for the natural sphericity of the wave front as well as thermal distortion introduced by the amplifier stages. The latter is a function of the repetition rate.

It is not appropriate to make a direct comparison of the performance, e.g. beam divergence, of owens-Illinois and C.G.E. glass in the C.G.E. system, since its mode of operation was optimized for the characteristics of a selected, high quality oscillator rod of C.G.E. glass.

Measurements at AFCRL indicate small angle scattering in owens-Illinois glass in agreement with the value inferred from the NRL measurements.

A diffraction pattern is introduced in the near field of the laser output by the break edge of the rotating TIR prism.

The loss of definition of this diffraction pattern observed using owens-Illinois glass over that exhibited with C.G.E. glass is probably due to passive distortion rather than small angle scattering. This was verified when the test rod was subsequently examined at owens-illinois using an interferometric technique similar to that of Baird (L.R.L.). A full wave of distortion was observed in the tesi rod. This test should be a useful quality control indicator of rod performance, and is now in use at owens-Illinois. Or. Snitzer also pointed out that improved quality control procedures have been instituted at American optical company and that the rod supplied for testing at N.R.L. contained three bubbles less than one mon in diameter, which had no major efject on the performance of that specific glass rod.

Mr. Yclaton felt that the observed pulse broadening was due to the long relaxation time of the lower laser levex, which impeded the efficient extraction of energy at high energy densities 12100 joules $\left./ \mathrm{cm}^{2}\right)$. It was also pointed out that great care had to be exercised to eliminate feedback due to reflecting surfaces. Pulse broadening has been observed at owens-Illinois due to reflection from amplifier rod ends cut as much as 10 degrees ofb axis, as well as from target backscatter. The beedback effect was precluded in the NRL tests by moritoring the oscillator and amplifier output pulses synchronously with two diodes.

The analysis of the pulse broadening carried out at NRL was based solely on the use of the rate equation description of the level poputation changes, and the obsurvad $80 \%$ pulse broadening was attributed to a filling of the lower laser level when the rod was pumped at a high level. Dr. Snitzer pointed out that under these conditions, a full quantum-mechanical density matrix description of the relaxation processes is required, including both quantum-mechanical relaxation (T. ${ }_{2}$ as welt as population changes $(T$,$) .$ 
Experimental Investigation of the

Behavior of Neodymium-Glass Laser Amplifiers

C. R. Jones, P. V. Avizonis, and P. Sivgals

Air Force Weapons Laboratory

Kirtland AFB, N. M. 87117

\begin{abstract}
Anomalies observed in gain measurements on Nd:glass laser amplifiers are reported. The investigation, which was undertaken as part of a high-energy laser program, revealed a significant gain fall-off at high amplifier excitation energies. Efforts to determine the origin of this phenomenon are described. The discrepancy between the observed gain and that predicted theoretically for an Nd:glass laser amplifier is found not to be due to superradiance or flashlamp spectral shift. Furthermore, a pump-energydependent loss process in the glass matrix is shown not to contribute to the gain roll-off. The underlying physical process remains undetermined.
\end{abstract}

Key Words: Amplifier, flashlamp, laser, Nd:glass, optical gain, optically pumped laser

\title{
1. Introduction
}

A segmented-glass laser system similar to that sketched in Fig. 1 is currently under development in this laboratory. The available gain from the optical amplifiers in this high-energy system is obviously of prime importance. Moreover, the capability of predicting the gain over a wide range of flashlamp energies and laser input energies is certainly desirable from a design point of view. These considerations led to a recent investigation into the behavior of Nd:glass amplifiers with emphasis on the comparison of the experimental data with the theoretical predictions.

The expression which describes the spatial evolution of an optical pulse in a four-level laser amplifier is $[1]^{1}$

$$
\frac{d E(x)}{d x}=\xi_{s}\left[1-\exp \left(-\beta_{s} E\right)\right]-\alpha E(x)
$$

where $E(x)=$ pulse energy density in joules $/ \mathrm{cm}^{2}$ at position $\mathrm{x}$ in amplifier,

$\xi_{s}=$ inversion density in joules $/ \mathrm{cm}^{3}$ in the active medium at time of pulse,

$\beta_{S}=$ gain coefficient in $\mathrm{cm}^{2} /$ joules, usually defined at peak of fluorescence curve, and

$\alpha=$ loss coefficient of the laser medium at the operating wavelength.

This expression has been modified from that for the three-level scheme derived in the reference. The application of expression (1) to a particular amplifier is quite straightforward on a desk computer if the relevant parameters are known.

The literature revealed very little in the comparison of the theoretical amplifier equation above with experimental results on Nd:glass material. It seemed worthwhile, then, to experimentally verify the equation above with an amplifier experiment incorporating solid glass rods. The results should be relevant to a segmented laser system with the proper adjustment of the loss coefficient.

\section{Experimental}

The experimental apparatus is presented schematically in Fig. 2. Several Nd:glass rods were used as the amplifier, each being pumped in a flashlamp enclosure as drawn to scale in Fig. 3 . The

1 Numerals in square brackets indicate literature references appearing at the end of this paper. 
reflector surface is polished silver foil and the cladding is deionized water. Each set of two lamps in series is driven by a $300 \mu \mathrm{F}$ capacitor bank through a $100 \mu \mathrm{H}$ air-core inductor, producing a smooth current pulse of about $400 \mu \mathrm{sec}$ duration. The excitation efficiency typically exceeds $1 \%$. The $50-$ nanosecond laser input pulse has an intensity profile of about 6 mm diameter.

\section{Results}

\subsection{Gain Results}

Most of the amplifier measurements entailed monitoring the dependence of amplifier gain on flashlamp energy at a selected laser input energy density. The results of the first measurements, shown in Fig. 4, are for three ED-2 rods of lengths 4, 8, and 12 inches. (A11 rods used in this investigation are 0.75 inch in diameter and are Brewster-Brewster.) The input energy density is in each case 1.25 joule/ $\mathrm{cm}^{2}$ (average). The abscissa units of flashlamp energy have been normalized to the rod pumped volume. The theoretical curve generated by eq. (1) has been fitted to the lower energy data points of each experiment through adjustment of the pumping efficiency. A loss coefficient of $0.005 \mathrm{~cm}^{-1}$ and a gain coefficient of $0.14 \mathrm{~cm}^{2} /$ joule were used. It is apparent that for the shorter two rods there is a growing discrepancy between the theoretical curve and the observed data beyond about 50 joules/cm ${ }^{2}$ energy input. The departure of the experimental curve from the theoretical one does not seem to be dependent upon the laser input to the amplifier: The small-signal gain curve for the 8-inch rod (Fig. 5) departs from theory at about the same point on the flashlamp energy scale. Subsequent sections will explore the physical basis of the observed discrepancy.

\subsection{Fluorescence Results}

The most popular explanations for gain fall-off of this type are superradiance depletion and flashlamp blue shift, both of which diminish the inversion. Hence, a series of fluorescence measurements on the Nd:glass amplifiers was initiated. The experimental arrangement is included in $\mathrm{Fig}$. 2 , which shows the pumped-rod fluorescence at $1.06 \mu$ monitored by a photodiode through a light pipe. The fluorescence is observed, as shown, along the geometrical rod axis and not along the optical axis. Thus, hopefully, spontaneous emission from only a small end-segment of the rod is being detected.

The observations on the 4-inch rod are presented in Fig. 6. The plot of relative fluorescence peak against the pump density is agreeably straight, with an expected leveling at high excitation. The flashlamp emission was found to be an insignificant contributor to these readings. Significant superradiance was checked by observing the fluorescence with and without a $100 \%$ reflecting mirror placed near the opposite (to the photodiode) end of the amplifier rod and normal to its optical axis. There was no effect from the mirror on the signal at any excitation level, although on-axis fluorescence was markedly affected.

The results of the fluorescence measurements on the 8-inch ED-2 rod are presented in Fig. 7 . The straight line is rather boldly drawn through what is more of an S-shaped arrangement of data points, although the sharp break (from gain results on this rod) appears to be real. Again, the 100\% mirror had no effect on the fluorescence signal. A correction of the gain curve for the apparent inversion nonlinearity shown in Fig. 7 still indicates a distinct departure of experimental gain from theoretical gain at the same value of about 50 joules $/ \mathrm{cm}^{3}$ pump density.

Fig. 8 is the graphical fluorescence data on the 12-inch rod (no straight line attempted). Perhaps significantly, the curvature of the fluorescence data increases with pumped length of the rod. Correction of the gain curve of Fig. 4 for this pronounced nonlinearity produces a gain fall-off for the 12inch rod very similar to that of the shorter rods, and, in fact, the departure from theory begins at about the same value of pump energy density.

In order to determine how much of the laser rod contributes to the fluorescence signal for this configuration, the 12-inch rod was placed in the pumping enclosure of the 8-inch rod. By varying the distance the rod protruded from the enclosure and monitoring the spontaneous emission at each distance, an indication of the contributing volume was obtained. The results for this measurement are presented in Fig. 9, along with the data for a 12-inch rod with a polished barrel. (Al1 rods in other parts of the experiment have roughened barrels). The smooth rod clearly demonstrates its shortcoming while the roughened-rod data indicates that it exhibits some light-gathering capability. The data, while not completely analyzed, does emphasize the desirability of a $1.06 \mu$ absorbing cladding around a high-gain rod.

\subsection{Other-Glass Results}

A possible pump-dependent absorption process in the base glass, which could certainly explain the observed gain roll off, was checked as follows: An ED-4 (base glass of ED-2) rod of the same dimensions was substituted for the 4-inch ED-2 rod used in the gain measurements. The variation of gain 
(loss) with excitation was observed under the same conditions as before. To within experimental error (5\%) no pump-dependent absorption could be detected over the same energy range covered in the ED-2 amplifier experiment.

That the apparent gain anomaly is confined to Owens-Illinois glass was checked by performing the gain measurements on a 3\% doped American-0ptical laser rod and a French glass laser rod borrowed from Hadron, Inc. These gain results, plotted in Figs. 10 and 11, also show the severe gain fall off observed above.

\subsection{Flashlamp Effects}

The blue shift of the flashlamp spectral output with increasing current density is certainly one of the foremost of the possible explanations for the observed gain fold-over. The first effort in attempting to isolate this phenomenon, as it applies to the gain results, involved measuring the gain while using flashlamps of different bore. Fig. 12 shows the data for the gain of the short ED-2 rod using three types of flashlamps with the same driving circuitry. The current waveforms through the different lamps were observed to be essentially identical, which means that only the current density changed when the lamps were changed. Quite apparent is the greater pumping efficiency of the smallerbore $\operatorname{lamp}^{2}$, although the important feature of this data is that the departure of the observed gain from theory occurs at about the same flashlamp input for all three lamps, despite the difference in current densities. Not surprisingly, however, the 7-mm flashlamp curve seems to be folding over faster at energy levels above 100 joules $/ \mathrm{cm}^{3}$. Above this excitation peak, current densities exceed 5,000 A/cm ${ }^{2}$ in the small-bore lamp.

\subsection{Temperature Dependence of Gain}

The high-average-power requirement on the laser system under development dictates that thermal effects will be of considerable importance in the design of the device. Although the segmented-rod configuration tends to alleviate many of the thermal problems, the interior of each disc will, nevertheless, be at an elevated temperature that will be a function of the operating power. The increased temperature of the laser material could critically affect the gain realized with a high-average-power amplifier. An experiment was recently begun which will determine the variation in gain of an Nd:glass rod with temperature.

The first results are shown in Fig. 13, in which the gain as a function of flashlamp energy is presented for two rod temperatures $-5^{\circ} \mathrm{C}$ and $88^{\circ} \mathrm{C}$. The temperature range is being extended upward to include the anticipated temperatures of interest in segmented-glass-laser operation, since the foregoing data indicates a significantly reduced gain at elevated glass temperatures.

\subsection{Nd:glass Amplifier with Nd:YAG Input}

As Fig. 1 of this report indicates, the high-average-power laser system under development may incorporate an Nd:YAG oscillator-amplifier driver section. It is hoped that the Nd:YAG system output will have an acceptably low beam divergence, although mode-control measures may be required. In any case the amplification of an Nd:YAG pulse by an Nd:glass amplifier is an important consideration, but there appears to be no reported investigation in this area.

The narrow spectral range of the Nd:YAG output, relative to the fluorescence 1inewidth of Nd:glass, will possibly bear upon the utility of an Nd:YAG laser as the driver stage for Nd:glass amplifiers, particularly beyond the small-signal-gain regime. Thus, an experiment to observe the gain characteristic of an Nd:glass amplifier with an Nd:YAG laser input has been set up. The experiment will compare, at equal laser input levels, the gain of the amplifier using a YAG input pulse with that using an input pulse from a glass-laser oscillator.

The first results obtained were for the small-signal-gain regime ( $30 \mathrm{~mJ}^{\mathrm{cm}}{ }^{2}$ input at 1 ess than $10 \mathrm{~dB}$ gain) with the two inputs producing, almost identical gain curves. Preliminary results in the intermediate-signal-gain regime $\left(0.2 \mathrm{~J} / \mathrm{cm}^{2}\right)$ revea1, however, a smaller gain for the Nd:YAG pulse over the full amplifier excitation range. This data is shown in Fig. 14.

\section{Discussion}

\subsection{Other Investigations}

The data in Fig. 4 clearly indicates that the disparity between actual amplifier performance and

2 The EG\&G $13 \mathrm{~mm}$ lamp data is not corrected for the unused one-third of its length. This correction reveals that this lamp has, in this situation, a $20 \%$ greater pumping efficiency that the $7 m m$ lamp, which in turn, is $25 \%$ more efficient than the ILC lamp. 
the theoretical predictions can be quite significant. The first literature, of which we are aware, that reports a discrepancy between observed ampliffer gain and that predicted theoretically, for Nd:glass amplifiers, is a paper by Young and Kantorski presented at the 1964 DOD Conference on Laser Technology [2]. More recently this phenomenon has been examined rather extensively by Beck at Owens-Illinois, Inc, [3] and McMahon at NRL [4]. All the above investigators have noted essentially the same effect in the gain behavior, although there seems to be no agreement as to its origin. The data of Young and Kantorksi [2] supports their conclusion that within their operating range the cause of the gain fall off is a decrease of the flashlamp output in the useful pumping regions with increasing energy input. Their observed small-signal gain, $B(\% / \mathrm{cm})$, normalized fluorescence, and lamp emittance in the green and red exhibited identical dependences on the flashlamp input. Beck [3], after noting a gain fall-off in his amplifier data, examined the effects of the flashlamp spectral shift on the excitation efficiency. His analysis, based upon the flashlamp emission spectrum and the absorption curve of the Nd:glass, indicated a gradually decreasing pumping efficiency with increasing energy input to the lamps. However, this decrease was not enough to cause the full gain discrepancy, there apparently being other underlying processes such as superradiance and/or upper-level absorption losses, as suggested by the author. After observing a gain roll-off with increasing pump energy in an 0-I glass amplifier (particularly pronounced when no ultraviolet-absorbing cladding was used), McMahon [4] ran a series of input-output curves on the amplifier rod. This data did not depend upon the excitation efficiency, since the flashlamp energy was held constant, and the output energy monitored as the laser input was varied. By fitting eq (1) to the observed results for a sequence of flashlamp inputs, it was shown that an increasing absorption coefficient, $\alpha$, with pump energy could account for the abserved gain fall-off. It was shown that the proposed increase in a closely followed an observed increase in lamp UV-blue emission with pump energy.

\subsection{Fluorescence}

In section 3.2 results were reported for the fluorescence measurements, which were motivated by the gain discrepancy shown in Fig. 4. Unfortunately, the fluorescence curves become more S-shaped as the rod length increases, casting the long rod spontaneous emission data into doubt. Also, it appears from the data of Fig. 9 that the fluorescence detected with the experimental arrangement used here does not arise only from a small segment of the rod; in fact, perhaps one-half of the energy comes from the geometrically occluded portion of the rod, apparently being light-piped to the detector. This situation is of course undesirable in this type of measurement since it indicates that some superradiance may be affecting the results. Light piping, of course, can seriously degrade the achievable inversion in a laser medium as pointed out by Sooy et al., who observed the marked degradation of achievable gain in a ruby amplifier brought about by substituting a smooth-barrel ruby rod for one with a rough surface. Further analysis is being performed on the fluorescence results, although tentatively it seems that the spontaneous emission signal for low-gain rods (4-inch rod) has a direct correlation with the actual inversion. For higher-gain rods the curvature of the fluorescence curves in Figs. 7 and 8 may indicate a distortion of the data by superradiance. Unfortunately, perhaps, important measurements of laser parameters have been based upon similar observations of rod fluorescence, notably the experiments of Michon [6] and Belan, et al. [7].

\subsection{Base Glass}

McMahon [4] has shown that the gain fall off could be due to an increase in the loss coefficient with flashlamp energy input. The absence of a pump-dependent loss in the 0-I base glass ED-4 strongly indicates that the gain fold-over is not due to an interaction of the host with the pump light. This does not rule out any pump-dependent loss process, however, in that the neodymium ion itself can interact with the lamp radiation with other than a positive effect.

\subsection{Population Inversion}

The fluorescence results, as discussed in section 4.2, indicate that only the short-rod data unambiguously relates to the gain portion of this experiment. The spontaneous emission measurements on the four-inch rod show that the gain fall-off is not a manifestation of a population inversion falloff. McMahon's experiment [4], particularly the input-output amplifier data at constant flashlamp energy, supports this conclusion. In his experiment each family of data points (output energy density versus input energy density) was fitted with a theoretical curve by adjustment of the parameters $\beta_{\mathrm{s}}$, $\xi_{s}$, and $\alpha$. This was accomplished for several flashlamp energy input values, an increasing $\alpha$ with pump energy affording a proper fit of the theory to the data. However, the stored energy, $\xi$, was found to be almost linear with flashlamp energy over the full range of population inversion. Similarly, Beck's experiment [3] showed that the inversion should fall off slightly with increased pump energy over this range, not nearly enough to account for the observed gain fall off. ${ }^{3}$

3 The amplifier data of both Beck and McMahou encompass an inversion range very close to that of this experiment. Beck, however, noted a larger decrease in excitation efficiency over his energy range, which can be explained by the fact that his pumping efficiency was about one-half that in McMahon's experiment. The doubled flashlamp energy range undoubtedly caused additional spectral shift. 


\subsection{Flashlamp Effects}

The shift of the spectral output of the flashlamps must, at some point, produce a decrease in the pumping efficiency of the laser system, which will in turn lead to a gain fold-over. Apparently, the experiment of Young and Kantorski was performed over an energy range in which the lamp blue-shift was the dominant mechanism behind the observed gain suppression. Their energy input range, corrected for current pulse length, exceeded that of this experiment by a factor of about four.

The effective color temperature of flashlamps is a strong function of the current density, as determined by Goncz and Newel1 [8]. Their results for FX-47A-7.5 lamps are reproduced in Fig. 15 for two current densities. The large blue-shift is evident, and in fact, the lamp radiant output in the spectral region between $0.6 \mu$ and $0.9 \mu$ even appears to decrease with current density.

Within the operating range of this experiment the shift in spectral output of the flashlamps has little effect on the amplifier gain. Were the gain measurements performed over a region of significant lamp blue-shift, the current density through the flashlamps would have pronounced effect on the gain discrepancy. However, as Fig. 12 illustrates, the gain fall-off for the 4-inch amplifier rod is not noticeably altered by a change in lamp current density of a factor of 3.5 . Aside from efficiency, the only apparent change in gain characteristic incurred by replacing the large bore lamps with the smallbore lamps is an additional flattening of the gain curve at the top end. In this region the $7000 \mathrm{~A} / \mathrm{cm}{ }^{2}$ peak current density in the small lamp probably leads to a substantially reduced excitation efficiency.

\section{Conclusions}

The results indicate that the theoretical gain equation in its present form does not adequately predict the gain behavior of an Nd:glass amplifier. The discrepancy between the observed gain and the theoretical gain was found to exist in several types of laser glass and over a range of laser input pulse energies from small-signal through 2.5 joules/ $\mathrm{cm}^{2}$.

Fluorescence data on a short, low-gain amplifier rod, indicates that the gain discrepancy was not due to an inversion-limiting process over the operating range of this study. The negligible effect of fluorescence being reflected back through the amplifier rod helped substantiate the conclusion that superradiance depletion was not significant in this experiment. Furthermore, by employing lamps of different bore, it was observed that a marked change in current density through the flashlamps at the same lamp input energy had no effect on the gain discrepancy. This suggests that the spectral shift of the flashlamp radiant output with changing current density did not noticeably contribute to the observed gain suppression. This flashlamp blue-shift is, however, expected to affect the gain at greater lamp inputs than those of this experiment. The recent studies by other investigators support these conclusions.

By placing a sample of base glass (no Nd doping) in the test amplifier fixture, it was shown that no measurable pump-dependent loss process in the host matrix was responsible for the gain fall-off.

The measurements on the off-axis fluorescence from amplifier rods of different lengths indicate that the observed signal may not be proportional to the population inversion except in the case of short, low-gain rods. This observation is substantiated by the measurements of the fluorescence signal from a pumped rod as a function of its protrusion from the lamp enclosure, with the excited length held constant. Even for a roughened rod, the entire rod volume was found to contribute significantly to the detected signal, although only a few centimeters were geometrically allowed by the position of the detector. The increased probability of superradiance reaching the detector suggests that the results of previously reported experiments based upon fluorescence observations may be in error.

Finally, the results presented herein suggest that an anomaly in the gain behavior of Nd:glass laser amplifiers, which is not explicable by the usual explanations of superradiance and flashlamp blue-shift, does exist. The origin of the discrepancy has not been determined.

\section{References}

[1] Avizonis, P.V. and Grotbeck, R. L., J. Appl. Phys. 37,687 (1966).

[2] Young, C. G. and Kantorski, J.W., "Optica1 Gain and Inversion in Neodymium-glass Lasers," presented at the First DOD Conference on Laser Technology, 1964.

[3] Beck, R.W., "Damage Threshold Studies of Glass Laser Materials," Owens-Illinois, Inc., Semiannual Technical Report under ARPA Contract Number DAHC15-69-C-0303, January, 1970.
[4] McMahon, J.M., "Studies on 32mm OwensIllinois Amplifier Rod," Naval Research Laboratories, Interim Report under ARPA Order 660, March, 1969.

[5] Sooy, W.R., Cougleton, R.S., Dobratz, B.E., and $\mathrm{Ng}$, W.K., Quantum Electronics III, (Columbia Univ. Press, N.Y., 1964) P. 1103.

[6] Michon, M., JQE QE-2, 612 (1966). 
[7] Belan, V. R., Grigoryants, V. V., and Zhabotinski, M. E., Opto-Electronics 1, 3335 (1969).
[8] Goncz, J, H. and Newell, Pe. B., J. Opt. Soc. Am. 56, 87 (1966).

YAG OSCILLATOR

AMP. COMBINATION

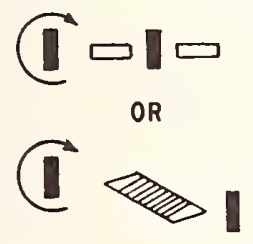

SMALL SEGMENTED GLASS OSCILLATOR
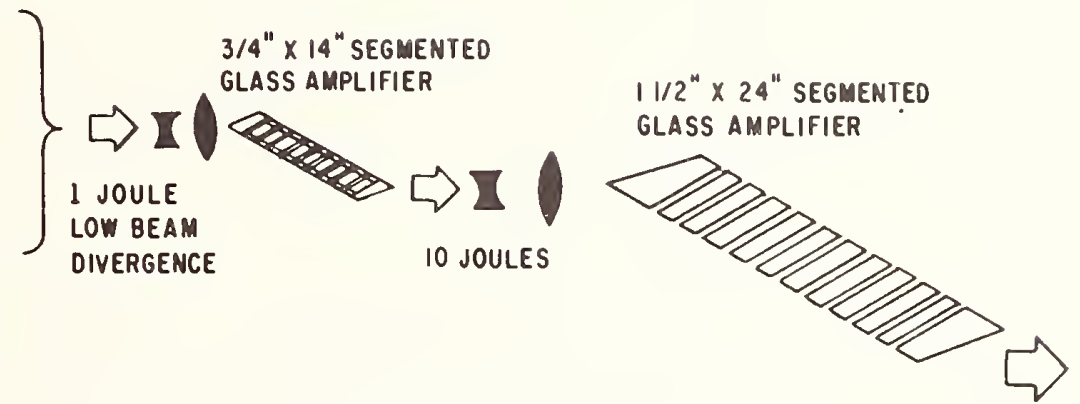

50 JOULES

Fig. 1 Basic components for high-radiance, repetitively pulsed laser system now under development at AFWL.

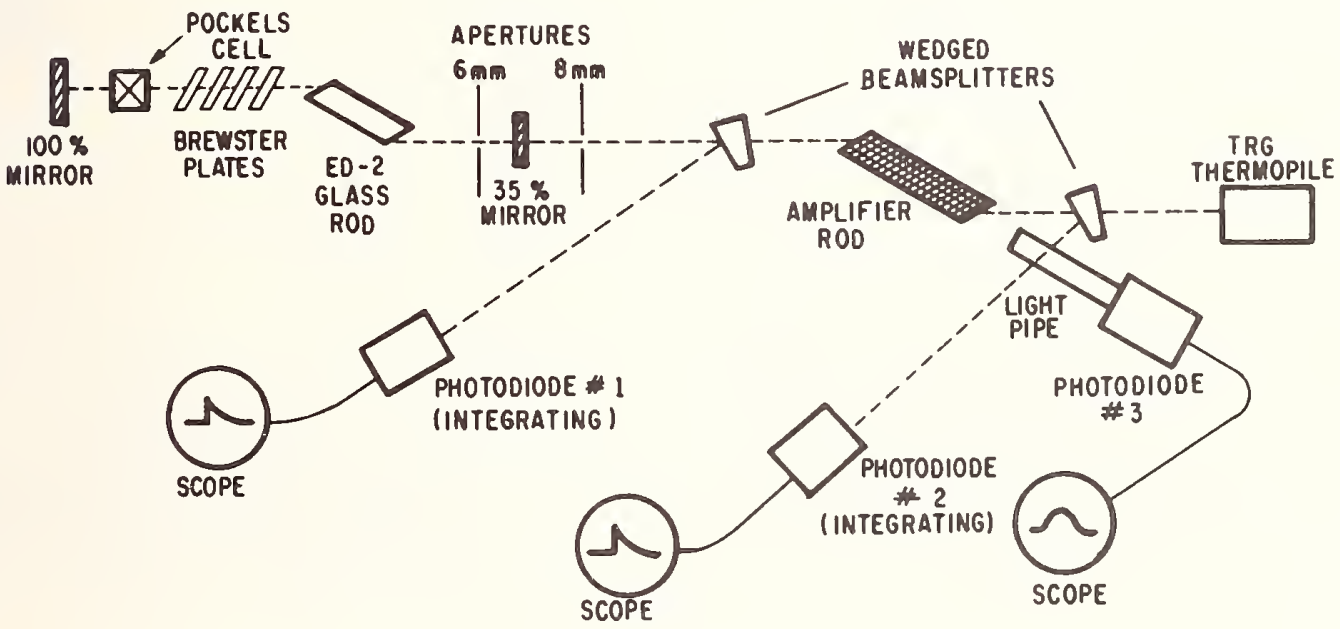

Fig. 2 Experimental set-up for investigation of Nd: glass laser amplifier behavior.

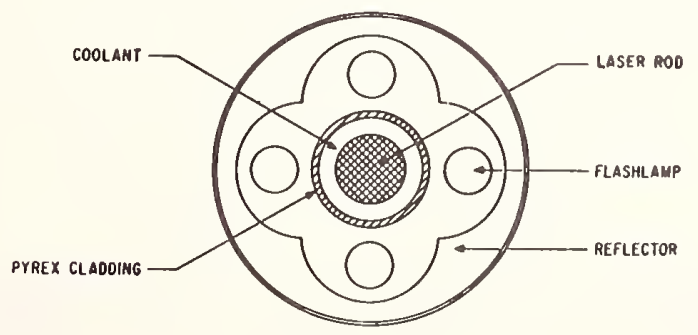

Fig. 3 Cross-section of flashlamp assembly used in amplifier investigation. The sketch is scale for a $0.75-i n c h$ diameter laser rod. The coolant is typically water and the reflector surface is polished silver foil. 


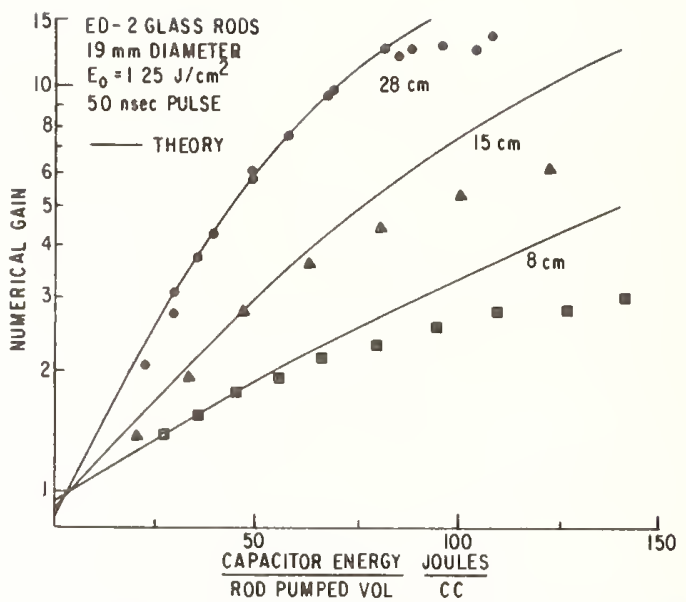

Fig. 4 Numerical gain as a function of input energy for three amplifier rod lengths. The theoretical fit is accomplished for the low-energy data points assuming $\alpha=0.005 \mathrm{~cm}^{-1}$ and $B=0.14 \mathrm{~cm}^{2} /$ joule.

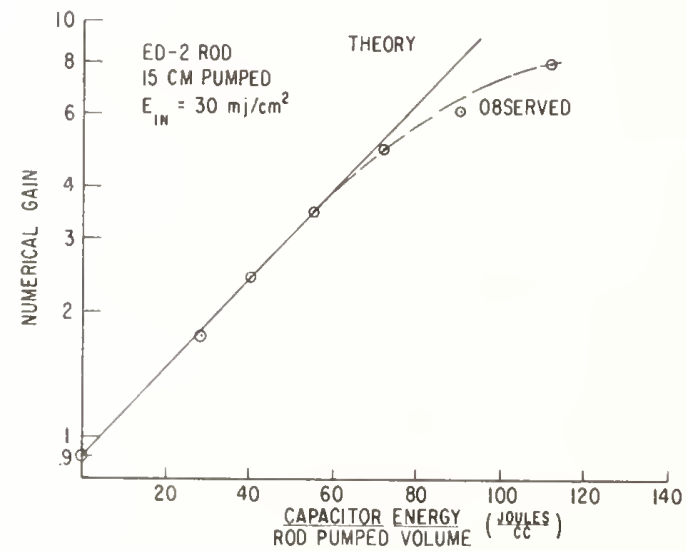

F1g. 5 Small-signal gain has a function of flashlamp energy input for the 8-inch rod.

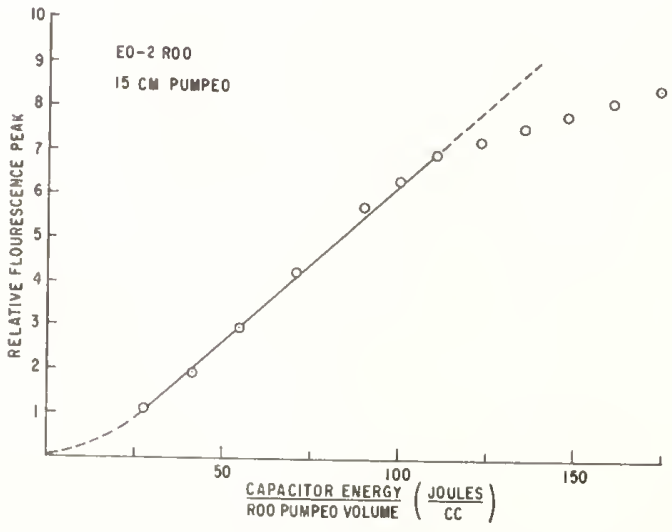

Fig. 7 Peak of fluorescence waveform as a function of flashlamp energy input for the 8-inch rod.

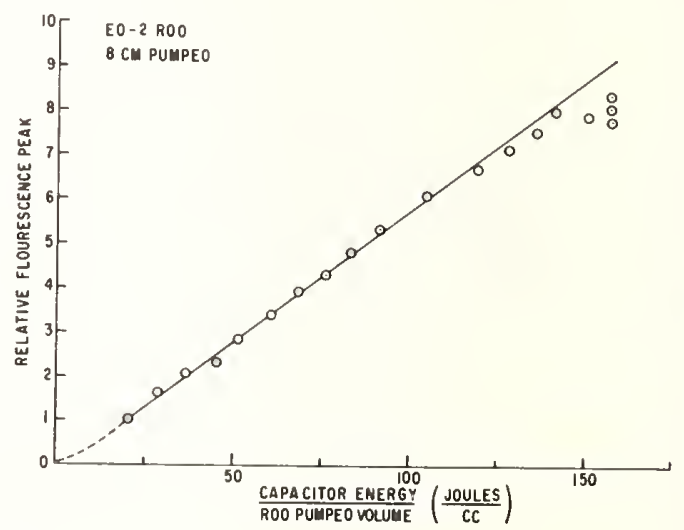

Fig. 6 Peak of fluorescence waveform as a function of flashlamp energy input. The detector is oriented about $35^{\circ}$

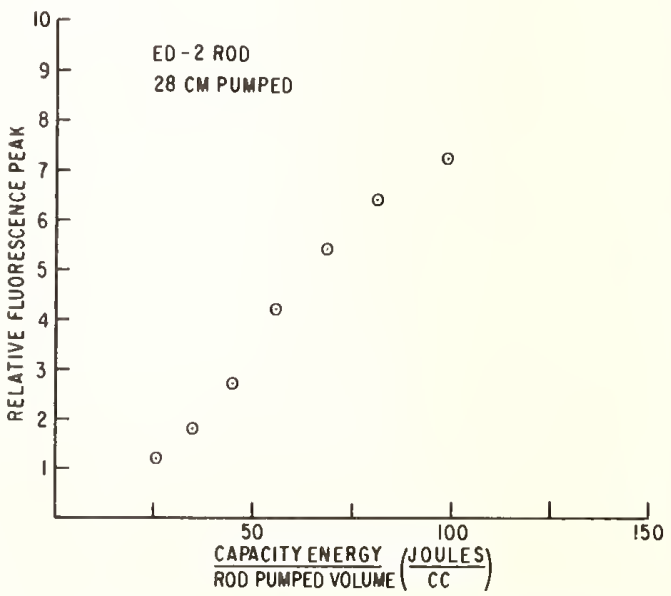

Fig. 8 Peak of fluorescence waveform as a function of flashlamp energy input for the 12-inch rod. 


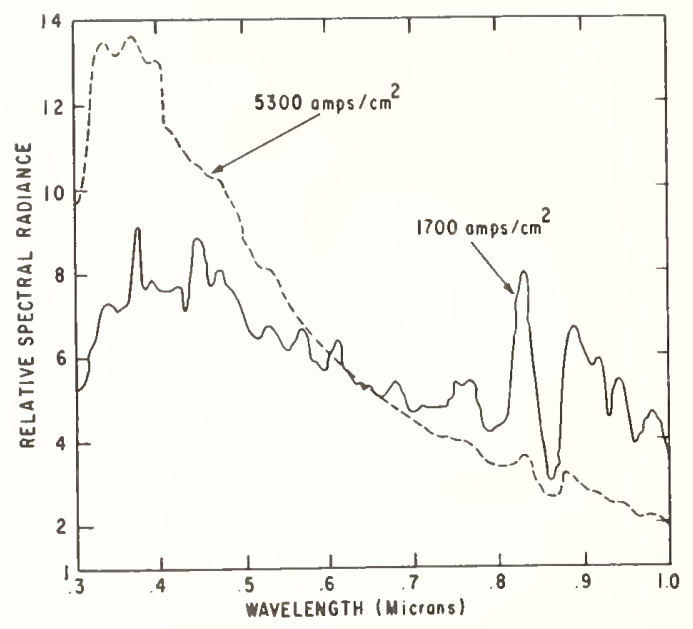

Fig. 15 Flashlamp spectral output for two current densities. After Goncz and Newel1. [8]

\section{COMMENTS ON PAPER BY CAPT C.R. JONES}

It was proposed that a better indication of the inversion level could be obtained by monitoring the $1.35 \mathrm{um}$ radiation, rather than the $1.06 \mathrm{\mu m}$. In measuring the latter, one is troubled by superradiance. An attempt had been made to monitor the $0.88 \mathrm{\mu m}$ and $0.92 \mathrm{\mu m}$ radiation, but the signal in these cases was greatly reduced by self-absorption. The ED-4 glass used in these tests was similar in all respects to ED-2, including cerium, except for the absence of the Nd dopant.

It was pointed out that the $10 \%$ and $15 \%$ reduction in small signal gain observed when a YAG oscillator was employed to drive a glass amplifier over that seen with a glass oscillator could be due to two effects, the spectral mismatch 1ED-2 glass operating at 1.062 $\mathrm{mm}$, Nd: YAG operating at $1.0648 \mathrm{~mm})$, and saturation. The latter was not evident in these experiments. The effect on measured gain due to spectral mi:match has been verified both at American Optical, with A.0. glass, and in the Ph.D. thesis of Duston at RPI, using both American Optical and ED-2 glass.

Although not evaluated in this study it was pointed out that the YAG oscillator output is linearly polarized and that the effective gain in the glass amplifier may be reduced by birefringence induced in the amplifier rod by thermal effects resulting from the intense pumping, as well as residual strains. 
Filamentary Damage in Glasses ${ }^{1}$

\section{J. Davit}

Centre de Recherches de la Compagnie Generale d'Electricite, Division des Applications Optiques, Marcoussis (91) - FRANCE

The diameter of the self focusing filament in glass is measured by different means, all of which give self-consistent results.

The filamentary damage threshold is related to a self-focusing length. The experiments show that the filamentary damage threshold varies with the length of the glass as indicated by the theory of self-focusing only for lengths of glass that are smaller than the self-focusing length.

Key Words: Damage threshold, filaments, self-focusing.

\section{Introduction}

Small scale filaments appear in glass subjected to high power, Q switched laser beams (1). Actually this type of damage limits the breakdown threshold of the glass when the pulse length is 2 ns (2). A typical filament is shown in fig. 1. We have previously described (2) such a filament as composed of a bright filament of a diameter $\mathrm{d} \simeq 0.6 \mathrm{\mu m}$. embedded in what appears to be a hollow cylinder of a diameter $\mathrm{D} \simeq 2 \mu \mathrm{m}$.

We show here that the characteristic diameter of the filament is neither d nor D but a third diameter $s \simeq 0.9 \mu \mathrm{m}$.

The filament in fig. I has been observed with a Reichert microscope by using transmitted and reflected light for illumination. Figure 2 shows the same filament when only the transmitted light is utilized. Then the diameter $s \simeq 0.9 \mu$ appears more clearly than in $\mathrm{fig} . \mathrm{I}$ and we note that the diameter $\mathrm{d}$ is now reduced to $0.3 \mathrm{\mu m}$.

In order to show that the characteristic diameter of the filament is $s \simeq 0.9 \mu \mathrm{m}$, we took a picture in a plane situated at a distance $\mathrm{b}=10 \mathrm{\mu m}$. behind the filament. The point source was at a distance $a=15 \mathrm{~mm}$ in front of the filament. We obtain then the diffraction pattern of an opaque strip with a Fresnel variable difference (3) $\Delta \mathrm{v}=0.5(\mathrm{fig} \cdot 3)$.

The size s of the strip is readily obtained by the equation:

$$
s=\Delta v \sqrt{\frac{a b \lambda}{2(a+b)}}
$$

Because $a \gg b$, the diffraction pattern does not depend on $a$, and this was verified. Now by substituting the values $\Delta v=0.5, \mathrm{~b}=10 \mu \mathrm{m}$ and $\lambda=0.56 \mu \mathrm{m}$, the effective wavelength of white light, we obtain a value $s=84$ um (4).

${ }^{1}$ Work supported by Direction des Recherches et Moyens d'Essais. 
The size of the strip may also be obtained by using Cornu's spiral. The first black fringe is such that $v=2.1$. This corresponds to a distance of $3.85 \mathrm{~mm}$ measured on fig. 3. Thus the size of the strip is $s=0.92 \mathrm{~mm}$.

Finally, we took a photograph of the filament with a polarization interferometer (5) adapted on the Reichert microscope. Then the filament is only characterized by the diameter $s$ which is the true diameter of the filament since the index of refractio inside is different from the outside. Furthermore it is seen that in the part of the photograph on the right side of the line xy the filament has almost the same diameter which does not appear on fig.l.

\section{Variation of the Filamentary Damage Threshold with the Glass Length}

The experiments have been conducted with two laser glasses, A and C, for an excitation wavelength of $1.06 \mathrm{~mm}$ and a pulse length of $2 \mathrm{~ns}$.

These glasses have been selected for the experiments from the many laser glasses we have tested because of the good reproducibility of the results obtained from one sample to another and because of the facility in testing the self-focusing theory with them. Laser glass A is a LG 55 SCHOTT glass. Laser glass C is a MG 915 SOVIREL glass manufactured in a continuous unit process. The peculiarity of this glass is that the proportion of neodymium has been continuously varied during the process in such a way that at the beginning of the process we had:

$\mathrm{La}^{2} \mathrm{O}^{3}: 3 \%-\mathrm{Nd}^{2} \mathrm{O}^{3}: 0 \%$ and at the end of the process

$\mathrm{La}^{2} \mathrm{O}^{3}: 0 \%-\mathrm{Nd}^{2} \mathrm{O}^{3}: 3 \%$.

The results which we shall describe now are the same for the laser glass $C$ with and without Nd. This is important because we shall see that the coefficient $n_{2}$ in the non linear addition to the refractive index must be measured with the laser glass $\mathrm{C}$ without neodymium.

In order to clarify the results we have defined three thresholds:

- The input surface breakdown threshold, defined as the minimum energy density at which a visible breakdown occurs on the input surface of the glass.

- The filamentary damage threshold, defined as the minimum energy density at which a filament begins to appear in the bulk of the glass.

- The output surface breakdown threshold, defined as the minimum energy density at which a visible breakdown occurs on the output surface of the glass.

\section{Results for Laser Glass C}

- When the length of the glass $C$ is greater than $25 \mathrm{~mm}$, the output surface breakdown threshold is $9 \mathrm{~J} / \mathrm{cm}$ in $2 \mathrm{~ns}$. It is constant with the length of the glass and equal to the filamentary damage threshold.

- When the length of the glass $C$ is $20 \mathrm{~mm}$, the output surface breakdown threshold is $11 \mathrm{~J} / \mathrm{cm}^{2}$ in $2 \mathrm{~ns}$. It is still equal to the filamentary damage threshold.

- When the length of the glass $\mathrm{C}$ is below $15 \mathrm{~mm}$, the output surface breakdown threshold is equal to the input surface breakdown threshold that is $14 \mathrm{~J} / \mathrm{cm}^{2}$ in $2 \mathrm{~ns}$, but the filamentary damage threshold is higher.

When the length of the glass $\mathrm{C}$ is $4 \mathrm{~mm}$, the filamentary damage threshold is about $55 \mathrm{~J} / \mathrm{cm}^{2}$ in $2 \mathrm{~ns}$. 
4. Results for Laser Glass A

- When the length of the glass $A$ is greater than $20 \mathrm{~mm}$, the output surface breakdown threshold is $17 \mathrm{~J} / \mathrm{cm}^{2}$ in $2 \mathrm{~ns}$. It is constant with the length of the glass and equal to the filamentary damage threshold.

- When the length of the glass $A$ is below $10 \mathrm{~mm}$ the cutput surface breakdown threshold is equal to the input surface breakdown threshold, that is $21 \mathrm{~J} / \mathrm{cm}^{2}$ in $2 \mathrm{~ns}$, and the filamentary damage threshold is higher.

- When the length of the glass $A$ is $15 \mathrm{~mm}$, the output surface breakdown threshold is either $21 \mathrm{~J} / \mathrm{cm}^{2}$ or $17 \mathrm{~J} / \mathrm{cm}^{2}$. Thus the precision for the measurement of the filamentary damage threshold of laser glass A is not as good as with laser glass C.

When the length of the glass $A$ is $4 \mathrm{~mm}$ the filamentary damage threshold is about $70 \mathrm{~J} / \mathrm{cm}^{2}$ in $2 \mathrm{~ns}$.

\section{Interpretation of the Results by the Self-Focusing Theory}

In order that the experimental results agree with the self-focusing theory, it is important to remember, as was pointed out by Bespalov and Talanov (6), that among the individual inhomogeneous sections in the cross section of the laser beam, there is an optimal transverse spatial scale a opt for which the self-focusing proceeds at the fastest rate.

Now if we consider the stationary aberrationless self-focusing in a medium with $\varepsilon=\varepsilon_{0}+\varepsilon_{2} E^{2}, \varepsilon_{2}>0$ and if we take account of the diffraction, then the selffocusing theory indicates (7) that a beam of diameter 2 a with a plane phase front and parabolic amplitude focuses inself into a point over a length $R_{\mathrm{n} \ell}{ }^{\mathrm{d} 1}{ }^{\ell}$ given by:

$$
\left[\frac{1}{\mathrm{R}_{\mathrm{n} \ell} \mathrm{dif}}\right]^{2}=\frac{1}{\mathrm{R}_{\mathrm{n} \ell}{ }^{2}}-\frac{1}{\mathrm{R}_{\mathrm{d}}^{2}}
$$

where:

$$
R_{\mathrm{nl}}=a \sqrt{\frac{\varepsilon_{0}}{2 \varepsilon_{2} E^{2}}} \text { is the self-focusing length in the geometrical-optics }
$$

approximation $\left(k=\frac{2 \pi}{\lambda} \rightarrow \infty\right)$.

$$
\mathrm{R}_{\mathrm{d}}=\mathrm{k} \frac{\mathrm{a}^{2}}{2} \text { is the diffraction length of the beam. }
$$

The optimal tranverse spatial scale $a_{\text {opt }}$ is determined from the condition $\frac{\partial R_{n l}^{d i f}}{\partial a}=0$
gives: which gives:

$$
a_{\text {opt }}=\frac{2}{k} \quad \sqrt{\frac{\varepsilon_{0}}{\varepsilon_{2} E^{2}}} \quad \text { and } z_{f \min }=k \frac{a_{o p t}^{2}}{2}
$$

where $\mathrm{z}_{\mathrm{f} \text { min }}$ is the minimum self-focusing length. By combining these relations and introducing the refractive index inside the beam $n_{d}=n_{0}+n_{2} E^{2}$ such that $\frac{n_{2}}{n_{0}}=\frac{\varepsilon_{2}}{2 \varepsilon_{0}}$, we obtain:

$$
z_{f \min }=\frac{\lambda_{0}}{2 \pi n_{2} E^{2}}
$$


It is seen that the experimental results obtained with laser glass C agree very well with the preceding equation, since:

$$
25 \mathrm{~mm} \times 9 \mathrm{~J} / \mathrm{cm}^{2} \simeq 20 \mathrm{~mm} \times 11 \mathrm{~J} / \mathrm{cm}^{2} \simeq 4 \mathrm{~mm} \times 55 \mathrm{~J} / \mathrm{cm}^{2}
$$

The precision is not so good with laser glass A, in particular because the input surface breakdown threshold of $21 \mathrm{~J} / \mathrm{cm}^{2}$ in 2 ns is too near the output surface breakdown threshold of $17 \mathrm{~J} / \mathrm{cm}^{2}$ in $2 \mathrm{~ns}$, which renders the measurements difficult.

\section{Measurement of the $\mathrm{n}_{2}$ Coefficient for Laser Glass $\mathrm{C}$}

- All of the details of the experiment which allows us to evaluate this coefficient will be given elsewhere (8). The scheme of this experiment is shown in Fig. 5.

The anisotropy is induced in the glass by the intense light wave (maximum energy density $3 \mathrm{~J} / \mathrm{cm}^{2}$ in $30 \mathrm{~ns}$ at $1.06 \mathrm{~mm}$ ). The $5300 \AA$ radiation blocked in the absence of the anisotropy by the analyzer A is incident on a photomultiplier when the laser pulse renders the glass anisotropic. The filter $F$ passes this radiation.

The kerr constant is calculated from the experimentally determined difference of the refractive index $n_{11}-n_{\perp}=\lambda_{0}{ }^{B}$ opt $E^{2}$ with $\lambda_{0}=5300 \AA$. If we suppose that the contribution to $\mathrm{n}_{2}$ is due to changes in the polarizability of the molecules we have the relation:

$$
n_{2}=\frac{3}{2} \quad B_{\text {opt }} \lambda_{0}
$$

Now because the laser glass has an absorption band at the wavelength of $5300 \AA$. we are obliged to measure the $\mathrm{n}_{2}$ coefficient with the laser glass $\mathrm{C}$ without Na. We have seen previously that the filamentary damage threshold of laser glass C is independant of the proportion of the $\mathrm{Nd}$, that is the $\mathrm{n}_{2}$ coefficient is the same.

The measurement of the kerr constant in the case of laser glass C without Nd is Bopt $=1.1 \times 10^{-8}$ esu.

Therefore we find, by postulating an electronic kerr effect:

$$
\mathrm{n}_{2}=8 \cdot 7 \cdot 10^{-13} \text { esu. }
$$

\section{Calculations Based on Self-Focusing Theory}

- The equation for $Z_{\text {fmin }}$ that we have given above and the experimental results obtained with laser glasses $\mathrm{A}$ and $\mathrm{C}$ allow us to calculate the $\mathrm{n}_{2}$ coefficient for these glasses.

\section{1 Laser Glass C}

If we take $25 \mathrm{~mm}$ as a self focusing length, the filamentary dąmage threshold is $9 \mathrm{~J} / \mathrm{cm}^{2}$ in $2 \mathrm{~ns}\left(\mathrm{E}=5 \mathrm{x} 10^{3} \mathrm{esu}\right)$. By using the relation $\mathrm{z}_{\mathrm{f}}=\frac{\lambda_{\mathrm{O}}}{2 \pi \mathrm{n}_{2} \mathrm{E}^{2}} \mathrm{we} \mathrm{find}$
$\mathrm{n}_{2}=2.7 \mathrm{x} 10^{-13}$ esu. 
There is about a factor 3 with the $\mathrm{n}_{2}$ measured for this glass. We must remember that the measurement of the $\mathrm{n}_{2}$ coefficient was made with a pulse length of $30 \mathrm{~ns}$, while we calculate the $\mathrm{n}_{2}$ from the results obtained with a pulse length of 2 ns. This may explain the observed difference. The critical power is obtained from the relation $P_{\text {crst }}=\frac{\lambda_{0}^{2} C}{32 \pi^{2} n_{2}}$ and we find for
the laser glass $C, P_{c r 4 t}=395 \mathrm{KW}$, of the order of the
critical power obtained from the electrostrictive focusing $(9-10)$.

\subsection{Laser Glass A}

With this laser glass, if we choose $16 \mathrm{~mm}$ as a self-focusing length and $17 \mathrm{~J} / \mathrm{cm}^{2}$ in $2 \mathrm{~ns}\left(\mathrm{E}=6.9 \times 10^{3} \mathrm{esu}\right)$ as a filamentary damage threshold, the same calculation gives: $n_{2}=2.2 \times 10^{-13}$ esu, not much different from laser glass $A$. Therefore, we cannot explain the difference in the filamentary damage thresholds of laser glasses $A$ and $C$ with only the $\mathrm{n}_{2}$ parameter.

In fact it is seen that the principal difference between these glasses is that the maximum of the optimal transverse spatial scale a opt calculated from the selffocusing length is $75 \mathrm{~mm}$ for laser glass $\mathrm{C}$ and $60 \mathrm{~mm}$ for laser glass A.

It is interesting to note that many inhomogeneities of the beam of our laser system have a mean diameter $2 \mathrm{a}$ of about $100 \mathrm{~mm}$.

8. Pulse Length Variation of the Filamentary Damage Threshold in the Range 2 to 4 NS

We have tested the laser glass $\mathrm{C}$ with a pulse length of $4 \mathrm{~ns}$. In this case the output surface breakdown threshold is equal to the filamentary damage threshold, found to be $18 \mathrm{~J} / \mathrm{cm}^{2}$ in $4 \mathrm{~ns}$.

Therefore in the range 2 to $4 \mathrm{~ns}$ the filamentary damage threshold is determined by the power density of the laser beam. We recall (2) that with laser glass A the filamentary damage threshold is much higher than the surface damage threshold, which is another difference between these glasses.

\section{Conclusion}

The experiments we have described show that the filamentary damage is due to the self-focusing of the beam. We think that the electronic kerr effect plays an important role in the contribution to the $\mathrm{n}_{2}$ coefficient. It is not quite clear why two glasses with an $\mathrm{n}_{2}$ of the same order have so different filamentary damage thresholds.

It is possible that the approximations made in the case where we have applied the self-focusing theory are not valid above a certain diameter. For example the beam profile may possibly deviate from parabolic and if this is true this can explain why the experimental results are not fitting the theory for a length of the glass greater than the self-focusing length.

\section{References}

(1) Hercher, M., "Laser induced damage in transparent media", J. Opt. Soc. of America, 54, 563(1964).

(2) Davit, J.., "Damage in laser glass", ASTM - STP 469, 100 (N.B.S. Boulder, Colo, June 1969)
(3) Jenkins, F.A. and White, H.E.. Fundamentals of optics, (McGraw-Hill, Inc., New York, 1950) 2nd ed p.373.

(4) Rigorously, as the index of refraction of the glass is 1.5 , we have $\mathrm{b}=1.5 \times 10 \mu$ and $\lambda=0.56 / 1.5 \mu$ which gives the same result for $\mathrm{s}$. 
(5) Francon, M.. Modern applications of Physical Optics, Interscience Publishers.

(6) Bespalov, V.I. and Talanov, V.I., "Filamentary structure of light beams in non linear liquids". JETPLett $3,307(1966)$.

(7) Akhmanov, S.A., et al, "Self focusing and diffraction of light in a non linear medium", Soviet Physics Uspekhi. Vol 93 p. 609, March-April 1968.
(8) Lacour, B. and Pocholle, J.P., "Optical Kerr constant measurement in Iiquids and glasses", to be published.

(9) Kerr, E.L.. "Laser beam self focusing and glass damage caused by electrostrictively driven acoustic waves", same reference as in (2).

(10) Quelle, F.W., "Self focusing in glass", same reference as in (2).

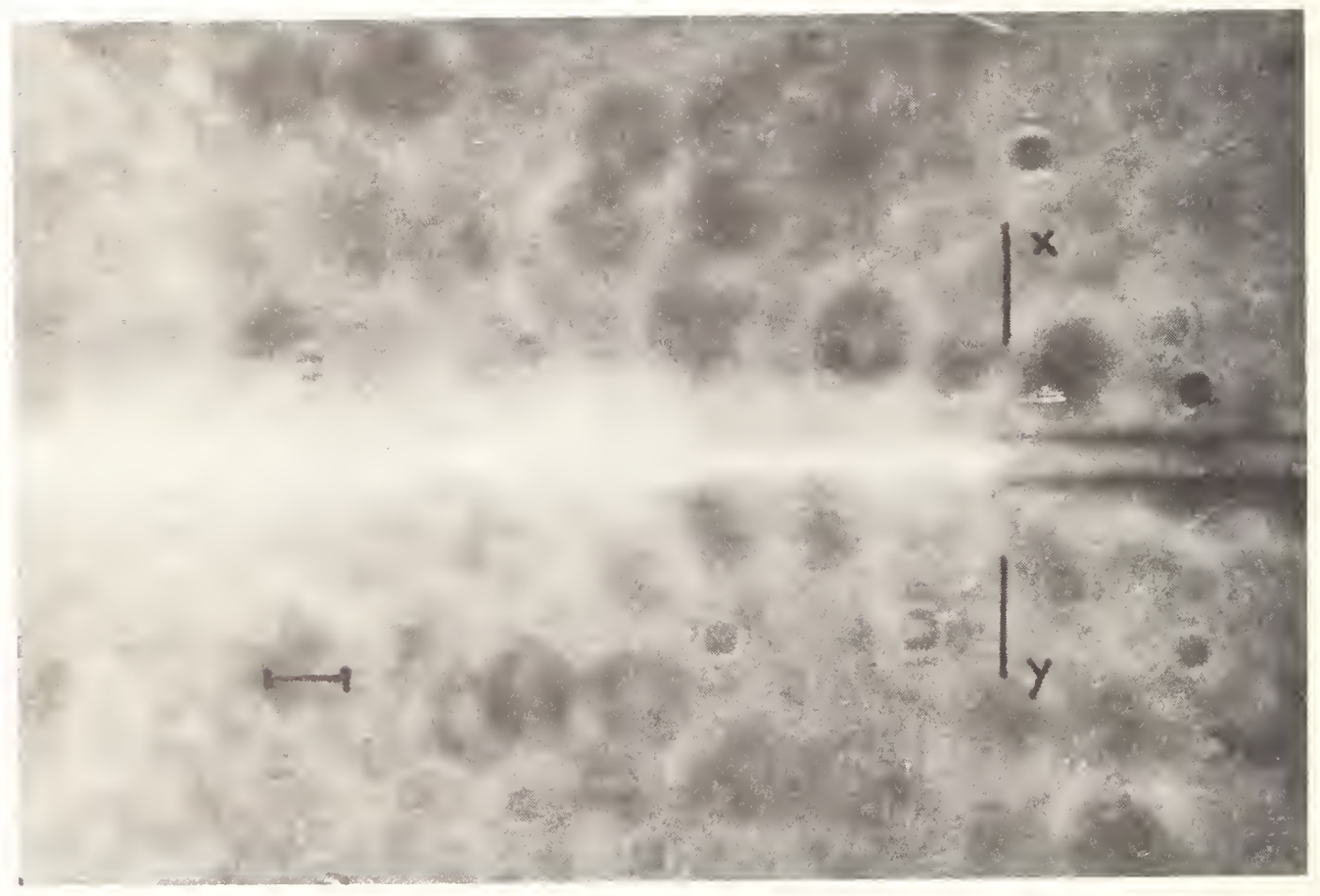

Fig. 1 A typical filament obtained in laser glass with a 2 nsec pulse, observed with a Reichert microscope under both transmitted and reflected illumination. Reference distance $=10 \mu \mathrm{m}$ in each figure. 


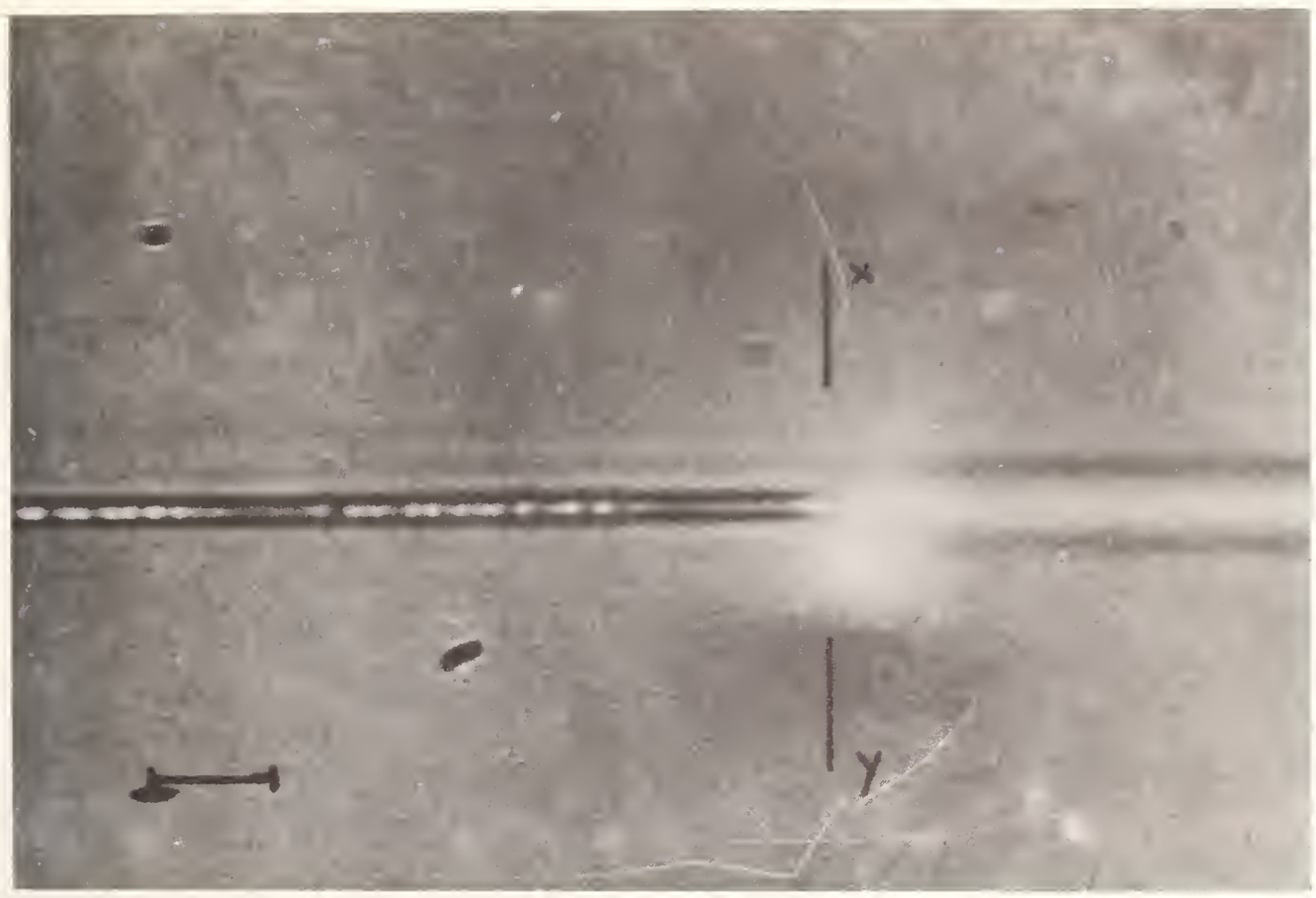

Fig. 2 The same filament observed in transmitted light only.

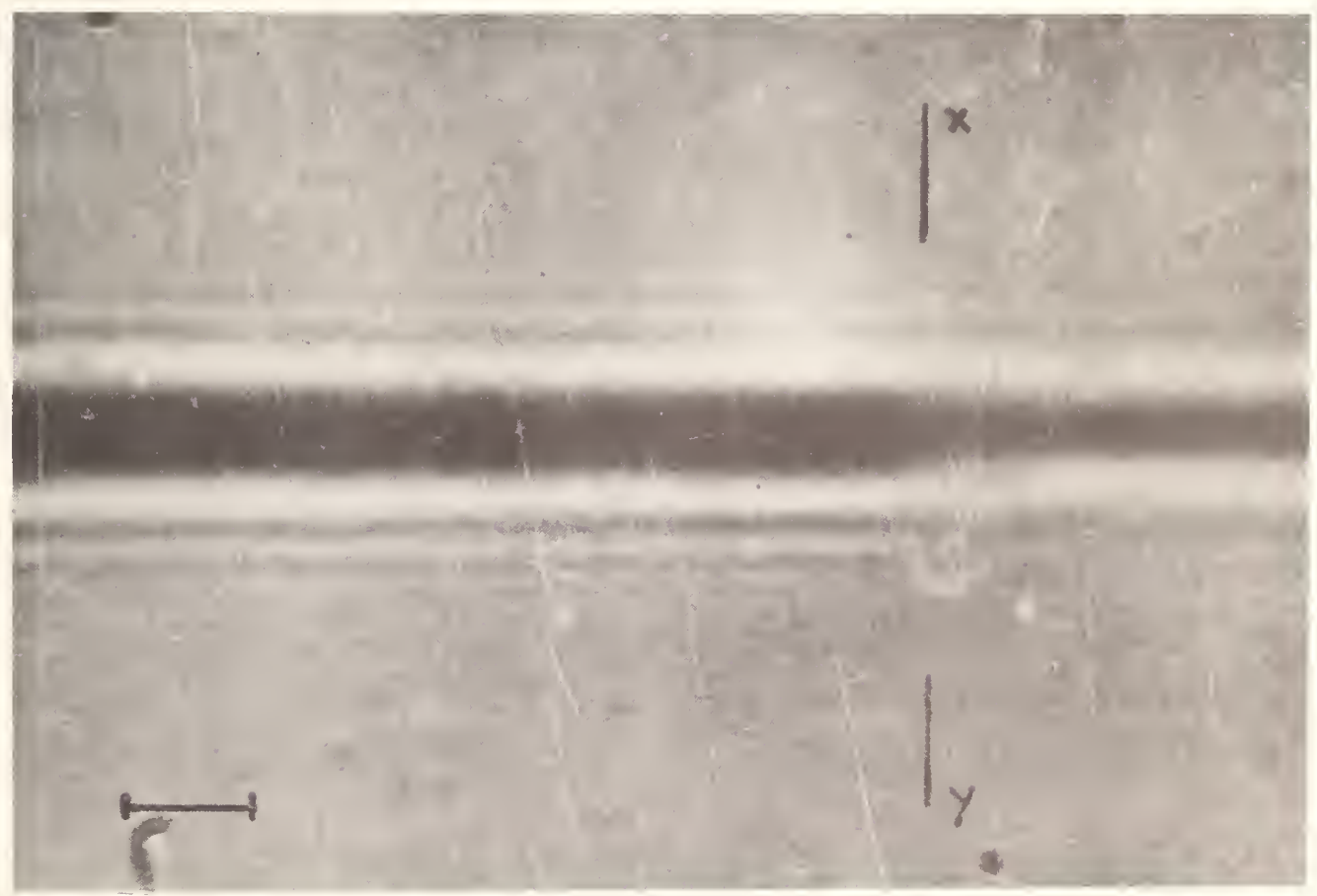

Fig. 3 Image of the filament in a plane $10 \mu \mathrm{m}$ behind the filament. 


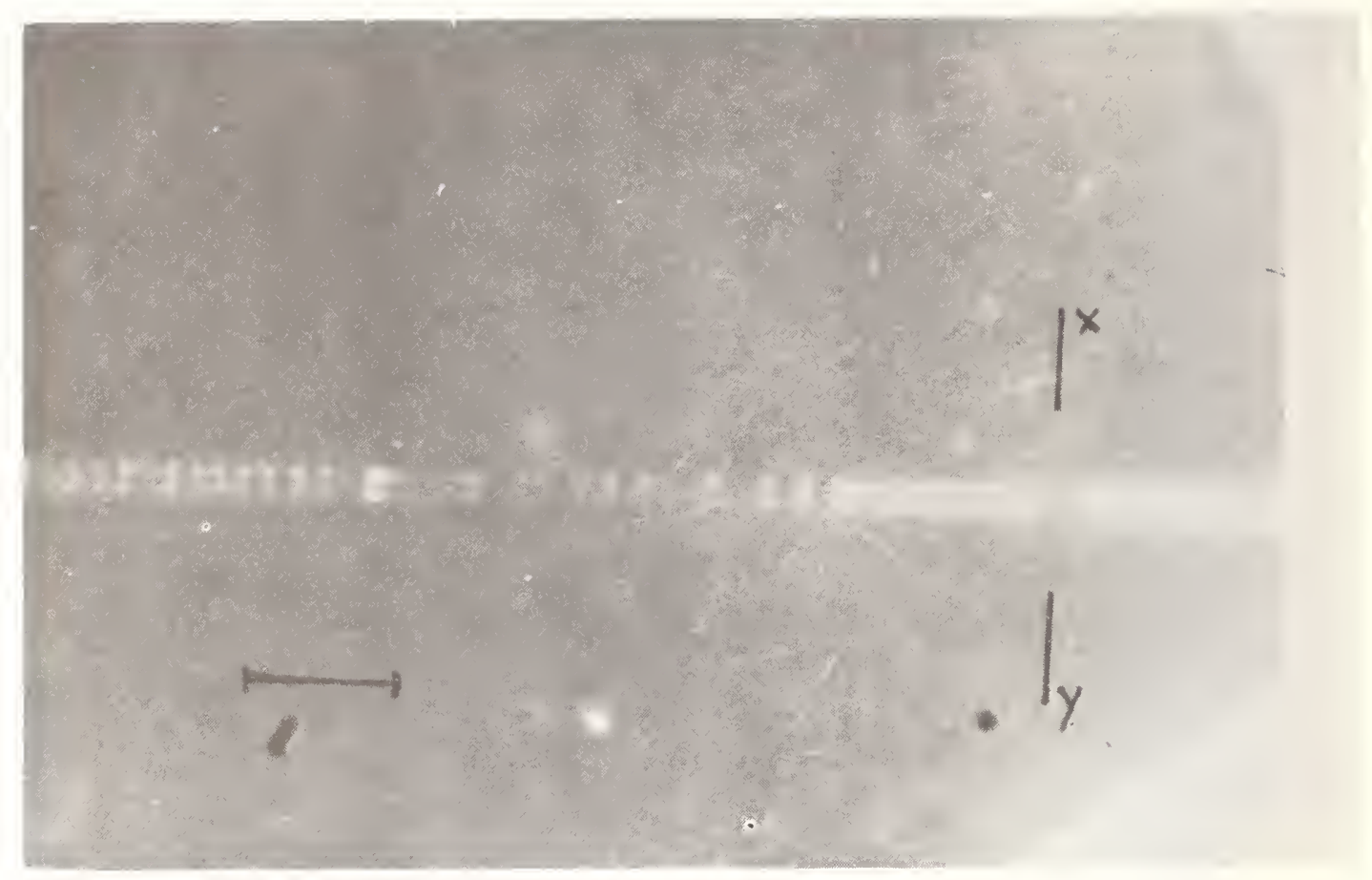

Fig. 4 Image of the filament in a polarization interferometer.

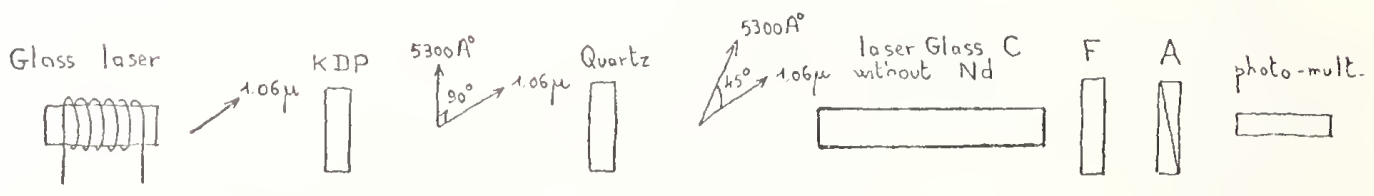

Fig. 5 Experimental arrangement used to determine $\mathrm{n}_{2}$.

COMUENTS ON! PAPER TY JEAN DAVIT

In the meceding paner, average energ" denset" values were awoted. The localized energ" densit" at the point of filanent formaten mall be much higher than the quoted value. Two tupes of experimental conkiqurations mere omploned, one in which the beam was focused in an external scomple, anc' one in whech a paralle bean was complifed un a pumped annlefier rod which was itsefs the experimental sanple. The results were edentecal in the twa cases. The detailed filamentari danage structure ohserved was indenenient of the sample eenth and the derection of irradiation. Although occasionally a visible plasma "as produced at the entrance surface of the scompe, no nore thair a few percent of the incedent radiation was absorbed in the peasma, due to the short duration $(2$ nsec. $)$ of the pulse.

On high aualitu glasses I glasses $A$ and $C$, chamage threshold measurements are reproducible to $\pm 5^{\circ}$, while on alass of poor ontecal qualit,. i.c. containina striac, a considerable variation in damagethreshold was seen. This results hom the distortion of the wave firont, which prevents the formation of an intense welk defined focal spot. For a given composition, the best auality glass show the lowest damage tirestiold.

To scomarze, the detailed description of the filanentaru damage abserved in the samples, a tube-like structure was homed in which the rehractive index was greater than that of the surrounding glass bu 0.03 (en units of refractive index). weth a deameter 0.9 wm. This tube extended from one mon to 30 an in length. Withen the tuhe, intermitent trains of bubble-lete iractures, senarated bu one wm spacing, were observed. These trains threaley vere of one mon lenath. The oriain of the region of increased refractive index mal be due to a permanent change of whase, as monosed bu Shen, or a photodielectric ciffect, as recentely proposed hy workers at the Sele Telenione Laboratory in Hoinde?. 


\title{
Measurement of the Nonlinear Index $n_{2}$ of Glass using Picosecond Pulses
}

\author{
Michel A. Duguay and John W. Hansen \\ Bell Telephone Laboratories, Incorporated \\ Murray Hill, New Jersey 07974 \\ U.S.A.
}

Using picosecond pulses from a mode-locked Nd:glass laser and the ultrafast Kerr cell apparatus we measure the nonlinear index $\mathrm{n} 2 \mathrm{~B}$ of $\mathrm{BK} 7$ and LaSF7 glass. On the assumption that the AC Kerr effect in glass is predominantly of electronic origin we derive the value $\mathrm{n}_{2} \approx 3 \times 10^{-22} \mathrm{~m}^{2} / \mathrm{v}^{2}\left(2.7 \times 10^{-13}\right.$ esu) for the nonlinear index $\mathrm{n}_{2}$ of $\mathrm{BKT}$ glass. Laser glass is assumed to have about the same $\mathrm{n}_{2}$. At power densities $\sim \mathrm{I} \mathrm{GW} / \mathrm{cm}^{2}$ this gives rise to refractive index increases of I $^{-6}$; this is sufficient to explain the appearance of self-focussing in glass laser rods which leads to the formation of long fossil tracks of microscopic bubbles. In addition, $n_{2}$ leads to pulse spectral broadening and gain limiting.

Key Words: AC Kerr effect in glass, frequency broadening, light induced birefringence, nonlinear index $n_{2}$, picosecond pulses, self-focussing, stop-motion photography of light pulses.

\section{Introduction}

When a powerful laser pulse propagates through an isotropic dielectric it induces changes in the refractive index described to first order by the equations.

$$
\begin{aligned}
& n_{\|}=n_{0}+n_{2} E^{2} \\
& n_{\perp}=n_{0}+n_{2 \perp} E^{2} \\
& n_{\|}-n_{\perp}=n_{2 B} E^{2}
\end{aligned}
$$

Here, $n_{0}$ is the usual low signal refractive index, $E$ is the root-mean-square value of the plane polarized optical field $\vec{E}, n \|\left(n_{1}\right)$ is the refractive index seen by light polarized parallel (normal) to $\overrightarrow{\vec{F}}$, and $n_{2 \mathrm{~B}} \overrightarrow{\mathrm{l}} \mathrm{s}$ the nonlinear birefringence index. In certain materials (such as $\mathrm{CS}_{2}$ for example) the constant $\mathrm{n}_{2 \mathrm{~B}}$ is approximately equal to $B \lambda$, where $B$ is the DC Kerr constant of the material, and $\lambda$ the measuring wavelength in vacuo (this relation does not hold in general).

The importance of the nonlinear index $n_{2}$ in lasers stems from the fact that the changes in refractive index $5 n\|=n\|-n_{0}$ can lead to self-focussing $[1,15]^{1}$ and frequency broadening [2] in laser rods. The coefficient $n_{2 B}$ is also of special interest because the light induced birefringence can be used in the ultrafast Kerr cell [3] to allow the photographing of light pulses propagating in various media [4].

1 Figures in brackets indicate the literature references at the end of this paper. 
In our experiment we measured the nonlinear birefringence index $\mathrm{n}_{2 \mathrm{~B}}$, and by making an assumption about the mechanism involved, we deduce the value of $n_{2}$. ${ }^{2}$ The experimental set-up has been described in Ref.[3]. Infrared $(\lambda=1.06 \mu)$ pulses from a modelocked Na:glass laser are used to induce birefringence in glass samples $2-5 \mathrm{~cm}$ in length. The $1.06 \mu$ pulses are $8 \mathrm{psec}$ in duration. [2] Green light pulses derived from them by second harmonic generation in KDP have a duration of 26 psec. The green pulses are used to probe the induced birefringence. The glass sample is placed between two crossed polarizers, and the whole arrangement acts like an ultrafast Kerr cell. [3] The green pulses are used to measure the transmission of the ultrafast Kerr cell as a function of time.

We have measured $\mathrm{n}_{2 \mathrm{~B}}$ for the Schott glasses type BK7 and LaSF7. The curve for BKT is shown in Fig. 1. The transmission is plotted in arbitrary units. Typical transmission are $\sim 0.03 \%$ for peak power densities $\sim 0.5 \mathrm{GW} / \mathrm{cm}^{2}$ in the $1.06 \mu$ picosecond pulses. When we replace the glass sample by a $1 \mathrm{~cm} \mathrm{CS}_{2}$ cell typical transmissions are $10 \%$. Since the $\mathrm{n}_{2 \mathrm{~B}}$ of $\mathrm{CS}_{2}$ is known to be: [5]

$$
\mathrm{n}_{2 \mathrm{~B}}\left(\mathrm{CS}_{2}\right)=2.3 \times 10^{-20} \mathrm{~m}^{2} / \mathrm{v}^{2}=2 \times 10^{-11} \mathrm{esu},
$$
we can use the $\mathrm{CS}_{2}$ curves as a calibration in order to obtain absolute values for $\mathrm{n}_{2 \mathrm{~B}}$
of the glasses.

We have found this way:

$$
\begin{aligned}
\mathrm{n}_{2 \mathrm{~B}}(\mathrm{BK} 7 \text { glass }) & =(2 \pm 1) \times 10^{-22} \mathrm{~m}^{2} / \mathrm{V}^{2} \\
\mathrm{n}_{2 \mathrm{~B}}(\text { LaSF7 glass }) & =(6 \pm 2) \times 10^{-22} \mathrm{~m}^{2} / \mathrm{v}^{2}
\end{aligned}
$$

(Following Ref. [10] we use SI units. The conversion factor is $1 \mathrm{~m}^{2} / \mathrm{V}^{2}=0.9 \times 10^{9}$ esu, so that $n_{2 B}(B K 7)=(2 \pm 1) \times 10^{-13}$ esu and $n_{2 B}(\operatorname{LaSF} 7)=6 \times 10-13$ esu $)$.

Also, from the fact that the curves for these two glasses have the same shape as the curve for $\mathrm{CS}_{2}$, we are able to establish an upper limit on the relaxation time $\tau$ associated with $\mathrm{n}_{2 \mathrm{~B}}$ in glasses viz:

$$
\tau(\text { glass })<5 \text { psec. }
$$

The very fast relaxation time of the induced birefringence and other evidence from frequency broadening in self-focussing [6] support the thesis advanced by several workers $[7,2,6]$ that the mechanism at work here is the electron cloud distortion mechanism. Another mechanism that could possibly be at work in glass in the collective mechanism proposed by Hellwarth. [8] There is, however, very little experimental evidence on the collective mechanism in solids, and for simplicity, we shall assume, henceforth that the effect is purely electronic in glass. If we assume a purely electronic mechanism for $\mathrm{n}_{2 B}$, we can use the work of Buckingham et al. [9, 10] to estimate the $n_{2 B}$ of a material from a knowledge of its refractive index. Buckingham et al. [9,10] have measured the birefringence induced in each molecule of a gas by a DC electric field. Each molecule possesses a hyperpolarizability coefficient $y$ which is a measure of the birefringence induced by the field. The following formula connects the bulk property $n_{2 B}$ (assumed to be of purely electronic origin) with molecular $\gamma$ :

$$
n_{2 B}(\text { electronic })=\frac{\left(n_{0}^{2}+2\right)^{4}}{8 \ln _{0}} \frac{1}{6 \varepsilon_{0}} \frac{N}{V} \gamma
$$

where $n_{0}$ is the refractive index, $\varepsilon_{0}=8.85 \times 10^{-12} \mathrm{Fd} / \mathrm{m}$ is the capacitivity of vacuum, and $\mathrm{N} / \mathrm{V}$ is the number of molecules per $\mathrm{m} 3$. For $\mathrm{SiO}_{2}$, the main constituent of $\mathrm{BK}$, a 
$\gamma \sim 10^{-61} \mathrm{c}^{4} \mathrm{~m}^{4} \mathrm{~J}^{-3}$ is not unreasonable. [2] With $\mathrm{n}_{\mathrm{O}}=1.5$ at $1.0 \dot{6} \mu$ we find:

$$
\mathrm{n}_{2 \mathrm{~B}}(\mathrm{BK} 7 \text { glass, calculated }) \sim 1.0 \times 10^{-22} \mathrm{~m}^{2} / \mathrm{v}^{2}
$$

which compares favorably with Eq. (5). For LaSF7 we have $\mathrm{n}_{\mathrm{O}}=1.89$ and using the same $\gamma$ we find:

$$
\mathrm{n}_{2 \mathrm{~B}} \text { (LaSF7 glass, calculated) } \sim 2.5 \times 10^{-22} \mathrm{~m}^{2} / \mathrm{v}^{2}
$$

which again compares favorably with Eq.(6). A correlation between $\mathrm{n}_{2 \mathrm{~B}}$ and $n_{0}$ can be clearly seen here. Since laser glass has $n_{0}=1.50$, for discussions pertaining to self-focussing and frequency broadening in laser glass, it is probably reasonable to assume:

$$
\mathrm{n}_{2 \mathrm{~B}}(\text { laser glass }) \approx \mathrm{n}_{2 \mathrm{~B}}(\mathrm{BK} 7 \text { glass })
$$

We shall assume so in the following. (Measurements of $\mathrm{n}_{2 \mathrm{~B}}$ of laser glass itself are in progress and shall be reported in a future publication.)

\section{Self-Focussing in Glass}

In the electron cloud distortion model the relation between $\delta \mathrm{n}_{\|}=\mathrm{n}_{\|}$- $\mathrm{n}$ and $\delta \mathrm{n}_{\perp}=\mathrm{n}_{\perp}-\mathrm{n}$ is $[9]$

$$
\frac{\delta n_{\|}}{\delta n_{\perp}}=+3
$$

From this and Eqs.(1)-(3) we deduce:

$$
\mathrm{n}_{2}=1.5 \mathrm{n}_{2 \mathrm{~B}}
$$

Assuming that $\mathrm{n}_{2}$ is predominantly of electronic origin in glass, we therefore have:

$$
\begin{gathered}
\mathrm{n}_{2}(\text { BKT glass }) \approx 3 \times 10^{-22} \mathrm{~m}^{2} / \mathrm{v}^{2} \\
\mathrm{n}_{2}(\text { LaSF7 glass }) \approx 9 \times 10^{-22} \mathrm{~m}^{2} / \mathrm{v}^{2}
\end{gathered}
$$

Assuming that laser glass has the value $\mathrm{n}_{2}=3 \times 10^{-22} \mathrm{~m}^{2} / \mathrm{v}^{2}$ we can now calculate the self-focussing length in laser rods. In the neglect of diffraction, the selffocussing length given by Kelley [11] is:

$$
z \text { (focus) }=\frac{1}{4} b \sqrt{\frac{n}{n_{2}}} \frac{1}{E}
$$

where $\mathrm{b}$ is the beam parameter (the beam diameter for a beam of Gaussian profile, i.e., TEM mode). In mode-locked Nd:glass oscillators and $30 \mathrm{nsec}$ pulse oscillatoramplifier systems, power densities $P$ of $\sim \mathrm{GW} / \mathrm{cm}^{2}$ are typical. [12] The spatial power density distribution frequently contains hot spots of $\sim \mathrm{mm}$ in diameter. Assuming $\mathrm{n}_{2}=3 \times 10^{-22}$, we then have $8 n_{\|}=1.1 \times 10^{-6}$ and: 


$$
\begin{gathered}
\mathrm{Z} \text { (focus) }=29 \mathrm{~cm} \\
\text { for } \mathrm{b}=1 \mathrm{~mm} \text { and } \mathrm{P}=1 \mathrm{GW} / \mathrm{cm}^{2}
\end{gathered}
$$

In large single picosecond-pulse glass laser amplifier systems power densities of I TW/ $\mathrm{cm}^{2}$ have been reported. [13] In this case we have: $\delta \mathrm{n}_{\|}=1.1 \times 10^{-3}$ and

$$
\begin{gathered}
\mathrm{Z} \text { (focus) }=0.91 \mathrm{~cm} \\
\text { for } \mathrm{b}=1 \mathrm{~mm} \text { and } \mathrm{P}=1 \mathrm{TW} / \mathrm{cm}^{2}
\end{gathered}
$$

These self-focussing distances should be compared with the observations of selffocussing in laser rods for nanosecond [1,15] as well as picosecond pulses [13,6]. From Eq. (15) it is clear that for a given $\mathrm{n}_{2}$ and power density, the self-focussing length can only be increased if more spatially uniform beams are used (i.e., larger $\mathrm{b}^{\prime}$ s in $\mathrm{Eq} \cdot(15)$ ). With beams of present quality self-focussing can be expected at power densities over $2 \mathrm{GW} / \mathrm{cm}^{2}$.

\section{Frequency Broadening}

As a powerful pulse propagates through glass, it modulates the index and this, in turn, leads to frequency modulation or spectral boradening of the pulse. The leading edge of a pulse will be frequency down-shifted and the lagging edge up-shifted by an amount equal to:

$$
\Delta v= \pm \frac{L}{\lambda} \frac{\delta n}{\delta t}
$$

where $\lambda$ is the wavelength in vacuum, $\delta \mathrm{n} / \delta \mathrm{t}$ is the rate of change of $\mathrm{n}$ with time $\left(=\mathrm{d}\left(\mathrm{n}_{2} \mathrm{E}^{2}\right) / \mathrm{d} t\right)$, and $\mathrm{L}$ is the distance over which the pulse propagates without suffering severe distortion. $[14]$. As a numerical example, we give: $P=10 \mathrm{GW} / \mathrm{cm}^{2}, \delta \mathrm{n}=11 \times 10-6$, $I=10 \mathrm{~cm}, \lambda=10^{-4} \mathrm{~cm}$, $\delta t=1 \mathrm{psec}$. Then:

$$
\Delta v= \pm 1.1 \mathrm{THz}= \pm 37 \mathrm{~cm}^{-1}
$$

which is a considerable frequency shift. Such shifts are believed to account for the $100 \mathrm{~cm}^{-1}$ spectral width of the mode-locked Na:glass laser, and the very broad spectra observed in self-focussed regions in glass. This dynamic spectral boradening is also believed to lead to gain limiting [2] in high peak power amplifiers.

\section{Ultrahigh Speed Photography of Picosecond Light Pulses}

As an application of the light induced birefringence, we show in Fig. 2 photograph of a green light pulse in flight through a cell of water containing a slight amount of milk in suspension. [4] The photograph is taken from the side by a camera placed behind an ultrafast shutter of 10 psec framing time. [3] In this case, $\mathrm{CS}_{2}$ was used in the shutter, but in the future glass could be used for response times faster than 2 psec (the response time [7] of the electronic effect may be as fast as 10-15 sec). The ultrafast camera could be used in studies of damage in glass. With proper time and space resolution, laser pulses could be photographed as they are self-focussing and freqeuncy broadening. It may also be possible to photograph the time development of regions of bubble and inclusion damage. 
[1] Zverev, G. M., Levchuk, E. A., Maldutis, E. K., and Pashkov, V. A., Self-focussing of laser radiation in active media and in non-linear crystals, Optica i spektroskopia, vol. 27, (November 1969), pp. 1060-1062.

[2] Duguay, M. A., Hansen, J. W. , and Shapiro, S. L., Study of the Nd: glass laser radiation by means of two-photon fluorescence, IEEE J. Quantum Electronics, Vol. QE-6, (October 1970).

[3] Duguay, M. A. and Hansen, J. W., An ultrafast light gate, Appl. Phys. Letters, Vol. 15, (Sept. 1969) pp. $192-194$.

[4] Duguay, M. A. and Hansen, J. W., Ultrahigh speed photography of picosecond light pulses, submitted to Applied Physics Letters, May 28 $(1970)$.

[5] Paillette, M., Mesure of I'effet Kerr induit par une onde lumineuse intense, Compt. Rendus Acad. Sc. Paris, Vol. 262B, (Jan. 1966) pp. $264-267$.

[6] Alfano, R. R. and Shapiro, S. L., Observation of self-phase modulations and small scale filaments in crystals and glasses, Phys. Rev. Letters, Vol. 24 (March 1970) pp. 592-594.

[7] Brewer, R. G. and Lee, C. H., Selftrapping with picosecond light pulses, Phys. Rev. Letters, Vol. 21, (July 1968) pp. $267-270$.

[8] Hellwarth, R. W., Quantum molecular theory of light scattering spectra, J. Chem. Phys.

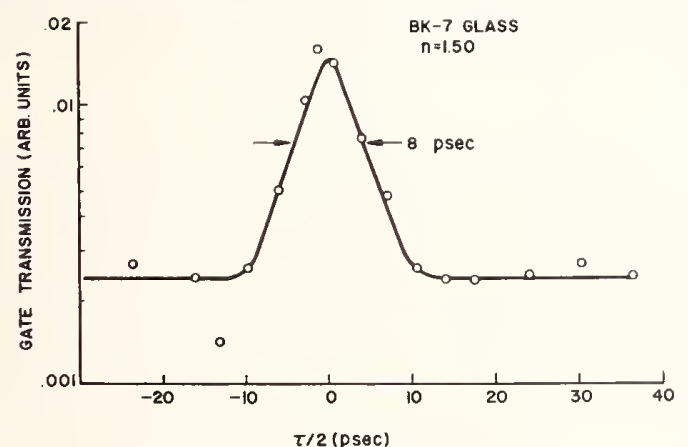

Fig. 1
[9] Buckingham, A. D. and Dunmur, D. A., Kerr effect in noble gases and sulfer hexafluoride, Trans. Faraday Soc., Vol. 64, (1968) pp. 1776-1783.

[10] Buckingham, A. D. and Orr, B. J., Kerr effect in methane and its four fluorinated derivatives, Trans. Faraday Soc., Vol. 65, (March 1969) pp. 673-681.

[11] Kelley, P. L., Self-focussing of optical beams, Phys. Rev. Letters, Vol. 15, (Dec. 1965) pp. 1005-1008.

[12] Young, C. G. and Woodcock, R. F., Laser-induced damage in glass, Damage in Laser Glass, ASTM STP 469 , American Society for Testing and Materials (1969) pp. 86-89.

[13] Gobeli, G. W., Bushnell, J. C., Peercy, P. S. and Jones, E. D., Observation of neutrons produced by laser irradiation of lithium deuteride, Phys. Rev., Vol. 188, (Dec. 1969) pp. 300-302.

[14] Joenk, R. J. and Landauer, R., Laser pulse distortion in a nonlinear dielectric, Phys. Letters, Vol. $24 \mathrm{~A}$ (Feb. 1967) pp. 228-229.

[15] Davit, J., Filamentary damage in glasses (paper in this issue).

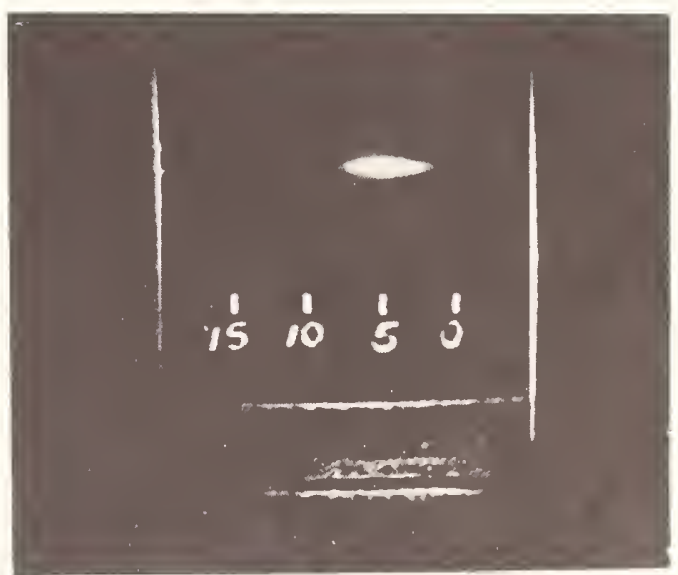

Fig. 2 Stop-motion photograph of a green light pulse propagating through a cell containing a colloidal suspension of milk particles in water. The pulse is seen through its scattered light. An ultrafast light gate served as a camera shutter with a framing time of 10 psec. Glass could eventually be used in shutters 10-100 times faster. The pulse is travelling from right to left. The scale is in millimeters. 
There was considerable discussion on the question of which self-focusing mechanisms were operative in the $30 \mathrm{usec}$ time reaime. There uas general agreement that in the picosecond time regime, the dominant self-focusing mechanism was the Kerr effect, either electronic, nuclear, or due to nolecular libration. Dr. Hellwarth pointed out that the last should be detectable from a detaifed analus is of the structure of Raman spectra. Although it is well established that there is sufficient time in 30 nsec pusses for efectrostrictive trapning to occur, the fact th? t observed filaments onfu last for times like $100 \mathrm{psec}$. raises doubts that the clectrostrictive mechanism is the only one operating. An additional complication arises from the fact that without extreme care, manif 30 nsec pulses contain sub-nanosecond intensity fluctuations. The question of the exact mechanism of self-focusing in the time reaine of 1 to $30 \mathrm{nsec}$ was eqht uriesolved.

[Etitor's note: Due to time limitations, the follouing comment was submitted in writing bu Dr. Charles Wang of the Ford Hotor Companu Scientific Research Stabj]

This comment involves (1) the conversion of the susceptibility coefficients from Mks units to cas units; 121 the magnitude of $n_{2}$. "the coeficient of intensitu-dependent index"; and (3) the experimental determination of $h_{2}$ through ontical kerr ehject measurements.

(1) The conversion from iks to cos units is discussed in lininck, Terhune and wang, Proc. IEEE 54, 1357 $(1966))$

$$
x^{(3)}\left(\text { mks) }=\frac{4^{\pi}}{9} \times 10^{-8} x^{(3)}\right. \text { (esu) }
$$

it is VOT $10^{-9}$ as given bul Dugua!!.

(2) The coefficient of the intensitu-dependent index n? is defined as (Kellu, Phus. Rev. Letters 15, $1005(1965))$

$$
\delta n=\frac{1}{2} n_{n}|E|^{2}
$$

where $|E|$ is the amplitute of the clectric field and sn is the change in the resractive index. This coekicient is related to the usual suscentibilitu constants $x(3) \mid-\omega, w,-w, w)$ as follows (Uang, Pinis. Rev. 152, 149 (1966)):

$$
\delta n=\frac{6 \Gamma}{n} x^{(3)}(-\omega, \omega,-\omega, \omega)|E|^{2}
$$

At ireauencies small comared to an! of the electronic resonance freauencjes of the system, the electronic contribution to $x^{(3)}(-\omega, \omega,-\omega, \omega)$ is the sane as that to $x(3)(-3 \omega, \omega$, $\omega$, $\omega)$ in the experiments of third harmonic generation. Thus, the electronic part of $\delta_{n}$ can be accuratell calculated using the available data of $x(3)(-3 w, w, w, w)$. Tire value of $x$ (3) for borosilicate glass is (uana, Phus. Rev. B. Sept. 1970): $x^{(3)}=1.3 \times 10^{-11}$ esu. This gives

$$
\delta_{n}=1.63 \times 10^{-13}|E|^{2}
$$

(3) It is well bnown that reliable measurements of optical kerr efiect in solids is extremelu difificult, as an bire fringence (due to local stress, etc.) vill obscure the measurements. The disherence in $n_{2}$ measured by Davit for glass $A$ and glass $C$ must thus be interpreted witi caution. 


\title{
Absorbing Centers in Laser Glasses
}

\author{
Herbert S. Bennett
}

National Bureau of Standards

Washington, D.C. 20234

\begin{abstract}
Some metallic and dielectric inclusions in glass laser rods may absorb an appreciable amount of the laser radiation and thereby may produce major stresses within the glass host. The radial and tangential stress components, the stress birefringence, the optical path length change for a probing light ray passing near the inclusion, and the "lens" effect are computed. The dependence of the maximum value of the tensile stress upon the size of the inclusion and upon the physical properties of the host is examined.
\end{abstract}

Key Words: Antimony, heat conduction, laser damage, laser glasses, platinum, stress components, thermoelastic theory。

\section{Introduction}

One of the severe problems encountered in high-power-solid-state laser systems is the thermal damage to laser rods and optical elements. One such type of damage is throught to arise from metallic or dielectric inclusions. Such inclusions may absorb an appreciable amount of incident laser radiation and thereby may undergo thermal expansion. This produces major stresses within the host material. Estimating such thermal properties requires the consideration of solutions to the heat diffusion equation and the thermal stress equations with appropriate boundary conditions. In particular, the optical path length change for a probing light ray passing near the absorbing center, the radial and tangential stress components, and the changes of the refractive index for radially polarized and tangentially polarized light due to the thermal stress field are computed. The dependence of the maximum value of the tensile stress upon the size of the inclusion and upon the physical properties of the host is examined. The feasibility of using optical techniques to detect metallic and dielectric inclusions in laser materials before they cause damage also is studied. These computations suggest that the use of laser pulse widths of the order of microseconds or longer may be more promising for the detection of small incipient absorbing centers than the use of nanosecond pulse widths. In addition, the lens effect is estimated.

\section{Description of the Mode1}

The results sumnarized in this paper are based upon a linear theory and upon a model whose elastic, thermal, and optical properties are constants. The values used for these properties are valid for temperatures near $300 \mathrm{~K}$ and for small strains. This treatment is not expected to be correct near the onset of damage. The problem is certainly a nonlinear one near the region of catastrophic damage. Whenever any of the assumptions become invalid, then the results should be viewed as suggesting trends in the behavior of the system. The inclusions which occur in laser glasses are most likely not the spheres for which the model is formulated. They could be irregularly shaped globules. Some platinum inclusions occur as hexagonal platelets. Even though the model does not take into account such geometrical aspects, one hopes that it does give a reasonable description of the actual system. One also hopes that the manner in which it suggests one should proceed to increase the damage threshold, though probably not quantitatively correct, is qualitatively correct.

A model to represent the behavior of absorbing centers in laser materials is developed. Before the thermal properties of the absorbing center-host system can be discussed quantitatively, it is first necessary to solve the time dependent heat diffusion equation for the temperature and the themal stress equations with appropriate boundary conditions for the stress components. This is discussed in detail in Ref. 1 and Ref. 2. 
The model contains many physical assumptions which are necessary to render the problem solvable. The major assumptions are summarized here.

a) The inclusion is a sphere of radius $r$ and is always in good thermal contact with the host. The effects of shape and orientation to the incident radiation are neglected.

b) The host material is isotropic, continuous and of infinite extent. It also is initially at an ambient temperature $\mathrm{T}$ and free from all stresses and strains. Because the energy content of the incident radiation is ${ }^{\circ}$ finite, the latter statement requires the temperature to be $T_{0}$ at infinity and all stresses and strains to vanish at infinity. The distribution and nature of microcracks and optical imperfections are not treated in the model.

c) The linear-thermal-elastic equations are assumed to give a reasonable description of the processes which ultimately may lead to catastrophic damage. These equations are coupled equations relating the temperature and the displacement vector from which the stresses and strains are computed. They are valid only when a local temperature exists and when distances are larger than atomic dimensions $\left(\sim 10^{-8} \mathrm{~cm}\right)$. A relaxation time $t$ for the definition of a local temperature $\mathrm{T}$ is approximately the reciprocal of a characteristic vibration frequency of the material. These relaxations times $t$ for $\mathrm{Pt}, \mathrm{Sb}, \mathrm{Al}_{2} \mathrm{O}_{3}$, and the laser glasses are about $10^{-13} \mathrm{~s}$ to $10^{-12} \mathrm{~s}$. Hence, the equations are physically meaningful only when times $t$ are much larger than $10^{-12} \mathrm{~s}$.

d) It is assumed that the radiation of heat by the center-host interface and by the heated glass close to the absorbing center may be neglected in the thermal-elastic equations. The 1aser beams studied in Ref. 1 and Ref. 2 contain energy fluxes at least $10^{4}$ times greater than the energy flux produced by a black body at $600^{\circ} \mathrm{C}$. The calculations of Ref. 1 also show that the energy flux due to thermal conduction greatly exceeds the energy flux due to radiation for times less than one second. Because the temperature is close to the ambient temperature whenever the pulse width of the laser beam is less than a milisecond and whenever the time after cessation of the laser beam is greater than a second, the long time behavior is not in the region of practical interest for detecting damage centers before they cause damage. Hence, it is assumed that all times are less than a second.

e) The linear-thermal-elastic equations contain a coupling term and an inertia term. These two terms may be neglected whenever the three characteristic times which occur in the absorbing center-host system satisfy a set of inequalities. These times are the following. The pulse width $\tau$ of the incident radiation determines in part the rapidity of heat generation. The characteristic relaxation time for temperature equilibration (thermal diffusion) is $t_{\mathrm{T}} \sim\left(\mathrm{r}^{2} / \mathrm{a}^{2}\right)$, where $\mathrm{r}$ is the radial distance from the center of the inclusion and $\mathrm{a}^{2}$ is the thermal diffusivity. The characteristic mechanical time required for the production of stress waves is $t_{M} \sim(r / v)$, where $v$ is the speed of propagation of elastic waves. Boley and Weiner have demonstrated that when $\tau>>t_{M}$ and $t_{T}>>t_{M}$, then the coupling and inertia terms are small compared to the other terms in the equations. [3] ${ }^{\mathrm{T}}$ The above inequalities are reduced to inequalities containing the pulse width $\tau$ and the radial distance r; [1] namely: $\tau>>2.8 \times 10^{-6} \mathrm{r}(\mathrm{s} / \mathrm{cm})$ and $\mathrm{r}>>6.7 \times 10^{-7} \mathrm{~cm}$. It is assumed that these inequalities are satisfied and thereby that the coupling and inertia terms may be neglected.

In sumary, the following inequalities describe the regions for which the mode1 is physically meaningful:

$$
\begin{aligned}
& 10^{-3} \mathrm{~s}>\tau>10^{-12} \mathrm{~s}, 1 \mathrm{~s}>\mathrm{t}>10^{-12} \mathrm{~s} \\
& \mathrm{r}>6.7 \times 10^{-7} \mathrm{~cm}, \text { and } \tau>2.8 \times 10^{-6} \mathrm{r}(\mathrm{s} / \mathrm{cm}) .
\end{aligned}
$$

\section{Results and Conclusions}

The numerical results which the model developed in Ref. 1 predicts, are summarized here. Among the many input parameters, the absorptance $A(\lambda, T)$ of the center-host interface is perhaps most sensitive to the initial thermal contact and surface conditions of the inclusion and host. The wavelength is $\lambda$. The numerical results are given for the case in which $A(\lambda, T)=1$. This presents no additional problem because the temperature $T_{h}(r, t)$, the optical path length change $\Delta L$ for a probing light ray passing near the inclusion, the stress components $\sigma_{\text {r }}$ and $\sigma_{\forall \theta}=\sigma_{\text {rop }}$ for the spherical coordinates $r, \forall$, and $\varphi$, and the changes in the refractive index due to the thermal stress field for radially polarized light $\Delta n_{r}$ and for tangentially polarized light $\Delta n_{\theta}$ are all directly proportional to the absorptance.

The model is used to study three questions. First, how the maximum tangential-tensile stress varies as a function of the radius of the spherical inclusion for a fixed energy density $E_{\mathrm{I}}$ and pulse width of the laser beam $\tau$. Second, how the maximum of the tangential-tensile stress varies as a function of the thermal conductivity and the thermal expansion coefficient of the host. And third, if the maximum temperature of the inclusion is limited to a fixed value, $\mathrm{T}_{\mathrm{h}}(\mathrm{r}, \tau)=600^{\circ} \mathrm{C}$, how the parameters of the incident laser beam should be varied to increase the probability of detecting by optical techniques a small incipient absorbing center before it causes damage. Optical techniques to detect small inclusions become more promising, the greater $\Delta \mathrm{I}, \Delta \mathrm{n}_{\mathrm{r}}$, and $\Delta \mathrm{n}_{\theta}$ are. 
The bulk thermal, elastic, and optical properties for the laser material used in these calculations are representative of two $\mathrm{Nd}$-doped laser glasses manufactured domestically. The inclusions are $\mathrm{Pt}, \mathrm{Sb}$, and polycrystalline $\mathrm{Al}_{2} \mathrm{O}_{3}$ absorbing spheres.

The variation of the maximum tensile stress $\sigma_{\text {or }}$ (max-tensile) as a function of $r$ for a laser beam having an energy density $E_{T}=20\left(\mathrm{~J} / \mathrm{cm}^{2}\right)$ and $a^{\varphi p p u l s e ~ w i d t h ~} \tau=30$ ns is studied. ${ }^{\circ}$ When $10^{-4} \mathrm{~cm}>\mathrm{r}>5 \times 10^{-5} \mathrm{~cm}$, the maximum tensile stress exceeds by as much as a factor of two the theoretical strength of the glass $\left(6 \times 10^{9} \mathrm{~N} / \mathrm{m}^{2}\right)$. The calculations show that sb and highly absorbing $\mathrm{Al}_{2} \mathrm{O}_{3}$ inclusions are more likely to cause damage than Pt. The maximum tensile stresses for Sb and $\mathrm{Al}_{2} \mathrm{O}_{3}$ inclusions are about $20 \%$ larger than those for Pt. It is interesting to note that one domestic manufacturer no longer uses $\mathrm{Sb}_{2} \mathrm{O}_{3}$ as a fining agent. [4]

These results demonstrate that submicron-sized inclusions have the greatest probability to cause damage in laser glass hosts. Experimental measurements performed by industrial researchers [5] have verified tentatively this theoretical result. Very large $\left(r_{0}>10^{-4} \mathrm{~cm}\right)$ and very smal1 ( $\left.r_{0}<5 \mathrm{x} 10^{-5} \mathrm{~cm}\right)$ inclusions are not likely to produce damage.

The maximum tensile stress as a function of the thermal conductivity $K_{\text {and }}$ the thermal expansion coefficient $\alpha_{\mathrm{b}}$ is also studied for $r=5 \times 10^{-5} \mathrm{~cm}, \tau=30 \mathrm{~ns}$, and $\mathrm{E}_{\mathrm{T}}=20\left(\mathrm{~J} / \mathrm{cm}^{2}\right)$. It is found that increasing $\mathrm{K}$ 辛rom $0.008\left(\mathrm{~W} / \mathrm{cm}^{\circ} \mathrm{C}\right)$ to $0.04\left(\mathrm{~W} / \mathrm{cm}^{\circ} \mathrm{C}\right)$ with al1 remaining properties kept constant decreases the maximum tensile stress from about $9 \times 10^{9}\left(\mathrm{~N} / \mathrm{m}^{2}\right)$ to $4 \times 10^{9}\left(\mathrm{~N} / \mathrm{m}^{2}\right)$. Again, all the other properties of the host are kept the same except for the thermal expansion coefficient. The maximum tensile stress is studied then as a function of $\alpha_{h}$. The maximum tensile stresses are found to have minimum values for $10 \times 10^{-6}\left(1 /{ }^{\circ} \mathrm{C}\right)>\alpha_{h}>8 \times 10^{-6}\left(1 /{ }^{\circ} \mathrm{C}\right)$. Values of $\alpha$ outside this range yield larger stresses. They also are slowly varying functions of the thermal expansion coefficient. Increasing $\alpha_{\mathrm{h}}$ by a factor of three produces only a 10\% variation in the maximum tensile stress. Hence, the thermal conductivity influences greatly the behavior near the region of maximum tensile stress and the thermal expansion coefficient plays only a minor role in the value of the maximum tensile stress. Among the many possible glass laser hosts, the expansion coefficients vary by about an order of magnitude from $10^{-6}\left(1 /{ }^{\circ} \mathrm{C}\right)$ to about $10 \times 10^{-6}\left(1 /{ }^{\circ} \mathrm{C}\right)$ and all the rernaining bulk elastic and thermal properties vary by only small amounts. The latter variations usually do not exceed a factor of two. Hence, extensive research on altering substantially the composition of present laser glasses to increase the damage threshold due to inclusions is not warranted by the predictions of this model. It suggests that probably at the best, a factor of two increase in the damage threshold due to inclusions could be achieved.

The model also shows that the maximum value for the tensile stress does not depend upon the Young 's modulus E in a straight forward manner as some researchers have suggested. The Lame' constants are the independent elasticity variables and changes should be discussed in terms of them. If it were possible to alter only the Young's modulus, then the stresses would increase in a monotonic fashion with increasing values of $\mathrm{E}$. But such changes are not possible in practice. The model predicts that glasses with higher $\mathrm{E}$ values are not necessarily more resistant to damage.

The optimum parameters of the incident laser beam to be used for detecting incipient damage centers before they cause damage are examined for the case in which the maximum temperature is $600^{\circ} \mathrm{C}$ at the cessation of the laser pulse. The computations indicate that pulse widths of the order of microseconds or longer heat the center more slowly and thereby produce larger optical path length changes for a probing light ray passing near the inclusion. For example, a Pt sphere with radius $\mathrm{r}-10^{-4} \mathrm{~cm}$ produces a path length change of about $2 \times 10^{-8} \mathrm{~cm}$ for $\tau=0.1 \mu \mathrm{s}$ and of about $10^{-6} \mathrm{~cm}$ for $\tau^{\circ}=100 \mu \mathrm{s}$. Researchers [4] using microsecond pulses and holography have detected $5 \mathrm{x} 10^{-3} \mathrm{~cm}$ inclusions without its causing damage. It is not known yet whether this method can be improved sufficiently to detect $10^{-4} \mathrm{~cm}$ inclusions.

Finally, the lens effect due to heated regions of the host is estimated. Whenever the refractive index increases with temperature, $\left(\mathrm{dn}_{h} / \mathrm{dT}_{h}\right)>0$, and even though the inclusion does not produce damage at its site, the heated region of the host surrounding the inclusion might focus the same laser pulse or a succeeding laser pulse which occurs after sufficiently short times and before the heated region cools. The lens effect is estimated by considering the focal length f of a spherical lens having a radius $r$ and mean refractive index $n$. The refractive index of the host is $n_{h}$ and because $\left(\mathrm{dn}_{\mathrm{h}} / \mathrm{dT}_{\mathrm{h}}\right)>0 ; \mathrm{n}_{1}>\mathrm{n}_{\mathrm{b}}$ 。 Some numerical examples for platinum inclusions are cited. When $\mathrm{r}_{0} \stackrel{\mathrm{h}}{=} 10^{-4} \mathrm{~cm}, \mathrm{E}_{\mathrm{L}}={ }^{1} 20\left(\mathrm{~g} / \mathrm{cm}^{2}\right)$, and $\tau=30 \mathrm{~ns}$, then an effective focal length due to the 1 ens effect occurs at $t \sim 3 \mu$ s and is about $f \sim 14 \mathrm{~cm}$. But the tensile stress at $t=\tau=30$ ns exceeds the theoretical strength of the host before the lens effect becomes most important. Consider now another example for Pt in which the maximum tensile stress is less than the theoretical breaking strength of the glass host. When $r_{0}=10^{-4} \mathrm{~cm}, \mathrm{E}_{\mathrm{L}}=0.33\left(\mathrm{~J} / \mathrm{cm}^{2}\right), \tau=30 \mathrm{~ns}$, and $\mathrm{T}_{\mathrm{h}}\left(\mathrm{r}_{\mathrm{O}}, \tau\right)=600^{\circ} \mathrm{C}, t$ the effective focal length attains a value of about $10^{4} \mathrm{~cm}$ for times $t \sim 3 \mathrm{~s}$. This latter focal length is very long.

Hence, the model predicts that the lens effect arising from heated inclusions probably does not cause damage. Those cases for which the maximum tensile stress is less than the theoretical tensile strength of the glass have minimum effective focal lengths which are much greater than the dimensions 
of $\mathrm{Nd}$-doped glass elements used in present laser systems. In those cases for which the minimum effective focal length is comparable to the size of the host, the tensile stress exceeds the theoretical strength of the glass and probably causes damage before the lens effect becomes large enough to heat another inclusion or to initiate an intrinsic damage mechanism such as self-focusing.

In conclusion, the model enables one to answer qualitatively many questions concerning laser damage due to absorbing centers and concerning the detection of such centers before they cause damage.

\section{References}

[1] Bennett, H. S., Inclusions in Laser Materials, to be published.

[2] Bennet, H. S., Heat Diffusion near Absorbing Centers in Laser Materials, to be published.

[3] Boley, B. and Weiner, J., Theory of Thermal Stresses, (John. Wiley and Sons, Inc., New York, 1960).
[4] Snitzer, Elias, private communication.

[5] Lee, Haynes, private communication.

COMHEVTS ON H. BENNETT'S PAPER FOLLOW THOSE OF R. HOPPER'S PAPER 
The Inclusion Problem

in Laser Glass

R. W. Hopper, C. Lee and D. R. Uhlmann

Department of Metallurgy and Materials Science

Center for Materials Science and Engineering

Massachusetts Institute of Technology

Cambridge, Massachusetts

The problem of inclusion damage in laser glass is considered anew, with attention being directed to an improved treatment of the stress around a hot inclusion particle. The existence of a layer of molten glass surrounding such particles is suggested; and the new analysis indicates a maximum tensile stress in the nearby cooler glass, rather than at the solid-liquid glasss boundary. The magnitude of this tensile stress can exceed the expected strength of the glass for a range of particle sizes under high power laser operation, and failure is then expected to occur.

The breakdown condition is shown to depend upon the flux level and pulse time of the laser beam, as well as the size and shape of the inclusion particles and various material properties of the inclusion and glass. For flux levels in the range of $1 \mathrm{j} \mathrm{cm}^{-1} \mathrm{~ns}^{-1}$ and pulse times in the range of tens of $\mathrm{ns}$, metal particles as small as $1000 \AA$ are 1 ikely to result in failure. Under most conditions of laser operation, non-metallic inclusions - even those containing large concentrations of highly absorbing ions - are expected to be safe; and under all conditions they should be appreciably less dangerous than similar metal particles.

Key Words: Glass damage, glass lasers, heat conduction, inclusion damage, lasers.

\section{Int roduction}

The problem of inclusion damage - i.e., breakdown of the glass at various discrete points- has frequently limited the use of glass lasers in high power applications. The origin of this damage has been associated with the presence in the glass of discrete second-phase inclusion particles (1-3). In a recent paper (4), the present authors considered in some detail the origin of inclusion damage, and concluded that such damage could be associated with the temperature rise of particles, or surface regions of particles, relative to the surrounding glass. The particles of greatest concern were identified as metallic, although at very high levels ceramic inclusions containing large concentrations of highly-absorbing ions could likewise result in failure.

Solutions to the heat flow problems of a perfectly conducting sphere in a medium of fintte conductivity and of the infinite composite solid were used to represent the physical situation. These

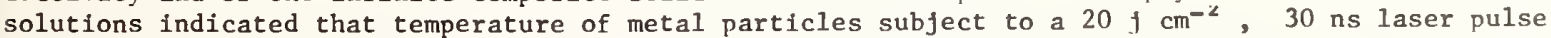
could exceed $10,000^{\circ} \mathrm{K}$ for a range of particle sizes. These high temperatures would produce stresses in the glass adjacent to the particles which can exceed the theoretical strength of the glass, and result in failure.

The effects on the breakdown condition of the flux level, pulse time, particle size and various material parameters, were considered. Specific applications were, however, restricted to laser pulses of $20 \mathrm{j} / \mathrm{cm}^{-2}, 30 \mathrm{~ns}$. In the present paper, we shall review the results of the previous study and extend them to describe phenomena occuring over a widerange of particle sizes, flux levels and pulse times. The results will be employed to delineate the ranges of particle sizes which should be viewed as dangerous for various conditions of laser operation, and to evaluate the reliability of various methods for damage testing laser glass.

In all cases, the particles will be taken as metallic, opaque at the laser frequency, and in good thermal contact with the surrounding glass. The laser pulse will be taken as a square wave 
of a given duration, incident on one side of the particles, and Lambert's Cosine Law will be assumed. Values for the assumed material parameters, which are characteristic of Pt particles in a typical Ba crown laser glass, as well as definitions of the various symbols, are summarized in the Appendix,

\section{Thermal Behavior}

The temperature of a metal particle and of the adjacent glass subject to a laser pulse has been approximated by two solutions to the heat flow problem which are appropriate for different ranges of particle size relative to the pulse time.

$$
\text { a. } R_{1} \ll\left(D_{1} t\right)^{\frac{1}{2}}
$$

For particle sizes which are small with respect to the thermal diffusion distance for the particle materials (with a given pulse time), the physical situation can well be approximated by considering perfectly-conducting spherical metal particles in a medium having the thermal conductivity of the glass. In this case, the particle temperature may be expressed (4)

$$
\begin{array}{r}
\bar{T}=\frac{3 \varepsilon_{\lambda} J R}{2 C_{v i} D_{g} m}\left[\frac{1}{q-m}\left\{1-\exp \left[\frac{D_{g} t(q-m)^{2}}{4 R^{2}}\right] \operatorname{erfc} \frac{q-m}{2 R}\left(D_{g} t\right)^{1 / 2}\right\}\right. \\
\left.-\frac{1}{q-m}\left\{1-\exp \left[\frac{D_{g} t(q+m)^{2}}{4 R^{2}}\right] \operatorname{erfc} \frac{g+m}{2 R}\left(D_{g} t\right)^{1 / 2}\right\}\right]
\end{array}
$$

where $q=3 C_{v g} / C_{v i} ; m=[q(q-4)]^{\frac{1}{2}} ;$ and it is assumed in obtaining the expression in this form that $q>4 . v g ~ v i$ Corresponding expressions for the cases when $q=4$ and $q<4$ may similarly be obtained, and in the latter case present some interesting featuries (see Ref. 5).

The temperature distribution in the glass surrounding the particle can similarly be expressed:

$$
\begin{aligned}
T_{\text {GLASS }} & =\frac{3 \varepsilon_{\lambda} J R^{2}}{2 C_{V_{i}}(R+x) D_{g} m}\left\{\frac{1}{q-m} \operatorname{erfc} \frac{x}{2\left(D_{g} t\right)^{1 / 2}}-\frac{1}{q+m} \operatorname{erfc} \frac{x}{2\left(D_{g} t\right)^{1 / 2}}\right. \\
& -\frac{1}{q-m} \exp \left[\frac{(q-m) x}{2 R}+\frac{(q-m)^{2} D_{g} t}{4 R^{2}}\right] \operatorname{erfc}\left[\frac{x}{2\left(D_{g} t\right)^{1 / 2}}+\frac{(q-m)\left(D_{g} t\right)^{1 / 2}}{2 R}\right] \\
& \left.+\frac{1}{q+m} \exp \left[\frac{(q+m) x}{2 R}+\frac{(q+m)^{2} D_{g} t}{4 R^{2}}\right] \operatorname{erfc}\left[\frac{x}{2\left(D_{q} t\right)^{1 / 2}}+\frac{(q+m)\left(D_{g} t\right)^{1 / 2}}{2 R}\right]\right\}
\end{aligned}
$$

where $\mathrm{x}$ is the radial distance from the periphery of the particle to the point in question.

$$
\text { b. } R_{1} \gg\left(D_{i} t\right)^{\frac{1}{2}}
$$

For particle sizes which are large with respect to the thermal diffusion distance, the physical situation can be approximated by treating the infinite composite solid with a flux, J, at the boundary. In this case, the thermal conductivities of the two materials are taken as those characteristic of the metal and glass. For this case, the temperature in the particle may be expressed:

$$
T_{i}=\frac{2 \varepsilon_{\lambda}\left(D_{i} D_{g} t\right)^{1 / 2} J}{k_{i} D_{g}^{1 / 2}+k_{g} D_{i}^{1 / 2}} \operatorname{ierfc} \frac{x_{i}}{2\left(D_{i} t\right)^{1 / 2}}
$$
where $x_{i}$ is the distance from the boundary into the particle material, and ierfc $y=\frac{1}{\pi} \frac{1}{2}$ exp
$\left(-y^{2}\right){ }^{-y}$ erfcy. The corresponding expression for the temperature in the glass is 


\section{$T_{g}=\frac{2 \varepsilon_{\lambda}\left(D_{i} D_{g} t\right)^{1 / 2} J}{k_{i} D_{g}^{1 / 2}+k_{g} D_{i}^{1 / 2}}$ ierfe $\frac{x_{g}}{2\left(D_{g} t\right)^{1 / 2}}$}

where $x_{g}$ is the distance from the particle into the glass.

The particle temperatures expected from eq(1) for various particle sizes and conditions of laser operation are shown in Fig. 1. With increases in flux level (and hence in energy density) for a given pulse time, the temperature increases for any given particle size, but the shape of the temperature vs. particle size relation remains unchanged. For the assumed $30 \mathrm{~ns}$ pulse duration, it is seen that temperatures in the range of $10^{4} \mathrm{o}$ are expected for a range of particle sizes and the range of particle sizes having temperatures above some given value is expected to increase with increasing flux level.

For a given energy density (Fig. 2), the expected behavior is more complex as the flux level and pulse time are separately varied. The maximum in the temperature vs. particle size relation is seen to shift to larger particle sizes with increasing pulse time (as expected for increasing thermal diffusion distances). For particle sizes much larger than the respective maxima, but still in the range where eq (1) is appropriate, the expected temeprature depends only on the energy density and hence is the same for all combinations of flux level and pulse time having a given Jt product. The magnitude of the maxima in the temperature vs. particle size relations, and indeed the magnitude of the entire relation for particle sizes in the range of or smaller than the maxima, is expected to increase with decreasing pulse time (with increasing flux level).

For a given small particle size, the temperature can be higher for a short time, high flux, pulse having a given energy density; than for a longer time, smaller flux, pulse having a larger over-all energy density. This is illustrated by the data shown in Fig 3 , where the temperature vs. particle

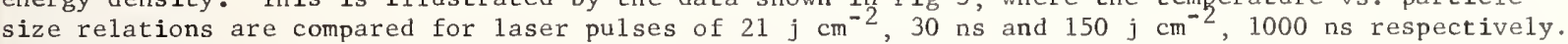

It should be recognized, of course, that the assumption of the particles being opaque at the laser wavelength is highly suspect for particle sizes smaller than a few hundred $\&$ (the $1 / \mathrm{e}$ distance for Pt, determined from measurements on thin films, is in the range of $100 \AA$ ). Further, for particle sizes much smaller than $\lambda / 10$ (about $1000 \AA$ for a Nd laser), the effective absorption cross section of the particles, which is proportional to the spectral emissivity in eqs (1) and (2), should no longer be taken as a constant. Rather, from the data presented in Born and Wolf (6), the effective $\varepsilon_{\lambda}$ should be taken as a linear function of $R_{f}$ which reaches the assumed constant value at about $R_{j}=\lambda / 10$. This decrease in the effective $\varepsilon_{\lambda}$ with decreasing particle size is associated with a decrease ${ }^{1}$ the effective absorption thickness of the particle. Since the particle temperature is proportional to $\varepsilon_{\lambda}$

[ see eq (1)], this size dependence of the effective $\varepsilon_{\lambda}$ has the effect of decreasing the particle temperature in the very small particle limit below the values estimated with a constant value of $\varepsilon_{\lambda}$, and introduces an additional size dependence into the temperature expression for this size range. $\lambda$ Consequently, the calculated temperatures shown in Figs. 1-3 for particle sizes smaller than $1000 \AA$ should be reduced by a factor of $R_{i}(\AA) / 1000$.

It should further be noted that radiation heat losses from the particles have been neglected in these calculations. This assumption may be justified by noting that Pt particles would be in thermal equilibrium with a laser beam of flux level $0.7 \mathrm{f} \mathrm{cm}^{-2} \mathrm{~ns}^{-1}$ only at temperatures about $75,000 \mathrm{~K}^{-1}$ or with a beam of flux level $7 \mathrm{j} \mathrm{cm}^{-2} \mathrm{~ns}^{-1}$ only at temperatures about $130,000^{\circ} \mathrm{K}$. Since these temperatures are well above the range where damage is expected, and since radiation losses from the particles vary as $T^{4}$, such losses can well be neglected in cases of practical interest.

Temperature profiles into the glass adjacent to Pt particles of various sizes are shown in Figs. 4, 5 for various conditions of pulse time and flux level [calculated from eq (2)]. As shown there, a volume of hot glass surrounding the inclusion particles is expected in all cases. The extent of this hot layer is generally of the order of $\left(D_{g} t\right)^{\frac{1}{2}}$, and is most significant in the case of small
particle sizes.

In the range of large particle sizes, where $D_{i} t<R_{i}$, the assumption of a perfectly-conducting inclusion particle becomes inappropriate, and the 'ilternative model of the infinite composite solid may usefully be employed. The transition form small-particle behavior described by eq (1) to largeparticle behavior, described by eq (3), should shift to larger particle sizes for pulses of longer duration. The size range of this transition is about $1000 \AA$ for a 0.3 ns pulse, and about $10 \mu$ for a 3 us pulse.

For particle sizes.in the range where eq (3) is appropriate, the temperature at the particleglass boundary is expected to increase as $\mathrm{Jt} \frac{1}{2}$, and hence should be higher for high flux, short time pulses than for small flux, long time pulses of a given energy density. The temperature decreases 
less rapidly with distance into the particle than with distance into the glass (a difference associated with differences in the thermal conductivity of the two materials). Examples of the surface temperatures (at the inclusion-glass boundaries) expected for various pulse times and energy densities are shown in Fig. 6 .

\section{Stresses}

The view has been adopted (4) that failure of the glass will result when the tensile stress produced by the heating of inclusion particles relative to the surrounding glass exceeds the intrinsic strength of the glass. For typical glasses, this would correspond to a tensile stress of 500,000 psi or more. To introduce an element of safety into our calculations, however, we shall consider stresses exceeding $200,000 \mathrm{psi}$ as dangerous and smaller stresses as safe.1

In estimating the maximum tensile stress in the glass, jt is important under many conditions - particularly when $R_{i}<D_{g} t$ - to consider explicitly the layers of molten glass adfacent to the inclusion particles. The extent of such layers can be estimated from the temperature distributions calculated from eq (2), using the notion that solid-like behavior may be expected when the time scale for molecular rearrangement becomes longer than the experimental time scale. Using the Maxwell relaxation time as a measure of the rearrangement time, and taking the pulse time as a measure of the experimental scale, the transition from liquid-1ike to solid-1ike behavior may be expected when:

\section{$T_{\text {Manele }} \approx t_{\text {PULSE }}$}

or

$$
\eta \approx M t_{P U L S E}
$$

where $M$ is the appropriate modulus and $n$ is the corresponding viscosity. Taking $M$ as about 3 x 10 " dynes $\mathrm{cm}^{-2}$, the viscosity at the liquid-solid glass boundary is estimated as about $10^{2}$ poise for a 0.3 ns pulse, $10^{4}$ poise for a $30 \mathrm{~ns}$ pulse and $10^{6}$ poise for a $3 \mu \mathrm{s}$ pulse. For typical laser-base glasses, these viscosities correspond to temperatures in the range from about 1400 to $600^{\circ} \mathrm{C}$; and in the following discussion a value of $1000^{\circ} \mathrm{C}$ will be assumed as the temperature of the liquid-solid glass boundary.

In previous calculations (4), an approximate analysis was adopted in estimating the maximum tensile stresses expected for various particle sizes and conditions of laser operation. The average temperature of the particle + liquid glass volume was evaluated by neglecting the heat external to the solid-1iquid glass boundary; and a solution was used for the problem of a sphere of size $R+\Delta R$ in a hole of size $R$ in an infinite elastic solid. This solution neglects the effect of the temperature rise in the external glass diminishing the tensile stress component in the glass. This last could be justified, at least approximately, by noting that the temperature in the external glass is much less than the average temperature of the particle + liquid glass volume. Finally, the heat capacities and thermal expansion coefficients of inclusion and glass were taken as equal; and a bulk modulus of the particle + 1iquid glass volume intermediate between that of $\mathrm{Pr}$ and glass was assumed.

In the present paper, we shall adopt a less approximate treatment of the stress problem by integrating the calculated temperature distribution, given by eqs ( 1 ) and (2), out to the desired radius to obtain the average temperature of material inside that radius. This will be combined with a solution to the thermal stress problem (7) for a material with a radial temperature distribution of arbitrary form which includes the effect of the temperature in the glass external to the solid-1iquid glass boundary. This last effect may be visualized by recalling that a spherical hole in an infinite elastic solid will not change in size as the body is heated, but rather a compressive tangential stress will be established in the glass around the hole. In the present case, such a compressive stress compensates for at least a portion of the tensile tangential stress which is produced by placing an oversized particle in the hole.

Using the standard solution (7) to the thermal stress problem, the (tangential) tensile stress at a distance, $r$, from the center of a radial temperature distribution may be expressed:

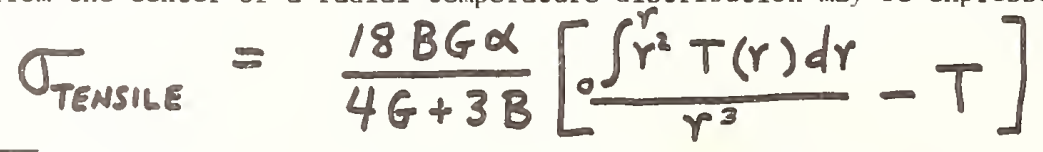

1 In our previous paper (4), we considered only stresses exceeding 600,000 psi as dangerous. The present estimate has been adopted to provide a margin of safety in the calculations and to compensate for expected increases in the spectral emissivity with temperature, which under the extreme conditions encountered in high power laser operation could significantly increase the temperatureand hence the stress - from values calculated using low temperature value for $\varepsilon_{\lambda}$. 
The first term corresponds to the expression obtained previously, and the second term corrects for the temperature external to $r$.

Evaluating this expression for various combinations of particle size and conditions of laser operation, the results shown in Figs. 7-9 are obtained. The first shows stress profiles at various distances out from the solid-liquid glass boundary for a $5000 \AA$ Pt particle subject to laser pulses of various durations. As seen in the figure, the maximum tensile stress in general occurs not at the solid-liquid glass boundary but at some distance into the glass. This results from the opposition of the two terms in eq (6). Further, the stress immediately adjacent to the boundary can under some conditions be compressive in character (represented by the negative sign in Fig. 7).

The variation of the maximum tensile stress with particle size for a $0.7 \mathrm{fm}^{-2} \mathrm{~ns}^{-1}, 30 \mathrm{~ns}$ pulse is shown in Fig. 8. As seen there, the maximum tensile stress should be sufficient to result in failure for a range of particle sizes from about $900 \AA$ to $0.9 \mu$. The maximum stresses for various combinations of flux level and pulse time having a given energy density are shown in Fig. 9. As shown there, the stress behavior is similar in form to the thermal behavior shown in Fig. 2 above; like the temperature, for a small particle size the stress produced by a short time, high flux pulse of a given energy density can be higher than that produced by a longer time, smaller flux pulse of a higher energy density.

Since each of the terms in eq (6) is proportional to the flux level, the maximum tensile stress for a given particle size should be linear in the flux; and hence the minimum size that is dangerous should decrease with increasing flux level. For example, for a 30 ns pulse time, increasing the flux level from 0.7 to $2.1 \mathrm{j} \mathrm{cm}^{-2} \mathrm{~ns}^{-1}$ decreases the minimum dangerous size from about $900 \AA$ to less than $500 \AA$

For a given pulse time and flux level, particles smaller than this minimum size are safe because of heat flow to the surrounding glass, while the very large particles are safe under the assumption of perfectly-conducting particles because of their large thermal mass. For such large particles, (R ${ }_{i}>$ $\left.D_{1} t\right)$, however, the effect of temperature gradients in the particles can be quite important; and as we have seen, the surface temepratures of such particles can be well into the $10^{4} \mathrm{O}_{\mathrm{K}}$ range under conditions where the average temperatures is appreciably lower. The precise magnitudes of the stress in this case difficult to evaluate because of the shape of the isothermal contours in the particles and a general uncertainty as to the appropriate equation of state for the particle material. From observations of extensive damage when large $\mathrm{Pt}$ particles are intentionally introduced into the glass (3) and from estimates of the expected interval pressure of the particles (4), it seems apparent that such particles must be regarded as dangerous under conditions of high power operation.

\section{Change of Phase: Pressure in Particles}

In the preceding calculations, as in the original work, we have neglected the effect of phase changes in the particle material. In the case of fusion, this assumption would not significantly affect the results since the heat absorbed on melting even under standard conditions corresponds to a temperature change of only about $800 \mathrm{~K}$, and hence from the thermal expansion coefficient a volume change of only about $2.2 \%$, while the volume change in fusion is about $3 \%$.

In the case of vaporization, the considerations are more complex. First, it must be recalled that the particles are confined by the glass, and very large superheatings above the standard boiling point would be required to form the vapor phase (4). Further, boiling of the confired particle material can only take place to a very dense gas with a density comparable to that of the liquid; and small or negligible differences in bulk properties are therefore expected between liquid and vapor. For these reasons, it seems sensible to neglect any absorption of heat by vaporization; and the calculations of particle temperature and stress in the preceding sections should provide reasonable estimates of these quantities.

These suggestions are reinforced by recent work (8) which indicates that as the confined inclusion particles are heated, they are highly unlikely to pass into a two-phase (liauid + vapor) region where vaporization can take place. Further, the new work indicates that even if vaporization of the superheated liquid did take place, it would only raise the pressure from the values estimated previously.

An alternate approach to estimating the tensile stresses in the glass adjacent to inclusion particles involves an estimation of the internal pressure of the particles (or of the surface regions of particles). Arguments have been advanced (4) which indicate that particle pressures within a factor or two of the ideal gas pressures for the given temperature may be anticipated; and the magnitudes of these pressures are similar to the inclusion pressures calculated from the thermal expansion. These results were used to justify caution about the safety of large particles whose average temperatures are much lower than their surface temperatures. From the present discussion, the high surface temperatures would result in locally high pressures, and could well result in failure. The establishment of a precise breakdown condition for this case involves, however, the solution of complex heat flow and stress problems which lie beyond the intent of the present work. For purposes of the present work, therefore, all particles with sizes larger than the minimum dangerous size will be regarded as likely to cause failure. 


\section{Type of Inclusions}

The calculations of the preceding sections were addressed to the case of Pt particles in a typical laser glass. Similar reasoning and comparison of the thermal, optical and mechanical properties of various likely metals suggest that similar behavior may be expected for other metallic inclusions. Differences in detail - resulting from differences in the heat capacity, thermal expansion coefficient and spectral emissivity - may be anticipated; but the general conclusion seems clear, vis., that metallic particles, even small metallic particles, must be regarded as dangerous in high-power laser operation.

For this reason, it seems clear that laser glasses should be prepared as free as possible from elements which are likely to form metallic inclusions. This is a decidely non-trivial task, however, since concentrations of inclusion material in the p.p.b. range can lead to extensive damage, and since the inclusions may have their origin in the batch raw materials, including certain fining agents, or in the crucibles used to melt the glass.

Considering non-metallic inclusions, it should be noted that only those inclusions which absorb strongly at the wavelength of the laser beam are important. At the wavelength of a Nd 1 aser ( $1.06 \mu$ ), the absorption of a good glass host is in the range of a few tenths of a percent per cm.; and absorption at any such level can be neglectęd in treating inclusion damge. Among ions of potential significance for the present problem, only $\mathrm{Fe}^{2+}, \mathrm{Ni}^{2+}, \mathrm{Cu}^{2+}$ and $\mathrm{Co}^{2+}$ absorb appreciably at $1.06 \mu$. Among these, the strongest absorber is $\mathrm{Fe}^{2+}$; and the most highly absorbing ceramic inclusion would then be one of Fe0, while a more typical iron-rich inclusion might have an Fe concentration 1 ike that of $\mathrm{Fe}_{2} \mathrm{SiO}_{4} \mathrm{~F} \mathrm{For}_{\mathrm{k}}$ these cases, the absorption coefficients would be about $0.20 \mu^{-1}$ and $0.08 \mu-\mu^{2}$ respectively. With these values, the energy absorbed by a spherical particle may be obtained from the relation:

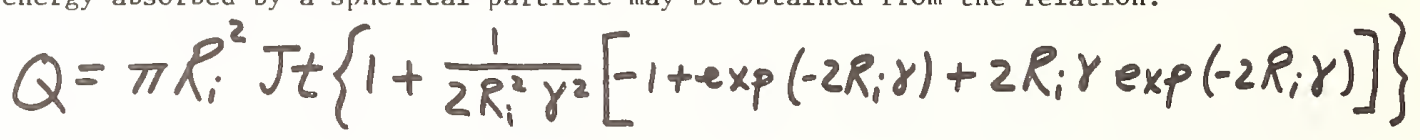

This expression leads to a mean particle temperature, neglecting heat flow into the glass, which increases with decreasing particle size and reaches a limiting value in the case of very small particle sizes. This limiting value is the same as the surface temperature of a ceramic inclusion when heat losses to the glass are neglected:

$$
\bar{T}=\frac{\gamma J t}{C_{v i}}
$$

For a $20 \mathrm{j} \mathrm{cm}^{-2}$ pulse, this expression indicates a temperature of about $10,000^{\circ} \mathrm{K}$ for $\mathrm{FeO}$ and about $4000^{\circ} \mathrm{K}$ for $\mathrm{Fe}_{2} \mathrm{SiO}_{4}$. Since the thermal expansivities of these and similar oxides are in the same range as that of $\mathrm{Pt}^{2}$, while their moduli are generally smaller (corresponding to a smaller stress in the glass), and since the temperatures estimated above for the most dangerous composition are no higher than these which correspond to the dividing line between safe and dangerous behavior, and since heat flow into the glass has been neglected and its introduction would decrease the estimated inclusion temperature and the resulting stress, it is concluded that ceramic inclusions would not be dangerous at the $20 \mathrm{j} \mathrm{cm}^{-2}$ level.

At higher power levels - as in the range of $50 \mathrm{j} \mathrm{cm}^{-2}$ and above - some such ceramic inclusions may result in failure. The likelihood of this damage can, of course, be reduced by reducing the concentrations of the highly-absorbing transition metal irons ( $\mathrm{Fe}^{2+}, \mathrm{Cu}^{2}+$ and $\mathrm{Ni}^{2+}$ ) in the melt, and by carryout the melting so as to promote complete dissolution. Beyond this, it is clear that ceramic inclusions, even those containing large concentrations of highly absorbing ions, are far less dangerous than metallic inclusions; and the large part of technical concern in this area should be directed to the latter.

\section{Particle Shape}

The discussion of the preceding sections has been concerned with spherical particles. In proceeding to evaluate the effect of particle shape on the conclusions, we shall consider cylinders of a given volume having various length-to-diameter ratios. This ratio will be denoted as a with large a values corresponding to needles and small a values to plates. The orientation of the cylinder axis to the beam direction will be denoted as $\theta$; and the projected area of the cylinder normal to the beam will be designated $A \theta^{\circ} \quad$ Neglecting heat loss to the glass, the temperature of a perfectly conducting particle characterizèd by an angle $\theta$ may be written:

$$
T=\frac{\varepsilon_{\lambda} J \hat{C} A \theta}{V C_{V i}}
$$

where $V$ is the volume of the particle.

Evaluating $A_{\theta}$ from the geometry of a cylinder, the temperature of a cylinder of orientation $\theta$, relative to the temperature of an insulated sphere of the same volume, may be written: 


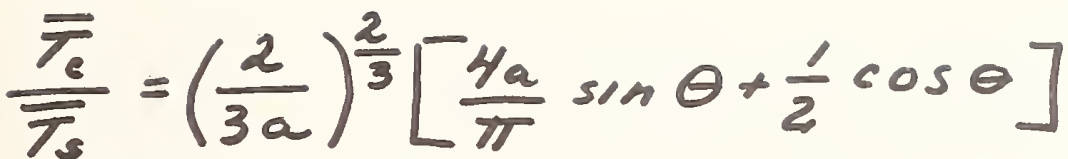

This is shown in Fig. 10 for various length-to-diameber ratios. As shown there, for such ratios of less than 10 to 1 (or 1 to 10), the maximum temperature of the cylinder is within a factor of two of that of the corresponding sphere. Only for much larger shape asymetrles does $T / T_{S}$ reach large values; but for such cases, the estimates of inclusion temperature based on an assumed spherical shape could be significantly in error.

Departures from spherical shape may then be important under some conditions; but in most cases the assumption of spherical particles should provide a reasonable estimate of the stress and hence of the likelihood of damage. Even in cases where large shape asymmetries are involved, the preceding analysis should prove useful since the stress resulting from a given change in volume, and hence from a given rise in temperature should be greatest for a sphere. Unfortunately, no data seem to be avallable on the distribution of particle sizes and shapes to be found in laser glasses, and nothing is known about the distribution of orientations of non-spherical particles (which is expected to vary with the processing techniques used to fabricate the glass).

\section{Discussion and Conclusions}

The analysis and discussion of the previous sections have identified the likely sources of inclusion damage in laser glasses, and have established conditions on particle size and material parameters which will lead to failure of the glass. In obtaining these results, the heat flow problem has been represented by two models: (a) the model of perfectly-conducting metal particles in good thermal contact with a medium having the thermal conductivity of a laser glass. This model should be appropriate for particles small with respect to the thermal diffusion distance in them $\left(R_{i}{ }^{2}<D_{i} t\right)$; and (b) the infinite composite solid, which should provide a useful estimate of the range of temperatures expected for particles large with respect to the thermal diffusion distance in them $\left(R_{i}{ }^{2}>D_{1} t\right)$.

The results of the thermal analysis indicate that the temperatures of metal particles subject to high energy laser pulses can exceed $10^{40} \mathrm{~K}$ for a range of particle sizes. Very small particles have lower temperatures because of heat losses to the surrounding glass, while large particles have low average temperatures because of their thermal masses. In the latter case, the surface regions of the particles are expected to be well in the $10^{4}$ degree range, even when the average particle temperatures are much lower.

In the limit of very small sizes $\left(R_{i}<\lambda / 10\right)$, the dependence of the effective absorption cross-section of the particles must be included, and would have the effect of decreasing the particle temperature and resulting stress. For a Nd laser, these effects should be significant below about $1000 \AA$ in size.

Phase changes in the particle material have been neglected in the thermal calculations. In the case of melting, the heat absorption on melting would be very nearly compensated by the accompanylng volume change, with little change expected in the estimated stress. In the case of vaporization, a number of arguments have been advanced: (1) Because of its confinement by the glass, any vaporization of the particle material would take place to a vapor which differs little from the liquid in density; and such a vapor should have bulk thermodynamic properties similar to those of the liquid; (2) extensive superheating of the particle material would be expected before the vapor forms; (3) the pressures of the vapor would, by a corresponding states analysis, not differ greatly from those estimated from the thermal expansion, and by recent analysis would be higher than those calculated neglecting vaporization; and (4) vaporization is highly unlikely to occur prior to failure since the heated and confined particle material should not pass into the two-phase $\mathrm{P}-\mathrm{V}$ region where vaporization is possible.

Combining the results of the thermal analysis with the solution to the stress problem for a material. with a radial temperature gradient, it is concluded that tensile stresses exceeding the expected strengths of glasses can be produced by high energy laser pulses. In treating the stress problem, consideration has been given to the occurrence of molten glass layers adjacent to the particles, and to the tangential compressive stress arising from the surrounding glass being heated.

The maximum tensile stress produced by laser heating of inclusion particles is expected not to occur at the particle-glass interface, nor at the solid-liquid glass boundary, but rather at some distance into the glass. This distance is of the order of a few times the glass thermal diffusion distance, (D $t)^{\frac{3}{2}}$, from the periphery of the inclusion particle.

The temperatures of small particles $\left(R_{,}<D_{1} t\right)$, as well as the resulting tensile stress in the glass, are expected to increase linearly with the and pulse times of tens of ns or larger, particles as small as $1000 \AA$ are expected to cause damage. Such particles would be exceedingly difficult to detect a priori, since their size is small and their presence in even small concentrations can result in unacceptable damage. 
For a given small particle size, the particle temperature and the maximum tensile stress in the glass can be higher for a short time, high flux pulse having a given energy density than for a longer time, smaller flux pulse having a larger energy density. This behavior indicates that damage testing a laser glass by subjecting it to a pulse of long duration can provide misleading information as to the safety of the glass under conditions of shorter pulse times. Long-time pulses are expected to cause extensive damage of the larger particles, but may provide an inadequate check on the presence of smaller particles which are dangerous with short-time, high flux pulses. For example, a $1500 \AA \mathrm{Pt}$ particle subfect to a $20 \mathrm{j} \mathrm{cm}^{-2}$, 30 ns pulse would result in damage to the glass, while the same particle subject to a $300 \mathrm{~ns}$ pulse with an energy density five times higher $\left(100 \mathrm{f} \mathrm{cm}^{-i}\right.$ ) would be safe.

The analysis has indicated that the particles of primary concern in causing inclusion damage are metallic. At energy densities in the range of $20 \mathrm{j} \mathrm{cm} 2$, ceramic inclusions are expected to be safeeven those containing large concentrations of high absorbing ions. At significantly higher energy densities, some of the latter particles may prove dangerous; but under all conditions such inclusions should be appreciably less dangerous than corresponding metal particles.

For shape asymmetries of 10 - 1 or less, the assumption of spherical inclusion particle should not introduce any significant error into the calculations. For larger shape asummetries, the geometrical shapes of the inclusion particles may well be significant in affecting the breakdown condition.

The other principal assumptions used in the analysis have been discussed previously (4), and indicate that the results should be good to better than order-of-magnitude accuracy; and the specification of the break-down condition should be useful as a guide to the range of particle sizes and the conditions of laser operation which must be regarded as dangerous. The difficulty of detecting a priori particles near the lower size limit for damage under high power operation leaves two apparent alternatives: (1) test individual samples of laser glass by subjecting them to pulses of somewhat higher beam intensity than the desired operating levels; or (2) adopt more stringent procedures to avoid the possible presence of metallic inclusions. For obvious reasons, the second alternative should be preferred.

\section{Acknowledgements}

The authors are happy to acknowledge stimulating discussion with Dr. E. Snitzer of the American Optical Company and Professor D. Turnbull of Harvard University. Financial support for the work was provided by the Advanced Research Projects Agency under Contract ARPA-SD-90, and by the American Optical Company, who provided one of the authors (R.W.H.) with the American Optical Fellowship in Materials Science. This support is gratefully acknowledged.

\section{References}

[1] E. Snitzer, Glass Ind. 48, 11 (1967).

[2] L.I. Van Torne, Phys. Stat. Sol. 16, $171,(1966)$.

[3] C.G. Young and R.F. Woodcock, in "Damage in Laser Glass", ASTM STP 469 (ASTM, Philadelphia, 1969).

[4] R.W. Hopper and D.R. Uhlmann, "On the Mechanism of Inclusion Damage in Laser Glass", J. App1. Phys., September, 1970.
[5] H.S. Bennett, National Bureau of Standards, to be published.

[6] M. Born and E. Wolf: "Princlples of Optics" (Pergamon Press, New York, 1959).

[7] B.A. Baley and J.H. Welner: "Theory of Thermal Stresses" (Wiley, New York, 1960).

[8] R.W. Hopper and D.R. Uhlmann: "On the Vaporization of Inclusions During Laser Operation": to be published. 
Symbols and Material Parameters Used in Text

I. Subscripts

$i$ = inclusion

$g=$ glass

II. Symbols

$\mathrm{k}=$ thermal conductivity

$D=$ thermal diffusivity $\left(=k^{\prime} / C_{m} \rho\right)$

$\rho=$ density

$\mathrm{C}_{\mathrm{v}}=$ heat capacity per unit volume

$\mathrm{R}=$ radius of inclusion

$\mathrm{J}=$ flux level

$t=$ time

$\varepsilon_{\lambda}=$ spectral emissivity

$\mathrm{T}_{\mathrm{M}}=$ melting point of inclusion

$\mathrm{T}_{\mathrm{B}}=$ boiling point of inclusion

$\alpha=$ linear thermal expansion coefficient

$\mathrm{V}=\mathrm{v}_{\mathrm{ol}} \mathrm{ume}$

$\mathrm{Q}=$ heat

$\mathrm{B}=$ bulk modulus

$G=$ shear modulus

$\sigma=$ stress

$L_{M}=$ latent heat of melting

$L_{B}=$ latent heat of evaporation

$Y=$ absorption coefficient

$\mathrm{C}_{\mathrm{m}}=$ heat capacity per unit mass
III. Values of Parameters

$\mathrm{k}_{i}=0.16 \mathrm{cal} \mathrm{cm}^{-1} \mathrm{sec}^{-1} \mathrm{o}_{\mathrm{K}}-1$

$\mathrm{D}_{i}=21.5 \mathrm{~cm}^{2} \mathrm{sec}^{-1}$

$\rho_{i}=21.5 \mathrm{~g} \mathrm{~cm}^{-3}$

$C_{v i}=0.66 \mathrm{cal} \mathrm{cm}^{-3} \mathrm{o}_{\mathrm{K}^{-1}}$

$\varepsilon_{\lambda}=0.3$

$\mathrm{T}_{\mathrm{M}}=2042^{\circ} \mathrm{K}$

$\mathrm{T}_{\mathrm{B}}=4100^{\circ} \mathrm{K}$

$\alpha_{i}=8.9 \times 10^{-6} o_{K}-1$

$\mathrm{B}_{i}=32 \times 10^{6} \mathrm{psi}$

$G_{i}=8 \times 10^{6} \mathrm{psi}$

$\mathrm{L}_{\mathrm{Mi}}^{i}=5 \times 10^{3} \mathrm{cal} \mathrm{mole} \mathrm{e}^{-1}$

$\left(\frac{\Delta V}{V}\right)_{M}=0.03$

$\mathrm{k}_{\mathrm{g}}=3 \times 10^{-3} \mathrm{cal}^{-1} \mathrm{cec}^{-1} \mathrm{o}_{\mathrm{K}}{ }^{-1}$

$\mathrm{D}_{\mathrm{g}}^{\mathrm{g}}=3 \times 10^{-3} \mathrm{~cm}^{2} \mathrm{sec}^{-1}$

$\rho_{\mathrm{g}}^{\mathrm{g}}=3 \mathrm{~g} \mathrm{~cm}^{-3}$

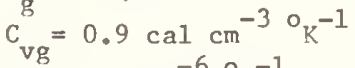

$\alpha_{\mathrm{g}}=9 \times 10^{-6} \mathrm{o}_{\mathrm{K}}-1$

$\mathrm{B}_{\mathrm{g}}=6 \times 10^{6} \mathrm{psi}$

$G_{g}^{g}=4.3 \times 10^{6}$ psi

$\mathrm{B}_{\text {PARTICLE + LIQUID GLASS }}^{\mathrm{g}}=15 \times 10^{6} \mathrm{psi}$

$\mathrm{C}_{\mathrm{mi}}=0.031 \mathrm{cal} \mathrm{g}^{-1 \mathrm{o}_{\mathrm{K}}} \mathrm{c}^{-1}$

$\mathrm{L}_{\mathrm{Bi}}=133 \times 10^{3} \mathrm{cal} \mathrm{mole}$

$\mathrm{C}_{\mathrm{mg}}^{\mathrm{Bi}}=0.3 \mathrm{cal} \mathrm{g}^{-1} \mathrm{o}_{\mathrm{K}}^{-1}$

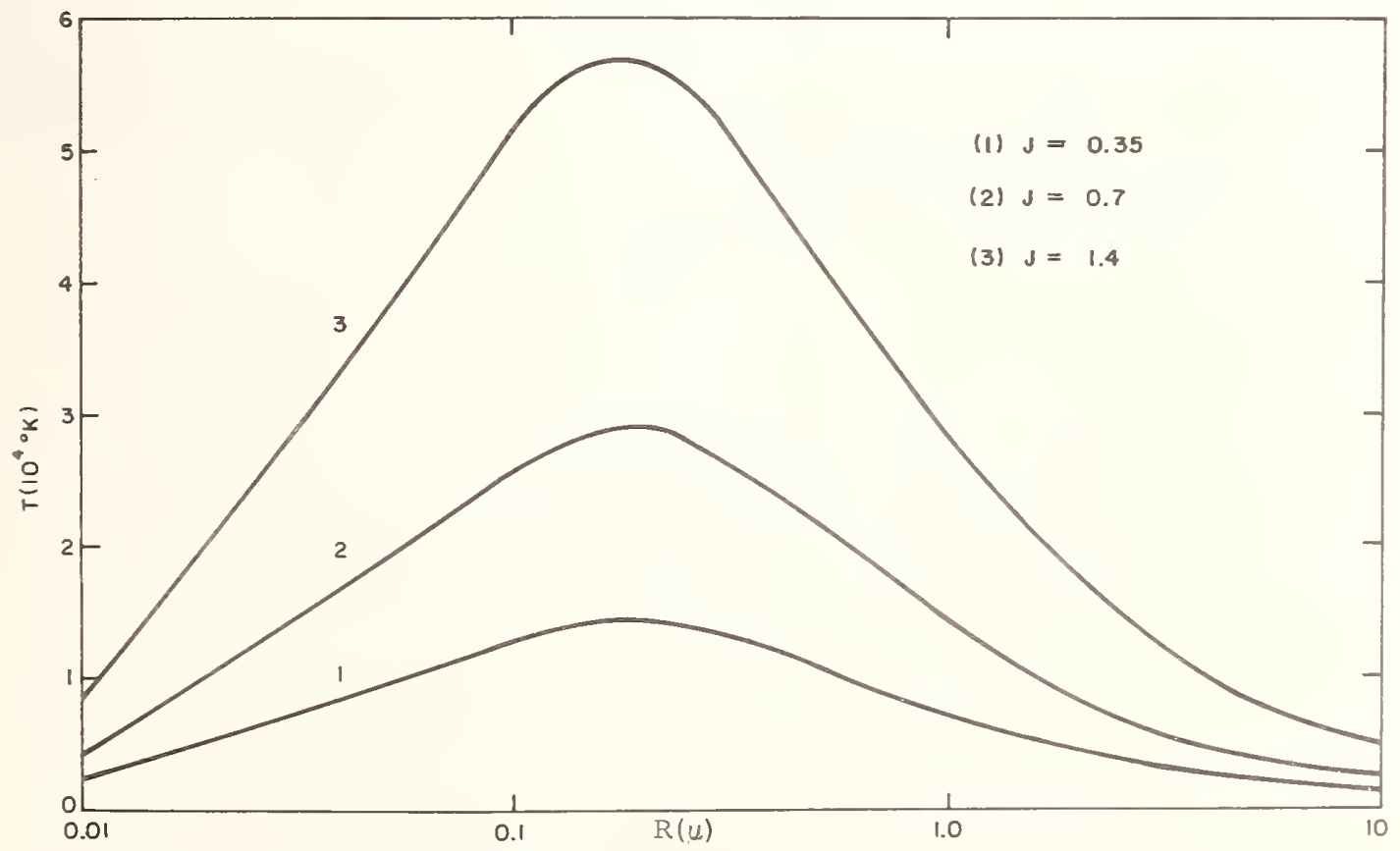

Fig. 1 Calculated temperatures of perfectly conducting platinum particles in a glass with thermal diffusivity, $3 \times 10^{-3} \mathrm{~cm}^{2} \mathrm{sec}^{-1}$, subject to 30 ns laser pulses. Various flux levels shown in $\mathrm{j} \mathrm{cm}^{-2} \mathrm{~ns}^{-1}$ 


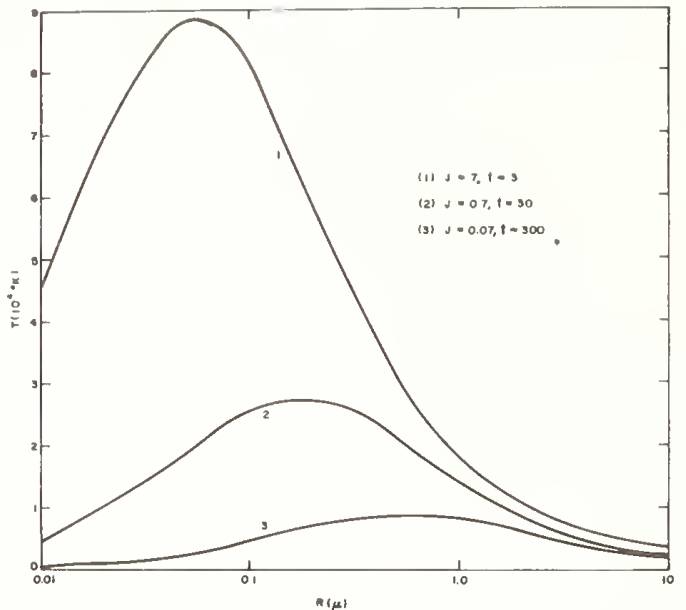

Fig. 2 Calculated temperatures of perfectly conducting platinum particles in a glass with thermal diffusivity, $3 \times 10^{-3} \mathrm{~cm}^{2} \mathrm{sec}^{-1}$, subject to laser pulses with energy density $21 \mathrm{j} \mathrm{cm}^{-2}$. Various combinations of flux level and pulse time shown.

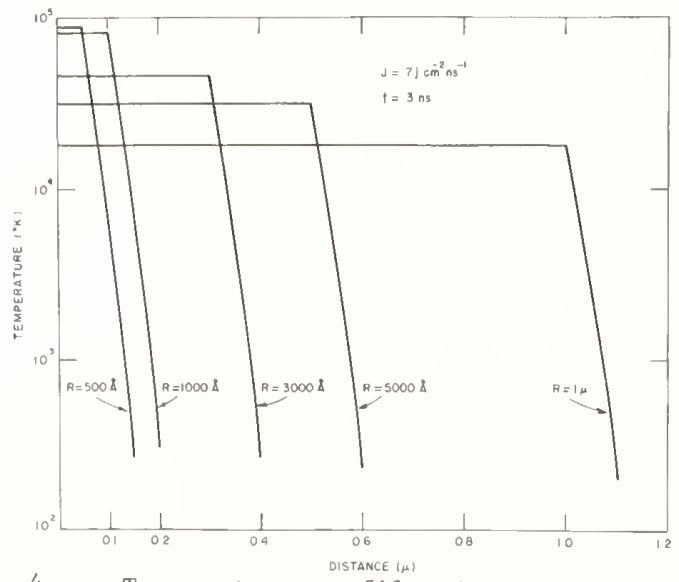

Fig. 4 Temperature profiles into the glass adjacent to platinum particles of various sizes subject to $21 \mathrm{j} \mathrm{cm}^{-2}$, 3 ns pulse.

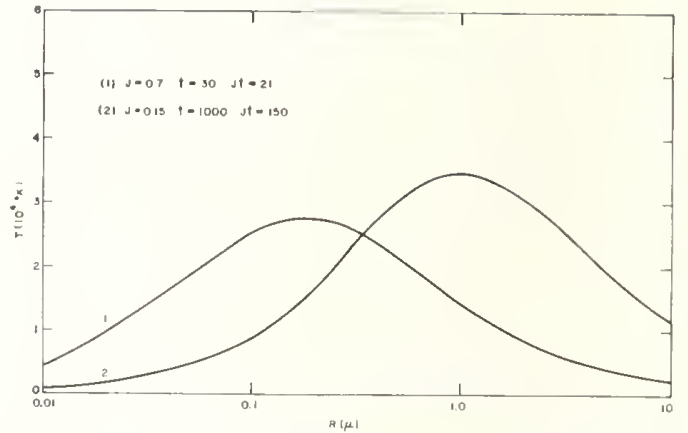

Fig. 3 Calculated temperatures of perfectly conducting platinum particles in a glass with thermal diffusivity, $3 \times 10^{-3} \mathrm{~cm}^{2} \mathrm{sec}^{-1}$ subject to laser pulses of $21 \mathrm{j} \mathrm{cm}^{-2}, 30 \mathrm{~ns}$ and $150 \mathrm{j}$ $\mathrm{cm}^{-2}, 1000 \mathrm{~ns}$.

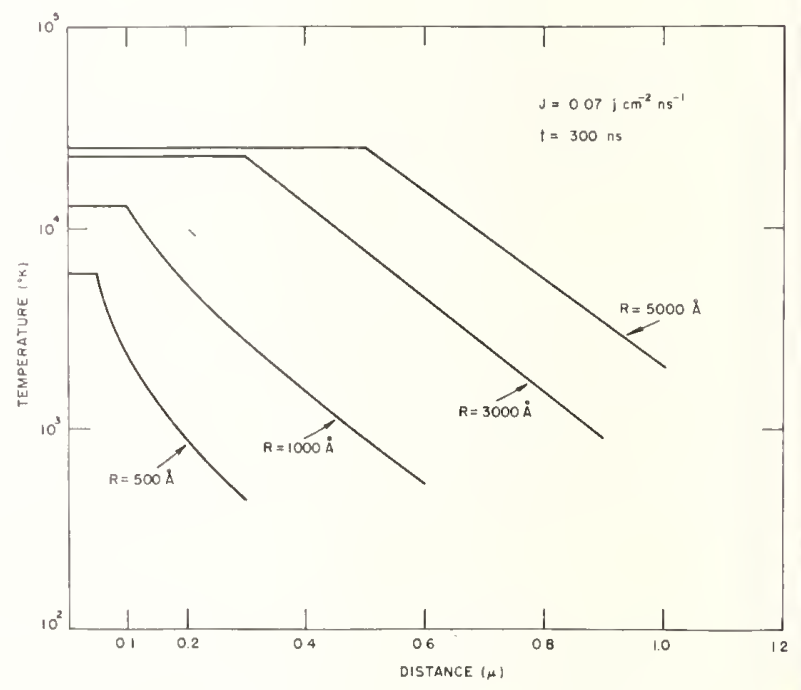

Fig. 5 Temperature profiles into the glass adjacent to platinum particles of various sizes subject to a $21 \mathrm{j} \mathrm{cm}^{-2}$, 300 ns pulse.

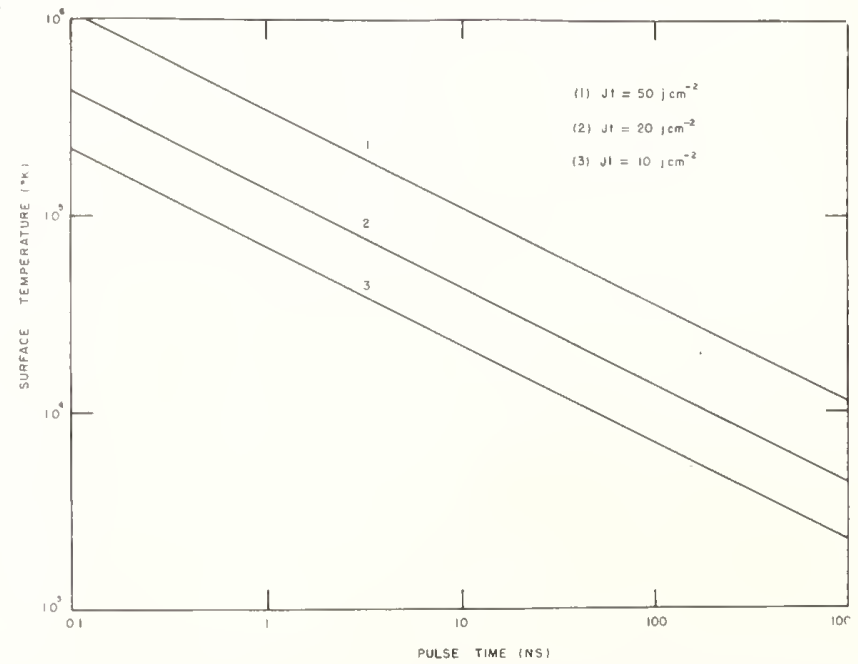

Fig. 6 Calculated surface temperatures of semi-infinite platinum particles in contact with semiinfinite glass slabs subject to laser pulses of various energy densities and pulse times. 


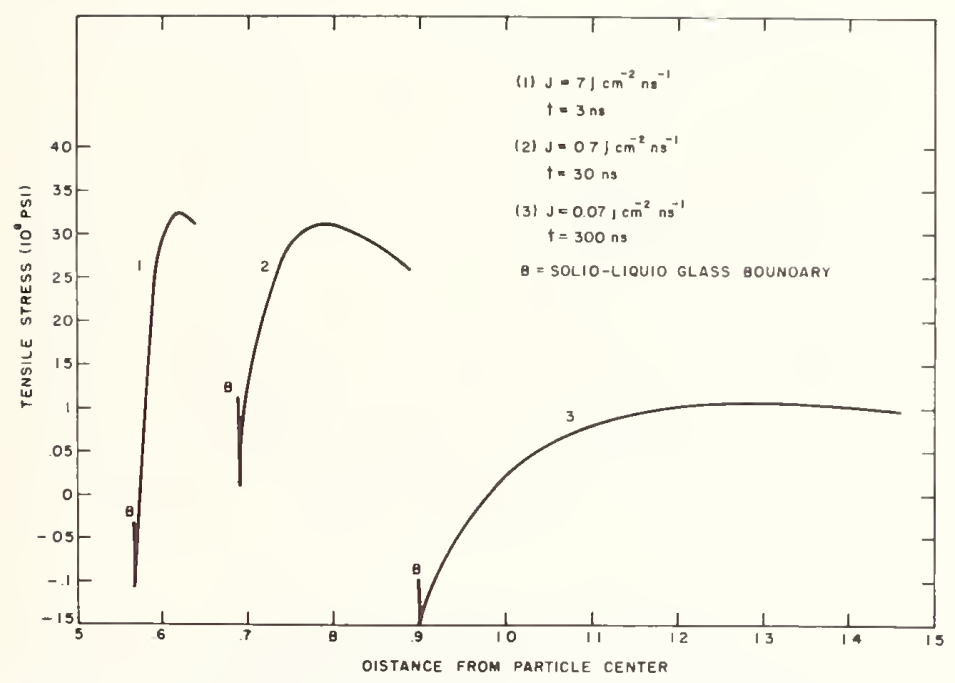

Fig. 7 Stress profiles into the glass from the solid-1iquid glass boundry surrounding a $5000 \AA$ platinum particle subject to laser pulses of various duration.

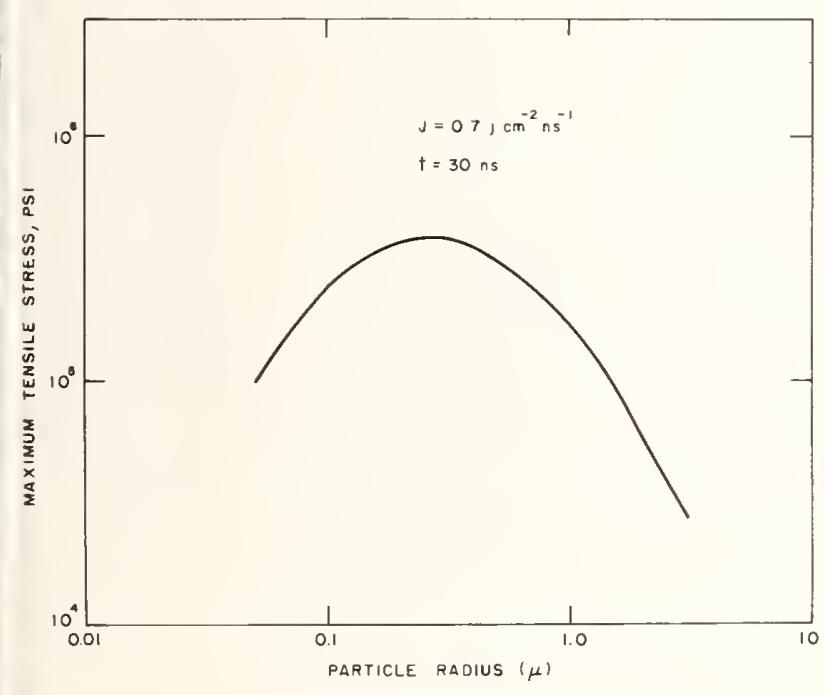

Fig. 8 Maximum tensile stress in the glass vs. particle size for platinum particles subject to a $21 \mathrm{j} \mathrm{cm}^{-2}$, 30 ns pulse.

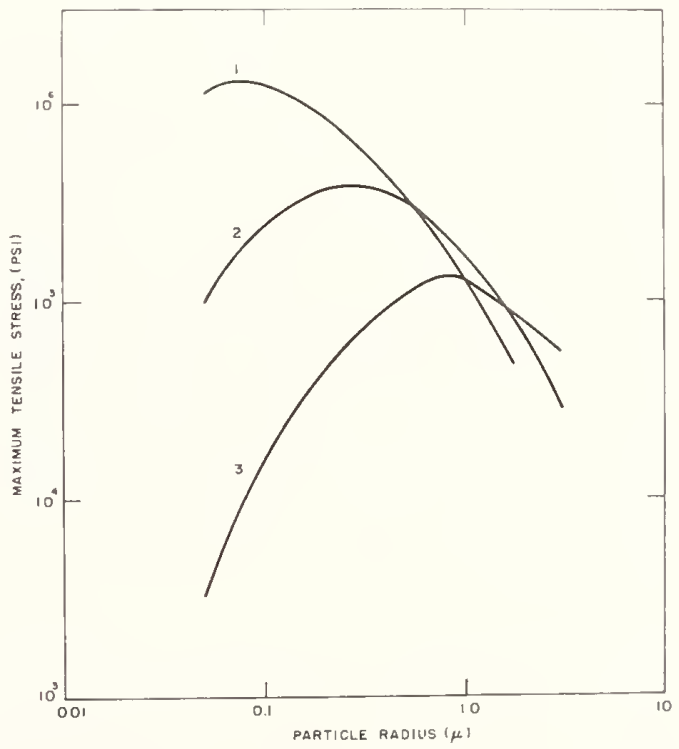

Fig. 9 Maximum tensile stress in the glass vs. particle size for platinum varticles subject to $21 \mathrm{j} \mathrm{cm}^{-2}$ laser pulses of various duration. $1=3 \mathrm{~ns}$; $2=30 \mathrm{~ns} ; 3=300$ ns. 


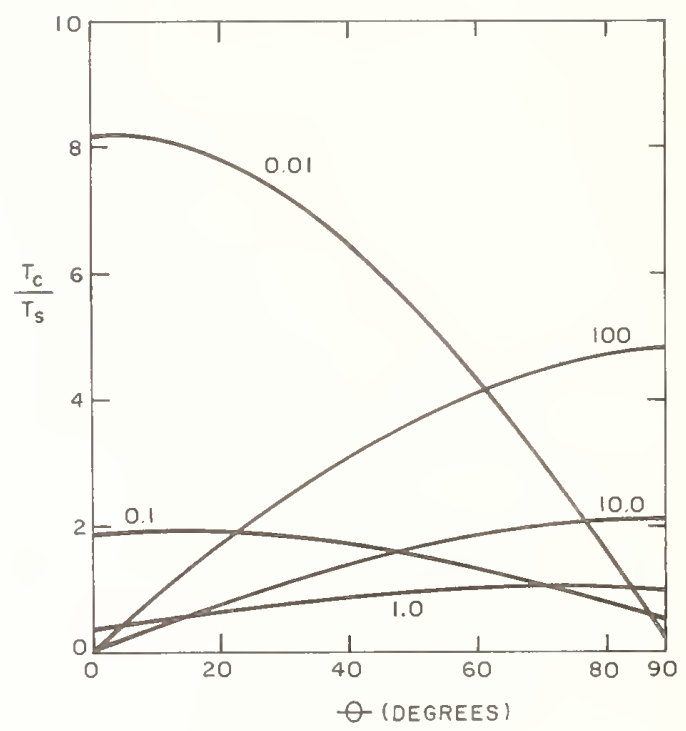

Fig. 10 Ratio of the average temperature of a insulated cylinder to that of an insulated sphere of the same volume as a function of orientation various length-to-diameter ratios.

\section{COMMENTS ON PAPERS BY HERBERT BENNETT AND ROBERT HOPPER}

Holographic investigations of particulate inclusions can be very fruitful, ifjone can resolve particles of the order of one $\mu \mathrm{m}$ in size. Although this has yet to be achieved, absorption of radiation of these inclusion, and consequent heating of surrounding naterial introduces a thermal distortion probably observable with present holographic sensitivity. Furthermore, it is not necessary to observe this distortion using pulse lengths characteristic of 2-spoiled operation, sunce it can be as easily observed using millisecand duration pulses. There is order of magnitude agrement between holographic observations and theoretical predictions of fringe shifts.

It was also suggested that Brillown scattering data, taken in conjunction with the predictions of the Landau-Placzek theory, may provide a useful technique for detecting the presence of particulate inclusions.

The theoretical treatment used in both these papers is invalid in the picosecond pulse range, due to the use of equilibrium assumptions. Furthermore, while the constraining forces of the surrounding medium were considered las in Seitz and Turnbull, Vol. 3) no inertial effects were included. The assumption of stress equilibrium across the interface is invalid for times like a nanosecond.

A further limitation imposed in the calculation was the use of geometrical cross-sections rather than the optical cross-sections predicted by the Mie theory. The latter effect is expected to be negligible for particles comparable to or larger than the wavelength.

Different boundary conditions were used in the two previous papers. In Dr. Bennett's calculation, an adhesive boundary was assumed, in which the strain and the radial component of the stress are continuous across the boundary, and no constraint is placed on the tangential stress. In the model of Hopper and Uheman, and assumption is made of a strain discontinuity at the boundary, with continuous normal stress components. 
Role of Photo-Electrons in Optical Damage ${ }^{1}$

R. W. Hellwarth

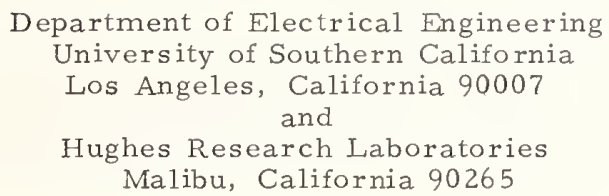

Previous workers have discussed the possibility that electrons photoexcited into the conduction band of a polar crystal by a high-intensity, short optical pulse may gain enough energy to damage the lattice by initiating an ionizing avalanche. We discuss here other processes by which these conduction electrons may damage the lattice even before they gain enough energy to ionize their surroundings, specializing our calculations to the case of sapphire and ruby. First, we show that the energy that the conduction electrons absorb linearly from the optical beam is deposited almost immediately in the lattice, without significant heating of the electrons. At electron densities $\left(\sim 10^{16} \mathrm{~cm}^{-3}\right)$ and optical intensities $\left(\sim 1010 \mathrm{~W} / \mathrm{cm}^{2}\right)$ likely to exist at sapphire damage thresholds, this deposited energy is found to be of the order of what one might expect would be required to form a rupturing shock wave. We also show that the photo-excitation of both bound and unbound impurity levels is enhanced by the presence of conduction electrons, so markedly so for the former that the promotion of electrons into the conduction band may be significantly "bootstrapped," thereby increasing the optical absorption. The presence of conduction electrons and excited impurities in the expected numbers is likely to alter the refractive index significantly and affect thereby the focusing (self- or external) of the beam in a complicated way. For the simplest model these nonlinear index contributions would tend to produce repeated focal regions along the beam. Implications of these results for raising damage thresholds are discussed.

\begin{abstract}
Key words: Crystals, electrons, optical damage, photo-absorption, photo-conductivity, photo-electrons, polar crystals, ruby, sapphire, self-focusing.
\end{abstract}

\title{
1. Introduction
}

The physical processes responsible for the bulk damage caused in various transparent crystals by short optical pulses (causing negligible electrostriction) have not yet all been identified. Here we argue that several processes not consider ed previously are likely to be important in the optical damage of inclusion-free polar crystals, especially sapphire and ruby. For their initiation, these processes would all seem to require on the order of 1016 conduction electrons per cc to be present at the point of maximum optical intensity, a number widely suspected to be present in sapphire and ruby at peak intensities $\sim 1010 \mathrm{~W} / \mathrm{cm}^{2}$ just below damage threshold. We will argue here that such densities of cold electrons can a) transfer damaging amounts of heat from the beam to the lattice; b) accelerate the rates of photo-excitation of bound and free impurity electron states, thus significantly increasing the supply of conduction electrons, possibly even in "avalanche"; and c) significantly change the local refractive index, possibly in a way which, in conjunction with the normal nonlinear index, could cause repeated beam focusing along its axis. In none of these processes do the electrons become hot enough $(\sim 8 \mathrm{eV})$ to ionize the lattice, in contradistinction to the process suggested by previous workers $[1-4]^{2}$.

${ }^{1}$ Work supported in part by the Joint Services Electronics Program (U. S. Army, Navy, and Air Force), under Grant No. AF-AFOSR-69-1622A, and in part by the Advanced Research Projects Agency through Air Force Cambridge Research Laboratories.

Figures in brackets indicate the literature references at the end of this paper. 
Hence, we will refer to them as "cold" conduction electrons. How the damage processes discussed here depend on crystal temperature, optical wavelength and pulse duration is a complex function of the energies and wavefunctions of the states of the impurities supplying and trapping conduction electrons, the electrons' effective mass(es), the longitudinal optical (LO) phonon structure, and the electronphonon coupling. Although only the latter two are fairly well known for ruby and sapphire, plausible hypotheses about the former lead one to single out the processes we propose as potentially important to damage. It would appear that reducing donor concentrations or increasing their electron binding energies might produce more damage-resistant crystals. However, if the conduction electron density were proportional to optical intensity (which appears not to be the case at such high intensities), it would tend to reduce self-focusing (or produce a net defocusing) and ther eby lower damage thresholds. Other conclusions and suggested experimental checks of electron processes are mentioned in the final Section. In the following Section 2 we summarize the parameters and physical regimes we encounter in ruby and sapphire and we outline our rough criteria for when damage is expected. In Section 3 we define the approach we take for evaluating the required parameters and discuss difficulties with previous calculations of the conditions under which hot ionizing electrons might be produced. The calculations of the enhancement by conduction electrons of lattice heating and photo-excitation follow in Sections 4 and 5. Before proceeding, we first summarize our assessment of often-conflicting reports on photoconductivity in ruby and sapphire.

Hochuli [5] has measured the low-frequency photoconductivity at various wavelengths and temper atures of ruby and sapphire at optical intensities I $\sim 0.2$ to $5 \mathrm{~W} / \mathrm{cm}^{2}$ and using applied voltages at frequencies zero $\mathrm{Hz}, 100 \mathrm{~Hz}$, and $9.39 \mathrm{GHz}$. He found no essential differences in results for the two crystals. The observed conductivities (at $5 \mathrm{~W} / \mathrm{cm}^{2}$ ) were $4 \times 10^{-13}, 10-11$, and $4.2 \mathrm{x} 10-8(\Omega \mathrm{m})-1$, respectively, at the above frequencies. Since all these frequencies are well below the electron collision frequency (which from the theory of Section 4 we estimate to be 1014 rps), the wide variations in results indicate the magnitude of experimental pitfalls in any attempt to measure such low bulk conductivities. Anomalies at the two lower frequencies probably arise from surface effects, from the inability of electric contacts to inject charges into the crystal, and from space charge buildup. Therefore, the $9.39 \mathrm{GHz}$ value, obtained in a microwave cavity with a small electron drift excursion amplitude (of the order of an Angstrom), is probably the most reliable. This conductivity was linear in the optical intensity within the range of observations. Its wavelength dependence suggested that the electrons were supplied from donor levels between 0.6 and $1.2 \mathrm{eV}$ below the conduction band, in agreement with what one would conclude by studying the normal bulk conductivity variation with temperature at higher temperatures. The wavelength dependence also suggested more donor levels appearing around $3 \mathrm{eV}$. Although Hochuli was able to observe a Hall voltage, it did not vary when the light was turned on or off. The nominal Hall mobility value $\mu=0.052 \mathrm{~cm} 2 / \mathrm{V}$ sec. derived therefrom has nevertheless been used without question by other workers whenever a value was required in calculations. This value corresponds to a collision time $T$ of $4 \times 10^{-17}$ sec., or an electron mean path of the order $10^{-10} \mathrm{~cm}$, and must be considered unphysical, corroborating the difficulty of making electrodes on sapphire. Using the standard Frohlich theory of a conduction electron in a polar crystal adapted to the large coupling constant of sapphire [6], one estimates $T$ (at room temperature) to be between $10^{-14.4}$ and $10^{-13.4} \mathrm{sec}$, depending on where the effective band mass lies between $1 / 10$ and 10 electron masses. Assuming a free electron mass for which $T=7 \times 10^{-15} \mathrm{sec}(\mathrm{and} \mu=12 \mathrm{~cm} 2 / \mathrm{V} \mathrm{sec}$ ), one would infer from Hochuli's microwave observations that he produced $2 \times 10^{8}$ photo-electrons per $\mathrm{cc}$ with $5 \mathrm{~W} / \mathrm{cm}^{2}$ of broadband $\mathrm{Hg}$ lamp exciting intensity. We see from this that 1016 electrons per cc would very likely be excited near damage thresholds of $1010 \mathrm{~W} / \mathrm{cm}^{2}$ provided that 1016 donor levels per cc were occupied, a provision which is unknown.

Except possibly for the experiments of Belikova, et al. [7,8], other reported observations of photoconductivity in ruby appear to be plagued by surface and contact effects. Belikova, et al. , observed the photoconductivity to be highly nonlinear with the $(6943 \AA)$ optical intensity I for intensities near $1010 \mathrm{~W} / \mathrm{cm}^{2}[7]$. One can use our value of $\tau$ above with their data to estimate very crudely (since their beam geometry was unspecified) that they were observing well over $10^{16}$ electrons/cc at $1010 \mathrm{~W} / \mathrm{cm}^{2}$ (provided that the electron recombination time was short compared with the optical pulse length). Belikova, et al., also observed optical emission bands near 2, 2. 7, and 3 eV (they could not see below $2 \mathrm{eV}$ ) which are suggestive of some impurity level transitions. The foregoing is about all one can conclude about photo-electrons from existing data for ruby and sapphire.

\section{Description of Electron Interactions}

In studying the role of photo-excited electrons in crystal damage, we will constantly apply our formulae to the case of sapphire and ruby. The phonon structure of these two crystals are essentially identical, as are the low-intensity photoconductivities. Although distinct differences in damage behavior have been observed, the gross damage thresholds are statistically indistinguishable for sapphire and ruby. Therefore, most pertinent aspects of these two crystals can be studied together. For brevity, we shall refer hereafter only to sapphire, but shall intend our remarks to apply also to ruby unless stated otherwise. We shall also assume throughout that the optical beam wavelength is $6943 \AA$, that of the room temperature ruby laser. 
A free electron gas of density $10^{16} \mathrm{~cm}^{-3}$ has a plasma frequency $\omega_{\mathrm{p}} \sim 6 \times 10^{12} \mathrm{rps}$ that is much lower than a) the optical beam frequency of interest $v\left(2.71 \times 10^{15} \mathrm{rps}\right)$, b) the phonon frequencies $\omega_{k}$ $\left(\sim 10^{14} \mathrm{rps}\right)$, and $\left.\mathrm{c}\right)$ typical electronic excitation frequencies ( 10l6 rps).

We shall call a conduction electron "cold" when its energy is much less than interband energies and its wavelength is much longer than the scale of potential variations of the field lattice. Other electrons we will refer to as "hot." The time for cold electrons in the $1 / 40$ to 3 volt range to equilibriate among each other by collisions at a density $10^{16} \mathrm{~cm}^{-3}$ is of the order of $10^{-10}$ to $10^{-13} \mathrm{sec}[9]$, much longer than the inverse electron-lattice collision time $\left(\sim 10^{-14} \mathrm{sec}\right.$ in ruby). Therefore, at least before any large ionizing avalanche occurs, the cold conduction electrons may be studied as independent particles, not interacting among themselves but interacting only with the fields and other particles in the crystal.

When a cold electron moves in a polar crystal such as ruby or sapphire, it is often called a "polaron." Its interactions with the crystal phonons are known to be well-described at room temperature and above by the Hamiltonian derived by Frohlich [10] and used by all previous workers in calculating electron motions leading to damage:

$$
\mathrm{H}_{\mathrm{F}}=\mathrm{h}_{\mathrm{O}}+\mathrm{v}_{\mathrm{F}}
$$

where

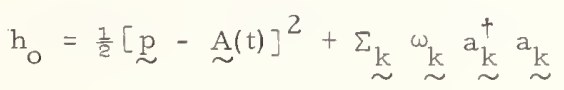

and

$$
\mathrm{v}_{\mathrm{F}}=\Sigma_{\mathrm{k}} \mathrm{C}_{\underset{\mathrm{k}}{\sim}} \underset{\sim}{\mathrm{a}_{\mathrm{k}}} \mathrm{e} \underset{\sim}{\mathrm{ik}} \cdot \underset{\sim}{\sim}+\mathrm{h} . \mathrm{c} .
$$

Here $\mathrm{p}$ is the electron's momentum, and $\underset{\sim}{\mathrm{x}}$ the electron's position coordinates, $\mathrm{a}_{\mathrm{k}}$ is the annihilation operation for a LO phonon of frequency $\omega_{k}$. Optical wavelengths are long enough so that the vector potential $\underset{\sim}{A}(t)$ may be taken as a function $\widetilde{\gamma}$ time only oscillating at a frequency $v$. We use dimensionless "polaron" units in which the effective band mass of the electron $m_{b}$ is unity as is Planck's constant n. All energies are measured in units of an effective $\mathrm{LO}$ phonon energy $\hbar \omega_{0}\left(840 \mathrm{~cm}^{-1}\right.$ or $1200^{\circ} \mathrm{K}$ for sapphire and ruby). Frequencies are measured in units of $\omega_{0}\left(=1.6 \times 10^{14} \mathrm{rps}\right)$ so that $v=17$ for a $6943 \AA$ beam in sapphire. Lengths are measured in units of $\left(\hbar / \mathrm{m}_{\mathrm{b}} \omega_{\mathrm{O}}\right)^{\frac{1}{2}}$ which is $8.5 \times 10-8 \mathrm{~cm}$ for ruby if we take $m_{b}$ to be the free electron mass $m_{0}$. Frohlich showed that in these units the coupling coefficients $C_{k}$ are well approximated by $V^{-1} 2^{3} / 4 l_{1}^{1 / 2} \mathrm{k}^{-1}$ where $\mathrm{V}$ is the crystal volume, a is the dimensionless polaron coupling constant $\left(\epsilon^{-1}-\epsilon_{0}^{-1}\right)\left(\text { Ryd }^{1} / \hbar \omega_{0}\right)^{\frac{1}{2}}$. Here Ryd' is the Rydberg for an electron with mass $\mathrm{m}_{\mathrm{O}}$, and $\epsilon$ and $\epsilon_{\mathrm{O}}$ are the optical and static dielectric constants (3.1 and 10). For $\mathrm{m}_{\mathrm{b}}=\mathrm{m}_{\mathrm{O}}, \mathrm{a} \sim 3$ for ruby. In ruby an electron is hot if $\mathrm{k}$ is of order 10 or more (in polaron units). Hot conduction electrons have neither the simple forms of kinetic energy or lattice interaction energies found in eq (1) but obey complex equations in which exchange must be accounted for and which have never satisfactorily been approximated in usable form for a dynamic lattice. There would seem to be no way at present to make a reliable estimate of how strong an optical field would be required to produce enough interband electronic transitions (i. e., lattice ionization) to cause damage.

From the discussion of the previous section, it is evident that the electrons photo-excited (below damage thresholds) in ruby and sapphire crystals come from donor impurities to which they are bound initially with much less energy than the valence-conduction band gap energy $(\sim 8 \mathrm{eV})$. The effects of thes e impurities on the supply and motions of conduction electrons, as well as on the excitation of the donors by conduction electrons, can be studied by considering the Coulomb interactions $v_{t}$ between a conduction electron at $\underset{\sim}{\sim}$ and the $i^{\text {th }}$ particle of the $a^{\text {th }}$ impurity having charge eq and located at $\stackrel{r}{\sim}_{i}^{a}$.

$$
v_{t}=e^{2} \epsilon_{s}^{-1} \sum_{i, a} q_{i}^{a} /\left|\underset{\sim}{\sim}-\underset{\sim}{r_{i}^{a}}\right|
$$

where the electronic charge $\mathrm{e}$ is 4 in our polaron units, and is screened by the dielectric constant $\varepsilon_{S}$ appropriate to the frequencies of motions under consideration.

To deal with the elastic scattering of the electrons, one needs only the matrix element of eq (3) diagonal in the impurity's ground state. This gives an effective classical scattering potential seen by the conduction electron. To study inelastic scattering, we will assume that the impurities may be described by an unperturbed. Hamiltonian $h_{t}=\Sigma_{a} h_{t a}$ with "ionized" or unbound as well as bound electronic eigenstates. Calculations will therefore start from a total Hamiltonian $\mathrm{H}$ comprised of 
Frohlich's Hamiltonian eq (1) (which describes cold electrons, phonons, and their interactions) plus the electron impurity interactions of eq (3) and with $h_{t}$ determining impurity states:

$$
H=h_{0}+h_{t}+v_{F}+v_{t} \text {. }
$$

We will assume that damage to the crystal occurs when the interactions $v_{F}$ and $v_{t}$ combined cause the conduction electrons to absorb an energy U per cubic centimeter with a short optical pulse that exceeds a threshold value $U_{0}$. That is, we shall strive to determine at what optical beam intensity we may expect

$$
\mathrm{U} \geq \mathrm{U}_{\mathrm{O}}
$$

Present evidence indicates that $U_{0}$ is of order $10^{2}$ to $10^{3} \mathrm{~J} / \mathrm{cm}^{3}$ for ruby and sapphire for $20 \mathrm{nsc}$ pulses in a beam of order one mm diameter. A shock wave of this energy surrounding the beam would develop stresses on the order of the static yield stress [3]. Obviously a large part of understanding optical damage in crystals entails developing an accurate theory of the amount of energy deposition required to produce rupturing strains as a function of the amount and spatial and temporal distribution of this deposition. However, we shall content ourselves here with the above crude estimate for U.

\section{Approaches to the Calculation of Damage Thresholds}

Previous treatments of the role of electrons in damage [1,4] started from Frohlich's Hamiltonian eq (1) and sought to calculate the optical intensity at which electrons would gain enough energy u to excite an avalanche of interband transitions. The expected energy of an electron in the optical field was estimated from the equation

$$
\frac{d u}{d t}=R_{f e}-R_{e l}
$$

where $R_{f e}$ is the average rate at which the optical field does work on the electron and $R_{e l}$ is the average rate at which the excited electron "radiates" phonons, i. e., transfers its energy to the lattice. Previous treatments have all taken for $R_{f}$ the usual linear conductivity loss per electron with reasonable order of magnitude estimates from eq (1) for the optical conductivity (or "electron collision rate' $)$. However, they have made approximations for, or statements about, $R_{e l}$ that are not directly based on a quantum average of the appropriate operator. Wasserman [1] took for $\mathrm{R}_{\mathrm{el}}$ the rate at which an electron having a Maxwellian distribution would lose energy to a cooler lattice in the absence of the optical field $(\mathrm{A}=0)$. He predicted breakdown intensities orders of magnitude above those actually observed. Sverev, et al. [4], used for $\mathrm{R}_{\mathrm{e}}$ the approximate rate at which an electron would lose energy to the lattice starting from a momentum eigenstate $|p\rangle$ for which $\frac{1}{2} p^{2}=u$ also in the absence of the optical field. His predicted thresholds also exceeded observed thresholds by over two orders of magnitude. We feel it is quite probable that a precise evaluation of the spectral energy distribution of an electron obeying eq (1) would show that the electrons do in fact remain too cool to ionize impurities or the lattic at optical fields below damage thresholds. In the next section we show that, indeed, collisions with phonons dominate an electron's motion. We have checked that it follows rigorously from eq (1) that, to lowest order in the optical intensity and coupling parameter a, an electron's expected kinetic energy is simply the sum of its coherently oscillating energy plus $3 \mathrm{kT} / 2$ where $\mathrm{T}$ is the ambient lattice temperature. The coherent energy is very much smaller than $\mathrm{kT}$ for optical intensities of interest. Therefore, we now proceed to examine whether cold electrons may not mediate the deposition of damaging amounts of energy in a sapphire lattice.

As we mentioned in the previous section, our approach will be to calculate the beam intensity at which the energy $U$ deposited per unit volume in the lattice from the optical field via the electrons exceeds a threshold value $U_{0}$. If the maximum optical beam energy per unit area is $S$, then

$$
\mathrm{U}=x_{v} \mathrm{~S}
$$

where $x_{v}$ is the absorption per unit length of an optical beam of frequency $v$. Following FHIP [6], we write the quantum expectation value of the amplitude of an electron's coordinate at frequency $v$ as Re Ee $e^{i v} /\left(-v^{2}+x_{v}\right)$ when the electron is in an optical electric field Re Eeivt. With this definition we have 


$$
x_{v} \lambda=\left(\omega_{p}^{2} / \varepsilon\right) \operatorname{Im}\left[v^{2}-x_{v}\right]^{-1}
$$

where $\lambda$ is the wavelength of the light in the crystal $\div 2 \pi$ and $\omega$ is the electron plasma frequency (in units of $\left.\omega_{\mathrm{o}}\right)$. In cgs units $\omega_{\mathrm{p}}^{2}=4 \pi^{\rho} \mathrm{e}^{2} / \mathrm{m}_{\mathrm{b}}$ with $\rho$ being the number density of electrons.

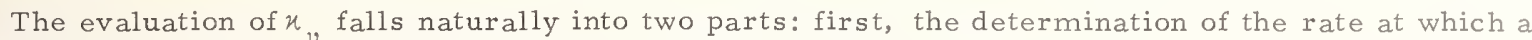
single conduction electron takes energy from the field (and delivers it to the lattice) by a calculation of $x_{y}$, and second, the estimation of the number density $p$ of these electrons. We consider these two questions, respectively, in the following two sections.

\section{Optical Response of Single Electron}

We examine first an electron's optical response in the case that it is affected only by electronphonon interactions as described by Frohlich's Hamiltonian eq (1) with coupling parameters appropriate to sapphire. We then find that, at least at room temperatures and above, collisions with impurities are relatively less important. When the electron response is linear in the applied field for a given lattice temperature, we may use the determination of $x_{v}$ by FHIP who calculated the quantum expected value of the electron position to first order in the optical field. Their expression is exact for all temperatures at small coupling $(a<1)$ and gives an accurate solution even when $a \sim 3$ as for ruby. In this case it shows that, for $v=17$, both the real and imaginary parts of $x_{v}$ are much less than $v^{2}$ so that we need only calculate $\operatorname{Im} x_{v}$ to estimate the absorption constant. (The $R e x_{v}$ can of course be determined from Im $x_{v}$ by Kramers-Kronig relations.)

$$
\operatorname{Im} x_{v}=\frac{2 a \pi^{-\frac{1}{2}} \beta^{3 / 2} \sinh (\beta v / 2)}{3 \sinh (\beta / 2)}\left(\frac{v}{w}\right)^{3} \int_{0}^{\infty} \frac{\cos (v u) \cos u d u}{\left[u^{2}+a^{2}-b \cos (v u)\right]^{3 / 2}} .
$$

Here $v$ and $w$ are parameters to be chosen from a variational principle. $a^{2} \equiv \beta^{2} / 4+R \beta \operatorname{coth}(\beta v / 2)$; $R \equiv\left(v^{2}-w^{2}\right) /\left(w^{2} v\right) ;$ and $b \equiv R \beta \sinh (\beta v / 2)$.

In the weak coupling limit $\mathrm{v}=\mathrm{w}=3, \mathrm{R}=\mathrm{b}=0$ and $\mathrm{a}=\beta / 2$. Then the integral can be evaluated exactly in terms of modified Bessel functions. At temperatures much lower than the Debye temperature $(\beta$ large) this result reduces to

$$
\operatorname{Im} x_{v} \rightarrow 2 a(v-1)^{\frac{1}{2}} / 3: a<1, \beta>>1, v>1 .
$$

For $a=3$ and $y=17$, this is 8 . The leading correction to eq (10) for finite temperatures multiplies it by $\left(1+\beta^{-1}\right)$, increasing it by $30 \%$ at room temperature $(\beta=3.3)$.

When $a=3$, the variational principle gives $v=3.4$ and $w=2.55$ in the zero temperature limit [6]. Using these parameters with $\beta=3.3$ gives $R=0.229, a=1.866$ and $b=5.53 \times 10-3$. We have evaluated eq (9) numerically for these parameters and have obtained $\operatorname{Im} \chi_{v}=15$. We have also evaluated eq (9) at frequencies in the neighborhood of $v=17$ and found that, as in eq (8), Im $x_{v}$ varies slowly with $v$. Therefore, electron transients as sociated with the rise and fall of even picosecond optical pulses are very small, and it is a good approximation in practice to as sume that at any instant the electron's motion is the same as for a purely sinusoidal field of amplitude appropriate to the intensity at that instant. The fast response of the electron's motion, as evidenced by the nonresonant character of $x_{v}$, together with the knowledge that, at intensities of interest, the electron is gaining negligible energy (staying "cold"), implies that the absorbed energy is being passed on essentially instantaneously to the phonons via the coupling eq (2b). Therefore, it is valid to obtain the rate of deposition of energy in the lattice by determining the $r a t e$ at which the electrons absorb energy from the optical beam, and we return to this problem.

When a room temperature correction is made to $v$ and $w$, we guess that, as for small $a$, the result is raised by around $30 \%$ so that the Frohlich model may be taken to yield

$$
\operatorname{Im} x_{v} \sim 20
$$

for a ruby laser beam driving an electron of band mass equal to a free electron mass in ruby or sapphire at room temperature. The main uncertainty, in eq (11) comes from the uncertainty in the effective band mass $m_{b}$. $\operatorname{Im} x_{v}$ varies roughly as $m_{b}^{1} / 2$. 
If the temperature goes higher than the phonon energy but is still small compared with $v$, then eq (9) yields

$$
\operatorname{Im} x_{v} \rightarrow 4 \alpha v^{1 / 2} /(3 \beta) \quad ; \quad \alpha<<1,1<<\beta^{-1}<<v
$$

showing that the heated lattice causes the electrons to absorb even more light. Note that the "Q" of the electron's oscillation, $v^{2} /\left(2 \mathrm{Im} x_{v}\right)$, is of order 7 for our estimate eq (11), showing that indeed the optical frequency is much larger than the effective electron collision frequency as we as umed.

One can see at this point that absorption by conduction electrons may cause damage, for, if the electron density were $10^{16} \mathrm{~cm}^{-3}$ at an optical intensity of $1010 \mathrm{~W} / \mathrm{cm}^{2}$ (which the evidence outlined in Section 1 suggests as a conservative estimate), and if eq (11) were valid, then $\omega_{\mathrm{p}}^{2} \sim 10^{-3}$ and eq (8) gives $x_{n} \sim 0.012 \mathrm{~cm}^{-1}$. For a $30 \mathrm{nsc}$ pulse this absorption would deposit $40 \mathrm{~J}$ per cc in the lattice, somewhat less than the value Uo expected to cause damage. However, Belikova, et al. [7], gave convincing evidence that the electron density is increasing very rapidly with optical intensity at these levels, an occurrence which we expect for reasons given in the following section. Before considering factors which affect the electron density, however, we consider contributions other than those of the linear response eq (9) to electron damping.

First, the question arises as to whether there are significant intensity-dependent corrections to the electron response at the optical frequency as represented by eq (9). (Responses at harmonic frequency multiples do not contribute to the average work done by the optical field on the electrons and so we need not consider these here.) There are two types of nonlinear corrections to eq (9): first, those arising from the nonlinear reactions of the phonons on the electron, and, second, those arising from the intensity-dependent deviations of the momentum distribution of electrons from a thermal distribution. We have estimated both effects by expanding the quantum expression for the expected electron position (and velocity) to the third order in the electric field. We have found that both effects tend to diminish the optical absorption from its linear response value, a result expected on the physical grounds that electrons of high energy interact less with the optical phonons than do low-energy electrons. For both corrections the natural dimensionless expansion parameter is $\left(k_{0} r_{0}\right)^{2} w_{h} x_{e} k_{0}$ is the wavevector of an electron having energy $\hbar v$ and $r_{0}$ is the classical amplitude of a free electron oscillating in the applied optical field. In our case this parameter is $\sim \mathrm{I}\left(\mathrm{W} / \mathrm{cm}^{2}\right) \mathrm{x} 10^{-1} \mathrm{C}^{3} \mathrm{Since}$ optical intensities have not yet been observed to approach even $10^{11} \mathrm{~W} / \mathrm{cm}^{2} \mathrm{before} \mathrm{damage,} \mathrm{we} \mathrm{con-}$ clude that the nonlinear corrections to eq (9) are too small to be of importance in present considerations.

Next, we see why, under conditions of interest here, phonon scattering of conduction electrons dominates scattering by ionized and un-ionized lattice impurities. For each conduction electron there is in the lattice a heavy positive ion whose charge is shielded roughly by the static dielectric constant of the lattice $\left(\epsilon_{0} \sim 10\right)$. The linear absorption by 1016 electrons per cc in such a two-component plasma at room temperature is $\sim 1^{-6}$ per cm [11]. This is so small compared with phonon effects that one can readily appreciate that even if $10^{19}$ per $\mathrm{cm}^{3}$ of electrically neutral scattering centers were added to the lattice, one would not expect them to scatter electrons as effectively as do phonons at room temperature and above. This predominance of phonon scattering at or above room temperature has been observed in various ways even in crystals whose electron-phonon coupling is much smaller than in ruby and sapphire [12]. Having estimated the optical response of a single conduction electron, we now proceed to consider how their numbers are affected by their interactions with lattice impurities.

\section{Conduction Electron Interactions with Impurities}

From incomplete existing absorption [13] and luminescence [8] data, it is evident that sapphire has a complex impurity level structure with energy differences ranging up to at least 3 eV. Light of any single optical wavelength, such as $6943 \AA$, will not be resonant with a significant fraction of the bound-bound transitions and, of course, will only excite transitions to the conduction band from bound levels within $1.8 \mathrm{eV}$ of the band edge. If, however, the light became effective in exciting a large fraction of the bound-bound transitions, then cascade transitions of electrons into the conduction band from levels lying more than $1.8 \mathrm{eV}$ below the band edge might dominate direct photo-excitation. We shall now show that, at conduction electron densities $1016 \mathrm{~cm}^{-3}$, the light does become so effective in exciting bound-bound transitions with which it is not resonant that cascade photo-excitation may become important in increasing the conduction electron density near optical damage intensities. These nonresonant transitions are possible because the conduction electrons readily absorb the difference in energy $v$ - $\omega_{b}$ a between an absorbed photon and the energy of transition between the upper and lower states $|b\rangle$ and $|a\rangle$. We now show that the rate $R_{a b}$ for a typical impurity to make such a transition, multiplied by a reasonable impurity density $\left(\sim 1019 \mathrm{~cm}^{-3}\right)$, may exceed the total direct photoexcitation rate, and thereby initiate cascade ionization of impurities.

To calculate the rates $R_{a b}$ it is convenient to re-express the interaction of eq (3) as a Fourier transform (the impurity index $a$ is now omitted since we are considering only one impurity): 


$$
\mathrm{v}_{\mathrm{t}}=\sum_{\mathrm{k}} \mathrm{v}_{\mathrm{i}} \mathrm{e} \stackrel{i \mathrm{i}}{\sim} \underset{\sim}{\sim}
$$

where $v_{k}$ is the impurity operator $4 \pi e^{2} V^{-1} \epsilon_{s}^{-1} k^{-2} \Sigma_{j}$ expik ${ }^{r} j$ and $V$ is a fiducial interaction volume. The sum is confined to the bound impurity electrons as only electronic transitions will be considered. For the transitions of interest we may take $\epsilon_{\mathrm{S}}$ to be the optical dielectric constant $\epsilon$. With the form eq (13) it is a straightforward matter to re-derive the quantum "Golden Rule" for calculating transition rates but with an unperturbed Hamiltonian $h_{0}=\frac{1}{2}\left(p-A_{0} \cos v t\right)^{2}$ instead of $\frac{1}{2} p^{2}$. To lowest order in the electron-impurity interaction, the rate for an electron to scatter from momentum state $|\underset{\sim}{p}\rangle$ to $|\underline{\sim}+\underset{\sim}{\sim}\rangle$ while the impurity goes from $\mid$ a) to $\mid$ b $\rangle$ is

$$
\left.\Gamma(\underset{\sim}{p} \rightarrow \underset{\sim}{p}+\underset{\sim}{k} ; a \rightarrow b)=2 \pi\left|\left\langle b\left|{\underset{\sim}{k}}_{k}\right| a\right\rangle\right|^{2} \sum_{n=-\infty}^{\infty} \delta\left(k^{2} / 2+\underset{\sim}{p} \cdot \underset{\sim}{k}+\omega_{b a}+n v\right) \cdot\left[J_{n} \underset{\sim}{(k} \underset{\sim 0}{r}\right)\right]^{2}
$$

where we have retained the terms for $n$-photon emission and absorption for future comparison with the $\mathrm{n}=1$ term that we are now considering. In the polaron units used here the classical electron oscillation amplitude $r_{0}$ equals E/ $v^{2}$, where the real amplitude $E$ of the optical electric field equals the amplitude in esu divided by $E_{0}=\left(\omega_{0}^{2} / e\right)\left(\hbar \mathrm{mb} / \omega_{0}\right)^{\frac{1}{2}}$. For our parameters E equals (the field in $\left.\mathrm{V} / \mathrm{cm}\right) \div$ $(1.24 \times 106)$. The Bessel function coefficients $J_{n}$ in eq (14) are the exact amplitudes for absorbing (or emitting) $\mathrm{n}$ optical photons in the process. Their arguments are of order $10^{-13} \mathrm{I}\left(\mathrm{W} / \mathrm{cm}^{2}\right)$ and $\mathrm{so}$ the approximation $J_{n}(x) \sim(x / 2)^{n / n}$ ! is accurate here. Consider now the rate $r_{a b}$ for the impurity interacting with one electron in the optical field to make a transition from a state $|a\rangle$ to a state $|b\rangle$, for which $\omega_{\mathrm{ba}}<v$. In terms of the rate eq (14)

$$
\mathrm{r}_{\mathrm{ab}}=\sum_{\mathrm{p}, \mathrm{i}} \mathrm{f}(\mathrm{p}) \Gamma(\mathrm{p} \rightarrow \underset{\sim}{\mathrm{p}}+\underset{\sim}{\mathrm{k}} ; \mathrm{a} \rightarrow \mathrm{b})
$$

where $\mathrm{f}(\mathrm{p})$ is the initial conduction electron distribution and only the $\mathrm{n}=\mathrm{l}$ term in $\Gamma$ is $\mathrm{needed}$. To evaluate $\mathrm{r}_{\mathrm{ab}}$ it remains to estimate the dependence of the matrix elements of $\mathrm{v}_{\mathrm{k}}$ on $\mathrm{k}$. We shall use the "dipole approximation" in which $\left\langle b\left|\operatorname{expik} \cdot \underline{r}_{j}\right| a\right\rangle \sim i k \cdot\left\langle b\left|r_{j}\right| a\right\rangle$. For definiteness we will assume that

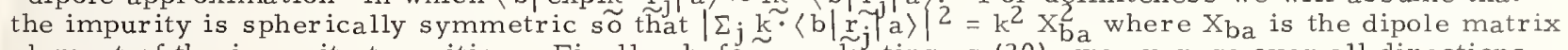
element of the impurity transition. Finally, before evaluating eq (20), we average over all directions of $\mathrm{E}_{\mathrm{N}}$ s that the $\mathrm{J}_{1} 2$ terms becomes $(\mathrm{kE} / 2 \mathrm{v})^{2} / 3$. The $\mathrm{n}=1$ contribution to the sum over $\mathrm{k}$ in eq $(15)$ is thern

$$
r_{a b}=V^{-1} 16 \pi e^{4}\left(r_{0}^{2} / 12\right) e^{-2} x_{a b}^{2} \sum_{p} L(p) f(p)
$$

where

$$
L(p)=\int_{K_{m}-p}^{K} m^{+p} d k k^{2 n-1} / p .
$$

Generally $K_{m} \equiv\left[p^{2}+2\left(v-\omega_{b a}\right)\right]^{\frac{1}{2}}$ is much larger than the magnitude of $\mathrm{p}$ allowed in $\mathrm{f}(\mathrm{p})$. In this case, it is sufficiently accurate to approximate eq (16b) by

$$
L \sim 2\left[2\left(v-\omega_{b a}\right)\right]^{\frac{1}{2}}
$$

and we see that the impurity excitation rate is essentially independent of the initial small conduction electron momentum $\mathrm{p}$. Since $\Sigma_{\mathrm{p}} \mathrm{f}(\mathrm{p})=1$, this fact makes the sum in eq (16a) trivial. The total rate $R_{a b}$ for an impurity to absorb a photon is $x_{a b}$ times the number of conduction electrons in the interaction volume $V$. It is convenient to express $R_{a b}$ in terms of a photon absorption cross-section $\sigma a b$ which equals $R_{a b} \div$ (the incident photon flux). Recalling that $2 v \mathrm{r}_{0}^{2}$ is numerically $10^{-13} \mathrm{I}(\mathrm{W} / \mathrm{cm} 2)$, we have from eq (15) for sapphire

$$
\sigma_{\mathrm{ab}} \sim 7.5 \times 10^{-11} \mathrm{I} \mathrm{X}_{\mathrm{ab}}^{2} \epsilon^{-2} v^{-1}\left(v-\omega_{\mathrm{ba}}\right)^{\frac{1}{2}} \rho
$$

in polaron units where the electron density 0 e is $6.15 \times 10^{-22}$ times the number of electrons per cc 0 . 
To see the importance of the transition cross-section eq (18) relative to direct photo-excitation, we estimate the density of impurities $\mathrm{N}_{\mathrm{i}} \mathrm{cm}^{-3}$ that would be required to attenuate the optical beam more than does the direct photo-excitation process. Of course, the latter attenuation is not known, but it must be less than the total attenuation in sapphire, and that is less than $10^{-2}$ per $\mathrm{cm}$. For argument, suppose that direct photo-excitation causes less than $10^{-3}$ per $\mathrm{cm}$ optical attenuation (it probably causes much less). For our estimate, let us assume in eq (18) that $\mathrm{X}_{\mathrm{ab}}^{2} \sim 1 / \omega_{\mathrm{ab}}, \omega_{\mathrm{ab}} \sim 8, \mathrm{~g}$, a $\mathrm{I}=1010 \mathrm{~W} / \mathrm{cm}{ }^{2}$ and $\rho=1016$ as in previous examples. This gives $\sigma_{a b} \sim 10^{2} 2 \mathrm{~cm}^{2}$ so that $N_{i} \sim 1019$ impurities per cc would be required to make these conduction-electron-mediated impurity transitions attenuate the optical beam by $10^{-3}$ per $\mathrm{cm}$. Considering all the kinds of dislocations and foreign ions which might take part as impurities in this process, we feel that it is not unreasonable that such an impurity density could exist. Of course, there are so many order-of-magnitude estimates of unknown quantities in the foregoing that the result can only be considered as suggestive of a kind of process that must be considered as competitive with other possibilities at this time. However, the experimental observation of a rapidly increasing dc photoconductivity near damage thresholds [7] does suggest a "bootstrap" process such as the above by which conduction electrons can create more of their kind, even before they become so energetic as to ionize the lattice.

We have examined the enhancement of the single-photon bound-free photo-excitation transitions that arise from eq (14), and have estimated that excitation cross-section also to be of order $10-22$ cm 2 under the same conditions. This is probably less than the cross-section for direct photo-excitation. If multi-photon excitation is important in producing electrons, then the higher $\mathrm{n}$ terms of eq (14) probably dominate direct absorption near damage levels. The expansion parameter in eq (14) is of order $10^{-13} \mathrm{I}\left(\mathrm{W} / \mathrm{cm}^{2}\right)$, whereas it is $\sim 10^{-17} \mathrm{I}$ for direct multi-photon absorption.

\section{Focusing Effects}

In a region of the crystal in which conduction electrons have been excited there is a change in the refractive index on given by

$$
\delta_{n}=-\omega_{p}^{2} / v^{2} .
$$

At a density $10^{16} \mathrm{~cm}^{-3}$, electrons (with free electron band mass) have a plasma frequency of $10^{12} \mathrm{~Hz}$, which gives $\omega_{\mathrm{p}} / v \sim 0.0023$ and $\delta_{\mathrm{n}} \sim 5.3 \times 10^{-6}$. This index change is comparable to that required to self-focus a one milliradian gaussian beam. If the electron density were proportional to the optical intensity $I$, and this index change were produced when $I=1010 \mathrm{~W} / \mathrm{cm}^{2}$, then the index change would constitute a second order nonlinear index (often written $\mathrm{n}_{2} \overline{E^{2}}$ ) whose nonlinear coefficient n2 would be $-2 \times 10^{-13}$ esu. This is negative and an order of magnitude larger than what one would guess is the intrinsic nonlinear index of the bound electrons in sapphire. However, we suspect that the electron density is increasing much more rapidly than I at high intensities [7] and so the effect of the index of eq (19) is not simple. Furthermore, impurity electrons which are excited to bound states more than $v$ below the band edge, such as by the process discussed in the previous section, have an increased polarizability and would tend to cancel the effect eq (19) of conduction electrons.

Suppose the net effect of impurity transitions were to produce a negative and highly nonlinear refractive index change, as would be the case if the conduction electron effect eq (19) dominated. Then there would be strong self-defocusing of a beam in regions of very high intensity, but only the intrinsic self-focusing index would be operative in regions of smaller intensity. This would evidently result in successive regions of high intensity along the beam as each effect produces in turn a focusing tendency that brings the other effect into dominance. Damage tracks in sapphire and other materials consist of a sequence of damage bubbles that are suggestive of such a process. However, we have not calculated the spacing of the successive high-intensity regions on the basis of some reasonable model to see whether there is any possible correspondence between these speculations and observation.

\section{Discussion}

Although there are alternate explanations for the behavior of damage tracks observed in sapphire [14], it seems likely that self-focusing is occurring and playing a role in the damage. (It is curious that the appearance, lengths, and positions of damage tracks differ between ruby and sapphire [14].) If self-focusing is occurring, it makes especially difficult the determination of the actual optical intensities at which the material ruptures, and this in turn lends an extra uncertainty to the parameters we have used in discussing the role of conduction electrons in damage. Probably the most promising way to determine more precisely what the electrons' role may be is to determine the parameters of these conduction electrons below damage intensities. It would be especially desirable to have reliable dc photoconductivity measurements between the intensities $5 \mathrm{~W} / \mathrm{cm}^{2}$, the highest used by Hochuli [5], and $1010 \mathrm{~W} / \mathrm{cm}^{2}$, the lowest used by Belikova, et al. [7], in their measurements. With the high light intensities available with lasers, it may now be possible to excite enough electrons to obtain a 
meaningful Hall effect. Perhaps the time decay of photoconductivity and luminescence after an exciting pulse could be observed and yield information on carrier lifetimes, trapping times, etc. Unfortunately, the important and pervading parameter - the effective mass in the conduction band (and its anisotropy) appears to be extremely difficult to determine experimentally.

Should the photo-excited electrons be found to play significant roles in damage, such as those we have discussed, then it may be possible to fabricate more damage-resistant sapphire and ruby. For example, if self-focusing is presently instrumental in damage, then it might be reduced by adding donor impurities that produce a linear photoconductivity at high intensities and thus reduce the total nonlinear index via the index change of eq (19). On the other hand, those donors that give a highly nonlinear photoconductivity and a sudden production of high electron densities that result in rupture by photo-absorption may perhaps be eliminated from the crystals or compensated by other additives. In any case, it is evident that the availability of high-intensity laser sources will greatly facilitate the study of the conduction bands and impurity levels in very weakly photoconducting materials such as sapphire and ruby.

\section{References}

[1] A. Wasserman, "A mechanism for damage in solids by intense light," Appl. Phys. Letters 10, $132-133$ (1967).

[2] V. A. Pashkov and G. M. Zverev, "Destruction of ruby and leucosapphire crystals by strong laser radiation, " Soviet Phys. -JETP 24, 516 518 (1967).

[3] E. A. Sviridenkov, "Mechanism of damage of ruby by laser radiation, "Soviet Phys. -Solid State 9, 1917-1918 (1968).

[4] G. M. Sverev, T. N. Mikhailova, V. A. Pashkov, and N. M. Solov' eva, "Mechanisms of destruction of ruby and leucosapphire crystals by powerful laser radiation, " Soviet Phys. - JETP 26, 1053-1057 (1968).

[5] Urs E. Hochuli, "Photoconductivity in ruby and sapphire," Phys. Rev. 133A, 468-471 (1964).

[6] R. P. Feynman, R. W. Hellwarth, C. K. Iddings, and P. M. Platzman, "Mobility of slow electrons in a polar crystal," Phys. Rev. 127, 1004-1017 (1962). Hereinafter referred to as FHIP.

[7] T. P. Belikova and E. A. Sviridenkov, "Photoconductivity of ruby when strongly irradiated by a ruby laser," JETP Letters 3, 257-259 (1966).

[8] T. P. Belikova, A. N. Savchencko, and E. A. Sviridenkov, "Optic breakdown in ruby and related effects," Soviet Phys. - JETP 27, 19. 23 (1968).
[9] L. Spitzer, Jr., Physics of Fully Ionized Gases (Interscience Publishers, New York, 1956).

[10] H. Frohlich, "Theory of electrical breakdown in ionic crystals," Proc. Phys. Soc. (London) Al60, 230-241 (1937).

[11] V. I. Perel and G. M. Eliashberg, "Absorption of electromagnetic waves in plasma," Soviet Phys. -JETP 14, 633-637 (1.962).

[12] R. H. Bube, Photoconductivity of Solids (John Wiley and Sons, New York, 1960).

[1 3$]$ N. V. Beardsley, Proceedings of the Infrared Information Symposia, Volume 1, No. 2, 47$53(1956)$.

[14]C. R. Giuliano and L. D. Hess, "Laser-induced damage in ruby and sapphire," in these Proceedings.

\section{COMMENTS ON PAPER BY ROBERT HELLWARTH}

The model employed in this paper was specifically developed for crystalline solids containing impurities. This model, involving definite impurity levels imposed on a well-defined band structure, does not apply to glasses in its present form. 
Damage Threshold Studies in Ruby and Sapphire ${ }^{1}$

\author{
C.R. Giuliano and L.D. Hess \\ Hughes Research Laboratories \\ Malibu, California 90265
}

\begin{abstract}
In this presentation a mode-controlled giant pulse ruby laser oscillator and amplifier are described. The oscillator employs a temperature-controlled resonant reflector, a dye Qswitch, and a $2 \mathrm{~mm}$ aperture to assure single mode operation. The results of damage threshold measurements on a few ruby and sapphire samples are discussed. Scanning electron micrographs of surface damage, and photomicrographs of bulk and surface damage are presented. The dependence of damage threshold on $\mathrm{TiO}_{2}$ doping and optical pumping is discussed, and some marked differences in the damage phenomenon between ruby and sapphire are pointed out.
\end{abstract}

Key Words: Bulk damage, damage thresholds, lasers, mode control, optical pumping, ruby, sapphire, surface damage.

\title{
1. Introduction
}

In this presentation we wish to describe some experimental work that has been carried out as part of a continuing program to study laser induced damage in solids. We will first describe the apparatus used in the damage experiments. We will then discuss some of the gross physical characteristics of both surface and bulk damage. We will present data on damage thresholds for a number of different samples, and discuss the effects on the damage threshold of adding titanium dioxide to ruby and sapphire.

Finally, we will present the results of experiments in which we optically pump the samples while irradiating them with the laser.

Since the earliest observations of laser-induced surface [1] ${ }^{2}$ and bulk [2] damage, there has been a large volume of published work [3-10] covering many aspects of this phenomenon in a variety of solid materials. Many mechanisms have been proposed and discussed, and considerable threshold data have been reported; despite this intensive effort, a number of questions remain to be answered. Our approach is to use a laser whose output properties are well characterized and monitored, so that any fluctuations in the data must be attributed to something other than fluctuations in the source.

\section{Experimental Apparatus}

The experimental setup is shown in Fig. 1. The oscillator employs a 4 in. long $\mathrm{x}$ 1/4 in. diameter ruby pumped by two linear lamps in a double elliptical pump cavity. The ruby crystal is water-cooled by a closed-cycle refrigeration system maintained at $0^{\circ} \mathrm{C}$. The high reflectivity mirror is coated with a $99+\%$ reflectivity high field damage coating from Perkin Elmer Corporation. Q-switching is accomplished with a solution of cryptocyanine in methanol in a $1 \mathrm{~mm}$ path length cell whose transmission is $30 \%$ at 6943 \&.

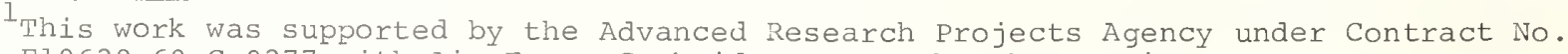
F19628-69-C-0277 with Air Force Cambridge Research Laboratories.

2 Figures in brackets indicate the literature references at the end of this paper.
} 
The temperature controlled $\left(34^{\circ} \mathrm{C}\right)$ resonant reflector that was designed to optimize longitudinal mode control consists of two quartz etalons and a quartz spacer whose combined effect is to enhance cavity modes separated by $2 \mathrm{~cm}^{-1}$ and to discriminate against intermediate modes.

Portions of the laser beam are split off in various ways (see Fig. 1), so that the power output, near- and far-field patterns, and Fabry-Perot patterns can be monitored for each shot. This is accomplished in the following way. Light reflecting from wedged beamsplitter $W_{l}$ gives two diverging beams; one of them hits the magnesium oxide diffuser, where the scattered light is monitored by a biplanar photodiode used as our power monitor. The second beam from $W_{1}$ hits ground glass screen $G$, where it is photographed through lens $L$ and the $1 \mathrm{~m}$ focal length camera focused at infinity. This gives a magnified $(\sim 5 x)$ near-field picture. Another portion of the light is removed by beamsplitter $B_{1}$ and hits mirror $M_{2}$, which can be placed in or out of position depending on the use of the alignment laser. From $\mathrm{M}_{2}$ the light either goes to the Fabry-Perot interferometer or can be partially reflected from wedged beamsplitter $w_{2}$, where it results in a pair of far-field patterns. A 0.6 neutral density filter is placed near the focal plane of the camera so that the far-field pattern and the Fabry-Perot pattern can be seen at two different exposures. The two Glan prisms are used as a variable attenuator after the amplifier. Beamsplitter $\mathrm{B}_{2}$ samples the light to photodiode No. 2, which monitors the power incident upon the focusing lens, which was designed for minimum spherical aberration (Special optics). Photodiode No. 3 monitors the light after the sample. The signals from the two detectors are integrated and displayed on a dual beam oscilloscope.

The water cooled amplifier ruby is $6 \mathrm{in.}$ long $\mathrm{x} 0.5 \mathrm{in}$. diameter, with one end wedged relative to the other by about $0.5^{\circ}$. The input end of the amplifier rod is antireflection coated to minimize the chances of oscillation within the amplifier itself. The ruby rod is closely coupled to a helical flashlamp, which is pumped with a power supply capable of delivering $8 \mathrm{~kJ}$ in a $3 \mathrm{msec}$ pulse. The power supply employs a pulse shaping network of 20 sections, each section pumping for 150 usec. The maximum gain obtained with the amplifier is about $10 \mathrm{~dB}$.

Figure 2 shows an example of what we see with our monitoring camera, and Fig. 3 shows a time trace of the laser output taken with photodiode No. 1 and displayed on the Tektronix 519 oscilloscope. Figure 3(a) shows the smooth temporal shape seen about 95\% of the time, while Fig. $3(\mathrm{~b})$ is an extreme example of multimode oscillation. In this case two modes are oscillating with a frequency separation of $750 \mathrm{MHz}$. This frequency corresponds to the spacing between the end of the ruby and the resonant reflector. The overlapping spectral ranges of the Fabry-Perot interferometer and the 519 oscilloscope insure that the oscillation of anything other than a single longitudinal mode will be detected.

The characteristics of the laser are summarized in Table 1.

Table 1. Characteristics of ruby laser.

\begin{tabular}{|l|l|}
\hline $\begin{array}{l}\text { Energy Output } \\
\text { Pulse Length }\end{array}$ & $12-15 \mathrm{~mJ}$ ( $\pm 15 \%)$ \\
$\begin{array}{l}\text { Peak Power } \\
\text { Beam Radius (1/e for E } \\
\text { field) }\end{array}$ & $0.6-0.8 \mathrm{MW}$ \\
Beam Divergence & $0.6 \pm 0.1 \mathrm{~mm}$ ( $\pm 10 \%)$ \\
Calculated Beam Divergence (full angle) & $0.44 \mathrm{mrad} \mathrm{(full} \mathrm{angle)}$ \\
\hline $\begin{array}{l}\text { aur energy monitor was checked against three different } \\
\text { "calibrated" detectors; the error quoted ( } \pm 15 \% \text { reflects } \\
\text { the disagreement of the standards. }\end{array}$ \\
\hline
\end{tabular}


We now wish to point out some of the qualitative features of the damage we have observed in ruby and sapphire, and point out the various differences seen in bulk and surface damage.

When we place these samples in the beam we often see some plasma formation at the entrance and exit surfaces; this is shown in Fig. 4(a). In this shot no internal damage was formed, but a small pit was visible at the exit surface. We wish to emphasize here that exit surface damage is the first to appear in most of our measurements and that the threshold for exit surface damage is about an order of magnitude lower than that for bulk damage. We also notice that the plasma formed at the exit surface is often irregular, sometimes with a pointed appearance in the direction of light propagation; the plasma formed at the entrance surface is more rounded in appearance. Figure $4(\mathrm{~b})$ shows a photograph of another sample in which internal as well as exit surface damage was formed. Here we see a damage track beginning about a third of the way in from the entrance surface. Notice also the flaring out of the beam past the damage track. This blowing up of the beam could be caused by a scattering of light from the damage sites or perhaps by self-focusing and subsequent diffraction. If we assume that the fanning out of the beam is caused by diffraction from a self-focused spot, we can determine roughly the spot size by measuring the angle of the fanned out part of the beam. A crude measurement gives $7 \times 10^{-2}$ rad for the half-angle, which corresponds to a self-focused spot whose diameter is $6.3 \mu \mathrm{m}$. This is not an unrealistically small spot size for self-focusing, and thus the flared out beam could arise from that. On the other hand, we notice that the flared out part of the beam is not very uniform in that the center part has much more light than the outer portions. This would suggest scattering from the damage sites in which a small part of the beam is deflected while most of it continues along the same path.

\subsection{Surface Damage}

The difference in the qualitative features of entrance and exit surfaces will be discussed briefly in this section. Figure 5 shows photographs of both the entrance and exit faces of a ruby rod subjected to varying amounts of laser radiation. Note the difference in the kind of damage seen. The exit surface generally shows definite crater formation, while a relatively small amount of material is lost from the entrance surface.

The influence of entrance surface damage on the apparent threshold for bulk damage has come to our attention recently in this work. It is possible that one reason for the large fluctuation in the bulk damage threshold is the generation of damage at the entrance surface and subsequent scattering of light from this damage site (or absorption by the plasma), which could result in a much lower power density in the interior of the sample than expected. It has been observed that once this entrance surface damage has been formed it is very difficult, if not impossible, to create bulk damage with subsequent shots in the same place. For example, when the same region of the sample is repeatedly irradiated with the laser, each time with increasing power, the bulk damage threshold often cannot be reached; however, the damage on both surfaces generally increases from shot to shot. The problem of surface damage varies considerably from sample to sample and from place to place in a given sample. No substantial difference in this behavior is seen when the surfaces are cleaned with hot nitric acid and rinsed thoroughly with deionized water. (Generally all surfaces are cleaned with ethyl alcohol.)

The photographs in Fig. 5 illustrate that there is a spatial correspondence between the entrance and exit surfaces. As an example of the variation in results for different parts of the sample, let us compare the different regions marked on the photographs. In region $\mathrm{A}$, for example, both entrance and exit damage are observed in a location subjected to several shots from the laser. This region was not damaged internally, even though the power incident on the sample far exceeded that for which internal damage was created in other regions (e.g., region $B$ ). Region $B$ is one in which both internal damage and exit surface damage were generated (in this case with one shot), with barely noticeable entrance surface damage. Region C shows a location subjected to a single shot of less than half the totai energy as in B. In this case no internal damage was formed but the exit crater is considerably larger than that seen in B. Figure 6 shows entrance and exit surfaces of another sample, where we again see the qualitative features of the different kinds of damage formed. Note the general anticorrelation between the extent of entrance and exit damage as exemplified in Regions $A$ and $B$. In one case (A) we see exit damage with little or no entrance damage, and in the other we see just the opposite. 
Next let us compare the entrance and exit surface damage at somewhat higher magnification. Figure 7 shows a magnified view of one of the damage sites seen on the entrance surface of the sample shown in Fig. 6. The feature we wish to point out here is the crazed appearance of the surface. There appears to be a general direction to the crazing on the surface that is the same for all the damage sites examined on that crystal. Other crystals also show this type of linear crazing, but it is not seen on all the samples examined. Figure 8 shows magnified views of exit surface damage.

Note that the exit surface damage consists of pitting or crater formation, as well as the crazing similar to that seen on the entrance surface.

We have used a scanning electron microscope to examine the damage pits formed at the exit surface of a ruby sample. The particular sample is a $1 / 4$ in. diameter $x$ in. long rod. Figure 9 shows several views of the surface damage under different magnifications. The first photograph (Fig. 9(a)) shows the rod under 23x magnification. The damaged areas were caused by three different shots of the laser; the one small pit at the lower left was caused by one shot, the cluster at the lower right by another, and the large craters at the top by a third. Further magnification of the last two regions is seen in the other electron micrographs in the figure. Figures 9 (c) and (d) show the large crater at the top magnified $610 x$ and $7000 x$, respectively. Figures 9 (e) and (f) show one of the multiple pits on the lower right magnified 660x and 6600x, respectively. Electron micrographs of the undamaged surface at $7000 x$ magnification show essentially a smooth surface (Fig. 9(b)). The magnified view of the large crater in Figs. 9 (c) and (d) shows what appears to be a molten region around the hole, while the views of the pitted area (Figs. 9 (e) and (f)) appear more as multiple fractures than a melting phenomenon.

\subsection{Bulk Damage}

During these studies we have noticed a difference between the qualitative features of the bulk damage in ruby, compared with sapphire. This is illustrated in Figs. 10 and 11. In Fig. 10 we see some typical damage tracks seen in a ruby sample. These tracks appear to be a series of bubbles or small fractured regions of variable spacing; they are more densely packed at the beginning of the track and spread out toward the end, so that in some cases there is an appreciable gap between damage sites in a given damage track. The beginning of the track generally shows a more densely damaged region that is usually much wider than the rest of the track. In contrast, the bulk damage in sapphire appears to be qualitatively different. In Fig. 11 we see an example of typical damage tracks observed in sapphire. These are generally more continuous (fewer interruptions) than those seen in ruby, and often contain a "hollow" core with fractured regions at the periphery (see Fig. $11(\mathrm{a})$ ).

In this particular sample there were a few irregular tracks such as that shown in Fig. $11(\mathrm{~b})$. It is curious to note that these tracks occurred when there was appreciable temporal modulation on the laser pulse. When these measurements were made there was an unusually high percentage of modulated shots. It was noted that the irregular damage occurred only on those shots when the pulse was modulated (about six times). We offer no interpretation to this observation, but merely point it out as a curious occurrence.

This section may be summarized with the following statements concerning the qualitative features of the damage in ruby and sapphire.

There are three distinctly different types of damage: bulk, entrance surface, and exit surface damage. The surface damage appears to be similar in both ruby and sapphire, while the bulk damage differs as described above.

Entrance and exit surface damage are similar in two respects and differ in a third. They are similar in that plasmas occur at both surfaces and both show some crazing at the damage site. They differ in that a fracture crater occurs on the exit and not on the entrance surface. We suspect that the crazing is connected with and perhaps caused by the plasma that is seen at both surfaces, whereas the crater, which is unique to the exit surface, is generated by a phenomenon that takes place in the bulk of the material and manifests itself at the surface.

The threshold for exit surface damage is about an order of magnitude lower than that for bulk damage. It is more difficult to make a definitive statement concerning the relative thresholds for entrance surface damage and internal damage. The ease of plasma formation at the entrance appears to be a function of undetermined surface 
parameters, the most likely of which are adsorbed substances and surface finish. ${ }^{3}$ Therefore, the state of the entrance surface definitely determines what occurs inside the crystal and at the exit surface. In the extreme case all of the light could be dissipated in the entrance plasma.

Finally, let us assume that a hypothetical sample is subjected to a series of shots with increasing energy, each time striking a different spot, and assume that the power density is uniform over the length of the sample. We will first observe exit surface damage at some threshold. As the energy is increased, the extent of the exit surface damage will increase to give a larger crater than on the previous shot. At an order of magnitude higher input we reach the threshold for bulk damage, at which point a short damage track will appear; at the same time, we will probably observe a decrease in the size of the exit pit relative to what it was just below bulk damage threshold. As we increase the energy further we will generally observe more extensive internal damage and perhaps begin to see some entrance surface damage, depending on the condition of the entrance surface. At still higher incident energy the extent of entrance surface damage (or absorption by the plasma) can be so great that it precludes the formation of bulk damage.

\section{Damage Threshold Experiments}

In this section we present the results of damage threshold experiments for internal damage in a number of different materials from various sources. In all the cases presented the data represent a large number of shots for a given sample. There was a large amount of scatter in the data, as mentioned earlier; we believe this results in part from the varying amount of plasma formed at the entrance surface. It is also reasonable to believe that there is an intrinsic variation in damage threshold from place to place in the same sample. As a result, there is a range of incident energies over which one may or may not see internal damage, depending on the particular location in the crystal. For example, at a particular location in the sample no damage is seen at a given incident energy, while extensive damage may be seen in another part of the sample for a lower energy in another shot. The data presented in Fig. 12 reflect this fact. The dashed line corresponds to a range of power densities where damage was not observed at some locations in a given sample and is terminated on the high end by the highest power for which internal damage was not created. The solid line corresponds to input energies where damage was observed at some locations in the same sample and is terminated on the low end by the smallest input energy for which damage was observed. For example, let us examine the data presented for Cz Ruby L104 in Fig. 12. The dashed line shows that certain regions of the sample were subjected to power densities up to $7.1 \mathrm{GW} / \mathrm{cm}^{2}$ without damage. The solid line shows that damage was observed in some other parts of the sample at input power densities as low as $6.3 \mathrm{GW} / \mathrm{cm}^{2}$. Thus, the amount of overlap gives a measure of the definability of damage threshold for a given sample.

The power densities in Fig. 12 are calculated power densities at the beam waist. Mode propagation equations described previously [11] were used to compute beam radius and divergence at various locations. The energy incident on the focusing lens first is measured by photodiode No. 2 (Fig. 1). This is converted to power by dividing by the appropriate pulse width (FWHM) as determined by the Tektronix 519 oscillograms. The power density at the lens is computed by dividing this power by the area of the beam at the lens. This gives a spatial average for the power density or energy density. The energy density at the peak of the gaussian distribution is twice the spatial average. The power density at the focus of the lens is obtained by computing the beam size at the focus.

\footnotetext{
It is definitely known that a substantial plasma will form on a dirty entrance surface. In addition, in our limited observations it appears that those crystals with more surface scratches are more easily damaged at the entrance surface.
}

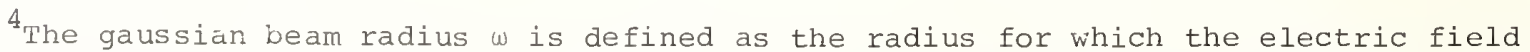
reaches $1 /$ e of its peak value. This is the radius for which the intensity or the energy density reaches $1 / \mathrm{e}^{2}$ of its peak value. If we define a beam area $\mathrm{A}=\pi \omega^{2}$

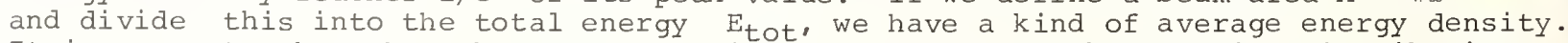
It is easy to show that the energy density at the peak of the gaussian distribution is $2 \mathrm{E}_{\text {tot }} / \pi \omega^{2}$. In most of the reports in the literature the power densities or energy densities refer to a kind of average. That is, the total energy is divided by the "beam size" determined by some means or other. This method is convenient when the spatial power distribution is not known. 
We wish to emphasize that these power and energy densities are based on the following assumptions: (1) The spatial profile of the output is gaussian with a $1 \mathrm{~mm}$ radius (1/e radius for the electric field.) (2) The spreading of the beam is by diffraction only, and the beam suffers no distortion, focusing, or defocusing in passing through the ruby amplifier. (3) The diffraction limited spot size is reached at the focal plane of the focusing lens. The degree to which these assumptions reflect the reality of the physical situation can only be estimated at present, pending further measurements. Measurements of the near field spot size at the oscillator output mirror give a $1 \mathrm{~mm}$ radius for the l/e points of the electric field (assuming a gaussian distribution). This was done both by photographing a pair of spots on a ground glass screen with a known attenuation difference and by measuring the sizes of burn patterns on Polaroid film, with the relative energies of successive shots known. The measurements by both techniques agree to better than $15 \%$. Measurements of the farfield divergence of the oscillator give values that are higher than that expected for a gaussian, although the measurements are not highly accurate. We obtain $0.6 \pm 0.1$ mrad (full angle) from relative spot size measurements in the focal plane of the $1 \mathrm{~m}$ focal length camera. The calculated beam divergence gives $0.44 \mathrm{mrad}(\theta \simeq$ $(2 \lambda) /\left(\pi \omega_{0}\right)$; full angle); this is appreciably lower than that observed but is almost within the uncertainty of measurement. Even though we are well below the saturation flux for the amplifier ( $4 \mathrm{~J} / \mathrm{cm}^{2}$ compared with $15 \mathrm{~J} / \mathrm{cm}^{2}$ ), the uncertainty in the effect on the beam properties of the amplifier is probably the largest and most difficult to estimate.

\section{Dependence of Damage Threshold on Added $\mathrm{TiO}_{2}$}

Recent work of Nath and Walda [12] reported a striking increase (40x) in the damage threshold in sapphire when small amounts (20 to $100 \mathrm{ppm})$ of $\mathrm{TiO}_{2}$ were added. We decided to check these results and extend them to ruby as well. We purchased Verneuil ruby and sapphire samples from the same source used by the above workers (Djeva in Switzerland via Adolf Meller Co.), both undoped and doped with nominally $30 \mathrm{ppm} \mathrm{TiO}_{2}$. The threshold for internal damage was measured, and the results were found to be essentially opposite those reported previously. Table 2 shows the results of these measurements. The numbers in the table are given in pairs. As discussed in connection with the data in Fig. 12, the numbers reflect the variation of threshold from place to place in the sample. Of the two numbers quoted, that on the left is the highest value for which damage was not observed in the sample, and that on the right is the lowest value for which damage was found in the same sample. We see from Table 2 that the thresholds for the titanium doped samples are at least an order of magnitude lower 5 than those for undoped samples. The reason for this discrepancy with the results of Nath and Walda is not known. In addition, contrary to the results of Nath and Walda, we found that the ultraviolet absorption edge for the $\mathrm{TiO}_{2}$ doped samples occurred at a shorter wavelength than that for the undoped sapphire (2600 versus $2300 \AA)$.

Table 2. Effect of $\mathrm{TiO}_{2}$ doping on damage threshold.

\begin{tabular}{|l|r|c|}
\cline { 2 - 3 } \multicolumn{1}{c|}{} & \multicolumn{2}{c|}{$\begin{array}{r}\text { Power Density for Internal } \\
\text { Damage, GW/ } / \mathrm{cm}^{2}\end{array}$} \\
\cline { 2 - 3 } & Without $\mathrm{TiO}_{2}$ & With $30 \mathrm{ppm} \mathrm{TiO} 2$ \\
\hline Verneuil Ruby & $5.4,9.9$ & $0.94,0.70$ \\
Verneuil Sapphire & $12.1,9.4$ & $0.36,0.52$ \\
\hline
\end{tabular}

Because of these discrepancies it is reasonable to suspect that we may in fact have different material from that stidied by Nath and Walda. These titanium doped samples are examples of ruby and sapphire for which the bulk
damage threshold is so low that surface damage does not form. 
During this reporting period we have carried out a few experiments in which we optically pumped the samples while subjecting them to the laser radiation. The experiments were carried out on two ruby samples and one sapphire sample.

The samples were pumped with a power supply similar to that used for pumping the amplifier flashlamp. In these experiments we pumped at two different levels (1200 J and $3700 \mathrm{~J}$ ) into the flashlamp (model FX60 by E.G.\&G). As in the previous experiments we fired a number of shots (generally 10 to 20 ) at each pumping level for a given sample.

The results of the threshold versus pumping experiments are shown in Fig. 13. Here the results are depicted in a manner similar to that of Fig. 12, where the dashed line refers to the powers where no damage was seen in some parts of the crystal and the solid line to powers where damage was seen in other parts. The scatter in the data is relatively high, but nevertheless we can confidently say that there is no appreciable lowering of the threshold with optical pumping and, if anything, there may be a slight increase.

One reason for the scatter in this case is the use of a shorter focal length lens than has been generally used (19 cm compared with $48 \mathrm{~cm}$ ). We used this lens because the output from our system was somewhat lower than previously and we were not quite able to reach damage threshold with the long focal length lens. A more extensive plasma was formed at the entrance surface at times when the $19 \mathrm{~cm}$ lens was used, and an appreciable fraction of the light undoubtedly was absorbed by the plasma.

We observed another interesting phenomenon in these pumping experiments related to the location of the damage in the crystal as a function of pumping. In ruby that is not externally pumped, we see damage tracks whose beginning is usually uniquely located in the sample. That is, for a number of shots at different powers we find that the damage tracks begin at roughly the same distance from the entrance surface. The length of a given track generally will be longer for higher power.

When the ruby samples are optically pumped, we see damage tracks that begin at an appreciably different location "downstream" from the tracks for the unpumped sample. Harder pumping shifts the tracks even more. Results for a sequence of nonpumping, pumping, nonpumping, pumping are reproducible, showing that the phenomenon is reversible.

The results of these experiments are shown in Fig. 14, where the location of the beginning of the damage track is plotted as a function of energy discharged into the flash lamp. The lengths of the bars on the plot reflect the variation in position from shot to shot of the beginning of the damage track.

This effect of apparent defocusing of the light cannot be explained as a sort of thermal focusing due to the optical pumping; it is much too large for that. For example, if we were to assume that the ruby has somehow become a negative lens, we might ask what the effective focal length would be. Taking into account the distance of the entrance surface of the ruby from the lens and assuming that the shift in the location of the damage track corresponds to a shift in focus, we compute that the ruby would have to act as a negative lens of $220 \mathrm{~cm}$ focal length, which corresponds to a radius of curvature of the end of the rod of $\sim 7 \mathrm{~cm}$.

Altematively, we can compute the apparent change in refractive index with pumping to account for the shift in the position of the damage track. The relative shift in the distance from the entrance surface to the focus is directly proportional to the relative change in refractive index. This then corresponds to an apparent index change of 40 to $50 \%$, a very large effect!

It is difficult to account for this effect by postulating any change in the linear refractive index. A decrease in nonlinear index that somehow depends on the degree of optical pumping is a possible explanation, but this should result in a higher damage threshold for pumping than for nonpumping. This may be the case, but there is too much scatter in the data to confirm this (Fig. 13). We also mentioned that in the pumping experiments there was a substantial amount of plasma formation at the entrance surface. It is conceivable that optical pumping of the surface plasma could lead to some defocusing effect. It is clear that more experiments in this area are needed, especially with regard to the relative damage thresholds. 
A correlation for sapphire similar to those seen for ruby in Fig. 14 does not exist. The location of the beginning of the damage tracks in unpumped sapphire varies over a much wider range, depending on the incident energy from the laser, and any effect of optical pumping is obscured by this variation.

When the incident laser power is close to threshold the damage is found farther "downstream"; as the incident energy increases, the location of the beginning of the track moves "upstream" toward the source. [8] This is illustrated in Fig. 15, where we plot the distance of the beginning of the damage tracks from the entrance surface versus laser power incident on the focusing lens.

\section{Conclusion}

Qualitative features of surface damage are shown to be quite different for exit and entrance surfaces. The difference between laser-induced internal damage in ruby and sapphire, both in the gross and more subtle aspects, is pointed out both in actual appearance of the damage tracks and in the dependence of the location of the tracks on input laser energy and optical pumping. Perhaps these differences can be attributed solely to the presence of chromium ions in ruby. At any rate, the effect of chromium concentration on these phenomena should be studied. Further studies on these materials are in progress.

\section{References}

[1] Giuliano, C.R., "Laser-induced damage to transparent dielectric materials," Appl. Phys. Letters 5, 137-139 (1964).

[2] Hercher, M., "Laser induced damage in transparent media," Opt. Soc. Am. $54,563(1964)$.

[3] Belikova, T.P. and Sviridenkov, E.A., "Effect of a focused ruby laser beam on ruby," JETP Letters 1, 171-172 (1965).

[4] Ashkinadze, B.M., Vladimirov, V.I., Likhachev, V.A., Ryvkin, S.M., Salmanov, V.M., and Yaroshetskii, I.D., "Breakdown in transparent dielectrics caused by intense laser radiation," Soviet Physics - JETP 23, 788-797 (1966).

[5] Pashkov, V.A. and Zverev, G.M., "Destruction of ruby and leucosapphire crystals by strong laser radiation," Soviet Physics - JETP $24516-518$ (1967).

[6] Belikova, T.P., Savchenko, A.N., and Sviridenkov, E.A., "Optic breakdown in ruby and related effects," Soviet Physics - JETP, 27 19-23 (1968) .
[7 [ Zverev, G.M., Mikhailova, T.N., Pashkov, V.A., and Solov'eva, N.il., "Mechanism of destruction of ruby and sapphire crystals by powerful laser radiation," Soviet Physics JETP 26, 1053-1057 (1968).

[8] Zverev, G.M., Maldutis, E.K., and Pashkov, V.A., "Development of self-focusing filaments in solid dielectrics," JETP Letters 9, 61-63 (1969).

[9] Dupont, H., Donzel, A., and Ernest, J., "On laser-induced breakdown and fracture in glasses," Appl. Phys. Letters 11, 271-272 (1967).

[10] ASTM Special Technical Publication 469 "Symposium on Damage in Laser Glass," 1969, and references therein.

[11] Giuliano, C.R., Hellwarth, R.W., Hess, L.D., and Rickel, G.R., "Damage Threshold Studies in Laser Crystals," Semiannual Report No. 1 (January 1970) - ARPA Order No. 1434.

[12] Nath, G., and Walda, G., "Strong reduction of laser produced damage in sapphire and ruby by doping with $\mathrm{TiO}_{2}, " \mathrm{Z}$. Naturforsch. 23a, 624-625 (1968).

\section{Ack nowledgments}

We wish to acknowledge the many enlightening discussions with V. Evtuhov and R. W. Hellwarth, helpful comments and suggestions of E.S. Bliss and C.M. Stickley, and the skilled technical assistance of G.R. Rickel. 


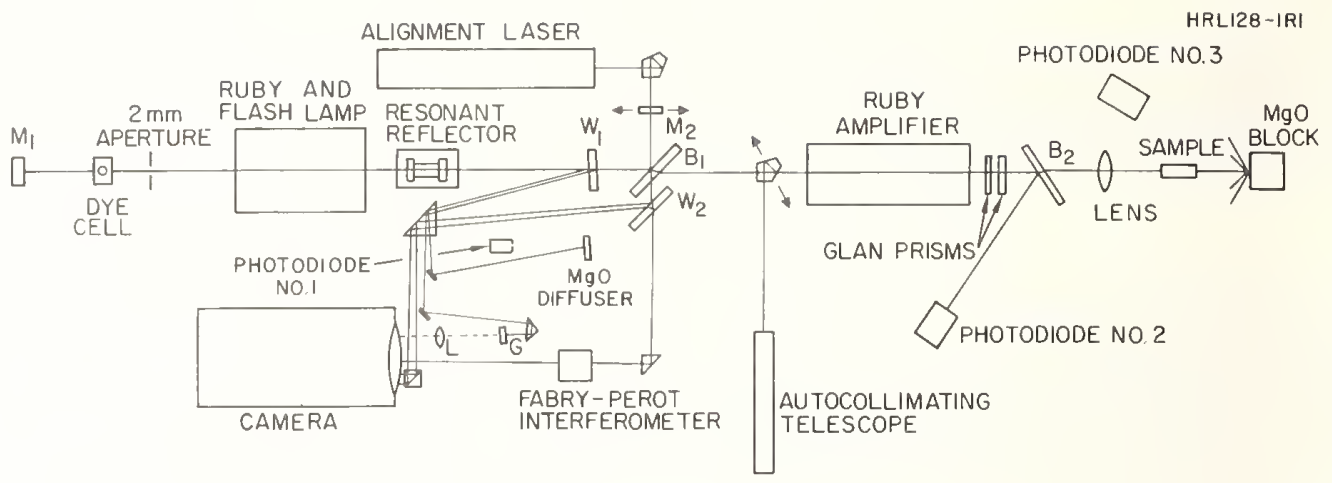

Fig. 1 Schematic representation of experimental apparatus.

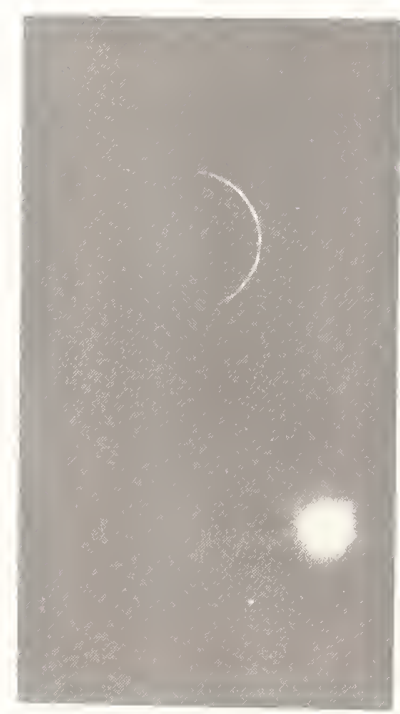

a

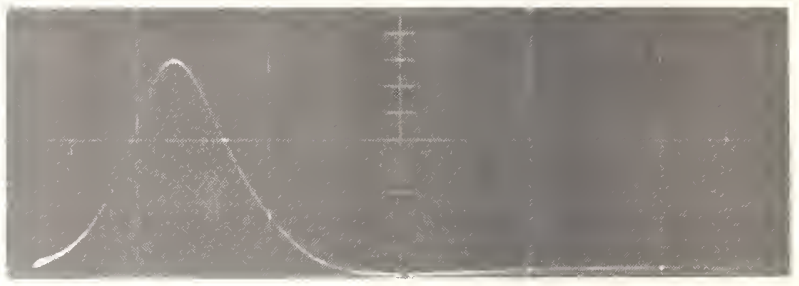

b

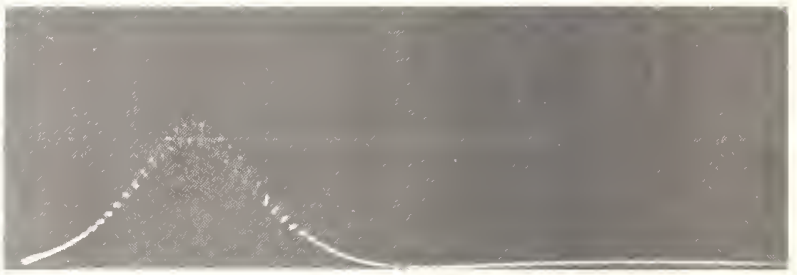

Fig. 3 Tektronix 510 oscilloscope traces of laser output with $20 \mathrm{nsec} / \mathrm{div}$ sweep rate. (a) Smooth pulse observed $\sim 95 \%$ of the time. (b) Modulated pulse $(\sim 750 \mathrm{MHz})$.

Typical photo monitoring FabryPerot interferogram, near-field, and far-field beam patterns. The difference in optical density between the two halves of the picture is N.D. 0.6. The free spectral range of the interferometer is $1.6 \mathrm{~cm}^{-1}$. 


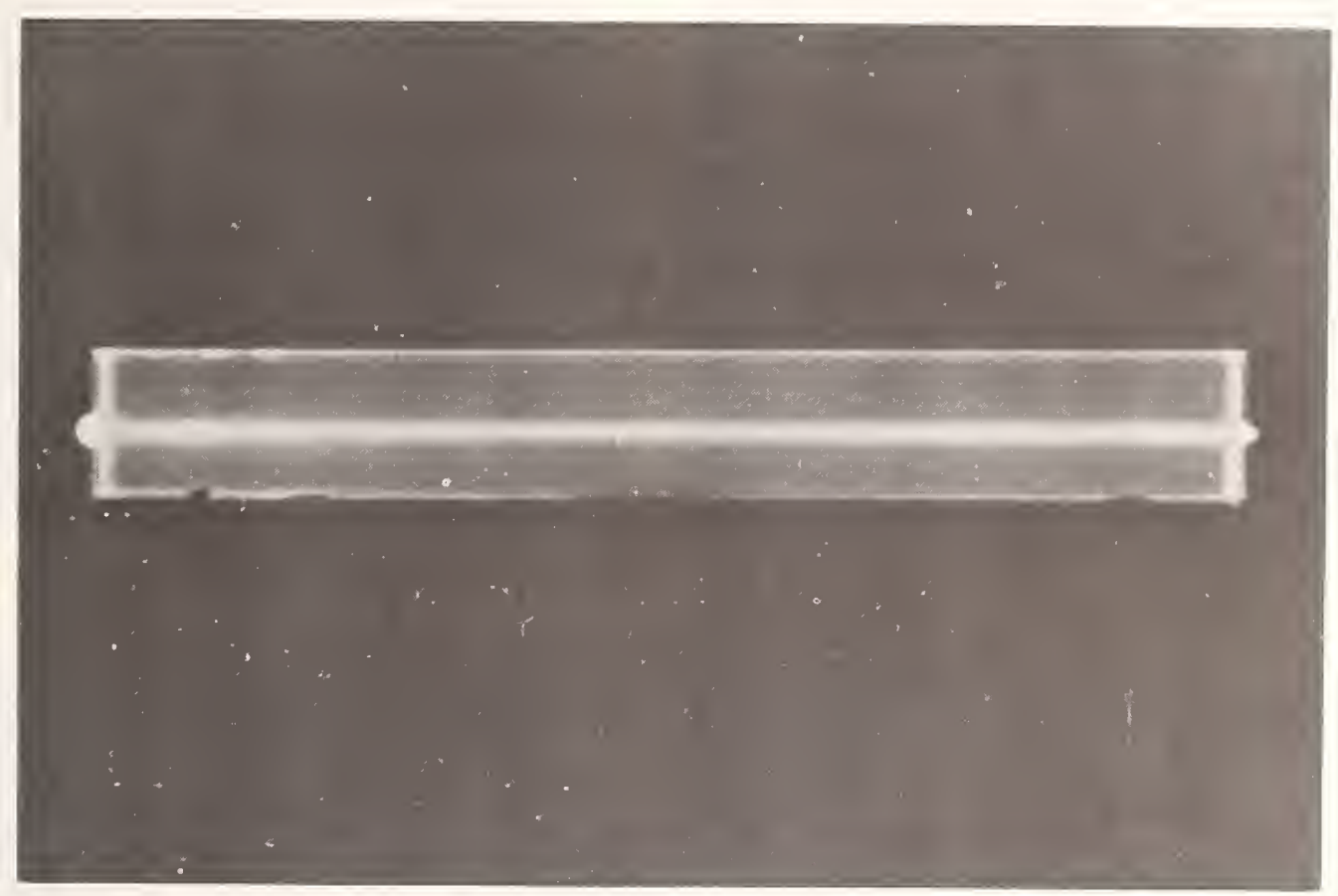

A

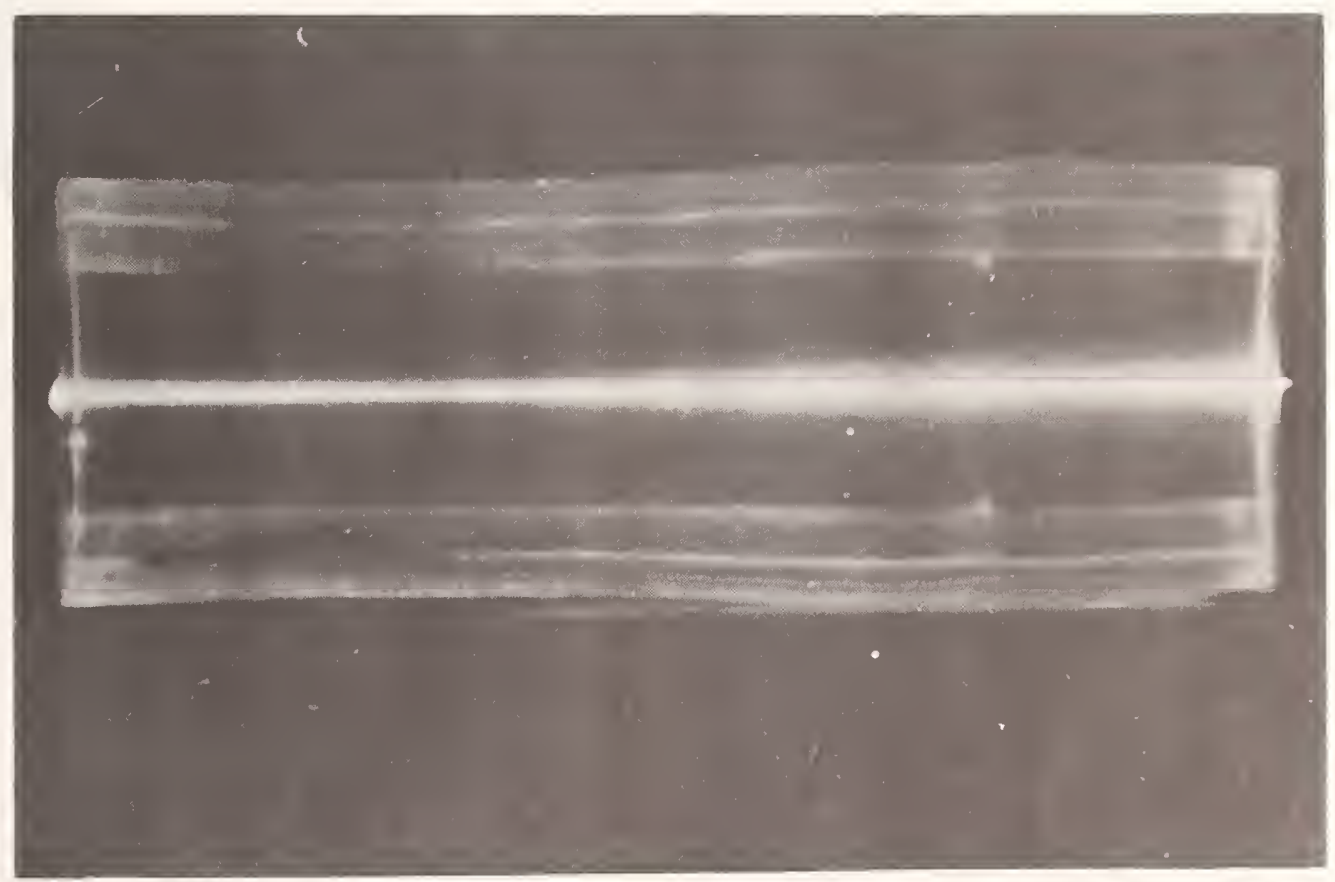

B

Fig. 4 Photographs of ruby samples being subjected to laser radiation. The light is traveling from left to right. Note surface plasma formation. (a) No internal damage. (b) Internal damage plus flaring of beam. 


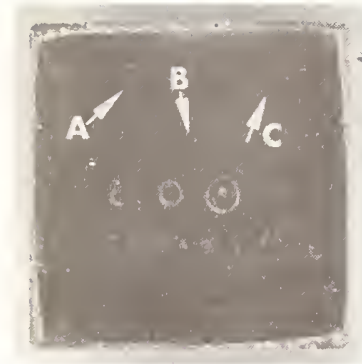

ENTRANCE

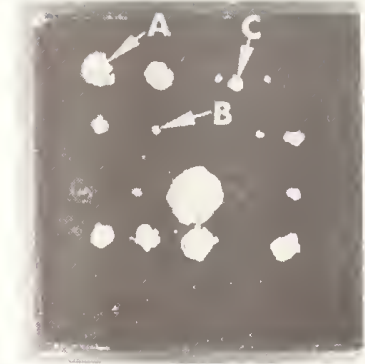

EXIT

Fig. 5 Exit (right) and entrance (left) sur faces of damaged ruby sample ( $1 / 4 \mathrm{in}$. square) after a number of shots. The regions $A, B$, and $C$ are relovant to the discussion in the text.

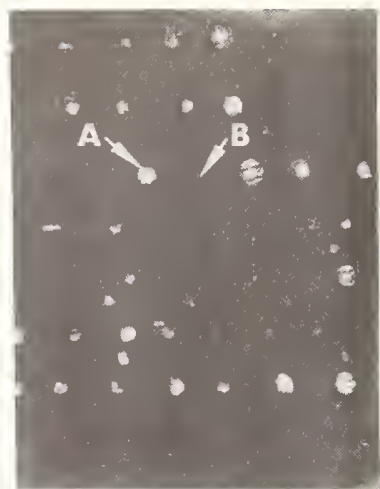

ENTRANCE

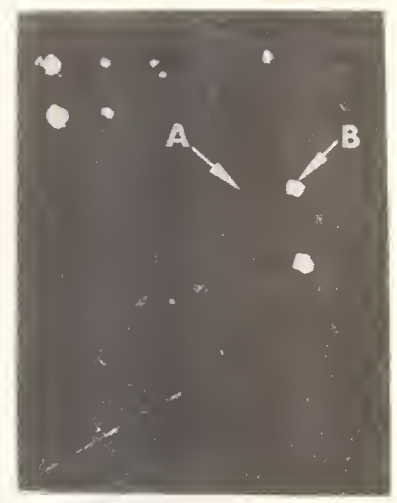

EXIT
Fig. 6 Exit (right) and entrance (left) surfaces of damaged ruby sample ( $3 / 8$ in. square). The regions $\mathrm{A}$ and $\mathrm{B}$ are discussed in the text.

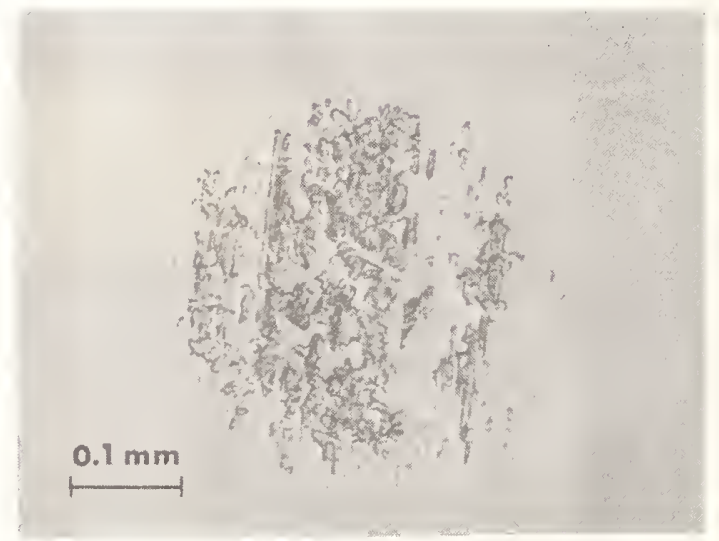

Fig. 7 Magnified view of entrance surface damange asuch as that seen in Figs. 5 and 6.

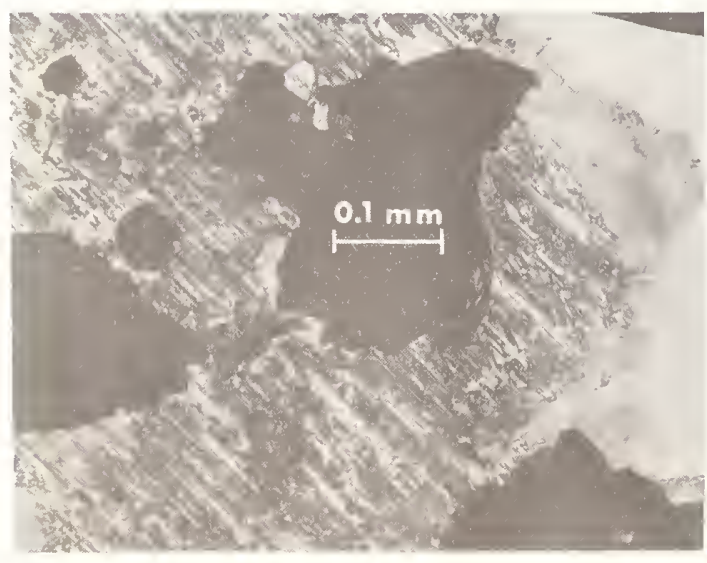

A

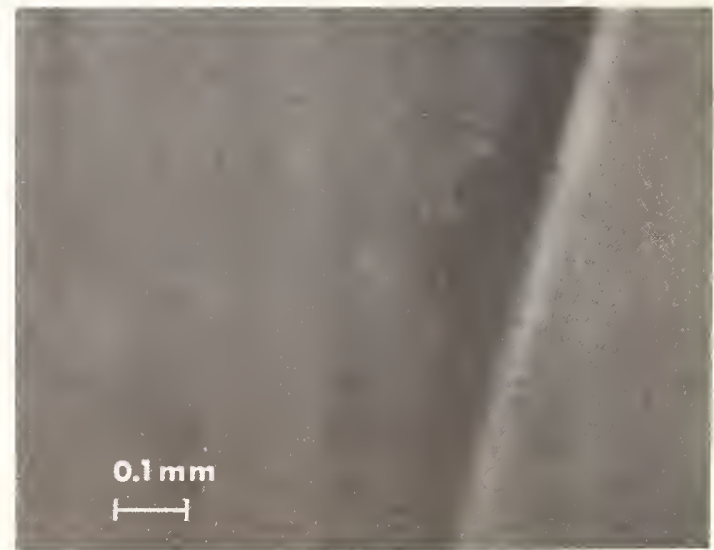

B

Fig. 8. Photomicrographs of exit surface damage pits and crazing. (a) End view. (b) Side view. 


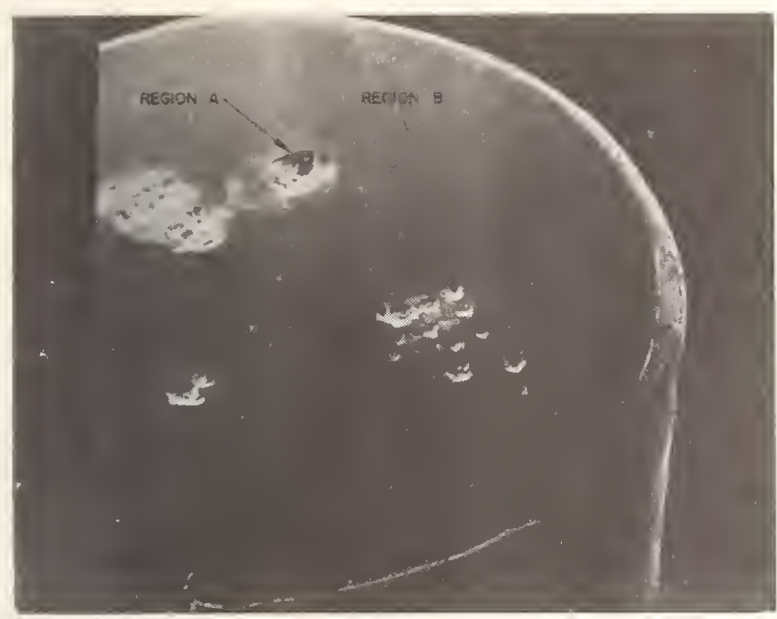

A

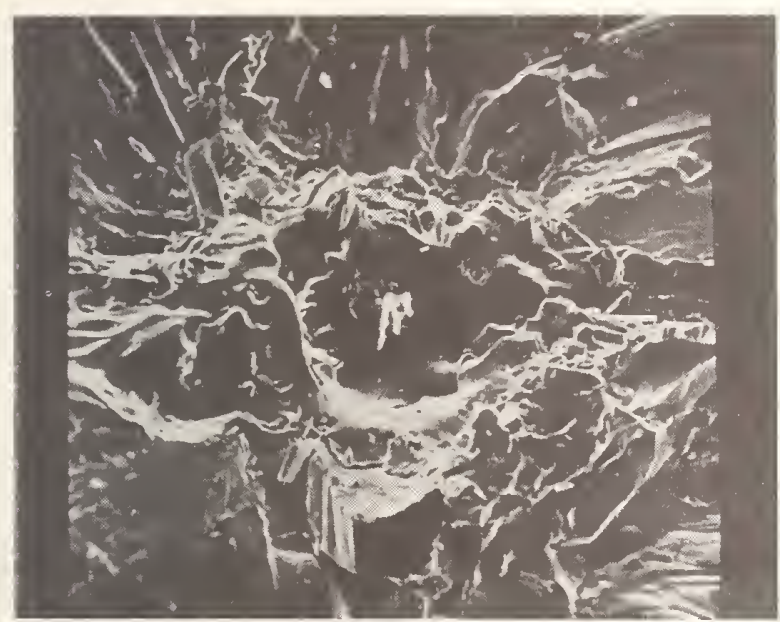

c

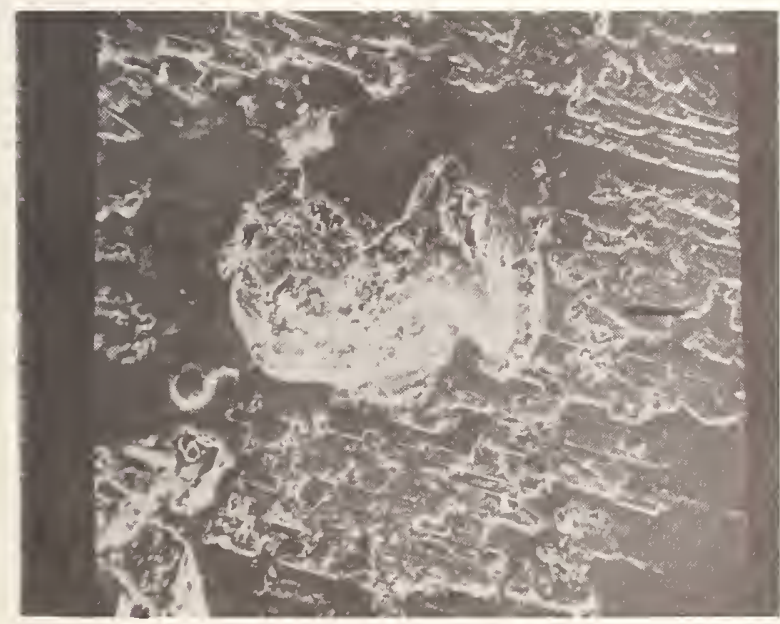

E

\section{B}

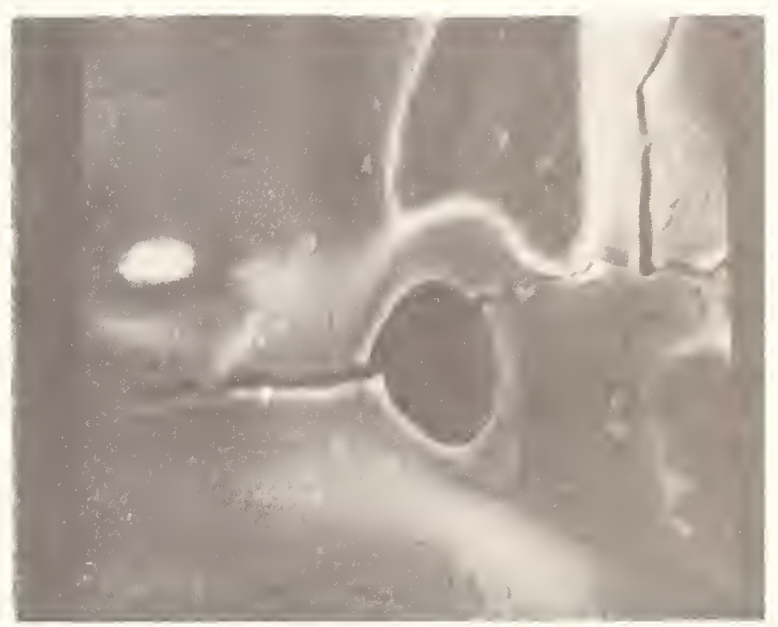

D

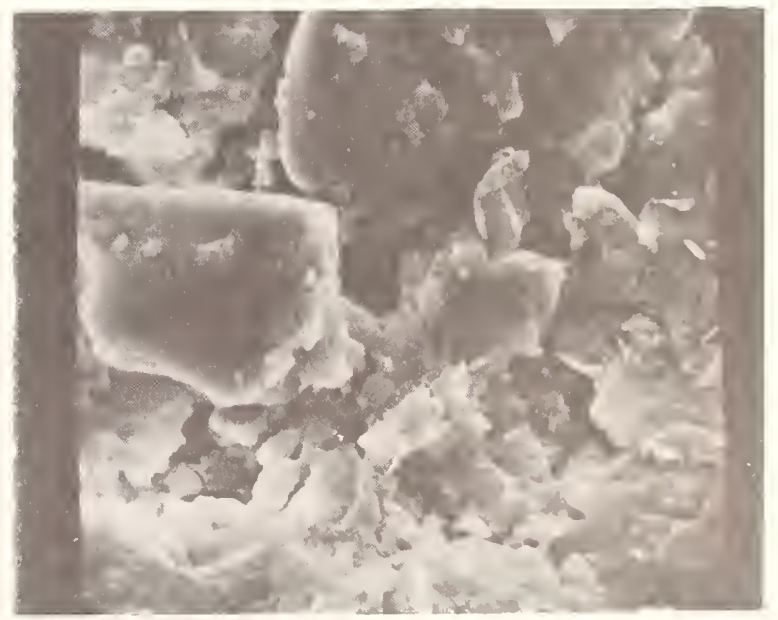

$F$

Fig. 9 Electron micrographs of exit surface damage at different magnifications. (a) End ố ruby rod - Marker length - $400 \mu \mathrm{m}$. (b) Undamaged surface - Marker length - $1.4 \mu \mathrm{m}$. (c) Region A - Marker length - $16 \mu \mathrm{m}$. (d) Region A - Marker length - $1.8 \mu \mathrm{m}$.
(e) Region B - Marker length - $15 \mu \mathrm{m}$.
(f) Region B - Marker length - $1.5 \mu \mathrm{m}$. 


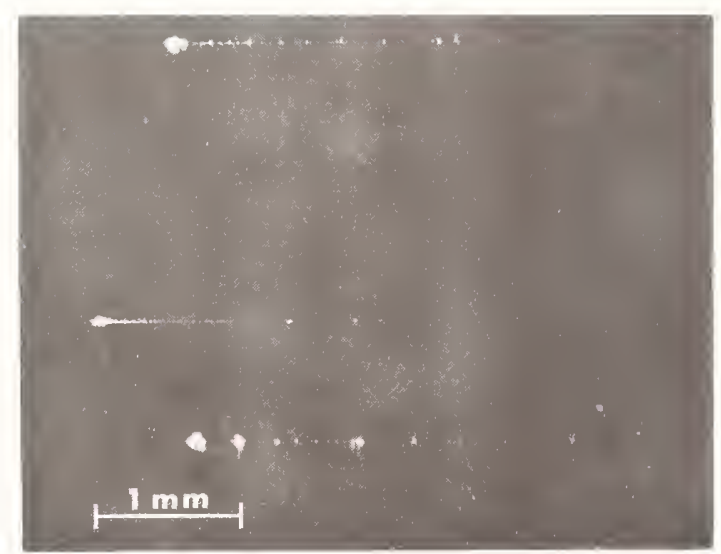

Fig. 10 Photomicrograph of damage tracks in ruby (see text for discussion).

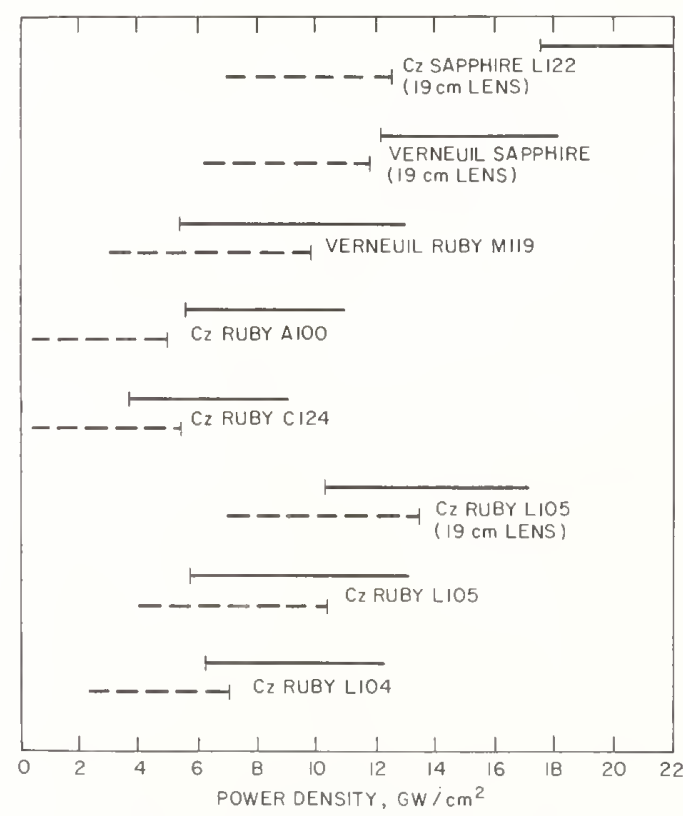

AT BEAM WAIST ( $4 \mathrm{~B} .3 \mathrm{~cm}$ LENS EXCEPT AS NOTEO) (SPATIAL AVERAGE)

Fig. 12 Bulk damage thresholds for a number of ruby and sapphire samples. The power density and energy density scales are spatial averages. The power and energy densities at the peak of the gaussian distribution are twice the spatial averages (see text). The dashed line corresponds to powers where damage was not observed; the solid line to powers where damage was observed for a number of shots in different regions of the same sample. Sample sources: Al00 Airtron; L104, Ll 05, L122 - Union Carbide; C124 - Crystal Optics; M118, Ml19-Meller.

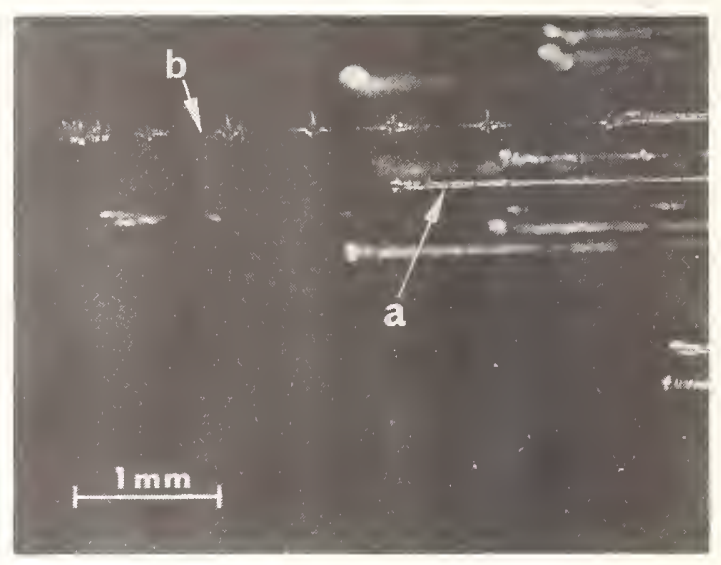

Fig. 11 Photomicrograph of damage tracks in sapphire (see text for discussion).
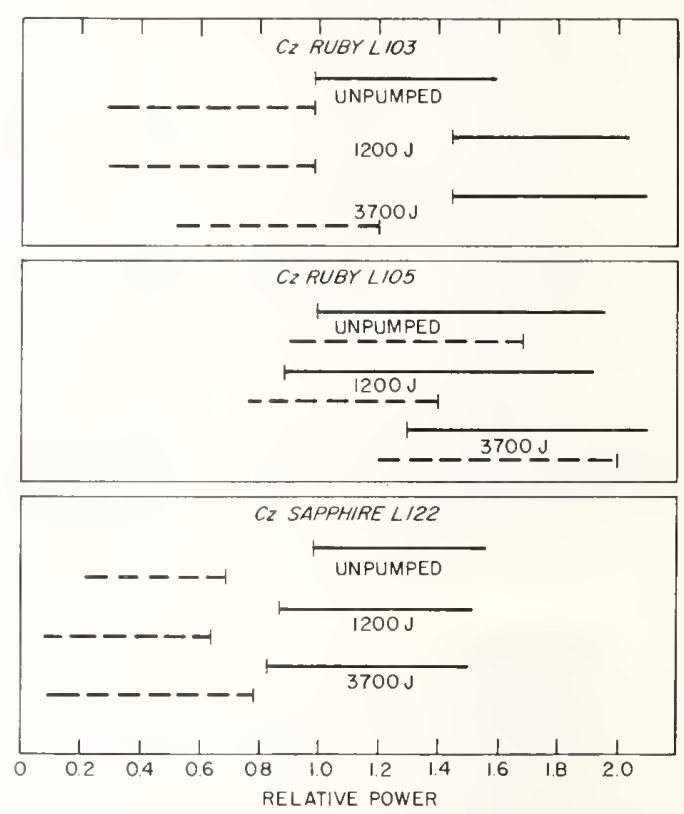

Fig. 13 Relative bulk damage threshold as a function of optical pumping for different samples. The thresholds are normalized to unity for each sample (unpumped). Dashed line - no dam age; solid line - damage. 


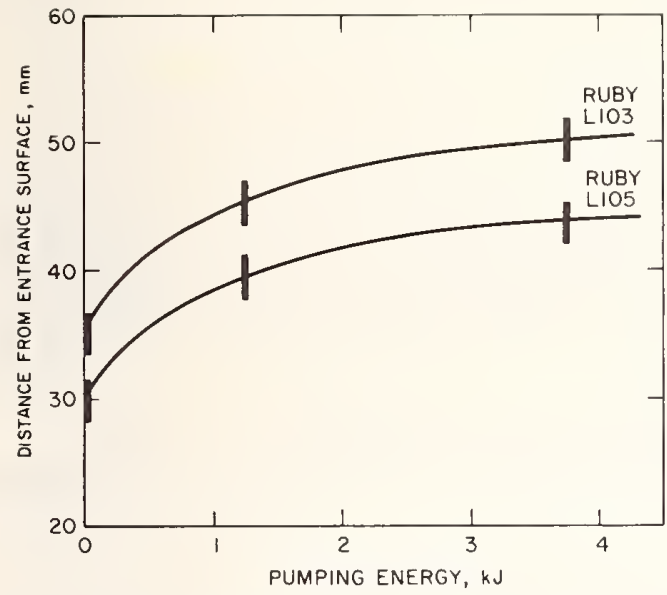

Fig. 14 Location of beginning of damage tracks from entrance surface versus optical pumping energy for ruby samples. $\mathrm{L} 103-0.03 \% \mathrm{Cr}^{+3}$, $\mathrm{L} 105-0.05 \% \mathrm{Cr}^{+3}$.

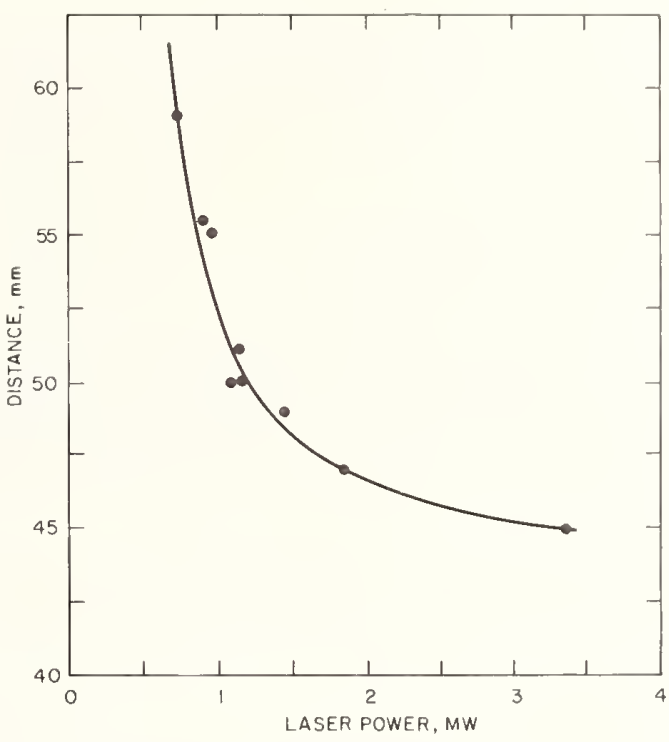

Fig. 15 Location of beginning of damage tracks from entrance surface versus laser power incident on $19 \mathrm{~cm}$ lens for $\mathrm{Cz}$ sapphire sample Ll22.

\section{COMMENTS ON PAPER BY CONCETTO GIUILIANO}

The detailed structure of the "tadpole" shaped damage tracks is characterized by a large, pherical shaped bubble, seemingling away from the direction of incidence of the laser beam. The diameter 10 to 100 wn, extending aw from the bubble. An investigation to determine the presence spacing of these dots increase as was seen by Davit, was not possible, since the requisite instrumentation was not available.

Samples were obtained from manufacturers in the United States, Japan, and Europe, and were oriented in the apparatus without regard to growth direction. No significant dependence of damage threshold on the sample origin was detected. The ruby and sapphire samples used were grown with the long axis of the boule at $60^{\circ}$ to the optic axis. In most tests, the incident laser light was polarized orthogonal to the plane of the c-axis. No polarization dependence of the damage threshold could be detected, due to the considerable scatter of the data points.

In several photographs of the damaged surface, semi-parallel lines were seen to be superimposed on the crazed surface. In general, these lines were perpendicular to the plane of the c-axis of the crystal, and were also oriented parallel to the electric field vector of the incident light. It was noted that this structure is similar in appearance to thermal etching.

Although there is some uncertainty in the interpretation of focused-beam damage levels, the 2 results quoted in this paper correspond to energy densities in the bulk material of 200 joules/cm in 20 nsec pulse, which is an order of magnitude greater than bulk damage levels previously reported in the literature for both Vernewil and Czochraeski rubies.

The occurrence of pitting on the exit faces of samples, with dimensions much smaller than the beam diameter, suggest the possibility of filament formation within the material. If such filament formation did occur, it was not accompanied by catastrophic damage to the bulk material at energy levels at which exit surface pitting was observed. In those cases in which extensive cratering occurred on the exit surface in a single shot, there was evidence of both flow of material and fracture. For the smaller craters, no determination of the presence of flow was carried out.

Data taken in sapphire exhibited a greater variation in damage threshold than that taken in ruby. Dr. Guenther remarked that $0^{\circ}$ sapphire shows a considerable lower damage threshold than $60^{\circ}$ sapphire, from results obtained using sapphire resonators. This could result from differences in the growth processes for the two orientations. The damage observed in resonators made of $0^{\circ}$ sapphire appeared to be filamentary in nature, extending completely through the full thickness of the resonator. 
Laser-Induced Damage to Nonlinear Crystalline Materials;

Michael Bass

Raytheon Research Division

28 Seyon St.

Waltham, Massachusetts 02154

The parameters necessary to specify surface damage to nonlinear crystals are described and the power densities descriptive of the damage process are defined. The definition of the occurrence of surface damage is discussed and preliminary experimental results given for LiNbO 3 , $\mathrm{Ba}_{2} \mathrm{NaNb}_{5} \mathrm{O}_{15}$ and several other materials irradiated by a Q-switched Nd: YAG laser. These results obtained using TEM 00 and "multimode" lasers are analyzed and used to provide the basis for planning future experiments.

Key Words: Damage Threshold, Noninear Optics, Optical Damage, Q-Switched Laser, Second Harmonic. Generation

\section{Introduction}

The essential component in many modulators and Q switches, and in all frequency doublers and parametric oscillators, is an optically nonlinear crystal. Unfortunately, the nonlinear materials having the largest nonlinearities (i.e., linear electro-optic coefficients, second harmonic generation (SHG) coefficients, etc.) are also those most easily damaged by intense laser beams. Even the slightest damage, which can occur on the surfaces or in the volume of the material, can render the crystal useless. Damage to optically nonlinear materials is thus a major limitation in the design of high power lasers and optical devices.

Most reports which describe new optically nonlinear materials mention the occurrence of index-of-refraction gradients when high-average-power visible light passes through the crystal. In $\mathrm{LiNbO}_{3}$ this type of damage is thought to be caused by the presence of crystalline imperfections, possibly oxygen vacancies, which trap electrons. Visible light frees these electrons, which then move away from the imperiection. The resulting internal electric fields cause index-of-refraction gradients throughout the crystal, by means of the linear electro-optic effect. Thus a coherent process such as phase-matched SHG will be severely limited. However, since heating the crystal permits the electrons to return to their initial sites, this type of damage is reversible.

On the other hand, when the light intensity incident on the crystal is high enough, irreversible damage occurs. Because of residual absorption, very high average power beams can heat the material to the point where thermal-stress-induced fracture occurs. The present discussion, however, is concerned with damage caused by very high-peak-power pulses of light to crystals which do not absorb at the optical wavelength. When exposed to such pulses, the crystal's surfaces may become pitted and charred, and/or the interior may become cracked or filled with bubbles. This catastrophic damage can be corrected only by repolishing the surfaces or by replacing an internally damaged crystal. Since in most operational configurations surface damage usually occurs in nonlinear materials atlower power densities than volume damage, it is the first type of damage to be considered.

\section{Parameters Necessary to Characterize Surface Damage}

Since the operational characteristics of all lasers and laser systems vary widely, it is necessary to describe the light pulse responsible for the damage in question. In this manner, meaningful comparisons can be made between different materials and the results of different workers. The relevant properties of light pulses are listed in Table I. The pulse energy must be given in joules rather than some arbitrary relative unit which is meaningful only to the investigator who defines it. It is adequate to give the power density in standard units (MW/ $\mathrm{cm}^{2}$ ) if one also gives the pulse duration and area irradiated. Moreover, the definition of pulse duration (FWHP or the time interval between 10 percent and 90 percent of the total energy, etc.) must be given to permit the pulse power to be computed. Finally, because the pattern of damage pits and the estimate of the power density depend on the mode pattern and size of the beam incident on the crystal surface, these too are essential.

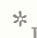

Initially supported in partby the Electronics Research Center under NASA Contract NAS12-2155.

$\dagger$ The research reported in this paper is presently being sponsored in part by the Air Force Cambridge Research Laboratories, Office of Aerospace Research, under Contract F19628-70-C0223, but the report does not necessarily reflect endorsement by the sponsor. 
It is, of course, necessary to specify the state of the surface by giving the parameters isted in Table II. Since contaminants such as polishing compounds and finger grease can lead to spurious conclusions, comments about the cleaning procedure are necessary. In addition, such crystalline properties as hardness or hygroscopic nature are necessary to describe the character of the surface studied.

Having specified the light pulse and the crystal surface, it is now possible to identify the power densities which describe the interaction leading to surface damage. It is intuitively obvious that for very small power densities, no damage occurs. The highest power density of the particular pulse which will never damage the surface is defined as $\mathrm{P}_{\infty}$. It should be noted that, if a system employing this pulse and this crystal surface is to operate successfully and reliably, then the power density on the surface must always be less than $\mathrm{P}_{\infty}$. It is also obvious that a single pulse at some high power density will damage the surface. We therefore define the lowest power density which will damage the surface in a single pulse as $P_{1}$. Note that by definition

$$
\underline{\mathrm{P}_{\infty} \leq \mathrm{P}_{1}}
$$

If $\mathrm{P}_{\infty} \neq \mathrm{P}_{1}$, then for power densities between $\mathrm{P} \infty$ and $\mathrm{P}_{1}$ the number of pulses required to damage the surface, N, will depend on the power density. The form of this relationship is potentially very useful in helping one to understand the mechanism of surface damage.

For a particular light pulse the surface damage properties of a crystal's surface can be characterized by $\mathrm{P}_{\infty}, \mathrm{P}_{1}$, and the relationship between $\mathrm{N}$ and $\mathrm{P}$ for $\mathrm{P}_{\infty} \leq \mathrm{P} \leq \mathrm{P}_{1}$. Since

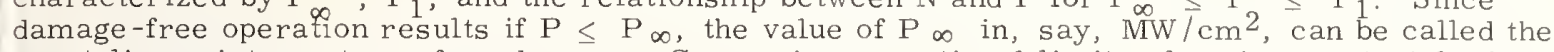
crystal's resistance to surface damage. Comparing operational limits of various materials thus involves determining the values of $\mathrm{P}_{\infty}$

\section{Defining the Occurrence of Damage}

Before damage, the crystal surface, under high magnification, is clean and as free of scratches and pits as possible. When a pulse of light of intensity $>P_{1}$, impinges on a crystalline surface, a spark is emitted as shown in Fig. 1; one or more pits, depending on the transverse mode pattern of the light, are left in the surface. For values of $\mathrm{P}$ in the range $\mathrm{P}_{\infty} \leq \mathrm{P} \leq \mathrm{P}_{1}$, pits can be seen when the surface is viewed with the unaided eye, only after a number of pulses and generally only after a visible spark has been seen. Thus we can say that a surface has been damaged when, after irradiation, one or more pits in the area irradiated can be seen with the naked eye. The possibility exists that very tiny pits do occur before they are visible to the naked eye; this will be studied further in future experiments. However, even using magnifications up to $\sim 200 \mathrm{X}$, such "mini-pits" could not be detected before there were pits which one could see or before a spark was observed. Therefore, since the present definition is a reliable criterion and is functionally easy to apply, it is used in the experiments described below. There is, however, the reservation that future experiments may prove this definition incorrect; in that event the results given at this time might have to be suitably modified.

\section{The Present State of Surface Damage Data for Optically Nonlinear Materials}

There is a paucity of data on this subject. Our experience with the problem extends back about three or four years, to the first $\mathrm{LiNbO}_{3}$ Pockels cells we used in Nd: YAG laser Q-switches. Since this material is not hygroscopic, is harder than KDP, and has large linear electro-optic coefficients, $\mathrm{LiNbO}_{3}$ is an attractive alternate to $\mathrm{KDP}$ in Pockels cell applications. However, as the operating level of a $5 \mathrm{~mm} \mathrm{Nd}$ :YAG rod in a cavity using two flat mirrors is increased to obtain "multimode," 10 nsec pulses of 20 millijoules or more, one or both of the surfaces of the LiNbO 3 crystal become pitted in a pattern characteristic of the most intense regions in the laser beam. An example of this is shown in Fig. 2. This limits the use of $\mathrm{LiNbO}_{3} \mathrm{Q}$-switches to low-power devices; KDP must be used in high-power units.

About one and a half years ago, while designing and building a frequency doubler to operate on pulses with $1.06 \mu$ power densities which could be as high as $10 \mathrm{MW} / \mathrm{cm}^{2}$, it became necessary for us to characterize the doubler crystal's damage resistance as well as its potential as a doubler At the same time, we discovered the nonlinear properties of certain dye crystals; moreover, we found that some of them showed very high resistance to damage by $1.06 \mu$ pulses. This property makes these crystals potentially effective doublers of high-peak-power pulses.

On the basis of nonlinear properties alone, we narrowed the choice of doubler material to "hot- $\mathrm{LiNbO}_{3}$ " or $\mathrm{Ba}_{2} \mathrm{NaNb}_{5} \mathrm{O}_{15}$, assuming both could be obtained in high-optical-quality pieces. Today $\mathrm{LiIO}_{3}$ would also be included. The experiment sketched in Fig. 3 a was used to study the resistance of these materials to surface damage. During these first experiments, we used "multimode" beams, cared little for the orientation of the crystalline axes with respect to the light polarization, and used pulses which varied from $\sim 10$ to $30 \mathrm{nsec}$, depending on operating conditions. The 
pulse energy was varied by varying the laser input energy. We were, however, careful to obtain similar surface quality in all crystals (at least $\lambda / 4$ flat over the small regions irradiated), treat them equally (except for those which were hydroscopic), use a low pulse repetition frequency (i.e. , ppm), and to employ two observers to obtain two independent sets of data. The damage pattern caused by a focused "multimode" laser on a $\mathrm{LiNbO}_{3}$ surface is shown in Fig. 4.

The list of surface damage resistance in Table III was obtained in this initial phase of the work. Clearly the table is incomplete, it represents damage from a "multimode" beam and does not reveal any of the functional dependence of the number of pulses required to damage the surface on the power density. On the other hand, such a list as this is indicative of the differences in resistance to surface damage among nonlinear materials. In addition, since the surface quality of KDP, which has the highest resistance to surface damage, was not as good as that of $\mathrm{LiNbO}_{3}$, this compilation also shows that the differences are caused by the material and not the surface finish.

As we were interested in "hot" $\mathrm{LiNbO}_{3}$ and $\mathrm{Ba}_{2} \mathrm{NaNb}_{5} \mathrm{O}_{15}$, detailed studies of these two materials were initiated. However, since we had no piece of "hot $\mathrm{LiNbO}_{3}$ " which we could sacrifice to damage studies, $\mathrm{LiNbO}_{3}$ was used. * We used samples which were cleaned and polished flat to $\lambda / 4$ to obtain the results shown in Fig. 5 .

The straight-line relation between $\log \mathrm{N}$ and $\log \mathrm{P}$ for $\mathrm{P}_{\infty}<\mathrm{P}<\mathrm{P}_{1}$ shows that for irradiation by pulses such as those which we used, the number of pulses required to damage a surface and the power density are related by an inverse power law. The functional form of $\mathrm{N}(\mathrm{P})$ is shown in Fig. 6. Since the laser output was varied by varying the flashlamp input in these early experiments, it is possible that the laser mode varied in a systematic manner, resulting in these particular relationships. However, the $\mathrm{LiNbO}_{3}$ damage data described below, which was obtained using a TE MOO mode laser, also shows a power law relationship for intermediate values of $\mathrm{P}$ though the value of $\mathrm{m}$ is different.

The values of $\mathrm{P}_{\infty}, \mathrm{P}_{1}$ and $\mathrm{m}$ depend on the beam's transverse mode or, in other words, on the transverse intensity distribution. Using the setup sketched in Fig. $3 \mathrm{~b}$ where the laser was restricted to the $T E \mathrm{M}_{00}$ mode and the power density could be varied without altering either the pulse duration or the polarization state, we found that the value of $\mathrm{P} \infty$ for $\mathrm{LiNbO}_{3}$ is $\approx 350 \mathrm{MW} / \mathrm{cm}^{2}$ as compared to $6 \mathrm{MW} / \mathrm{cm}^{2}$ for a "multimode" pulse. $* *$ In this case, $\mathrm{P}_{1} \cong 1600 \mathrm{MW} / \mathrm{cm}^{2}$. The damage pit on $\mathrm{LiNbO}_{3}$ obtained in a single pulse of $1.06 \mu$ in a TEM 00 mode of $\mathrm{P} \cong 1600 \mathrm{MW} / \mathrm{cm}^{2}$ is shown in Fig. 7. This pit is $\sim 2.7 \mathrm{X} 10^{-2} \mathrm{~mm}$ in diameter and $\sim 2100 \mathrm{~A}$ deep. The volume of the pit depends on the power density in a manner which is presently under investigation.

Figure 8 shows the measured relationship between $\mathrm{N}$ and $\mathrm{P}$ for $\mathrm{LiNbO}_{3}$ when irradiated by the pulsed, Q-switched TEMI 00 mode, Nd: YAG laser shown in Fig. 3 b. The pulse duration was $15 \mathrm{nsec}$ (FWHP) and it was focused to a nearly circular spot of diameter $\leq 3 \times 10^{-2} \mathrm{~mm}$. The crystal surface was polished flat to $\lambda / 10$ and its smoothness figure was $(0,0)$. It was uncoated and cleaned with lens tissue and methanol after each damage spot occurred. During these experiments the crystal was oriented so that the b axis was normal to the surface and the light polarization parallel to the crystal's a axis. Note the similarity in the functional relationships between $N$ and $P$ obtained in the "multimode" and TEMT00 mode experiments.

Determination of the area irradiated proved to be the major source of error in the measured power densities. Several measurements of this quantity were obtained by direct measurement of the area burned by the focused laser beam on the surface of several different materials (stainless steel, anodized aluminum, etc.). The area was also calculated from the beam diameter incident on the objective lens and the effective $f$ number. These various determinations agreed reasonably well with one another, but in order to be conservative and underestimate the power density, we selected the largest value of the area obtained.

The difference between the values of $\mathrm{P}_{\infty}$ and $\mathrm{P}_{1}$ using $\mathrm{TEM}_{00}$ mode and "multimode" beams can be understood by considering that the peak power density in a "multimode" beam (i. e., in each "hot spot") can be many times the value estimated from the pulse energy, its duration and the total irradiated area. In each hot spot, power densities as high as in a TEM 00 mode are possible (see Fig. 4) if at least 75 percent of the "multimode" energy is evenly distributed among the several hot spots. On the other hand, we do not at this time have an adequate explanation of the power law relationship obtained with both types of irradiation. It is possible, however, that the different slopes in Figs. $5 a$ and 8 represent differences due to the orientation of the crystal.

* This was a LINOBATE crystal obtained from Crystal Technology, Ine. Since damage to properties may be related to the crystal preparation procedures samples from other producers will be studied in future work.

***

Note that this is the average power density of the focused $\mathrm{TEMI}_{00}$ mode beam. At the center the peak power density is at least $2 \mathrm{X}$ the given value. 
All the preceding measurements were performed at a pulse-repetition frequency of 1 pps, at room temperature, and in air. No noticeable difference was detected in $\mathrm{P}_{\infty}$ for $\mathrm{LiNbO}_{3}$ when the crystal was heated to $200^{\circ} \mathrm{C}$ or when the ambient atmosphere was $\mathrm{N}_{2}$, Ar or vacuum.

\section{Some Analysis and Future Plans}

We see from the preceding results that the occurrence of surface damage in nonlinear materials has a well-defined threshold; in terms of incident power density, the threshold for surface damage is $\mathrm{P}_{\infty}$. The damage process at power densities above $\mathrm{P}_{\infty}$ appears to involve a localized change on the surface. It might be that microscopic damage spots occur, then grow with successive pulses until they become visible. However, the change in the surface, whatever it may be, accumulates until the last pulse of light strikes the area and causes a breakdown (at this point we usually see a spark) and damage becomes visible to the eye. Determining the nature of the change and how and why it accumulates are major objectives of future damage studies. It is also important to determine the generality of the behavior shown in Figs. 5 and 8 and the effect of environmental conditions and sample treatment on $\mathrm{P}_{\infty}$, and $\mathrm{P}_{1}$, and $\mathrm{m}$ for various materials.

The comparative data on surface damage reveal that the material, and not the surface treatment alone, accounts for the differences recorded. The KDP offers outstanding evidence of this; it cannot be polished finer than $\lambda / 4$ and smoother than $(10,20)$ but the resistance to damage is the highest yet measured. Improvement in surface quality alone then could improve a given material's resistance to surface damage, but it will not eliminate the differences between materials.

Since the surface-quality question inevitably arises, we recently began to examine the imperfection density per unit area of various crystalline surfaces using large angle X-ray scattering. Even the smallest crystalline misorientation, and any cracks, scratches, or other imperfections, show up as areas of different scattering intensity in this type of measurement. Figure 9 shows these Berg-Barrett X-ray topographs of the polished surface of both a $\mathrm{LiNbO}_{3}$ and a $\mathrm{KDP}$ crystal. This procedure enables one to locate surface imperfections and to try to correlate damage pits with these locations. The density of these imperfections per unit area might also be correlated with damage threshold. We shall continue these X-ray measurements in order to explore these possibilities and to provide the requisite data about surface quality.

Future work to determine the causes of surface damage includes:

1) Studies of the dependence of $\mathrm{P}_{\infty}, \mathrm{P}_{1}$, and the relationship between $\mathrm{N}$ and $\mathrm{P}$ on the laser wavelength, pulse duration, temporal properties, repetition rate, and transverse mode pattern. The dependence on crystal orientation, temperature, and environment must also be included.

2) Examination of the surface after each pulse using high magnification microscopes and/or interferometric techniques (possibly holographic correlation methods) and

3) Measurement of changes in the electronic character of the surface.

Various Nd, ruby, and dye lasers and appropriate second harmonics will be used as the sources in these experiments. The data will be analyzed and correlated, if possible, with other crystalline properties such as the location of the band edge, ir absorption bands, and hardness. In this respect, the dye crystals should prove interesting since they will permit us to attempt to relate damage properties to very small changes in chemical composition.

We have, in a manner of speaking, only scratched the surface of this problem. To date we have been able to place limits on the performance of a few materials under certain specific types of irradiation. These measurements for the several most important crystals provide useful design data for high power optical devices employing nonlinear materials. In addition, we hope, through the experiments outlined above, to be able to understand the damage mechanism and perhaps discover procedures to overcome the problem.

\section{Acknowledgements}

The author wishes to thank D. Bua for his invaluable experinental assistance and Dr. R. Mozzi for the Berg-Barrett X-ray topographs. 


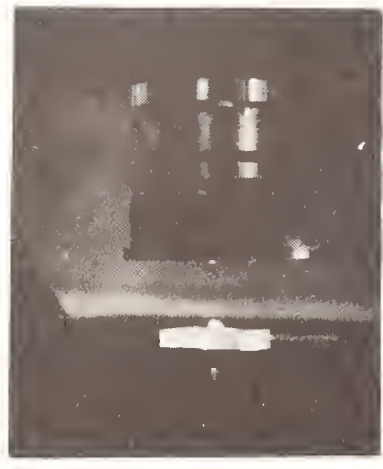

Fig. 1 Spark Emitted by a $\mathrm{LiNbO}_{3}$ Crystal when Irradiated by an Intense $1.06 \mu$ Laser Beam

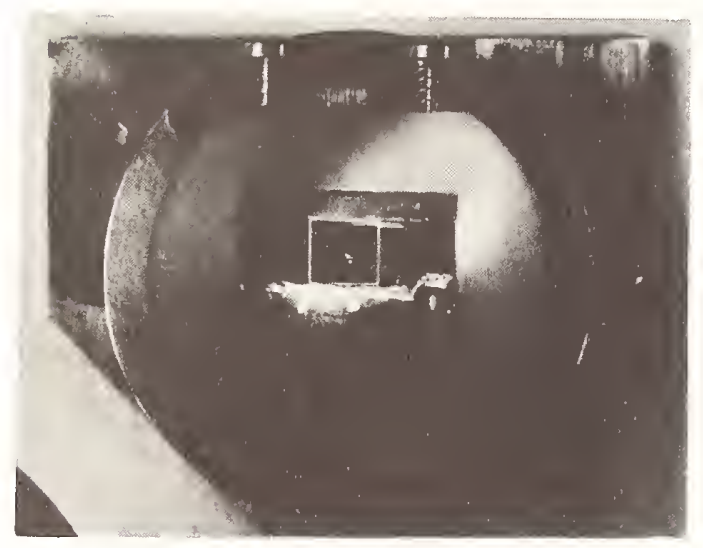

Fig. 2 Damaged $\mathrm{LiNbO}_{3}$ Pockels Cell

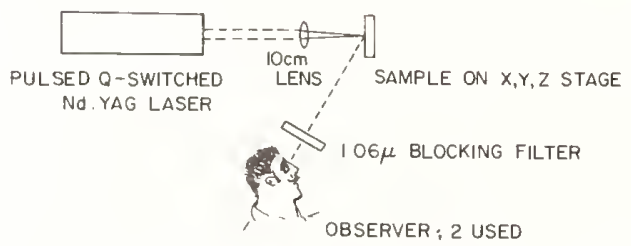

EXPERIMENTAL SETUP; INITIAL PHASE

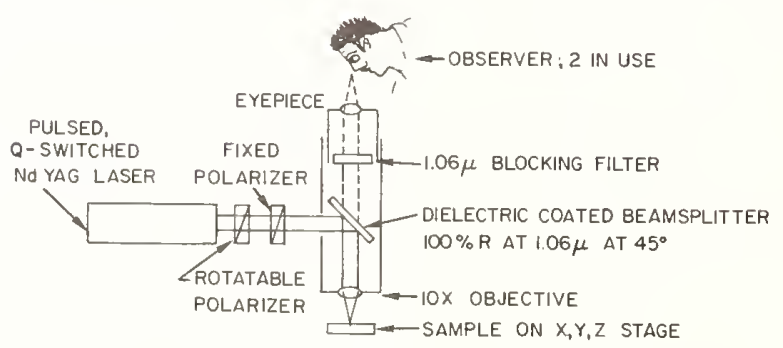

EXPERIMENTAL SETUP; PRESENT PHASE

Fig. 3 Setup for Surface Damage Experiments

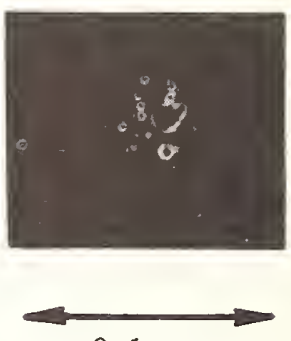

$0.1 \mathrm{~mm}$

Fig. 4 Surface Damage on $\mathrm{LiNbO}_{3}$ Due to One Pulse from a Multimode $Q$-Switched Nd:YAG Laser. Average power density $\sim 250 \mathrm{MW} / \mathrm{cm}^{2}$; dark field illumination.

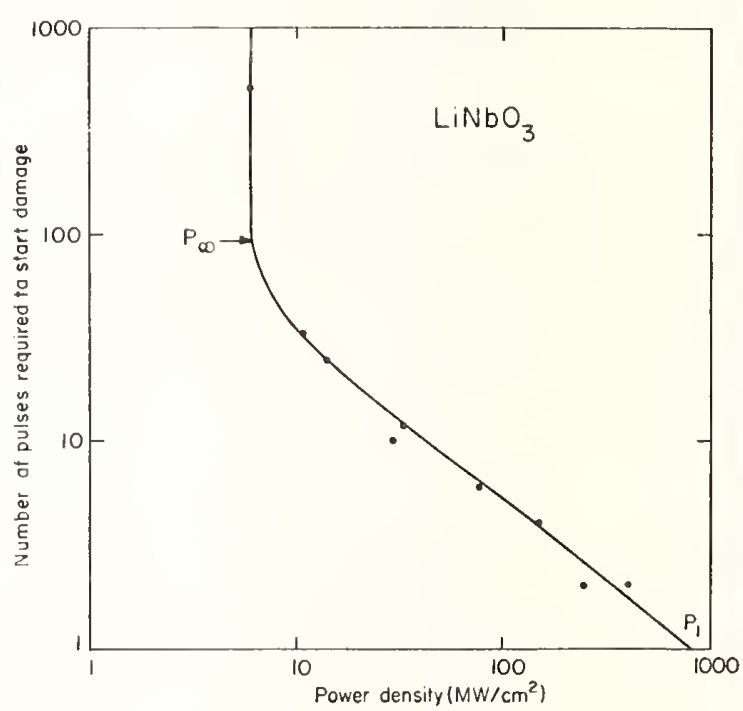

(a)

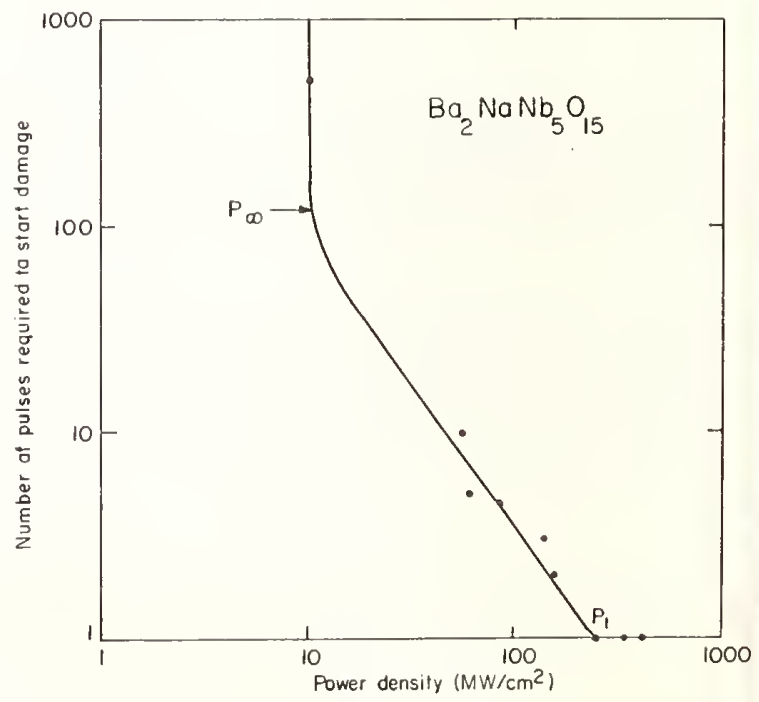

(b)

Fig. 5 Measured $\mathrm{N}=\mathrm{N}(\mathrm{P})$ using Multimode, Pulsed, Q-Switched Nd:YAG Laser 
(1) FOR $P \leq P_{\infty}$

$N \rightarrow \infty$

(2) FOR $P_{\infty}<P<P_{1}$

$$
N \propto P^{-m} \quad \text { AND } N>1
$$

WHERE $m \approx 0.8$ FOR Li NbO$O_{3}$

AND $m \approx 1.3$ FOR $\mathrm{Ba}_{2} \mathrm{NaNb}_{5} \mathrm{O}_{15}$

$2 \mathrm{~mm}$

(3) FOR $P=P_{1}$

$$
N=1
$$

Fig. 6 Functional Form of $\mathrm{N}(\mathrm{P})$ for $\mathrm{LiNbO}_{3}$ and $\mathrm{Ba}_{2} \mathrm{NaNb}_{5} \mathrm{O}_{15}$ with Multimode $1.06 \mu$ Irradiation

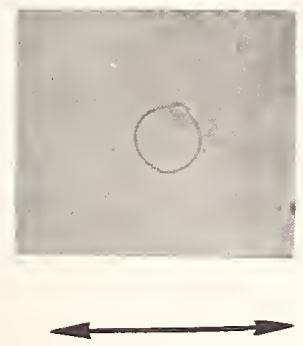

$0.1 \mathrm{~mm}$

Fig. 7 Surface Damage on $\mathrm{LiNbO}_{3}$ Due to One Pulse from a TEM 00 Mode, Q-Switched Nd:YAG Laser. Average power density $\sim 1600 \mathrm{MW} / \mathrm{cm}^{2}$; bright field illumination.

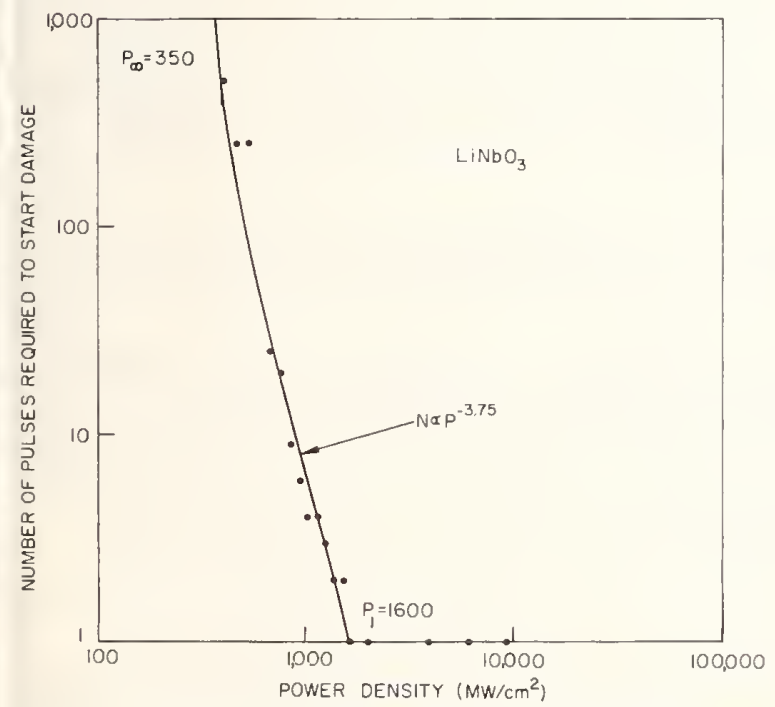

Fig. 8 Measured $\mathrm{N}=\mathrm{N}(\mathrm{P})$ Using $\mathrm{TEM}_{00}$ Mode Pulsed, Q-Switched Nd:YAG Laser

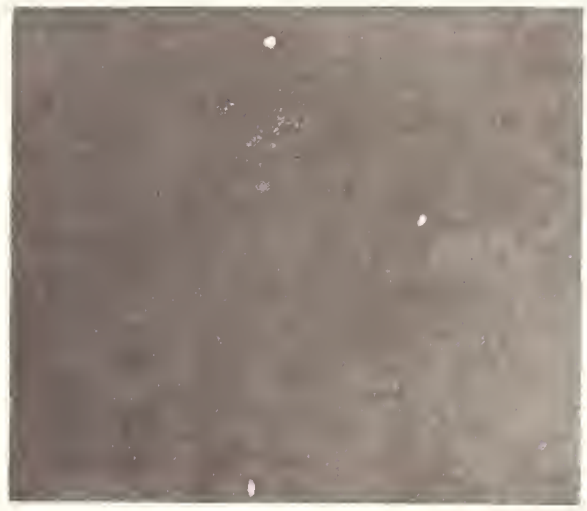

$\mathrm{LiNbO}_{3}$

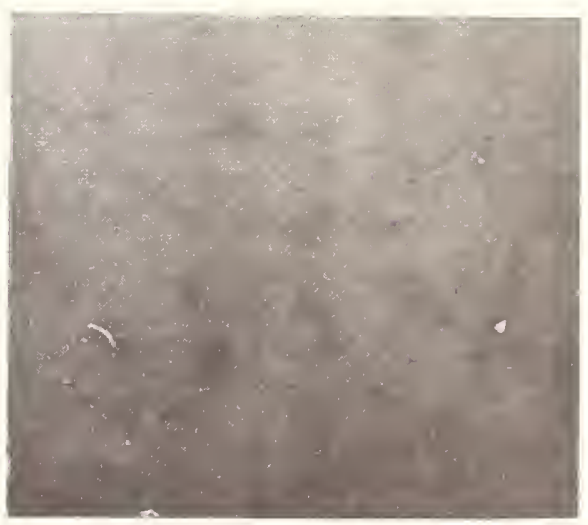

$\mathrm{KDP}$

Fig. 9 Berg-Barrett Surface Reflection X-Ray Topographs of Polished Crystalline Surfaces 
Table I Parameters Necessary to Characterize a Pulse of Laser Light
Table II Parameters Necessary to Characterize a Crystalline Surface

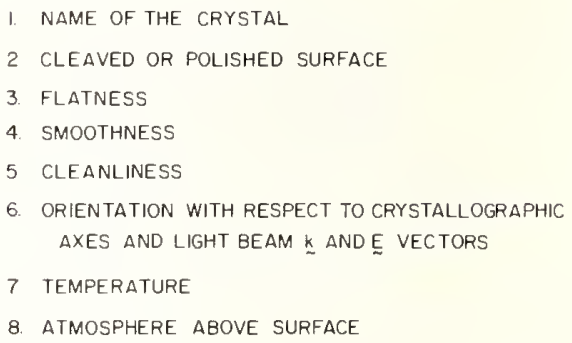

Table III Resistance to Surface Damage for Several Nonlinear Materials

\begin{tabular}{lc} 
& $P_{\infty}\left(\frac{\mathrm{MW}}{\mathrm{cm}^{2}}\right)$ \\
\cline { 2 - 2 } POTASSIUM DIHYDROGEN PHOSPHATE (KDP) & $>400$ \\
AMMONIUM DIHYDROGEN PHOSPHATE (ADP) & $>400$ \\
7 - DIETHYLAMINO- 4-METHYLCOUMARIN & 400 \\
COUMARIN & 10 \\
$\mathrm{Ba}_{2} \mathrm{NaNb}_{5} \mathrm{O}_{15}$ & 10 \\
LiNbO $_{3}$ & 6
\end{tabular}

\section{COMMENTS ON PAPER BY MICHAEL BASS}

A definite onset of damage was always noted in this work. After a large number of shots without visible damage, the simultaneous occurrence of visible and audible plasma formation and a damage pit was detected. There was no dependence of the damage threshold on crystal temperatures, up to $200^{\circ} \mathrm{C}$, or atmospheric environment (including air, argon, nitrogen, and vacuum). All tests were carried out at a repetition rate of one pulse per second. It was assumed that no electronic effects Iphotoelectrons I could accumulate over periods of one sec. Special precautions were taken in the handling of hygroscopic crystals (KDP. ADP) but they were exposed in a manner similar to the other materials. In most tests, the incident laser beam was focused on the entrance face of the sample. When the beam was focused above or below the entrance face, exit surface damage was observed first. In lithium niobate, it appears that there is a measurable dependence of $p_{\infty}$ on the focal length, although this result is not completely verified.

All of these measurements were made using a focused laser beam of good uniformity I TEH mode). Dr. Guenther pointed out that care must be exercised in extrapolating focused-beam values for damage thresholds to geometries (areas) of larger dimensions.

In measurements performed at Sylvania, reported by Dr. Fountain, a crystal of lithium niobate was immersed in Dow Corning 200 bluid, and irradiated with an intense diverging beam created by jocusing the incident YAG laser beam above the crystal with a $20 \mathrm{~cm}$ focal length lens. In all cases, damage was seen at the exit face of the crystal. When the crystal was immersed in the Dow fluid, the threshold for plasma formation is reduced by a factor of three. In addition, if the crystal of lithium niobate is not present, breakdown is not observed in the Dow fluid. The inference is made that an acoustical effect plays a role in the plasma formation. An additional result reported by Dr. Fountain is that if one places quartz in optical contact with the surface of the crystal, the damage threshold increases by a factor of four.

Dr. Giuliano commented that in experiments he had carried out, the spectrum of the plasma formed at the surface of various materials was characteristic of the material itself, and independent of the atmospheric environment. 
David F. Edwards ${ }^{1}$, C. Y. She ${ }^{2}$, J. D. Masso ${ }^{3}$, Y. D. Harker ${ }^{4}$ and H. C. Schade 2

Quantum Electronics Laboratory

Colorado State University

Fort Collins, Colorado 80521

Raman scattering is being used to study microscopic damage in crystalline quartz. Three experiments have been performed to date. The Raman spectrum of undamaged quartz was measured to serve as a standard from which the criteria for microscopic damage may be defined. A controlled defect experiment was next in which was measured the effect of an applied uniaxial stress on the Raman spectrum. A modified-valence force model was developed to calculate the stress dependence of the Raman shift for the $A_{1}-v i b r a-$ tions. The agreement between the observed and calculated values is satisfactory. Quartz samples were damaged by a Q-switched ruby laser and changes in the Raman spectrum were observed and are reported here.

Key Words: Applied stress, laser damage, linewidth, microscopic damage, precatastrophic damage, quartz, Raman scattering.

This is a report of work we have been doing in the Quantum Electronics Laboratory of Colorado State University. We have been studying laser produced microscopic damage in crystalline materials and glasses.[1]5 By microscopic damage, we mean damage that is not visable under ordinary visual examination. Microscopic damage also could go by the name of precatastrophic or fatigue damage that accumulates from shot-to-shot until some threshold is reached and then cracks or "bubbles" appear within the crystal.

The primary objective of this research has been to make an experimental and theoretical investigation into the criteria governing the onset of damage and of the associated mechanism or mechanisms by which the damage is produced by laser radiation in transparent solids. We are using light scattering as the diagnostic tool since the type of defect or inhomogeneity that we are searching for has the dimension of the order of the wavelengths of light.

Quantitative Raman scattering is being used as the diagnostic tool for our experiments, that is, not only do we measure the line position but we also measure the linewidth and the line intensity. The binding or interatomic forces in solids are dependent on the electronic structure surrounding the atoms. The application of a uniaxial stress, a temperature change, or an intense laser pulse might be expected to change the structure in a crystal and thereby alter the interatomic forces. This alteration might be indicated either by the production of a Local-phonon mode, or by a change in the characteristic frequency of a vibrational mode, a change in the phonon lifetime and thus a change in linewidth, or the induced electronic polarizability might be effected causing a change in the line intensity. Each of these effects (i.e., the Raman line position, width, and intensity) were measured fcr various Raman lines in quartz in an attempt to measure the effects of microscopic damage. Quartz was selected because it is a crystalline material and has physical properties similar to those of glass. Investigations similar to those reported here for quartz have also been made on sapphire and ruby but will not be reported here.

* This work was sponsored in part by a contract with the Air Force Cambridge Research Laboratories, Office of Aerospace Research, USAF.

1 Electrical Engineering and Physics Departments.

Physics Department.

4 Present address American Optical Company, Keene, N.H.

5 Present address Idaho Nuclear Corp., Idaho Falls, Idaho.

Figures in brackets indicate the literature references at the end of this paper. 
We have performed three experiments; the first was to determine the quantitative Raman spectra of undamaged quartz, the second was a controlled defect experiment, and the third was to examine laserproduced damage.

In the first experiment a detailed measurement was made of the quantitative Raman spectra of undamaged quartz. We carefully measured the line position, linewidth, and line intensity of each of the Raman-active vibrational modes in the quartz sample. The quantitative spectra of undamaged quartz serve as a standard from which the criteria for microscopic damage may be defined. The samples were of natural and artifically grown material and from our measurements no differentiation could be made as to the source of the material. From these experiments the components of the Raman scattering tensor were evaluated and the effects of the long-range electric-dipole forces were determined. The details of this work are reported elsewhere. [2]

The next experiment was to measure the change in the Raman spectra produced by a controlled defect or inhomogeneity.[3] Starting with an undamaged quartz sample, it was placed between the jaws of an hydraulic press and pressure was applied; the quantitative Raman spectra was measured as a function of the applied uniaxial stress. We measured the line position, linewidth, and line intensity for some of the strong Raman-active vibrations. Given in Fig. 1 is a plot of the experimental data showing the shift in phonon frequency as a function of applied stress for three of the four $A_{1}-$ or symmetic vibrations. The 207- $\mathrm{cm}^{-1}$ vibration is the soft mode of quartz and is associated with the $\alpha-\beta$ phase transition. The $466-\mathrm{cm}^{-1}$ line is the most intense vibration. It should be noted that the $352-\mathrm{cm}^{-1} 1$ ine is essentially stress independent. In Fig. 2 are given the experimental data of the changes in the linewidth (FWHM) of the same three $A_{1}$-vibrations. Here one can see that the linewidth of the 466-cm-1 line is only slightly affected by the applied stress while the linewidth of the $207-\mathrm{cm}^{-1}$ soft mode has the greatest stress dependence. A calculation was made of the stress dependence of the line position for the 4 A 1 -vibrations. The valence-force model of Kleinman and Spitzer [4] was modified to include the effects of an anharmonic potential and an applied stress. Using this model the microscopic or internal strains produced by the atomic displacements necessary to minimize the deformation energy were calculated following the procedure of Born and Huang. [5] The resulting stress-dependent potential, including the anharmonic contributions, was calculated in terms of the normal-mode coordinates of the Al-modes given by Kleinman and Spitzer. The chances in the normal-mode frequencies produced by this stress dependent potential were evaluated using first-order perturbation theory and the results are given in Table I. The details of these calculations can be found elsewhere.[6] Within the accuracy of the calculations and measurements the agreement between the predicted values and the measured values is quite good. The stress dependence of the line position of the $1081-\mathrm{cm}^{-1}$ line was not measured because it was too weak, however, we did calculate a negative slope. One should notice that for these four A 1 -vibrations, (symmetric vibrations) the stress dependence is not monotonic as measured and predicted. Also given in the table are the slopes as measured by Asell and Nico [7] for applied hydrostatic pressures. One can see that within the large error that they quote our measurements are in very good agreement.

Table 1. Comparison of the calculated and observed values of the slope of the frequency shiftstress dependence for the $A_{1}$-modes in quartz.

\begin{tabular}{|c|c|c|c|}
\hline $\begin{array}{l}\text { Mode } \\
\qquad\left(\mathrm{cm}^{-1}\right)\end{array}$ & $\begin{array}{l}\text { (Slope) } \\
\mathrm{cm}^{-1 / \mathrm{kbar}} \text { lc. }\end{array}$ & 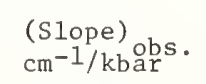 & $\begin{array}{l}\text { (s1ope) (a) } \\
\mathrm{cm}^{-1 / \mathrm{kbar} s .}\end{array}$ \\
\hline 207 & .33 & $1.28 \pm .06$ & 1.6 to 2.1 \\
\hline 352 & .02 & $-0.05 \pm .06$ & -0.2 to +0.3 \\
\hline 466 & .13 & $0.44 \pm .12$ & -0.2 to 0.4 \\
\hline
\end{tabular}

(a) Ase11 and Nicol, reference [7] hydrostatic pressure.

The effects of the applied stress on the line position and width are similar to that observed for decreasing sample temperature. The increase in frequency as the sample is compressed is what might be expected since the atoms become more closely packed making it more difficult for each atom to move. For no change in sample temperature, the increase in phonon frequency would lower the associated phonon population number. Since the phonon life-time depends on the number of phonons available for the multiphonon interactions, a general reduction in phonon population numbers would result in lengthened phonon life-times and consequently narrowed Raman lines. In addition and perhaps more important than the popu- 
lation effect, the phonon lifetimes are also influenced by different multi-phonon interactions resulting from the strain. [8]

Shown in Fig. 3 are spectra of the stress dependence of the line shape for the $128-\mathrm{cm}^{-1} \mathrm{E}-\mathrm{vibration}$ Although this line was not used for the studies reported here the stress dependence is typical for the other vibrations. Here is a slight dependence on stress can be seen for the line position, however, the change in linewidth is not discernible from the raw data. Upon deconvolution of this raw data with the instrument function the brodening effects of the spectrometer can be subtracted. When this is done one can easily see not only the change in line position under the applied stress but also the change in width such as given in Figs. 1 and 2 .

In the third experiment the change in the Raman spectra was measured in a quartz sample before and after irradiation with a commercial Q-switched ruby laser pulse structured with multi-transverse modes. Starting with an undamaged sample the Raman spectra was carefully measured in the region of the $207-\mathrm{cm}^{-}$ modes. The sample was then placed in the beam of a pulsed laser having a peak power of the order of 1 MW unfocused, and a power density of about $100 \mathrm{MW} / \mathrm{cm}^{2}$ for a focused beam. No microscopic or visable damage was observed within the sample after the laser pulse. The sample was then placed in the spectrometer and again the Raman spectra was measured for these two lines. This experiment was repeated for various peak powers and power densities for the pulsed laser. Shown in Fig. 4 is a schematic diagram for one of the samples measured. This particular sample contained a seed vein and the relative orientations of the incident radiation and polarization directions are indicated. For this particular measurement a small pit or crater was produced on the exit face of the sample. The Raman spectrum in the region of the 466- $\mathrm{cm}^{-1}$ line is shown in Fig. 5. At the bottom is shown the Raman spectrum for an undamaged sample indicating the strong $466-\mathrm{cm}^{-1}$ line with a peak intensity of about 7,000 counts and a FWHM line width of $6.4 \pm 0.1 \mathrm{~cm}^{-1}$. For the damaged sample containing the seed vein the spectrum is that shown in the middle. Here one sees not only the 466- $\mathrm{cm}^{-1}$ line with a peak intensity of about 4 , 000 counts and a line width of $6.7 \pm 0.1 \mathrm{~cm}^{-1}$, but also new lines at $540-\mathrm{cm}^{-1}$ and $579-\mathrm{cm}^{-1}$. In order to check whether these new lines are due to laser-damage or due to the seed, a sample containing no seed vein previously damaged for our preliminary study [1] was examined in the same spectral region and results were shown at the top. Here new lines at $540-\mathrm{cm}^{-1}$ and $579-\mathrm{cm}^{-1}$ are observed indicating that the $540-\mathrm{cm}^{-1}$ and $579-\mathrm{cm}^{-1}$ lines are probably due to the defects or local phonon modes induced by the laserdamage.

Our immediate goals are to continue this study to try to determine the effects of polarization and direction of propagation of the damaging laser beam on the change in linewidth of the existing lines as well as the creation of new lines. The transverse dimensions of the damaged region are being determined by measuring the Raman spectra of the sample at different distances from the position where the damaging laser was incident.

Two different Q-switched ruby lasers are being used for damaging the quartz samples. For one laser no attempt has been made to eliminate hot spots in the beam. The other laser has been carefully constructed such that no hot spots exist in the beam; the output is a TEM mo mode with a Gaussian shape. Preliminary results indicate that for the laser pulse having the hot spots the average power density necessary for producing damage is significantly lower than that required for the TEMoo pulse. Also the crater produced by the two lasers are noticably different. This work is continuing.

The authors wish to acknowledge the assistance of Dr. G. McAllister and Mr. V. Draggoa for their assistance in helping us perform some of these experiments.

\section{References}

[1] Edwards, D. F., Harker, Y. D. Masso, J. D., and She, C. Y., "Diagnostics and Evidence of Pre-catastropic Damage in Transparent Solids," ASTM STP469, 1969, p. 128.

[2] Masso, J. D., She, C. Y., and Edwards, D. F., "Effects of Inherent Electric and Anisotropic Forces on Raman Spectra in $\alpha$-Quartz," Physical Review B, May 15, 1970; She, C. Y., Masso, J. D., and Edwards, D. F., "Raman Scattering by Polarization Waves in Uniaxial Crystals," Journal of the Physics and Chemistry of Solids, submitted for publication; Masso, J. D., "Raman Scattering in $\alpha$-Quartz," Ph.D. Thesis, Colorado State University, March, 1970, unpublished.
[4] Kleinman, D. A., and Spitzer, W. G., "Theory of the Optical Properties of Quartz in the Infrared," Physical Review, Vol. 125, 1962, p. 16.

[5] Born, M. and Huang, K., "Dynamical Theory of Crystal Lattices," Clarendon Press, Oxford, 1954.

[6] Harker, Y.D., "Effect of Uniaxial Stress on the Vibrational Modes in Quartz," Ph.D. thesis, Colorado State University, December, 1969, unpublished. 
[3] Harker, Y. D., She, C. Y., and Edwards, D. F., "Stress-dependent Raman Frequency and Linewidth in a-Quartz," Applied Physics Letters, Vol. 15, 1969, p. 272; "Effect of Uniaxial Stress on the Vibrational Modes in Quartz," AFCRL-69-0445, October, 1969, "Raman Spectra of a-Quartz Under Uniaxial Stress," Journal of Applied Physics, submitted for publication.

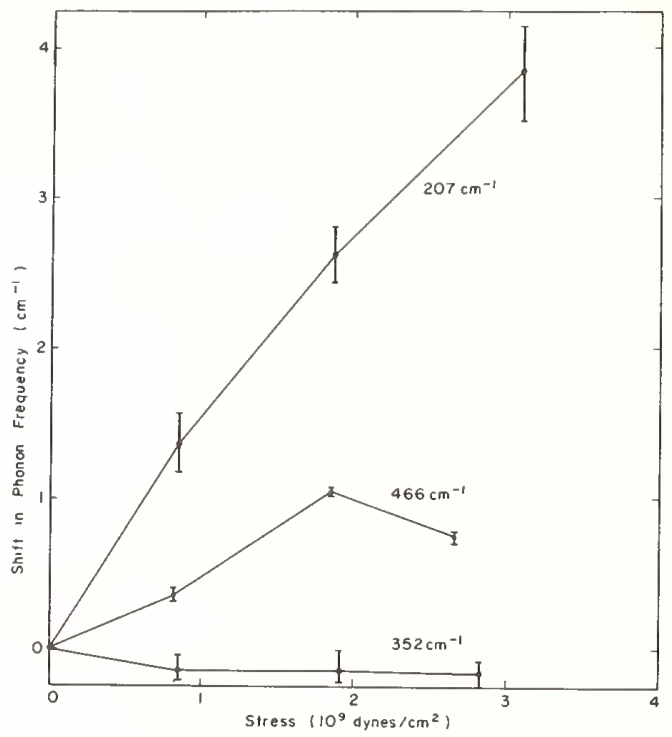

Fig. 1 Shift in phonon frequency as a function of applied stress for three $A_{1}$-vibrations.

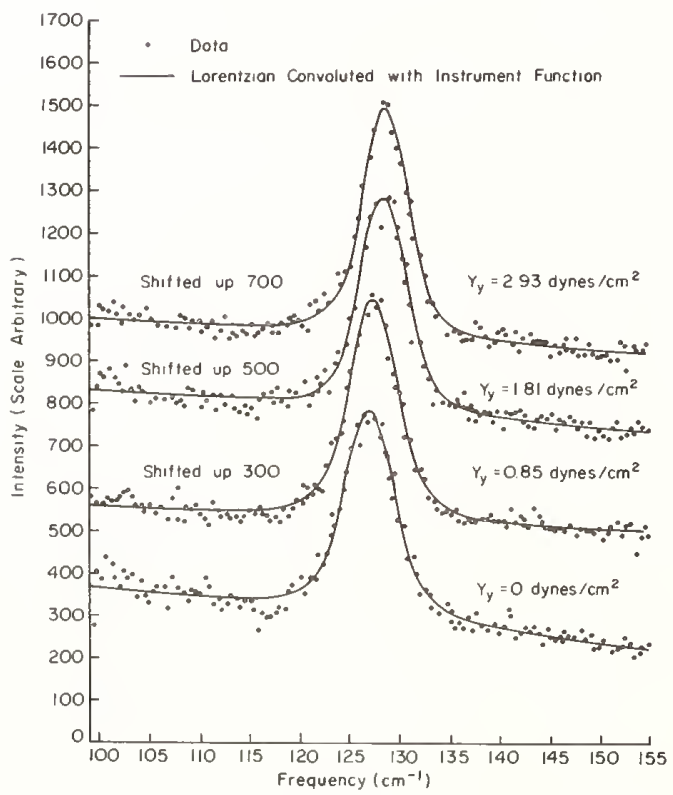

Fig. 3 Stress dependence of the $128-\mathrm{cm}^{-1}$ line
[7] Asell, J. F., Nicol, M., "Raman Spectrum of $\alpha-$ Quartz at High Pressures, "Journal of Chemical Physics, Vol. 49, 1968, p. \$395.

[8] Cowley, R. A., "Phonons in Perfect Lattices and in Lattices with Point Imperfections," edited by R. W. H. Stevenson, Plenum Press, New York, 1966.

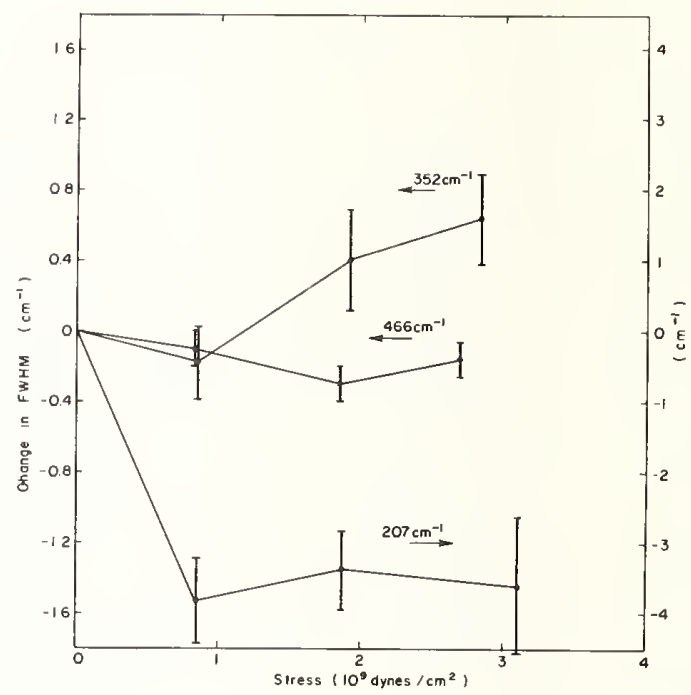

Fig. 2 Change in fullwidth-half maximum as a function of applied stress.
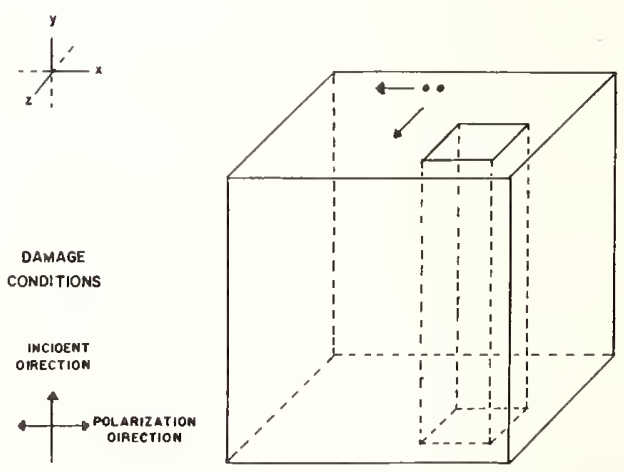

SCALE 20.1

Fig. 4 Schematic diagram of damaged quartz sample showing damaging laser and seed vein orientations. 


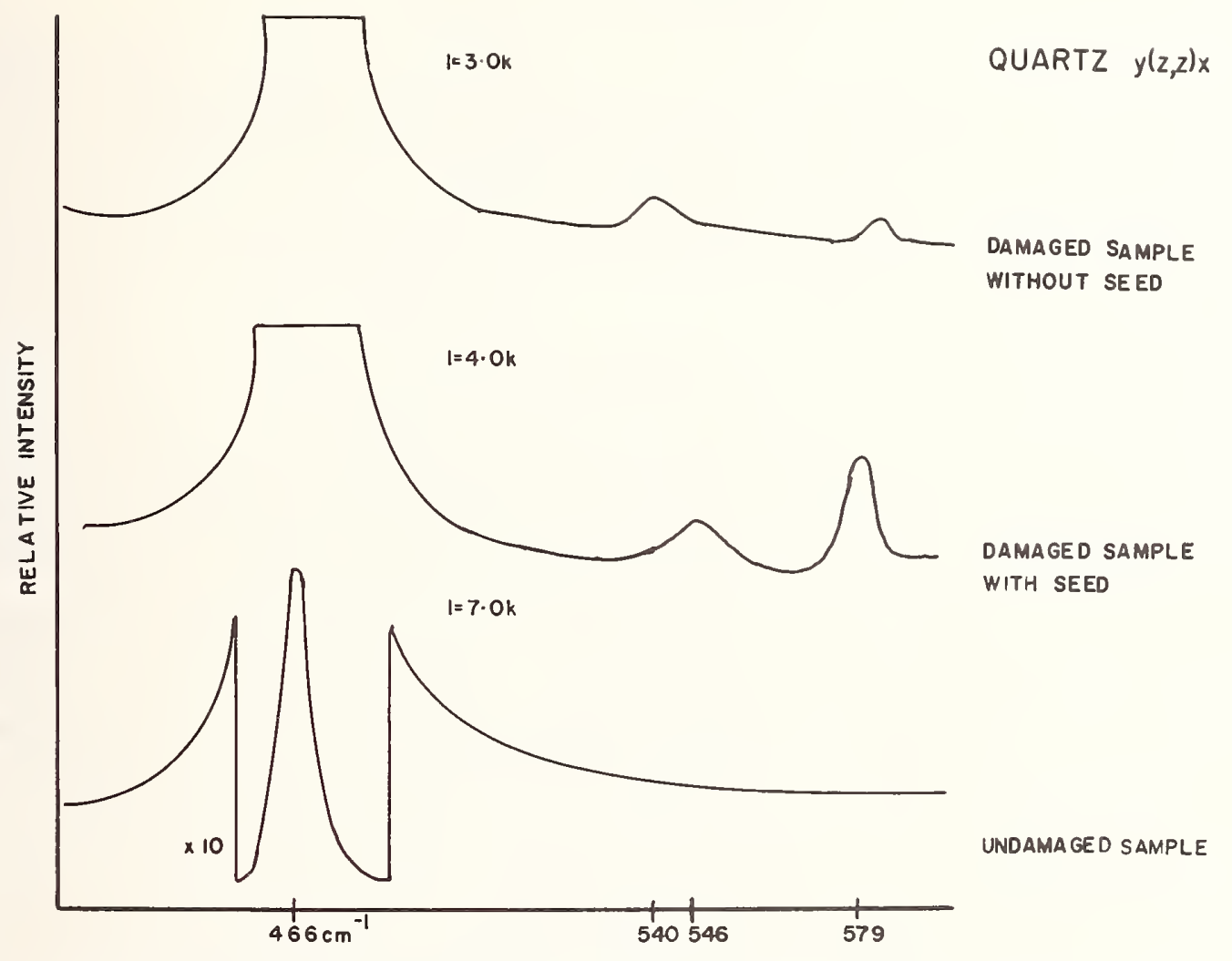

Fig. 5 Raman spectrum in the region of the $466-\mathrm{cm}^{-1}$ line for undamaged and laser damaged quartz samples . 
Exoelectron Emission from Optical Surfaces

P. Bräunlich

Bendix Research Laboratories

Southfield, Michigan 48075

The first exoelectron images obtained from dielectric materials are reported. Possible applications of the new technique in characterizing a surface with respect to laser damage are discussed.

Key Words: Electron emission, work functions, electron imaging, glass surface damage.

\section{Discussion}

Laser surface damage is a serious problem wherever high power laser beams are used. Optical materials such as glass, sapphire, ruby, single crystals of alkali halides, etc., and non-1inear optical crystals such as lithium niobate, barium sodium niobate, lead molybdate, etc., are damaged at various densities. Surface damage usually occurs at lower power densities than damage of the bulk.

The mechanism for this surface damage is presently not wel1 understood.[1] ${ }^{1}$ one fact, however, appears to be established: the condition of the surface is the most important factor in determining the surface damage threshold of a particular material. In most cases, an increase in photoemission. of electrons seems to preceed surface damage.[2] All the materials mentioned above have a wide bandgap Multi-photon absorption of electrons from the valence band or from defect levels below the Fermi level is therefore the obvious mechanism of generating photoelectrons at typical laser wavelengths. In the case of single-photon absorption, photoemission from defect levels plays only a minor role. The quantum efficiency in this case is typically three orders of magnitude smaller than the efficiency of emitting electrons that originate in the valence band.[3] Because the minimum energy required to emit an electron from a defect level is smaller than the energy required to emit electrons from the valence band, electrons from defect levels become important contributors to multi-photon photoemission and can even dominate the total emitted current. Defect levels can also influence photoemission indirectly through the production of band bending. [3] The type of bending that takes place is determined by the position of the Fermi level in the bulk of the material and by the energy available in the surface. It may increase or decrease the minimum energy required for an electron to escape across the work function barrier at the surface.

Obviously, information on the defect levels, the surface states, and the potential barrier of the solid-gas interface is needed in order to estimate the vulnerability of the entire surface or of only certain "weak" areas to high intensity light fluxes. Detailed studies of photoconductivity appear to be the natural source for this information. Unfortunately, the experiments that have to be performed are fairly involved. Multi-photon photoemission requires intense light sources, and surface damage is again likely to occur. Single-photon photoemission, on the other hand, requires light sources emitting in the vacuum uv. This requirement causes detection problems, because most electron detectors (e.g., open electron multipliers) are also photoemitters in the vacuum uv, thereby contributing sizable background problems. Furthermore, the quantum efficiency of photoemission of electrons from defect levels is so small that the investigation of this contribution to the photoeffect is fairly difficult. Thus, a more direct experimental approach to the problem is clearly desirable. In this paper we suggest using the technique known as exoelectron emission (EEE)[4] to study the various aspects of laser surface damage.

After having been exposed to ionizing radiation, many dielectric materials emit electrons from their surfaces. The initial emission decays after a certain time; however, further heating of the compound causes additional electrons to be emitted (EEE). Usually emission peaks at various temperatures are observed, which are characteristic of a specific material and its surface condition. Figure 1 shows a typical EEE curve obtained with LiF that had been exposed to 10 rad $\beta$-rays of a Sr $90-Y^{90}$ source prior to heating. [5]

1 Figures in brackets indicate the literature references at the end of this paper. 
The mechanism of EEE is believed to be the escape of electrons from traps in the surface across the work-function barrier. The traps are formed by crystal defects, adsorbed layers of impurities, etc. The EEE curve of some compounds is remarkably similar to the so-called thermoluminescence glow curve; in these cases, electron traps in the bulk of the sample appear to be the origin of the emitted electrons. The mechanism by which the electrons are released or expelled through the surface is not clearly understood.

The connection between surface damage threshold and EEE seems obvious: the condition of the surface layers is the determining factor for both. Furthermore, the defect level structure and the work function are important in explaining both phenomena.

For the study of EEE, relatively simple experimental techniques are needed. The detection of exoelectrons requires a sensitive counter without a window. Gas-flow Geiger counters, "point counters!" and proportional counters are frequently employed.[5] For studies on a surface in high or ultra-high vacuum, "open" electron multipliers are used.[4] Our preliminary experiments on ED-2 1aser glass showed that Bendix Channeltrons ${ }^{\circ}$ in various configurations are ideal detectors for exoelectrons. Their input aperture can be selected for diameters from 0.001 inch to over 1 inch. It is therefore possible to either scan the surface of the sample or collect the electrons emitted from the entire surface. The quantum efficiency for electrons is close to $100 \%$ and typical gains are $10^{7}$ to $10^{8}$. An example of such a device is shown in Fig. 2. Yet another Channeltron device, the microchannel plate, even permits imaging of the sample via the emitted exoelectrons. With this device, we have been able to demonstrate EEE-imaging for the first time.

The microchannel plate is a bundle of closely packed Channeltron multipliers having a limiting resolution of 0.002 inch (see Fig. 3). Its physical dimensions are typically about 1 inch diameter and 0.080 inch thickness. Electrons emitted from various points on the surface of the specimen are collected by the bundle and multiplied. The electron beams emerging from the channels are accelerated in a dc electric field and then made visible with the aid of a phosphor screen. The gain of these bundles is presently about $10^{3}$. Other similar devices are available that have gains up to $10^{6}$. The dark current is very low and was never found to distrub the imaging in any way. In order to demonstrate the principle of exoelectron imaging, we have performed some preliminary experiments on cleaved samples of LiF single crystals which were subjected to various surface treatments. The images obtained may be interpreted as maps that display the features of the work function, of the defect leve1 distribution, and of certain macroscopic defects across the surface.

A typical EEE image is shown in Fig. 4. A $10 \mathrm{~mm} \times 10 \mathrm{~mm}$ sample of $1 \mathrm{~mm}$ thickness was cleaved from a high-quality pure LiF single crystal. It exhibited two pronounced cleavage steps (35 $\mu$ and $50 \mu$ height) across its center. In order to fill the electron traps, the sample was exposed to X-rays (50 kV, $15 \mathrm{~mA}$, tungsten anode) for four hours. After that, one half of the crystal was etched for one minute in a $3 \% \mathrm{HF}$ solution, which causes only slightly visible changes in the appearance of the surface. The etching demonstrated that different surface treatments distinctly influence EEE images. With the aid of two steel brackets, the sample was then mounted on a heatable copper block inside a vacuum chamber. For good thermal contact, an $\mathrm{Al}_{2} \mathrm{O}_{3}$-compound having a low vapor pressure was applied between the copper block and the crystal. The channeltron bundle was placed $5 \mathrm{~mm}$ in front of the crystal and parallel to its surface. The temperature was then raised within a few minutes to about $200^{\circ} \mathrm{C}$. During the appearance of the $150^{\circ} \mathrm{C}$ peak (see Fig. 1), time exposures of the image displayed on the phosphor screen were recorded with a camera.

The EEE images obtained exhibit a complex structure. At this point, we shall not attempt to interpret this structure other than pointing out that the exoelectron emission from the etched part appears to be distinctly different from that of the rest of the surface. The cleavage steps are clearly visible. Since many other features could not be related to any visible structure of the crystal surface, it seems that not only macroscopic defects, but also other defects and "invisible" property differences of various parts of the surface influence exoelectron emission.

It is hoped that EEE techniques will provide a tool for studying certain surface properties of dielectric solids, as well as the much needed means for characterizing and predicting the quality of optical surfaces with respect to their laser damage threshold.

\section{Acknowledgment}

The author greatly appreciates the assistance of $W$. Colson and $R$. Hall in performing the experiments. 
1. Damage in Laser Glass, ASTM Special Publication, 469, 1969.

2. Glass, A. J. and Guenther, A. H., Review in Damage in Laser Glass, ASTM Special Publication, 469, 1969.

3. Larch, S., ed., Photoelectronic Materials and Devices, van Norstrand, N.Y., 1965.

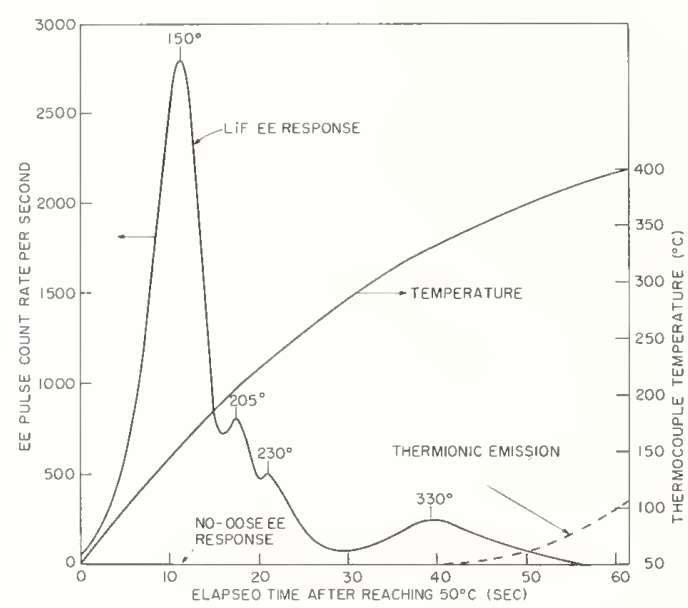

Fig. 1 Typical EEE count-rate versus heating time for a $10 \mathrm{mg}$ LiF sample (powder) measured with a methane gas flow counter after exposure of the sample to $10 \mathrm{rad} \beta-$ rays from a $\mathrm{Sr}^{90}-\mathrm{Y} 90$ source. (The author is indebted to Dr. F. Attix for making the figure available for this publication.)

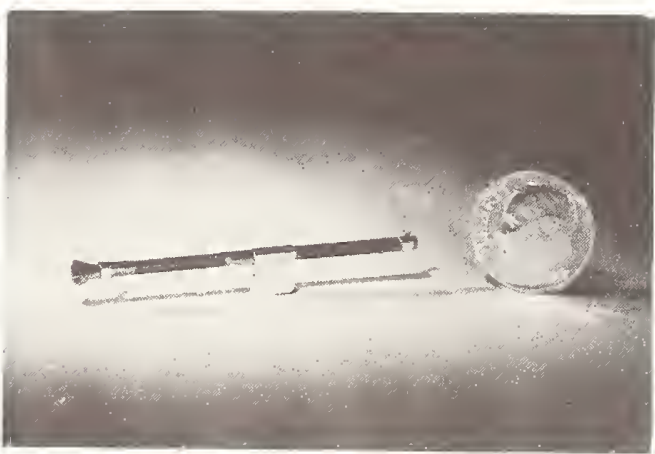

Fig. 2 Channeltron electron multiplier.
4. Scharmann, A., article in "Festkorperprobleme VI" (Vieweg und Sohn, Braunschweig, 1966).

5. Attix, F. H., private communication (1970).

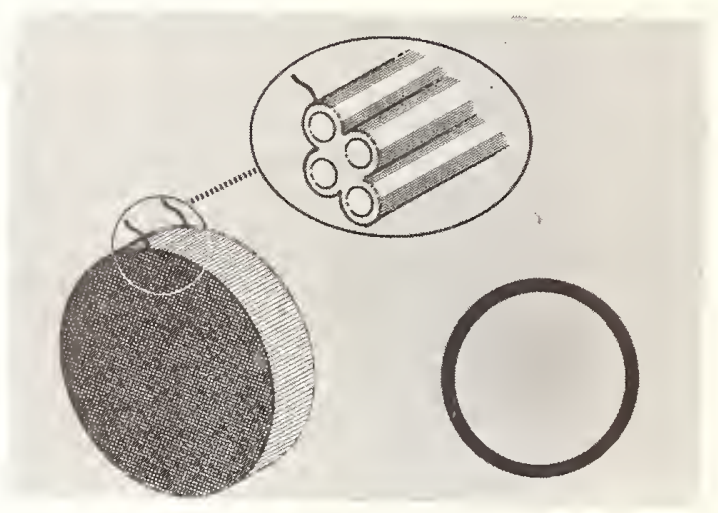

Fig. 3 Channe1tron Arrays,

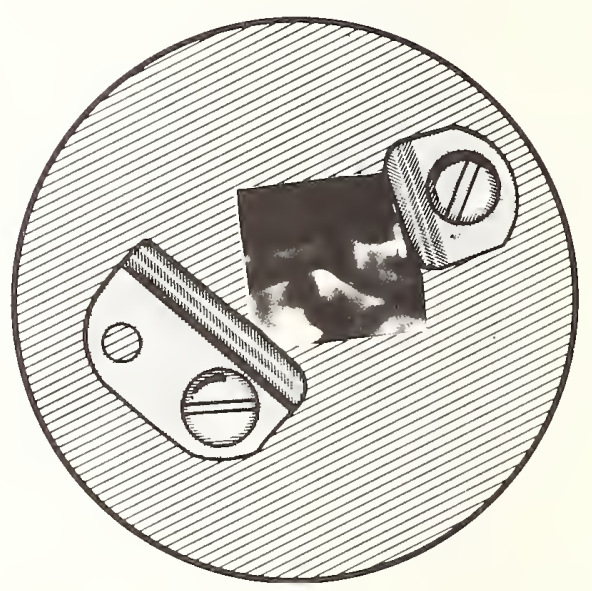

Fig. 4 EEE-image of a LiF crystal. The schematic shows how the sample was mounted on the copper block. The left half of the crystal was etched in a $3 \% \mathrm{HF}$ solution after the $\mathrm{X}$-ray exposure. This part appears brighter (see especially upper left corner). 


\section{E. S. Bliss}

Air Force Cambridge Research Laboratories Bedford, Mass. 01730

A number of mechanisms for laser damage in transparent dielectrics a re examined for pulse duration dependence and the results are compared with experimental measurements when possible. The differences in dependence on pulse duration and other variables from one mechanism to another suggest that different mechanisms may initiate damage in different parameter ranges. Experimental identification of these mechanisms will be aided by measuring the pulse duration dependence of the damage threshold.

Key Words: Conduction electrons, dielectric breakdown, inclusions, laser damage, laser glass, ruby, self-focusing.

\section{Introduction}

It has become clear that damage thresholds under both ruby and glass laser radiation are influenced by a large number of variables. For example, temperature [1]1, pulse duration [2], optical pumping conditions 2 , beam diameter [3], beam focusing [3], laser frequency [1], and details of material growth and preparation [4,5] all appear to be significant in particular instances of laser damage. This multiplicity of variables often makes it difficult or impossible both to compare various experimental findings with each other and to understand their relation to a particular damage theory or to an operational laser system. A plot of damage threshold versus pulse duration from data available in the literature, for example, reveals considerable scatter. This probably results from significant variations in other important parameters from one experimental arrangement to another.

Various theoretical efforts have been directed toward detailed studies of individual damage mechanisms, but only rarely has an attempt been made to explicitly predict how the damage threshold might vary over a wide range of a given system or material parameter. The purpose of this paper is to indicate what pulse duration dependence is expected for a number of laser damage mechanisms. Information about dependence on other important variables is essential in some cases and is included for completeness. When possible these predictions are compared with experimental measurements. However, only a few workers are known to have measured damage thresholds as a function of pulse duration $[1,2,6,7]$. Consideration is limited to internal damage resulting from pulsed radiation.

\footnotetext{
${ }^{1}$ Figures in brackets indicate the literature references at the end of this paper. 2 Compare references 1 and 2
} 
Damage from laser radiation always occurs because sufficient energy has been absorbed to drive some kind of failure mechanism, but the ways in which laser energy can be absorbed and lead to damage are numerous. Some of them require imperfections in the material or depend on properties which are subject to control during the growing or melting process. Others are a result of more intrinsic properties and constitute fundamental limitations on the performance of the material.

\section{1 Absorption by Inclusions or Bulk Absorption}

Inclusions may be highly absorbing at the laser frequency. They can, therefore, cause several local heating effects leading to damage by thermal expansion, melting of the surrounding material, or other means $[6,8]$. For purposes of this analysis damage is assumed to result from the stress associated with thermal expansion.

During a very short pulse of duration $\Delta t$, or for a large inclusion of radius $R$ (such that $R^{2} / \Delta t>$ $D_{i}>D_{h}$, where $D_{i}$ and $D_{h}$ are the thermal diffusivities of the inclusion and host matrix respectively) the inclusion surface is heated to a depth of roughly $\left(D_{i} \Delta t\right)^{1 / 2}$, but very little heat is conducted into the surrounding material [9]. The laser energy density $\boldsymbol{d}_{\mathrm{d}}$ (energy per unit cross-sectional area) required to produce a damaging stress $S$ in the host at the surface of the inclusion due to expansion of the heated outer shell is approximately

$$
\oint_{d} \cong\left(C_{i} k_{h} D_{i}^{1 / 2} S / \beta_{i} e_{\lambda} M\right)(\Delta t)^{1 / 2}
$$

Here $C$ is the heat capacity per unit volume, $k$ is the volume compressibility, $\beta$ is the volume expansion coefficient, and $e_{\lambda}$ is the spectral emissivity of the inclusion at the laser wavelength. It is assumed that meaningful average values of these material properties can be used over the large temperature range involved and that heats of fusion and vaporization can be neglected to a first approximation. In addition, radiative heat transfer is taken as small compared to conductive transfer [8] in this and all following cases. The use of $M$ is necessary to correct for the modified effective cross-section of sub-wave length inclusions [10]. Its value is approximately unity for $\mathrm{R} \geq \lambda / 20$ and $20 \mathrm{R} / \lambda$ for $\mathrm{R}<\lambda / 20$.

Depending on the relative values of $D_{i}$ and $D_{h}$ there may be a range of pulse duration and inclusion size $\left(D_{i} \sim R^{2} / \Delta t>D_{h}\right)$ for which essentially the whole inclusion is heated during $\Delta t$ while conduction into the host matrix is still negligible. In this case the threshold for damage due to stress from expansion of the inclusion is given by

$$
\oint_{\mathrm{d}} \cong\left(4 \mathrm{C}_{\mathrm{i}} \mathrm{k}_{\mathrm{h}} \mathrm{S} / 3 \beta_{\mathrm{i}} \mathrm{e}_{\lambda} \mathrm{M}\right) \mathrm{R}
$$

Finally for long pulses or very small inclusions $\left(D_{i}>D_{h}>R^{2} / \Delta t\right)$ a considerable volume of the surrounding material is heated by conduction during $\Delta t$, and both the inclusion and the host matrix will undergo thermal expansion. In the absence of a significant difference in the expansion coefficients of the inclusion and host materials, attainment of a large temperature gradient in the host is now necessary to cause damage [11]. More specifically, the difference between the strain at some distance $r$ due to the heating of the host material in the volume (4/3) $\pi \mathrm{r}^{3}$ and the strain which would have occured at $r$ solely due to the local temperature change $\Delta \mathrm{T}$ must cause a stress equal to the breaking strength $S$ of the host. This requirement can be expressed by

$$
\mathrm{S}=\frac{\beta}{\mathrm{k}}\left(\Delta \mathrm{T}_{\mathrm{m}}-\Delta \mathrm{T}\right)
$$


where the mean temperature change $\Delta \mathrm{T}_{\mathrm{m}}$ is defined by

$$
\Delta \mathrm{T}_{\mathrm{m}} \equiv \frac{3}{\mathrm{r}^{3}} \int_{0}^{\mathrm{r}} \Delta \mathrm{T}(\mathrm{u}) \mathrm{u}^{2} \mathrm{du}
$$

Treating the inclusion as a continuous spherical surface source generating heat at a constant rate during the pulse [12], the minimum laser energy density required to cause damage is found to be on the order of

$$
\varepsilon_{\mathrm{d}} \approx\left(10 \mathrm{C}_{\mathrm{h}} \mathrm{k}_{\mathrm{h}} \mathrm{D}_{\mathrm{h}} \mathrm{S} / \beta_{\mathrm{h}} \mathrm{e}_{\lambda} \mathrm{M}\right)(\Delta \mathrm{t} / \mathrm{R})
$$

The derivation of eq. (5) ignores the fact that in this small inclusion limit the host may be heated to the extent that it can flow and become immune to fracture damage even at distances large compared to $R$. If such flow occurs in the short time of the laser pulse duration, then to cause fracturing the breaking strength $\mathrm{S}$ must be exceeded at the liquid-solid boundary, and this requires a higher laser energy density. Although this may be an important consideration for numerical calculations [13], it is not expected to change the dependence of eq. (5) on $\Delta t$ and $R$.

Equations (1), (2), and (5) give the dependence of damage threshold on inclusion radius and pulse duration for three limiting cases. Note that $\&_{d}$ has a minimum as a function of $R$. The pulse duration dependence is summarized in figure 1 and compared with experimental data for two different glasses, one which is known to contain platinum inclusions and one which is presumed to by virtue of its having been melted in a platinum crucible. If the inclusions are more accurately described as disks than as spheres, as is suspected in ruby [8], and if the face of the disk is illuminated rather than the edge, then the same $R$ and $\Delta t$ dependences are expected provided the $R$ in $M$ and in eq. (5) is taken as the disk's radius and the $\mathrm{R}$ in eq. (2) as its thickness.

Bulk absorption resulting, for example, from impurities or color centers can also cause laser damage either by simple heating to a temperature which permanently alters the optical properties of the material or by fracture of the region surrounding the beam by thermal expansion [9]. When thermal conduction out of an illuminated path of radius $r_{b}$ is negligible $\left(r_{b}^{2} / \Delta t>D\right)$, the laser energy densities required to cause a temperature change $\Delta T$ in the beam or a stress $S$ at its boundary are respectively,

$$
\varepsilon_{\mathrm{d}}=\mathrm{C} \Delta \mathrm{T} / \alpha
$$

and

$$
\varepsilon_{\mathrm{d}} \cong \mathrm{CkS} / \beta \alpha
$$

where $\alpha$ is the bulk absorption coefficient and the other symbols are as defined after eq. (1). When conduction is limited by the finite radial dimension $R_{S}$ of the sample instead of by thermal diffusivity, eq. (6) must be multiplied by $R_{s}^{2} / r_{b}$, and eq. (7) does not apply at all since there will be no unheated material to be strained.

When conduction becomes important $\left(\mathrm{r}_{\mathrm{b}}^{2} / \Delta \mathrm{t}<\mathrm{D}<\mathrm{R}_{\mathrm{s}}^{2} / \Delta \mathrm{t}\right)$, the illuminated region can be treated as a continuous line source [12] supplying heat to the surrounding volume. Then the energy densities required to cause a damaging temperature change $\Delta \mathrm{T}$ or a thermal stress $\mathrm{S}$ in the vicinity of the beam are respectively,

$$
\delta_{\mathrm{d}} \approx\left[4 \mathrm{CD} \Delta \mathrm{T} / \alpha \ln \left(4 \mathrm{D} \Delta \mathrm{t} / \mathrm{r}_{\mathrm{b}}^{2}\right)\right]\left[\Delta \mathrm{t} / \mathrm{r}_{\mathrm{b}}^{2}\right]
$$




$$
\S_{\mathrm{d}} \approx(4 \mathrm{CkDS} / \beta \alpha)\left(\Delta t / \mathrm{r}_{\mathrm{b}}^{2}\right)
$$

The derivation of eq. (9) follows the same arguments as those presented for eqs. (3) through (5).

It is possible, of course, that processes other than ordinary bulk absorption contribute to general heating of the irradiated volume at laser intensity levels. The absorption coefficient $\alpha$ should be understood to include all mechanisms for which the heat deposited is directly proportional to the local laser energy density. Experimental data at a pulse duration of one millisecond is available for glass intentionally doped with iron [9]. In figure 2 the dependence of the bulk absorption damage thres hold on $\Delta t$ and $r$ as given by eqs. (6) through (9) is fit to a data point for $\alpha=10^{-1} \mathrm{~cm}^{-1}$.

\section{2. Conduction Electron Absorption}

It may be possible for an electron in the conduction band of a material to be accelerated by the laser field to an energy greater than the band gap [1]. Each such electron would then be capable of exciting another electron into the conduction band by impact ionization, leading to an exponential increase in the number of conduction electrons $N$.

$$
N=N_{0} 2^{t / t^{\prime}}
$$

where $t^{\prime}$ is the time required to accelerate an electron to the energy of the conduction band. Interaction of the growing number of electrons with the lattice could cause electron avalanche breakdown.

Conduction electrons absorb photons from the laser field only in conjunction with impurity and phonon scattering. However, scattering also extracts energy from the electrons; so the first requirement which must be met to create an electron avalanche is that the electric field be strong enough to cause a net increase of electron energy in time until the band gap energy is reached. This requirement determines a minimum value of electric field $\mathrm{E}_{\mathrm{min}}$ and an associated laser power density $P_{d}$ (power per unit cross-sectional area) at which electron avalanche will occur [1, 14]. Since the phonon density decreases at lower temperatures, $P_{d}$ will increase with decreasing temperature as long as phonon scattering predominates. At low enough temperatures, however, $P_{d}$ will reach a temperature independent limit set by impurity scattering.

A second requirement for the electron avalanche to lead to actual breakdown is that the field be strong enough to cause the creation of a sufficient number of electrons during the pulse. Considering the problem classically and assuming collision losses to be negligible, the time derivative of electron energy can be written as $[1,15]$

$$
\mathrm{d} \xi e^{/ \mathrm{dt}}=\mathrm{f}\left(\dot{\varepsilon}_{\mathrm{e}}\right) \mathrm{E}_{\mathrm{o}}^{2}
$$

where $f\left(\varepsilon_{e}\right)$ is some function of $\&_{e}$. The time $t$ is then given by

$$
t^{\prime}=E_{0}^{-2} \int_{0}^{\xi} \mathrm{c}[f(\varepsilon)]^{-1} \mathrm{~d} \delta,
$$

where $\&$ is the energy needed to raise an electron to the conduction band. Substituting $t^{\prime}$ from eq. (12) into eq. (10) and solving for the energy density required to produce $\mathrm{N}_{\mathrm{b}}$ conduction electrons in a time $\Delta t$ gives

$$
\varepsilon_{\mathrm{d}} \propto \mathrm{E}_{\mathrm{o}}^{2} \Delta t \propto \ln \left(\mathrm{N}_{\mathrm{b}} / \mathrm{N}_{\mathrm{o}}\right)
$$


Since both requirements noted above must be met, and provided that the necessary number of electrons $\mathrm{N}_{\mathrm{b}}$ is independent of $\Delta \mathrm{t}$, the dependence of $\delta_{\mathrm{d}}$ on pulse duration changes from linear with $\Delta t$ for long pulses to independent of $\Delta t$ for short pulses.

In the preceding paragraphs it has been tacitly assumed that the lifetime of electrons in the conduction band $\tau_{\mathrm{e}}$ is long compared to $t^{\prime}$, the time between generations in the electron avalanche. If $\tau$ e $/ t^{\prime}$ is less than about five, however, the avalanche process is slowed and a somewhat greater energy density is required to cause damage at a given pulse duration. When $\tau_{\mathrm{e}} / \mathrm{t}^{\prime}$ is less than two, avalanche development is impossible. Taking this into account and noting the variation of $t^{\prime}$ with $E_{\text {。 }}$ as given by eq. (12), one concludes that at most the effect of a short $\tau_{\mathrm{e}}$ is to make the transition between the two forms of $\Delta t$ dependence more gradual and to shift the constant power threshold for long, pulses from the one determined by $E_{\text {min }}$ to a higher nearly constant power required to keep $\tau_{\mathrm{e}} / \mathrm{t}^{\prime}$ greater than two. A complicating factor which has not been treated here is the possibility of raising electrons to intermediate states short of the conduction band [16].

Some difference of opinion exists over whether electron avalanche breakdown actually occurs. Recent calculations [17] of energy transfer rates from an optical field to a conduction electron and from an electron to the lattice via longitudinal phonon scattering, suggest that an electron avalanche is unlikely in ruby or sapphire because the electron energy rises only slightly in transfering energy from the optical field to the lattice. These calculations were made to lowest order in optical intensity and electron-phonon coupling, and it is possible that higher order solutions will lead to different conclusions. In the meantime, however, the effects of low energy conduction electrons should be considered.

The transfer of energy from a field $\mathrm{E}$ with wavelength $\lambda$ to electrons of effective mass $\mathrm{m} *$ coupled to longitudinal optical phonons may be characterized by an optical absorption coefficient [17]

$$
\alpha=\left(16 \pi^{2} \mathrm{Ne}^{2} \gamma\right) /\left(\lambda \mathrm{m} * \mathrm{E}_{0}^{2}\right),
$$

where $\mathrm{N}$ is the conduction electron density and $\gamma$, the rate at which the field does work on an electron, is proportional to $E_{O}^{2}$. One possibility is that a substantial number of electrons are excited early in the laser pulse from impurity levels close to the conduction band. If $\mathrm{N}$ remains roughly constant for the rest of the pulse, then these electrons simply contribute to the bulk absorption described in eqs. (6) through (9).

At the high intensity levels which can be reached in lasers, conduction electrons may also be created by the simultaneous absorption of several photons [18,19]. Various schemes have been proposed for two-, three-, and four-photon absorption in ruby [15, 19-23] and four-photon absorption (of ruby radiation) in glass [24]. Since the probability for $n$-photon absorption increases with decreasing $n$, anything which affects the number of photons required to get electrons into the conduction band will influence the probability of the multiphoton process. Clearly the photon frequency is an important parameter. In addition, elevating electrons to excited states by optical pumping or altering the energy level structure by addition of impurities [4] may affect the importance of multiphoton absorption.

The probability of $\mathrm{n}$-photon ionization depends on the electric field strength through a factor $\mathrm{E}^{2 \mathrm{n}}$, so $\mathrm{N} \propto \mathrm{E}^{2 \mathrm{n}} \Delta \mathrm{t}$. If $\alpha$ of eq. (14) is averaged over the pulse duration $\Delta \mathrm{t}$ and substituted in either eq. (6) or eq. (7), the dependence of the damage threshold on pulse duration is given by

$$
\delta_{d} \propto \Delta t^{(n-1) /(n+1)},
$$

subject to the condition $\mathrm{r}_{\mathrm{b}}^{2} / \Delta \mathrm{t} \gg \mathrm{D}$ as noted before eq. (6).

In figure 3 the pulse duration dependence predicted for the electron avalanche breakdown threshold is fit to a group of experimental points thought to represent avalanche damage [1]. A curve for damage by low energy conduction electrons created through multiphoton absorption is also given, although no related data is available. 


\section{3. Stimulated Brillouin Scattering}

Under certain conditions it is possible to combine the electrostrictive generation of an acoustic wave by the interaction of two electromagnetic waves (EMW) with the generation of an EMW by the Bragg diffraction of an initial EMW from an acoustic wave. This leads to stimulated Brillouin scattering (SBS), an internal feedback mechanism for amplifying an acoustic wave ( $\omega_{a}$, $\underset{\sim}{\mathrm{k}}$ ) and a secondary EMW $\left(\omega_{S},{\underset{\sim}{s}}_{s}\right)$ at the expense of an input laser beam $\left(\omega_{\ell},{\underset{\sim}{k} \ell}\right)$ [25]. At high enough power levels damage may occur from the mechanical stresses associated with the acoustic wave [26]. The stress arising from heating due to damping of the acoustic wave is negligible by comparison [27].

Depending on the pulse duration, transient or steady state SBS theories may be appropriate [28, 29]. In either case the acoustic power level reached by the end of the pulse will depend on the length of the interaction region, the mechanical parameters of the material (such as acoustic attenuation and elastic constants), the electromagnetic parameters (such as permeability, permitivity, and index of refraction), and the Pockel's elasto-optic constants which couple mechanical and optical phenomena in the material. In addition, the pulse duration $\Delta t$ is of primary importance since a finite time, dependent on the laser power density $P_{d} l$, is required to generate an acoustic power $P_{d a}$. Experimental evidence of this delay in acoustic generation has been found in quartz [27], glass [30], and $\mathrm{CS}_{2}[31]$.

For pulses too short to establish steady state SBS and in the limit of an infinite interaction length [28]

$$
\mathrm{P}_{\mathrm{da}} \propto \Delta t \mathrm{P}_{\mathrm{ds}}
$$

and $[28,32]$

$$
\mathrm{P}_{\mathrm{ds}} \propto \exp \left[\left(\mathrm{AP}{ }_{\mathrm{d} \ell} \Delta \mathrm{t}\right)^{1 / 2}\right]=\exp \left(\mathrm{A}^{1 / 2} \varepsilon_{\mathrm{d} \ell}^{1 / 2}\right) .
$$

Here $\mathrm{A}$ is determined by material parameters. Combining eqs. (16) and (17) leads to

$$
\&_{\mathrm{d} l} \propto\left[\ln \left(\frac{\mathrm{BP} \mathrm{da}}{\Delta t}\right)\right]^{2}
$$

where $B$ depends on the material and the laser frequency. Since the breaking strength of the material corresponds to a particular value of $\mathrm{P}_{\mathrm{da}}$, the laser energy density required for damage will increase as $\Delta t$ is reduced

The use of eq. (17) assumes that $\mathrm{P}_{\mathrm{ds}}$ does not reach a limiting value in the time $\Delta \mathrm{t}$. There may be an intermediate range of $\Delta$ t for which $\mathrm{P}_{\mathrm{ds}}$ does reach its limiting value but for which eq. (16) is still correct [28]. In that case

$$
\mathrm{P}_{\mathrm{da}} \propto \Delta t \mathrm{P}_{\mathrm{d} \ell}=\ell_{\mathrm{d} \ell}
$$

so that the laser energy density required for damage is independent of $\Delta t$. Finally, for long enough $\Delta t$, steady state SBS can be achieved. Then $P_{\text {ds }}$ increases exponentially with $P_{d \ell}[29,32]$ (until $P_{d s}$ approaches $\left.P_{d} \ell\right)$ and $P_{d a}$ is proportional to the product of $P_{d s}$ and $P_{d} \ell[27]$. Thus for long $\Delta t$, damage occurs at a constant power level and $\varepsilon_{\mathrm{d} \ell} \propto \Delta \mathrm{t}$. The $\Delta$ t dependence of the threshold for damage by SBS is illustrated in figure 4. 
For a non-lasing sample irradiated by collimated laser light, the peak SBS acoustic intensity occurs at the entrance end of the sample [33], suggesting that damage caused by SBS should be found very near the entrance face. That the damage is not found at this location in a particular experiment does not provide sufficient basis for eliminating SBS as a possible damage mechanism, however, since a focused probe beam or the self-focusing mechanisms described in the next section may cause the peak acoustic intensity to be generated further from the surface

\section{4. Self-Focusing}

All of the mechanisms discussed to this point are more likely to lead to damage for a given $\Delta t$ if the laser energy density is increased. Thus any degree of self-focusing, i. e. any reduction of the laser beam diameter below the value predicted from the optical properties of the unirradiated material, is potentially important in the creation of laser damage. Such a reduction can result from any process which leads to an increase of the index of refraction with increasing light intensity. In solids, where gross molecular motion is frozen out, possible self-focusing mechanisms include electrostriction [3, 34], electronic distortion [35-37] or molecular librations [38], and absorptive heating [39].

Electrostriction is a likely mechanism for self-focusing in transparent dielectrice under Qswitched and long pulse laser ir radiation [3]. The net electrostrictive force at any point in a material is proportional to the gradient of the square of the electric field. Thus a radially symmetric beam whose intensity decreases smoothly with distance from the axis ${ }^{3}$ causes a radially symmetric compression and an associated change in the refractive index which leads to self-focusing. Since the process is a dynamic one, the compression takes the form of a radially propagating acoustic wave with velocity $v_{a}$. For a given initial beam radius $r_{b}$, a minimum time $\tau=r_{b} / v_{a}$ is required for the acoustic wave to propagate across the beam. In a single pass through a lossless medium and for $\Delta t \gg \tau$, self-focusing will overcome normal diffraction spreading from a collimated beam at the self-trapping threshold given by

$$
\varepsilon_{d}=C_{1} g\left(n_{0}\right) \lambda^{2} \Delta t / r_{b}^{2}
$$

where $C_{1}$ depends on the elastic properties of the material, $g$ is a function of the original refractive index $n_{0}$, and $\lambda$ is the laser wavelength in vacuum.

For $\Delta t \ll \tau$ the acoustic wave does not have time to develop fully, and trapping requires an increased energy density given by

$$
\varepsilon_{\mathrm{d}}=\mathrm{C}_{2} g\left(\mathrm{n}_{\mathrm{o}}\right) \lambda^{2} / \Delta \mathrm{t}
$$

Equations (20) and (21) predict a constant total power threshold [34] for $\Delta t>\tau$ and an energy density threshold varying inversely with $\Delta t$ for $\Delta t<\tau$. To illustrate this behavior, curves based on calculations for ruby laser radiation in BK-7 glass [40] are plotted as solid lines in figure 5 along with a data point for damage accompanied by self-focusing from a $40 \mu$ initial beam radius [41]. The exact agreement between this measurement and the calculations is probably fortuitous since the damage level should exceed the trapping threshold in order to reduce the beam cross-section significantly.

The beam must also travel a finite distance in the material for self-focusing to have a notable effect. An approximate focusing length $I_{f}$ can be defined by equating the optical path length (n + $\Delta n) L_{f}$ along the beam axis with the path length $n_{0}\left(L_{f}^{2}+r_{b}^{2}\right)^{1 / 2}$ from the beam perimeter to the axis, where $\Delta \mathrm{n}$ is the change in refractive index on axis [42]. Using appropriate limiting forms for $\Delta \mathrm{n}$ [40] and requiring $L_{f}$ to equal the sample length $L$, gives

\footnotetext{
3 Large local intensity variations over the beam cross-section can cause localized self-focusing resulting in the formation of multiple filaments
} 


$$
\varepsilon_{d} \propto r_{b}^{2} \Delta t / L^{2}
$$

for $\Delta t \gg \tau$ and

$$
\varepsilon_{d} \propto r_{b}^{4} /\left(L^{2} \Delta t\right)
$$

for $\Delta t<\tau$. When a sample is irradiated by a sharply focused beam, the length which is useful for development of self-focusing is less than L. In the extreme case the sample length loses all significance because the energy density is high only over a short distance near the focused minimum.

The dashed electrostriction curves in figure 5 have been located on the energy density axis by fitting eq. (22) to damage measurements [41] made in BK-7 with a $125 \mu$ beam. At about this beam size the damage threshold was observed to begin rising with increasing beam radius. Since significant focusing will not occur unless the requirements of both eqs. (20 and (21) and those of eq. (22) and (23) are met, the threshold for damage initiated by electrostrictive self focusing has a minimum as a function of both $r$ and $\Delta t$. It should be emphasized that the increase in trapping threshold for short pulses does not occur because of a reduced acoustic response, but rather because of the time $\left(\tau=\mathrm{r}_{\mathrm{b}} / \mathrm{v}_{\mathrm{a}}\right)$ required for that response to fully develop. In other words, even extremely short pulses can be focused by this mechanism if they are preceded by other pulses which initiate acoustic lens formation. A mode-locked laser produces just such a pulse train.

Other self-focusing processes with much shorter relaxation times contribute a quadratic term to the refractive index $n$, even for a single pulse with duration on the order of picoseconds. These include electronic distortion [35-37] and molecular libration [38]. Setting the approximate focusing length $L_{f}$ as defined before eq. (22) equal to the Raleigh range for a beam of initial radius $r_{b}$, gives an order of magnitude result for the trapping threshold

$$
\varepsilon_{\mathrm{d}} \approx\left(\lambda^{2} \mathrm{C} / 16 \pi^{3} \mathrm{n}_{2}\right)\left(\Delta \mathrm{t} / \mathrm{r}_{\mathrm{b}}^{2}\right)
$$

where $n=n_{0}+n_{2} E_{0}^{2}$. Also, following the same arguments for the effect of sample length as were given for electrostrictive focusing leads to the further requirement,

$$
\varepsilon_{\mathrm{d}} \approx\left(\mathrm{cn} \mathrm{n}_{\mathrm{o}}^{2} / 16 \pi \mathrm{n}_{2}\right)\left(\mathrm{r}_{\mathrm{b}}^{2} \Delta \mathrm{t} / \mathrm{L}^{2}\right)
$$

Using $n_{2}=2 \times 10^{-13}$ esu ${ }^{4}[36]$, plots of eqs. (24) and (25) for ruby radiation in glass have been added to figure 5 for representative values of beam radius. Again the trapping threshold is indicated by solid lines and the energy requirement resulting from a finite (10 cm) sample length by dashed curves. It is clear that the electronic distortion or molecular libration mechanisms dominate for very short pulses, while electrostriction becomes competitive for Q-switched or longer duration pulses. In any case the energy density required for significant focusing has a minimum as a function of initial beam radius, and for mechanisms with relaxation times shorter than the pulse duration the location of this minimum is found by equating eqs. (24) and (25). The result is

$$
\mathrm{r}_{\min } \approx\left(\lambda \mathrm{L} / \mathrm{n}_{\mathrm{o}} \pi\right)^{1 / 2}
$$

independent of $\mathrm{n}_{2}$ and $\Delta \mathrm{t}$.

${ }^{4}$ This is approximately the value measured for $1.06 \mu$ radiation in fused silica. 
When $\mathrm{dn} / \mathrm{d} \mathrm{T}$ is positive, heating by absorption at the laser frequency will also contribute to selffocusing. Even materials whose total derivative is found to be negative by a steady state measurement, can exhibit thermal self-focusing for pulses sufficiently short $(\Delta t<<\tau)$ that the effective value of $\mathrm{dn} / \mathrm{d} \mathrm{T}$ is just the positive partial derivative at constant density [39]. Moreover, since unheated material surrounding a laser irradiated area will limit thermally induced density changes, it may be possible to obtain thermal self-focusing for longer pulses as well [43].

The approximate threshold for significant thermal self-focusing is given by the larger of eqs. (24) and (25) with $\mathrm{n}_{2}$ taken as

$$
\mathrm{n}_{2}=(\mathrm{dn} / \mathrm{dT})_{\text {eff }}(\alpha \mathrm{cn} \Delta \mathrm{t} / 8 \pi \mathrm{C})
$$

provided only that $\Delta t \ll r_{b}^{2} / D$ so that the heat lost from the beam path during the pulse is negligible. As with the other mechanisms the threshold has a minimum as a function of initial beam radius, but thermal self-focusing is unique in that no pulse duration dependence is expected [42] provided $\Delta t$ is much less than both $\tau$ and $\mathrm{r}_{\mathrm{b}}^{2} / \mathrm{D}$. Some tentative experimental evidence exists to confirm such a lack of $\Delta$ tependence for self-focusing of short pulses [7].

The importance of this mechanism relative to those plotted in figure 5 can be judged by comparing values of $\mathrm{n}_{2}$. For $(\partial \mathrm{n} / \partial \mathrm{T})_{\rho}=5 \times 10^{-6}[44], \alpha=0.25 \% \mathrm{~cm}^{-1}, \Delta \mathrm{t}=10^{-7} \mathrm{sec}$, and $\mathrm{C}=0.9 \mathrm{cal} /$ $\mathrm{cm}^{3}-{ }^{\circ} \mathrm{K}$, the $\mathrm{n}_{2}$ for thermal self-focusing is approximately $0.6 \times 10^{-13}$ esu. This is somewhat less than the $\mathrm{n}_{2}$ previously noted for an electronic distortion type of focusing. However, a rather small increase in absorption would make the two comparable at this pulse duration. So, although for very short pulses thermal self-focusing is expected to be negligible compared to other mechanisms, it must be regarded as potentially important when $\Delta$ t approaches 100 nanoseconds or when absorption at the laser frequency is high.

Thermal effects also occur due to other radiation sources such as flash lamps [45]. These are not considered here.

\section{Conclusions}

Pulse duration is an important parameter for each of the mechanisms discussed in the preceding pages. As shown in the equations of Section 2 and in figures 1 through 5 , the form of the time dependence varies from one mechanism to another. This raises the possibility that for a given material different mechanisms are responsible for initiating damage in different ranges of pulse duration and suggests that experimental identification of damage mechanisms can be greatly aided by measuring the pulse duration dependence of the damaging energy density. The incident beam radius has also been demonstrated to be an important parameter, particularly for self-focusing mechanisms, which also are effected by sample length. Since most mechanisms are expected to cause damage at energy densities independent of beam radius and sample length, variation of these parameters can be useful both in determining whether self-focusing is involved in a particular laser damage problem and in designing systems less susceptible to such damage.

Having explicitly shown the importance of the above parameters and alluded to the significance of others such as temperature, optical pumping conditions, beam focusing, and lase $r$ frequency, it is appropriate to emphasize the need to carefully control and specify these parameters in any damage experiment. Hopefully such experiments combined with further theoretical efforts will lead to unambiguous identification of limiting damage mechanisms over the useful range of laser system parameters. Progress in preventing damage can then be expected through discrimination against the mechanisms involved.

\section{Acknowledgements}

Discussions with C. M. Stickley, R. A. Bradbury, E. L. Kerr, C.R. Giuliano, R. W. Hellwarth, and many others with whom I have enjoyed less extensive contact are gratefully acknowledged. 
Subscripts

$$
\begin{aligned}
& a=\text { acoustic } \\
& \mathrm{h}=\text { host } \\
& \mathrm{i}=\text { inclusion } \\
& 1=\text { laser } \\
& \mathrm{s}=\text { secondary }
\end{aligned}
$$

Symbols

$\alpha=$ bulk absorption coefficient

$\beta=$ thermal volume expansion coefficient

$\mathrm{C}=$ heat capacity per unit volume

$c=$ velocity of light in vacuum

$\mathrm{D}=$ thermal diffusivity

$E=$ instantaneous electric field strength

$E_{\mathrm{O}}=$ electric field amplitude

$\mathrm{e}=$ charge on an electron

$\mathrm{e}_{\lambda}=$ spectral emissivity at the laser wavelength

$\hat{\delta}_{c}^{\lambda}=$ band gap energy

$\varepsilon_{\mathrm{d}}=$ energy density (energy per unit cross-sectional area)

$\delta_{\mathrm{e}}=$ electron energy

$\mathrm{k}=$ volume compressibility

$\mathrm{k}=$ wave vector

$\widetilde{\mathrm{L}}=$ sample length

$L_{\mathrm{f}}=$ focusing length

$\lambda=$ wavelength in vacuum

$M=$ multiplication factor to correct particle cross-section for Mie effect

$m *$ = electron effective mass

$N=$ conduction electron number density

$\mathrm{N}_{\mathrm{b}}$ = value of $\mathrm{N}$ required for breakdown

$\mathrm{n}=$ number of photons absorbed in $\mathrm{n}$-photon ionization

$\mathrm{n}=$ refractive inde $\mathrm{x}$

$\mathrm{n}_{\mathrm{o}}=$ original refractive index

$\mathrm{n}_{2}=$ coefficient for quadratic term in expression for $\mathrm{n}$

$\Delta \mathrm{n}=$ change in $\mathrm{n}$

$\mathrm{P}_{\mathrm{d}}=$ power density (power per unit cross-sectional area)

$\mathrm{R}=$ inclusion radius

$\mathrm{R}_{\mathrm{S}}=$ sample radius

$\mathrm{r}=$ distance from center of inclusion

$\mathrm{r}_{\mathrm{b}}=$ beam radius

$\mathrm{S}=$ breaking strength, damaging stress

$\mathrm{T}=$ temperature

$\mathrm{t}=\mathrm{time}$

$t^{\prime}=$ time required to accelerate an electron to the energy of the conduction band

$\Delta \mathrm{t}=$ pulse duration

$\tau=$ time required for acoustic wave to propagate across the beam

$\tau_{\mathrm{e}}=$ conduction electron lifetime

$v=$ velocity

$\omega=$ angular velocity (frequency times $2 \pi$ )

\section{References}

[1] G. M. Zverev, T. N. Mikhailova, V. A. Pashkov, and N. M. Soloveva, Soviet Phys. JETP 26, 1053 (1968).

[2] P. V. Avizonis and T. Farrington, Appl. Phys. Letters 7, 205 (1965).

[3] E. L. Kerr, ONR Contract N00014-67-C-0468,
Final Report (1968)

[4] G. Nath and G. Walda, Z. Naturforsch. $23 a, 624$ (1968).

[5] J. Davit, J. Appl. Phys. 39, 6052 (1968). 
[6] C. G. Young and R. F. Woodcock in Damage in Laser Glass ASTM STP 469, ed. A. J.

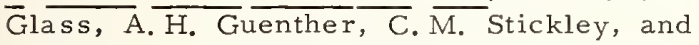
J.D. Myers (American Society for Testing and Materials, Philadelphia, 1969).

[7] J. Davit in Damage in Laser Glass ASTM STP 469, ed A.J. Glass, A. H. Guenther, C.M. Stickley, and J.D. Myers (American Society for Testing and Materials, Philadelphia, 1969).

[8] F.R. Charvat, G. A. Keig, O.H. Nestor, P. E. Otten, and J.C. Smith, Government Contract NONR-4132(00), Annual Technical Summary Report (1967).

[9] R. D. Maurer, W. W. Lester, and M. E. Vance, Government Contract N00014-66C0159, Final Technical Report (1967).

[10] M. Born and E Wolf, Principles of Optics (Pergamon Press, Oxford, 1965), 3rd Revised ed. , p663.

[11] L. M. K. Boelter, V. H. Cherry, H. A. Johnson, and R. C. Martinelli, Heat Transfer Notes (McGraw-Hill Book Co. , N. Y., 1965), p6 30

[12] H. S. Carslaw and J. C. Jaeger, Conduction of Heat in Solids (Oxford University Press, London, 1959), 2nd edition.

[13] R. W. Hopper and D. R. Uhlmann, submitted to Journal of Applied Physics.

[14] A. Wasserman, Appl. Phys. Letters 10, 132 (1967).

[15] E. A. Sviridenkov, Soviet Phys. - Solid State 9, 1917 (1968).

[16] Y. P. Raizer, Soviet Phys. - Usp. 8, 650 (1966).

[17] C. R. Giuliano, R. W. Hellwarth, L. D. Hess, and G. R. Rickel, Government Contract F19628-69-C-0277, Semi-annual Report No. 1 (1970).

[18] L. V. Keldysh, Soviet Phys. - JETP 20, 1307 (1965).

[19] F. V. Bunkin and A. M. Prokhorov, Soviet Phys. - JETP 21, 725 (1965).

[20] V. A. Pashkov and G. M. Zverev, Soviet Phys. - JETP 24, 516 (1967).

[21] T. P. Belikova, A. N. Savchenko, and E. A. Sviridenkov, Soviet Phys. - JETP 27, 19 (1968).

[22] T. P. Belikova and E. A. Sviridenkov, JETP Letters 3, 257 (1966).
[23] T.P. Belikova and E. A. Sviridenkov, JETP Letters 1, 171 (1965).

[24] B.S. Sharma and K. E. Rieckhoff, Can. J. Phys. 45, 3781 (1967).

[25] R. Y. Chiao, E. Garmire, and C. H. Townes, Proc. of the Enrico Fermi Int'l School of Physics, Course XXXI, ed. P.A. Miles (Academic Press, N.Y., 1964).

[26] R. Y. Chiao, C. H. Townes, and B.P. Stoicheff, Phys. Rev. Letters 12, 592 (1964).

[27] C. L. Tang, AF Contract AF19(628)-5677, Final Report (1968).

[28] N. M. Kroll, J. Appl. Phys. 36, 34 (1965).

[29] J. Walder and C. L. Tang, Phys. Rev. 155, 318 (1967).

[30] L. D. Khazov and A. N. Shestov, Optics and Spectroscopy XXV, 248 (1968).

[31] D. von der Linde, M. Maier, and W. Kaiser, Phys. Rev. 178, 11 (1969).

[32] E. F. Hagenlocker, R. W. Minck, and W. G. Rado, Phys. Rev. 154, 226 (1967)

[33] J. Walder and C. L. Tang, Phys. Rev. Letters 19, 623 (1967).

[34] R. Y. Chiao, E. Garmine, and C.H. Townes, Phys. Rev. Letters 13, 479 (1964).

[35] R. G. Brewer and C.H. Lee, Phys. Rev. Letters 21, 267 (1968).

[36] M. A. Duguay and J.W. Hansen, Spring Meeting of the Optical Society of Am. (1970).

[37] R. R. Alfano and S. L. Shapiro, Phys. Rev. Letters 24,592 (1970).

[38] R. Polloni, C. A. Sacchi, and O. Svelto, Phys. Rev. Letters 23, 690 (1969).

[39] R. L. Carman, A. Mooradian, P. L. Kelley, and A. Tufts, Appl. Phys. Letters 14, 136 (1969).

[40] E. L. Kerr, to be published in IEEE J. of Quantum Electronics (August 1970).

[41] .G. N. Steinberg and E. L. Kerr, unpublished.

[42] F.W. Quelle in Damage in Laser Glass ASTM STP 469, ed A.J. Glass, A. Guenther, C.M. Stickley, and J.D. Myers (American Society for Testing and Materials, Philadelphia, 1969).

[43] G. M. Zverev, E. A. Levchuk, E. K. Maldutis, and V.A. Pashkov, JETP Letters 11 , 108 (1970).

[44] E. Snitzer, private communication (June 1970). 
[45] R. L. Townsend, C. M. Stickley, and A. D. Maio, Appl. Phys, Letters 7, 94 (1965).

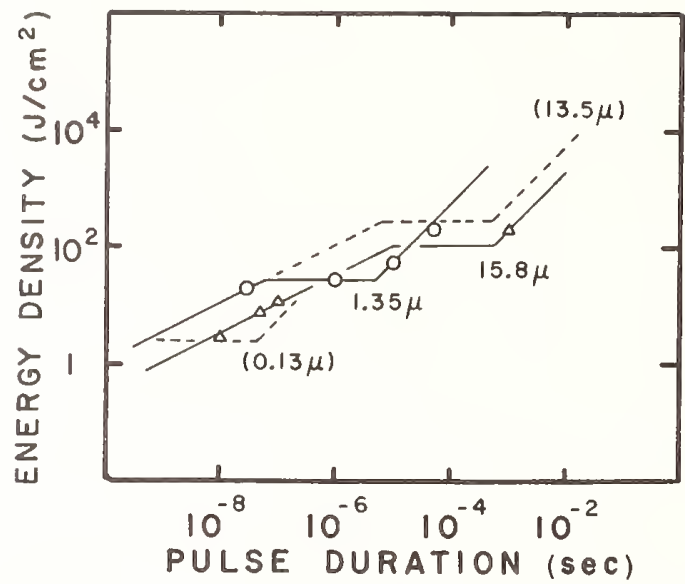

Fig. 1 Threshold for damage due to absorption by platinum inclusions in glass. The pulse duration and inclusion radius dependence of eqs. (1), (2), and (5) have been fit to data from reference [2] (indicated by $\Delta$ ) for glass known to contain Pt inclusions and data from reference [6] (indicated by $O$ ) for glass presumed to contain Pt inclusions. The inclusion radius $R$ and a scale factor for energy density were treated as free parameters and varied to obtain the best fit while requiring the break points in the curves to be at pulse durations of $R^{2} / D_{P t}$ and $R^{2} / D_{\text {glass }}$. The resulting inclusion sizes are indicated. The dashed curves and $R$ values in parentheses illustrate the theoretical dependence on $\mathrm{R}$ for the glass in reference [6].

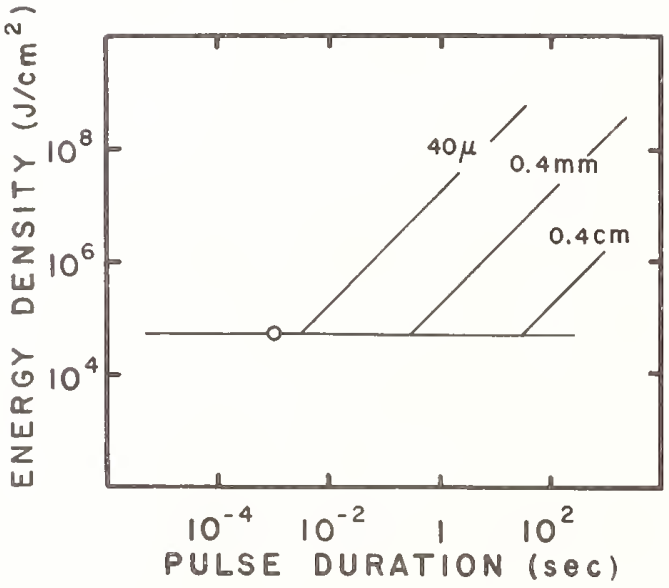

Fig. 2 Threshold for damage by bulk absorption for three beam radii in glass with an absorption coefficient of 0.1 $\mathrm{cm}^{-1}$. The pulse duration dependence of eqs. (6) through (9) is fit to data from reference [9] taken with millisecond pulses. The break points occur at $\Delta t=\mathrm{r}_{\mathrm{b}}^{2} / \mathrm{D}_{\text {glass, }}$, and the logarithmic dependence on $\Delta t / r_{b}^{2}$ in eq. (8) is neglected.

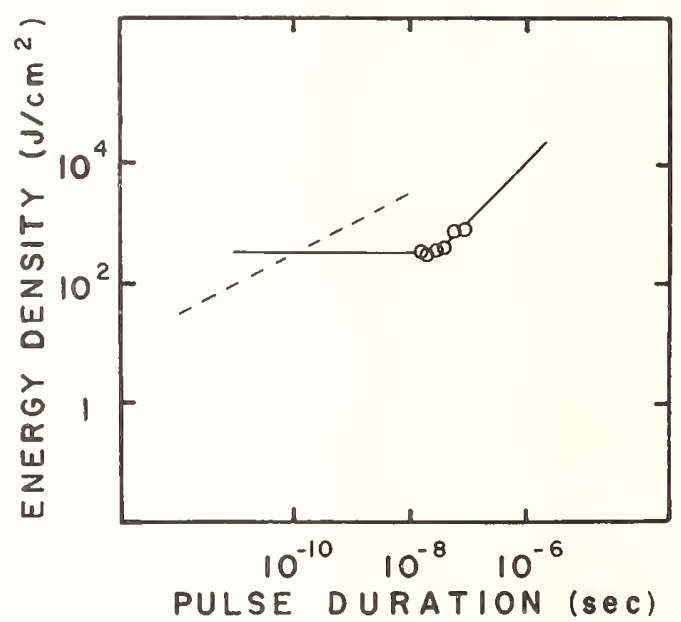

Fig. 3 Threshold for damage due to electron avalanche breakdown in ruby (solid line) and to low energy conduction electrons created by three photon ionization (dashed line). The constant power density requirement noted after eq. (10) and the constant energy density requirement of eq. (13) are fit to data from reference [1] for electron avalanche damage. The position of the dashed curve illustrating eq. (15) has been chosen arbitrarily since no data are a vailable. 


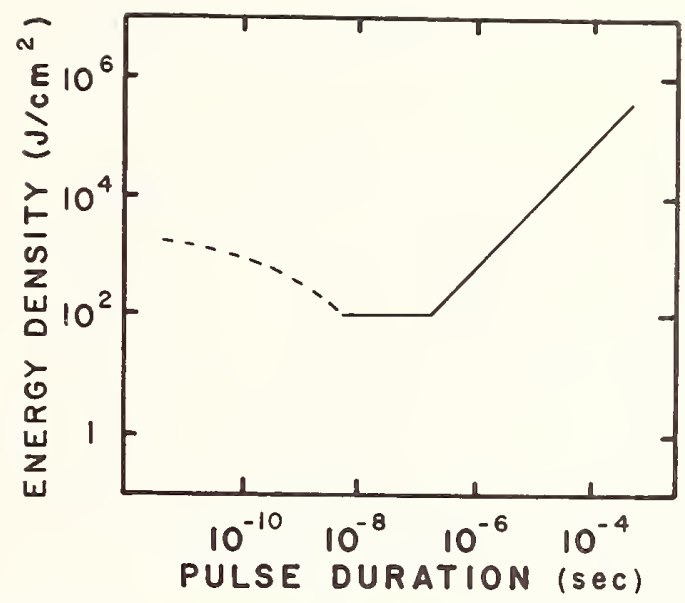

Fig. 4 Predicted pulse duration dependence of the threshold for damage due to stimulated Brillouin scattering. The energy scale is estimated from quartz and glass data in A. I. Ritus and A. A. Manenkov, JETP Letters 6, 349 (1967) and from quartz calculations in reference [26]. The time scale is estimated from quartz data in reference [29]. The exact form of the dashed part of the curve cannot be determined without knowing $B$ in eq. (18).

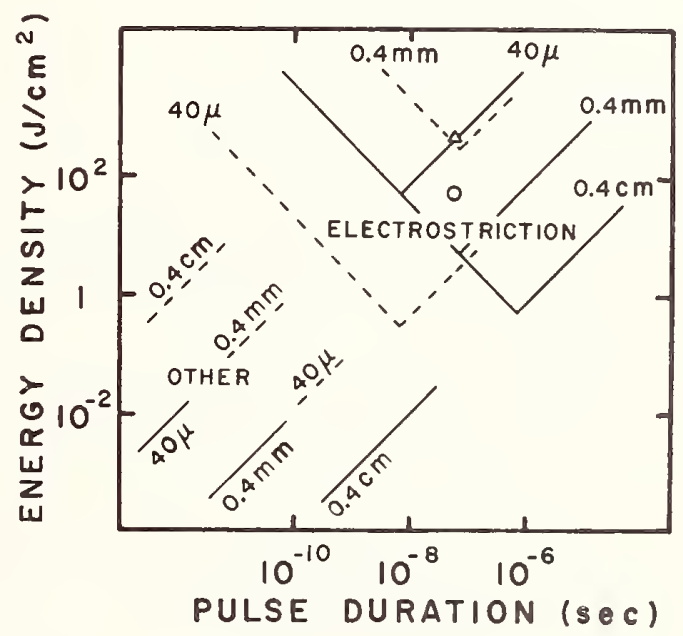

Fig. 5 Self-trapping of ruby radiation in glass for several beam radii by electrostriction and by mechanisms with a refractive index change which is quadratic in the electric field. The solid electrostriction curves, which indicate the trapping threshold, result from fitting eq. (20) to calculations in reference [40] and are compared with a data point ( $\Delta)$ from reference [41] for a $40 u$ beam. The dashed electrostriction curves, which indicate the additional requirement imposed by a $10 \mathrm{~cm}$ sample length, result from a fit of eq. (22) to a damage measurement in reference [41] with a $125 \mu$ beam (O). The other solid curves are a plot of the trapping threshold predicted by eq. (24) for $\mathrm{n}_{2}=2 \times 10^{-13}$ esu. while the other dashed curves are a plot of the sample length restriction given by eq. (25) for a $10 \mathrm{~cm}$ sample. 
Participants 1

E. D. Baird, Lawrence Radiation Lab., Livermore, California 94550

M. Bass, Raytheon Co., Waltham, Massachusetts 02154

R. W. Beck, Owens-Illinois Inc., Toledo, Ohio 43607

H. Bennett, National Bureau of Standards, Washington, D.C. 20234

R. J. Bieringer, Owens-Illinois Inc., Toledo, Ohio 43607

E. S. B1iss, A.F. Cambridge Research Lab., Bedford, Massachusetts 01730

N. L. Boling, Owens-Illinois Inc., Toledo, Ohio 43607

J. Davit, Campagnie Generale D'Electricite, 92-Marcousses, France

M. A. Duguay, Bel1 Telephone Labs, Murray Hil1, New Jersey 07974

D. F. Edwards, Colorado State Univ., Fort Collins, Colorado 80521

S. J. Ekstrom, Lawrence Radiation Lab., Livermore, California 94550

A. Feldman, Nationa1 Bureau of Standards, Washington, D.C. 20234

A. D. French, General Electric, Syracuse, New York 13201

D. H. Gill, Los Alamos Scientificlab., Los Alamos, New Mexico 87544

C. R. Giuliano, Hughes Research Labs, Malibu, California 90265

A. J. Glass, Wayne State Univ., Detroit, Michigan 48202

A. H. Guenther, Kirtland A.F.B., New Mexico 87117

R. Hellwarth, Hughes Research Labs, Malibu, California 90265

L. T. James, Lawrence Radiation Lab, Livermore, California 94550

P. M. Johnson, Lawrence Radiation Lab., Livermore, California 94550

C.R. Jones, Kirtland A.F.B., New Mexico 87117

Y. Ksander, Institute for Defense Analyses, Arlington, Virginia 20022

H. Lee, Owens-Illinois Inc., Toledo, Ohio 43607

J.R. Lenhoff, A.F. Cambridge Research Lab., Bedford, Massachusetts 01730

P. Mace, Los Alamos Scientific Lab., Los Alamos, New Mexico 87544

J. D. Myers, Owens-Illinois Inc., Toledo, Ohio 43607

C.A. Pitha, A.F. Cambridge Research Lab., Bedford, Massachusetts 01730

R. C. Puttbach, Litton Industries, Morris Plains, New Jersey 07950

L. R. Rothrock, Union Carbide Crystals, San Diego, California 92123

R. R. Rudder, Kirtland A.F.B., New Mexico 87117

P. M. Rushworth, Martin Marietta Corp., Orlando, Florida 32805

\footnotetext{
IApproximately 100 others who did not formally register, attended selected sessions.
} 
T. Saito, Kirtland. A.F.B., New Mexico 87117

C. Y. She, Colorado State Univ., Fort Collins, Colorado 80521

L. D. Smith, Harshaw Chemical Co., Solon, Ohio 44139

E. Snitzer, American Optical Co., Southbridge, Massachusetts 01550

J. B. Sonderman, Lawrence Radiation Lab., Livermore, California 94550

K. Steinbruegge, Westinghouse, Pittsburgh, Pennsylvania 15235

C. M. Stickley, A.F. Cambridge Research Lab., Bedford, Massachusetts 01730

C. C. Wang, Ford Scientific Lab., Dearborn, Michigan 48121

E. J. Woodbury, Hughes Aircraft Co., Culver City, California 90230

R. F. Woodcock, American Optica1 Corp., Sorthbridge, Massachusetts 01550

A. W. Young, Rank Precision Industries, Inc., Des Plaines, Illinois 60018

C. G. Young, American Optica1 Corp., Southbridge, Massachusetts 01550 

Latest developments in the subject area of this publication, as well as in other areas where the National Bureau of Standards is active, are reported in the NBS Technical News Bulletin. See following page. 


\section{HOW TO KEEP ABREAST OF NBS ACTIVITIES}

Your purchase of this publication indicates an interest in the research, development, technglogy, or service activities of the National Bureau of Standards。

The best source of current awareness in your specific area, as well as in other NBS programs of possible interest, is the TECHNICAL NEWS BULLETIN, a monthly magazine designed for engineers, chemists, physicists, research and product development managers, librarians, and company executives.

If you do not now receive the TECHNICAL NEWS BULLETIN and would like to subscribe, and/or to review some recent issues, please fill out and return the form below.

Mail to: Office of Technical Information and Publications National Bureau of Standards

Washington, D. C. 20234

Name

Affiliation

Address

City State Zip

Please send complimentary past issues of the Technical News Bulletin.

Please enter my 1-yr subscription. Enclosed is my check or money order for $\$ 3.00$ (additional $\$ 1.00$ for foreign mailing). check is made payable to: SUPERINTENDENT OF DOCUMENTS. Spec. Publ. 341 



\section{NBS TECHNICAL PUBLICATIONS}

\section{PERIODICALS}

JOURNAL OF RESEARCH reports National Bureau of Standards research and development in physics, mathematics, chemistry, and engineering. Comprehensive scientific papers give complete details of the work, including laboratory data, experimental procedures, and theoretical and mathematical analyses. Illustrated with photographs, drawings, and charts.

Published in three sections, available separately:

\section{Physics and Chemistry}

Papers of interest primarily to scientists working in these fields. This section covers a broad range of physical and chemical research, with major emphasis on standards of physical measurement, fundamental constants, and properties of matter. Issued six times a year. Annual subscription: Domestic, $\$ 9.50$; foreign, $\$ 11.75^{*}$.

\section{- Mathematical Sciences}

Studies and compilations designed mainly for the mathematician and theoretical physicist. Topics in mathematical statistics, theory of experiment design, numerical analysis, theoretical physics and chemistry, logical design and programming of computers and computer systems. Short numerical tables. Issued quarterly. Annual subscription: Domestic, $\$ 5.00$; foreign, $\$ 6.25 *$.

\section{- Engineering and Instrumentation}

Reporting results of interest chiefly to the engineer and the applied scientist. This section includes many of the new developments in instrumentation resulting from the Bureau's work in physical measurement, data processing, and development of test methods. It will also cover some of the work in acoustics, applied mechanics, building research, and cryogenic engineering. Issued quarterly. Annual subscription: Domestic, $\$ 5.00$; foreign, $\$ 6.25 *$.

\section{TECHNICAL NEWS BULLETIN}

The best single source of information concerning the Bureau's research, developmental, cooperative and publication activities, this monthly publication is designed for the industry-oriented individual whose daily work involves intimate contact with science and technology—for engineers, chemists, physicists, research managers, product-development managers, and company executives. Annual subscription: Domestic, $\$ 3.00$; foreign, $\$ 4.00^{*}$.

\section{NONPERIODICALS}

Applied Mathematics Series. Mathematical tables, manuals, and studies.

Building Science Series. Research results, test methods, and performance criteria of building materials, components, systems, and structures.

Handbooks. Recommended codes of engineering and industrial practice (including safety codes) developed in cooperation with interested industries, professional organizations, and regulatory bodies.

Special Publications. Proceedings of NBS conferences, bibliographies, annual reports, wall charts, pamphlets, etc.

Monographs. Major contributions to the technical literature on various subjects related to the Bureau's scientific and technical activities.

National Standard Reference Data Series. NSRDS provides quantitative data on the physical and chemical properties of materials, compiled from the world's literature and critically evaluated.

Product Standards. Provide requirements for sizes, types, quality and methods for testing various industrial products. These standards are developed cooperatively with interested Government and industry groups and provide the basis for common understanding of product characteristics for both buyers and sellers. Their use is voluntary.

Technical Notes. This series consists of communications and reports (covering both other agency and NBS-sponsored work) of limited or transitory interest.

Federal Information Processing Standards Publications. This series is the official publication within the Federal Government for information on standards adopted and promulgated under the Public Law 89-306, and Bureau of the Budget Circular A-86 entitled, Standardization of Data Elements and Codes in Data Systems.

- Difference in price is due to extra cost of foreign mailing.

Order NBS publications from:

Superintendent of Documents

Government Printing Office

Washington, D.C. 20402 
U,S. DEPARTMENT OF COMMERCE

WASHINGTON, D.C. 20230

OFFICIAL BUSINESS

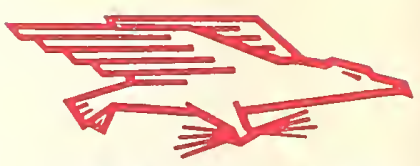

POSTAGE AND FEES PAID

U.8. DEPARTMENT OF COMMERCE

PENALTY FOR PRIVATE USE, $\$ 300$ 